\title{
Hanford Site Annual Dangerous Waste Report
}

Volume 2

Generator Dangerous Waste Report

Radioactive Mixed Waste

Calendar Year 1994

United States

Department of Energy

Richland, Washington 99352

\section{DISCLAIMER}

This report was prepared as an account of work sponsored by an agency of the United States Government. Neither the United States Government nor any agency thereof, nor any of their employees, makes any warranty, express or implied, or assumes any legal liability or responsibility for the accuracy, completeness, or usefulness of any information, apparatus, product, or process disclosed, or represents that its use would not infringe privately owned rights. Reference herein to any specific commercial product, process, or service by trade name, trademark, manufacturer, or otherwise does not necessarily constitute or imply its endorsement, recommendation, or favoring by the United States Government or any agency thereof. The views and opinions of authors expressed herein do not necessarily state or reflect those of the United States Government or any agency thereof.

MASTER 


\section{DISCLAIMER}

Portions of this document may be illegible in electronic image products. Images are produced from the best available original document. 
OTE: DEE'16 DPR

ATTI OF: EAP:EBD 93-RPA-071

SESAET: DELEGATION OF SIGRATURE AUTHORITY FOR THE ANNUAL DANGEROUS WASTE REPORT

$+\infty$

To: James D. Bauer, Acting Program Manager

Office of Environmental Assurance,

Permits, and Policy

'I hereby delegate signature authority for the Annual Dangerous Yaste Repor $\dot{t}$ to the Program Manager, Office of Environmental Assurance, Permits, and Policy.

Pursuant to the Hazardous Waste Reporting.Forms Packet. for Calendar Year 1991, Part I, Form 4, Generator Annual Dangerous Waste Report, Section 11, signature authority may be delegated to the manager's authorized representative. This memorandum constitutes formal delegation of such authority to the Program Hanager, Office of Environmental Assurance, Permits, and Policy.

Pursuant to the Hazardous Waste Reporting Forms Packet for Calendar Year 1991, Part III, Form 5, Vaste Management Facility Annual Dangerous Waste Report, Section 12, signature authority may be delegated to the manager's authorized representative. This memorandum constitutes formal delegation of such authority to the Program Manager, Office of Environmental Assurance, Peraits, and Policy. 
Form 41994 GENERATOR ANNUAL DANGEROUS WASTE REPORT

PLEASE PRINT OR TYPE - Blue or Black ink Only - (Form designed for use on Ellte (12 pltch) typewrilter) Use spacebar between each character

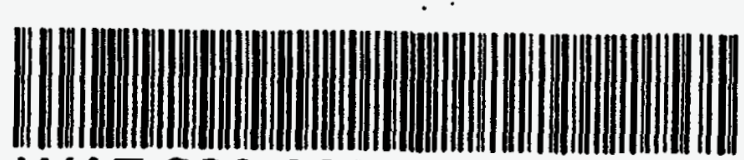

WA7 890008967 A F5 94 DEPARTMENT OF ENERGY

PO BOX 550

RICHLAND

WA 99352 1.
SPAISTATE HAZARDOUS WASTE
SITENTIFICATION NUMBER$$
2 .
$$

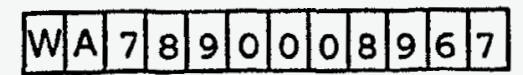

2. COMPANY NAME Soe Page 5

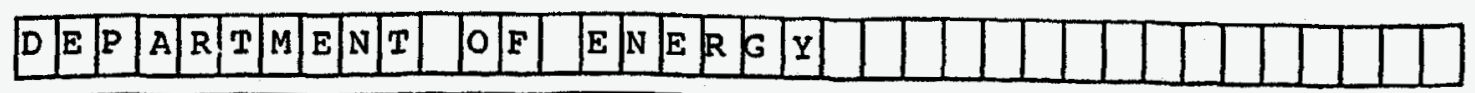

3. SITE CONTACT PERSON, AND TITLE PHONE NUMBER

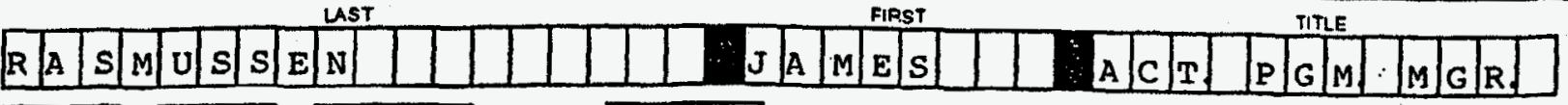

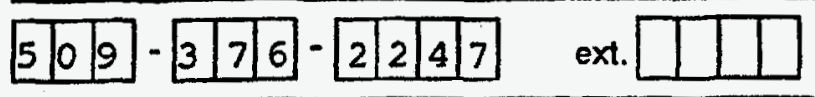

4. SITE CONTACT MAILING ADDRESS

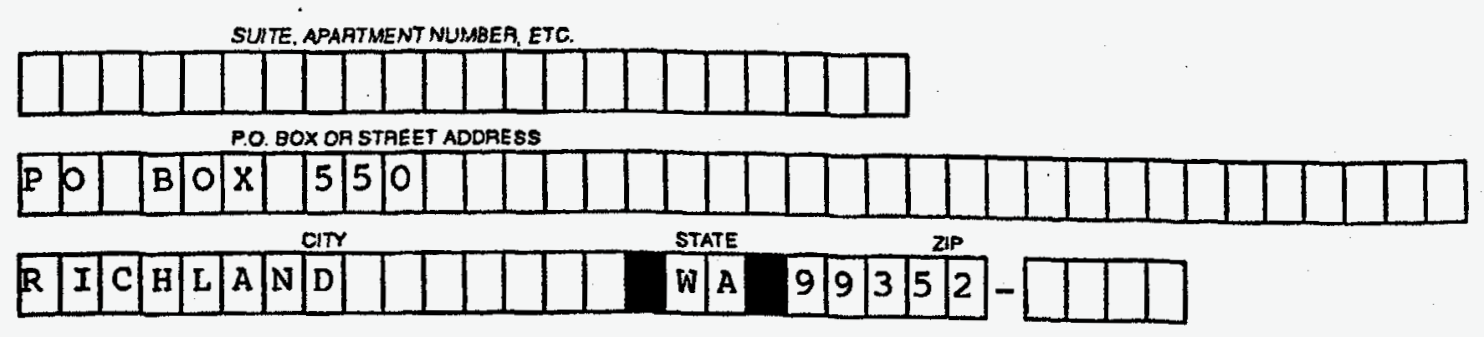

See Page 5

5. SITE LOCATION ADDRESS

See Page 5

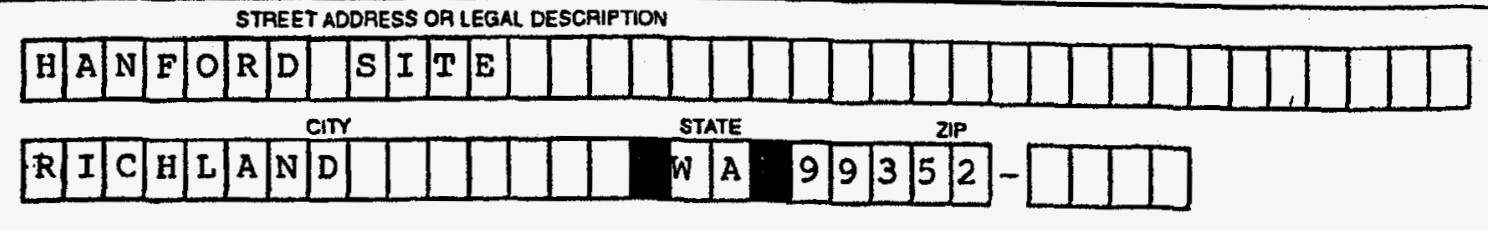

6. SITE LOCATION COUNTY

See Page 5

DUE DATE: Postmanked

No Later Than

MARCH 1, 1995

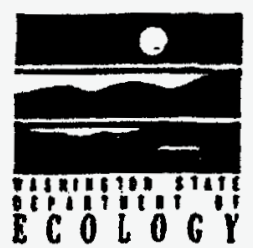

7. WASHINGTON DEPT. OF REVENUE SALES TAX REGISTRATION NUMBER (UBI NUMBER)

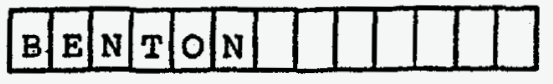

See Page 6

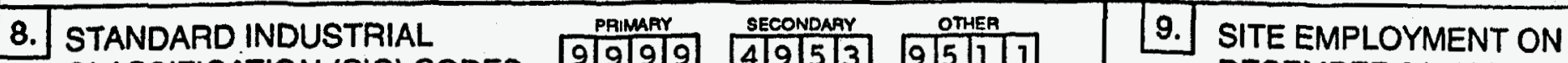

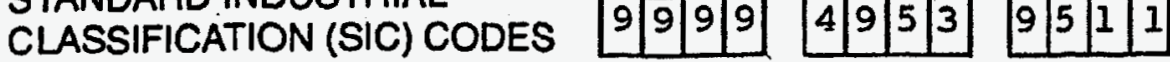
See Page 0 DECEMBER 31, 1994

E.

11. CERTIFICATION - I certiy thal under penalty of law that I have personally examined and am faniliar with the information submitted in this and all attached documents, and that based on my inquiry of tho Selieve that the submithed informalion is true, accurate, and complele. I am aware
James E. Rasmussen, Acting Program Manager James E. Rasmussen, Acting Program Manager Wamer 2 Ramasenas $2 / 22 / 95$ Page 1 of 161 Pages ; ECY $28 B$ (09/94) Form A Frme 


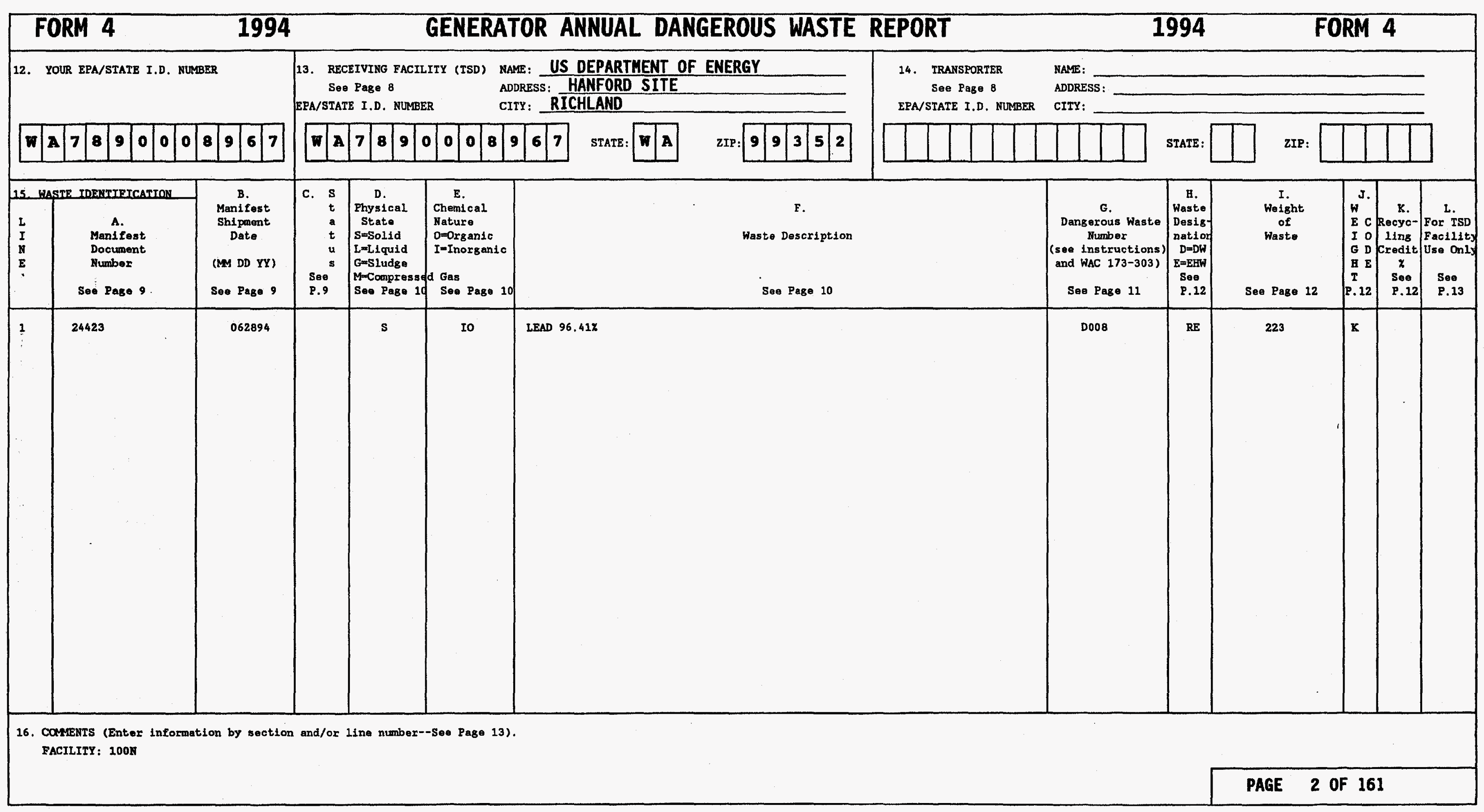




\begin{tabular}{|c|c|c|c|c|c|c|c|c|c|c|c|c|c|}
\hline & DRM 4 & 199 & & & GENERAT & OR ANNUAL DANGEROUS WASTE & REPORT & & 1994 & & $\overline{\mathrm{DMM}}$ & & \\
\hline & OUR EPA/STATE I.D. & & $\begin{aligned} & \text { 13. } \mathrm{RE} \\
& \mathrm{Se} \\
& \mathrm{SPA} / \mathrm{STA}\end{aligned}$ & $\begin{array}{l}\text { CEIVING FACII } \\
\text { A Page } 8 \\
\text { TE I.D. NUMBE }\end{array}$ & $\begin{array}{ll}\text { LITY (TSD) } & \text { NAM } \\
\text { ER } & \text { ADD } \\
\text { CII }\end{array}$ & $\begin{array}{l}\text { II: US DEPARTMENT OF ENERGY } \\
\text { IRES: } \frac{\text { HANFORD SITE }}{\text { RICHLAND }} \\
\end{array}$ & $\begin{array}{l}\text { 14. TRANSPORTER } \\
\text { Sea Page } 8 \\
\text { EPA/STAIE I.D. NUI }\end{array}$ & $\begin{array}{l}\text { NAME: } \\
\text { ADDRESS: } \\
\text { CITY: }\end{array}$ & & & & & \\
\hline$\checkmark$ & \begin{tabular}{llll|l|l} 
& 7 & 8 & 9 & 0 & 0
\end{tabular} & \begin{tabular}{l|l|l|l}
8 & 9 & 6 & 7
\end{tabular} & wh & \begin{tabular}{l|l|l|l}
7 & 8 & 9 & 1 \\
\end{tabular} & \begin{tabular}{l|l|l|l|l}
0 & 0 & 0 & 8 & 9
\end{tabular} & 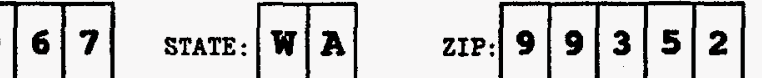 & & & STATE: & zIP: & & & \\
\hline & STE IDENTIFICATTON & & c. $s$ & & & & & & н. & I. & J: & & \\
\hline \begin{tabular}{|l}
$L$ \\
$I$ \\
$\mathbb{N}$
\end{tabular} & $\begin{array}{l}\text { A. } \\
\text { Manifest } \\
\text { Document } \\
\text { Number } \\
\text { Soe Pago } 9\end{array}$ & 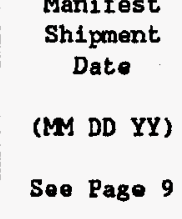 & $\begin{array}{r}t \\
t \\
u \\
\text { sea } \\
\text { P.9 }\end{array}$ & $\begin{array}{l}\text { Paysical } \\
\text { State } \\
\text { S=Solid } \\
\text { L=Liquid } \\
\text { G=Sludge } \\
\text { M-Compress } \\
\text { Seo Page 10 }\end{array}$ & $\begin{array}{l}\text { Nature } \\
\text { Naturg } \\
\text { O=Organic } \\
\text { I=Inorganic } \\
\text { d Gas } \\
\text { Seo Page } 10\end{array}$ & $\begin{array}{l}\text { Waste Description } \\
\text { See Page } 10\end{array}$ & & $\begin{array}{c}\text { Dangerous Waste } \\
\text { Number } \\
\text { (see instructions) } \\
\text { and WAC 173-303) } \\
\text { See Page } 11\end{array}$ & 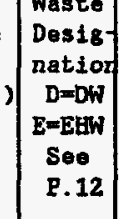 & See Page 12 & 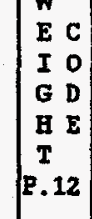 & $\begin{array}{c}\text { Recyc- } \\
\text { 1ng } \\
\text { credit } \\
x \\
\text { Soe } \\
\text { P.12 }\end{array}$ & \begin{tabular}{|l|} 
For TsD \\
Facility \\
Use Only \\
So: \\
P.13
\end{tabular} \\
\hline 1 & 24177 & 061394 & & $\mathrm{~s}$ & Io & $\begin{array}{l}1,1,1 \text { TRICHLORETHANE } 2.73 \% \text {, BUTANE .03\%, DTOXOLANE } \\
.3 \mathrm{y}\end{array}$ & B25\%。 PHOSRHORIC ACID & $\begin{array}{l}\text { F002 WP01 } \\
\text { WT02 }\end{array}$ & $\mathrm{RE}$ & 4 & $\mathrm{k}$ & & \\
\hline 2 & 24515 & 090994 & & L & 。 & LABPACK OF PAINTS & & $\begin{array}{l}\text { D001 D007 } \\
\text { D008 D018 } \\
\text { D019 D035 } \\
\text { WL01 hC02 } \\
\text { WP01 WT01 }\end{array}$ & $\mathrm{RE}$ & 52.1 & $\mathrm{x}$ & & \\
\hline 3 & 24515 & 090994 & & Ls & o & LABPACK OF PAINTS & & $\begin{array}{l}\text { D001 D007 } \\
\text { D008 D018 } \\
\text { D035 WL.01 } \\
\text { WCO2 WP01 } \\
\text { WT02 }\end{array}$ & $\mathrm{RE}$ & 29.9 & $\mathbf{k}$ & & $\therefore$ \\
\hline 14 & 24735 & 083194 & & $\mathrm{~L}$ & I & HYDROCHLORIC ACID 30.4 & & D002 WT02 & $\mathrm{RD}$ & 20 & $\mathbf{x}$ & & \\
\hline
\end{tabular}

16. COMMENTS (Entex information by section and/ox line number--See Page 13). FACILITY: 1058 


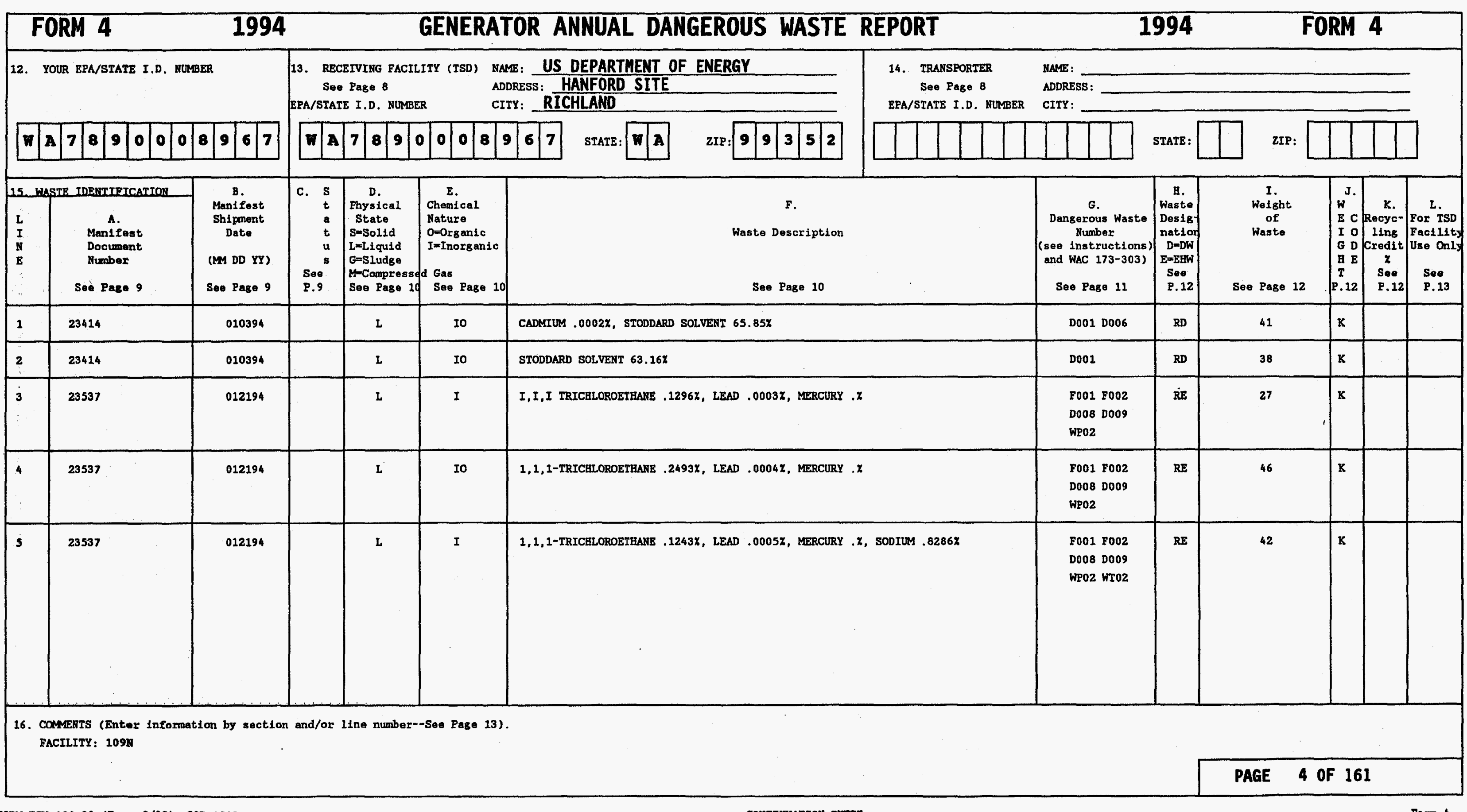




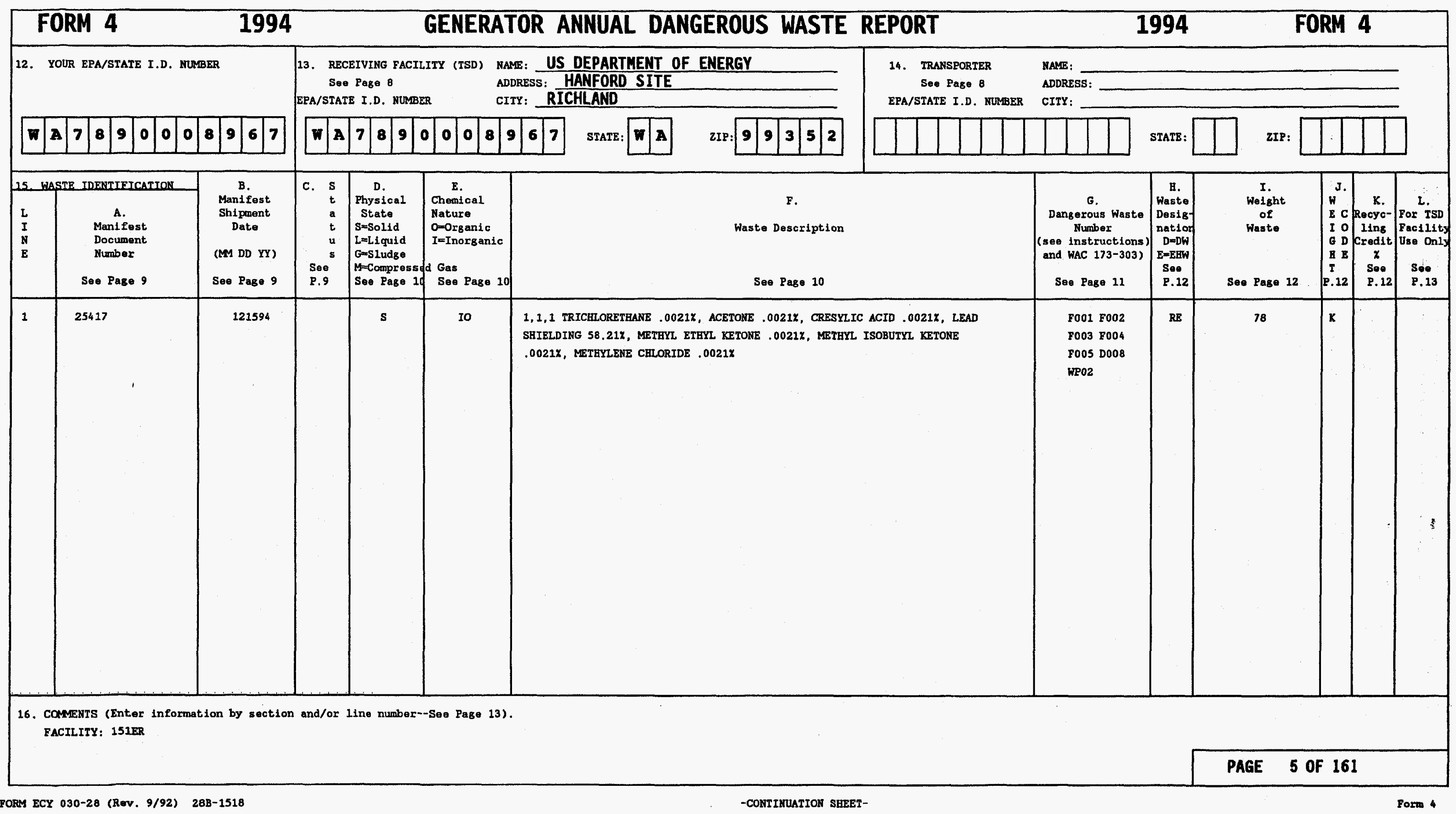




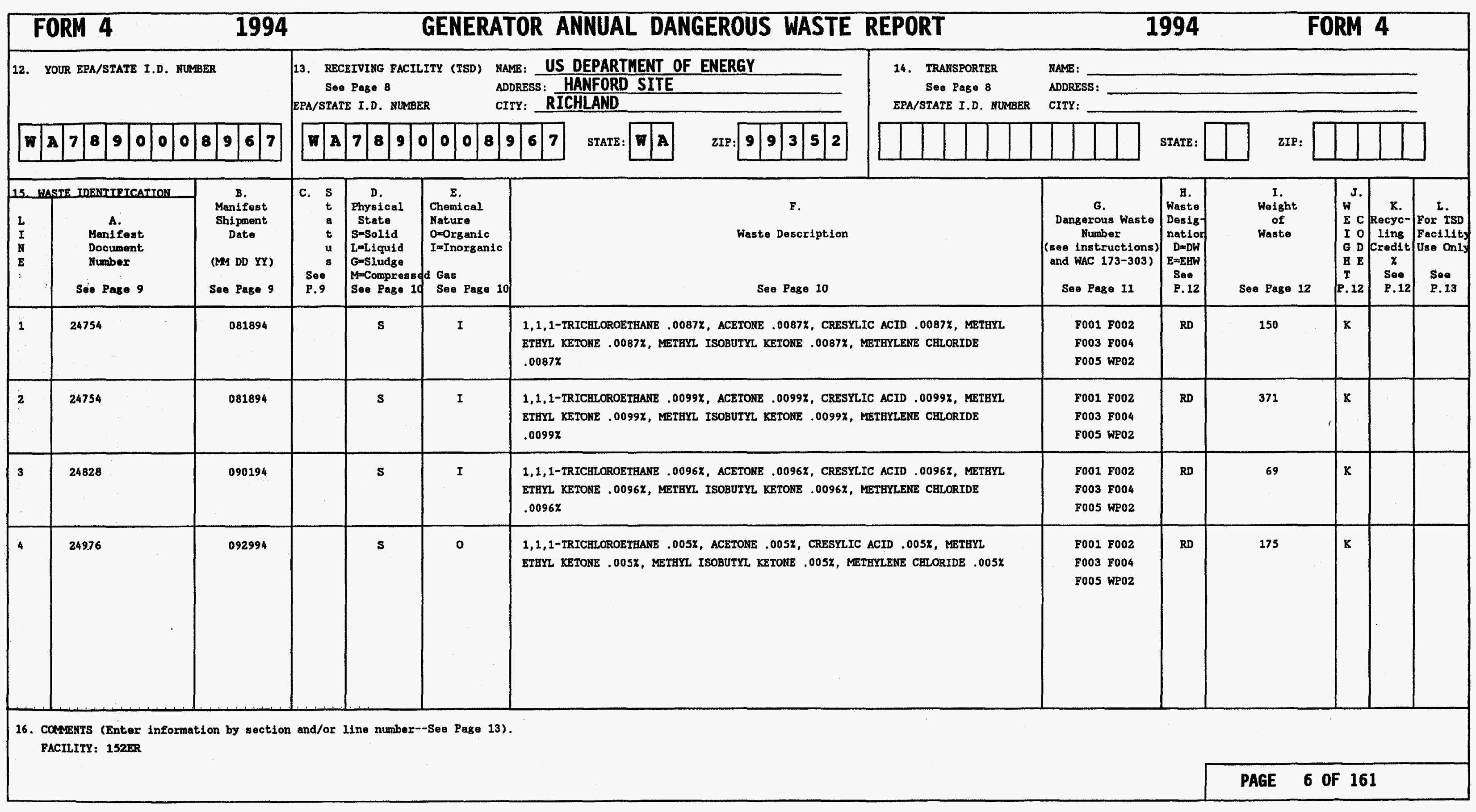




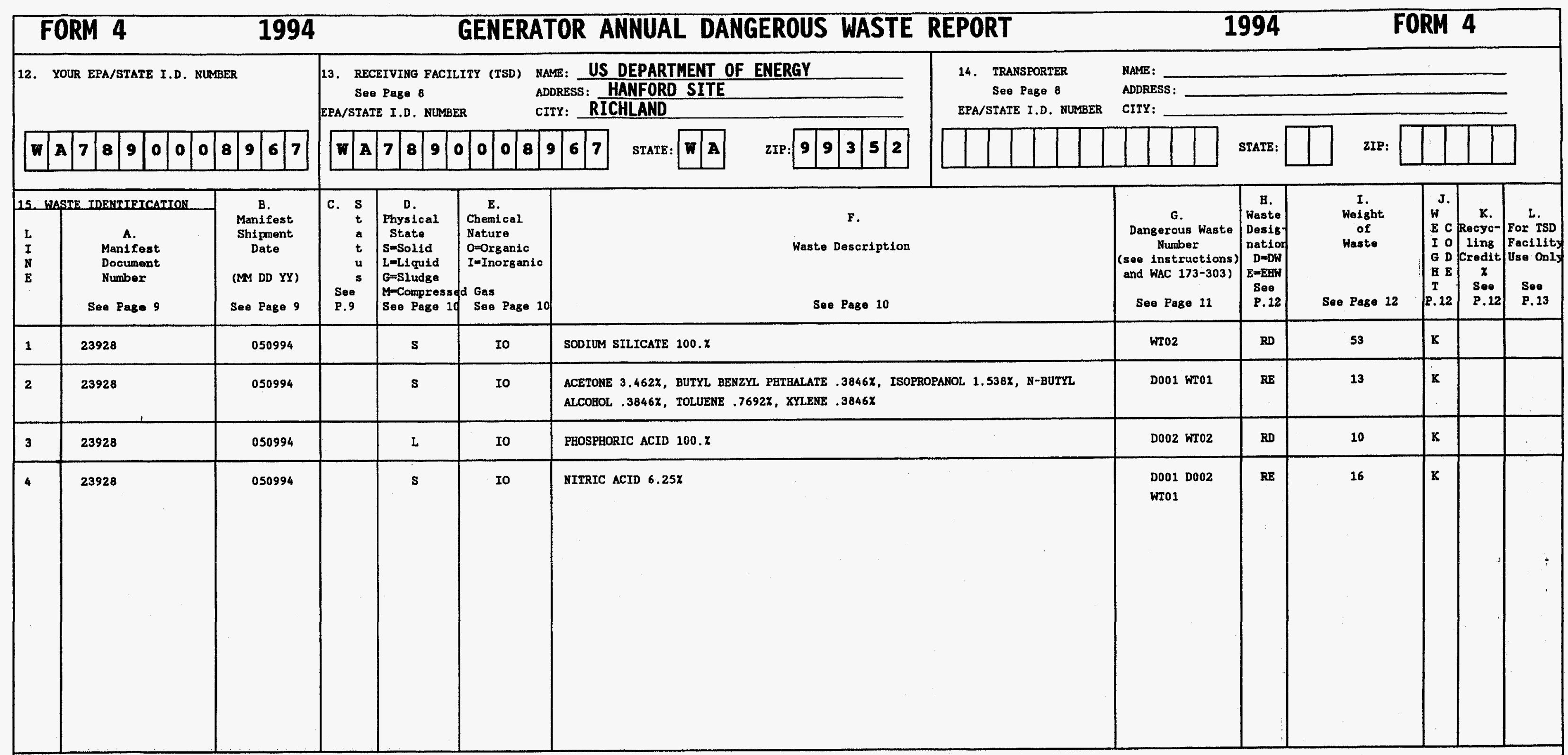

16. Comerewss (Enter information by section and/or line number--See Page 13).

FACIIITY: 1639 


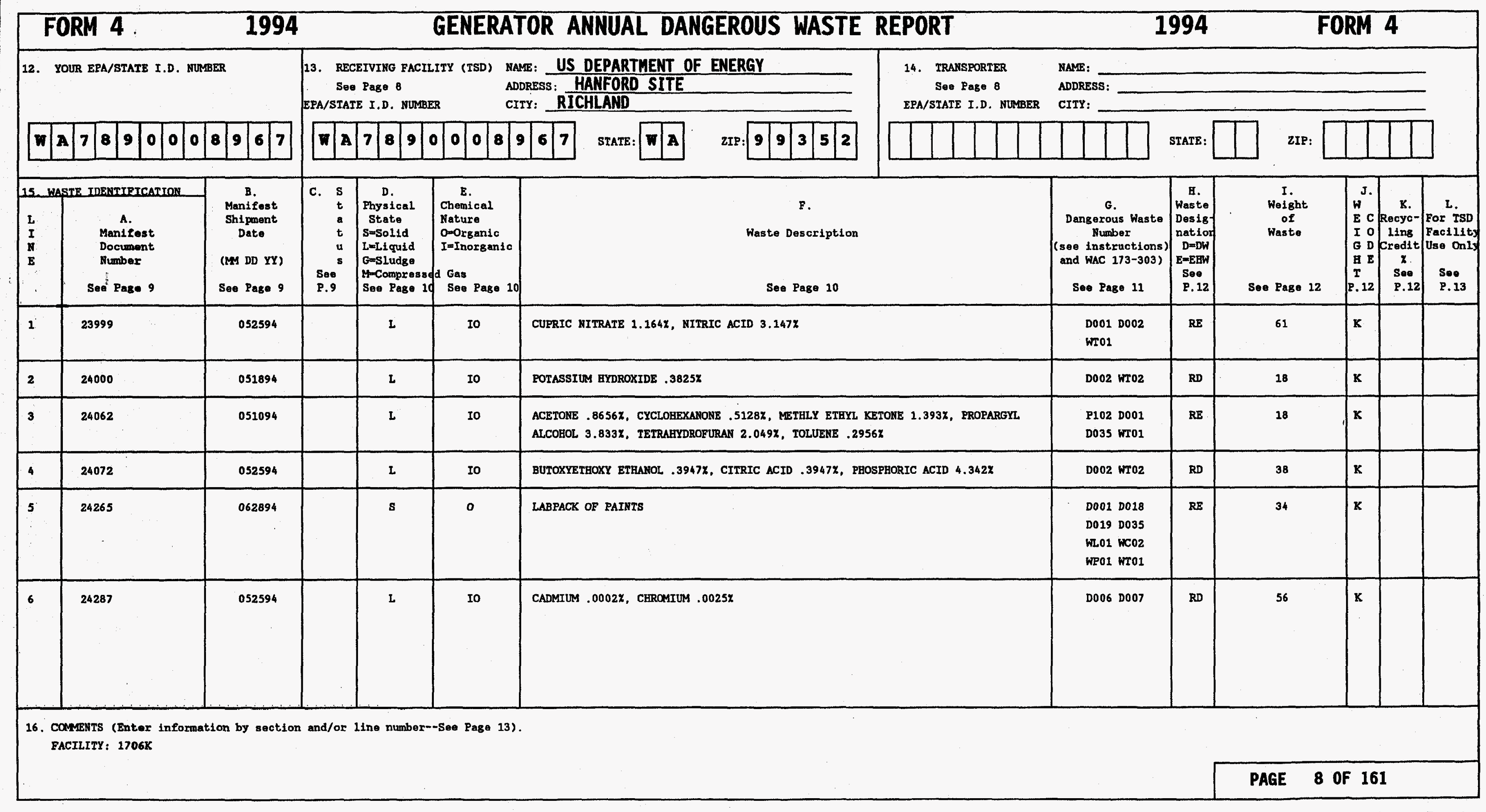




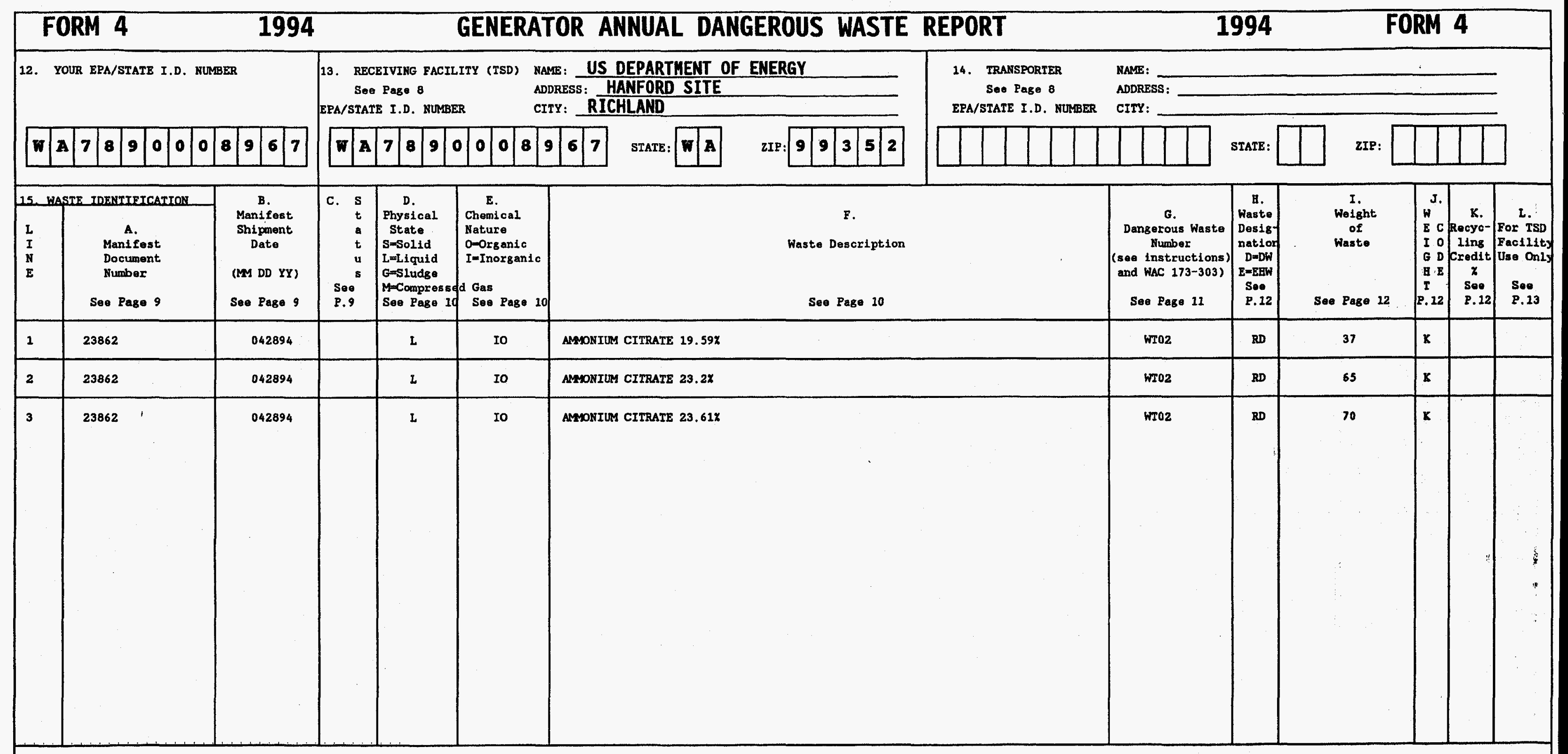

16. CaMENTS (Enter information by section and/or 1ine number--See Page 13). FACILITY: $1706 \mathrm{KE}$ 


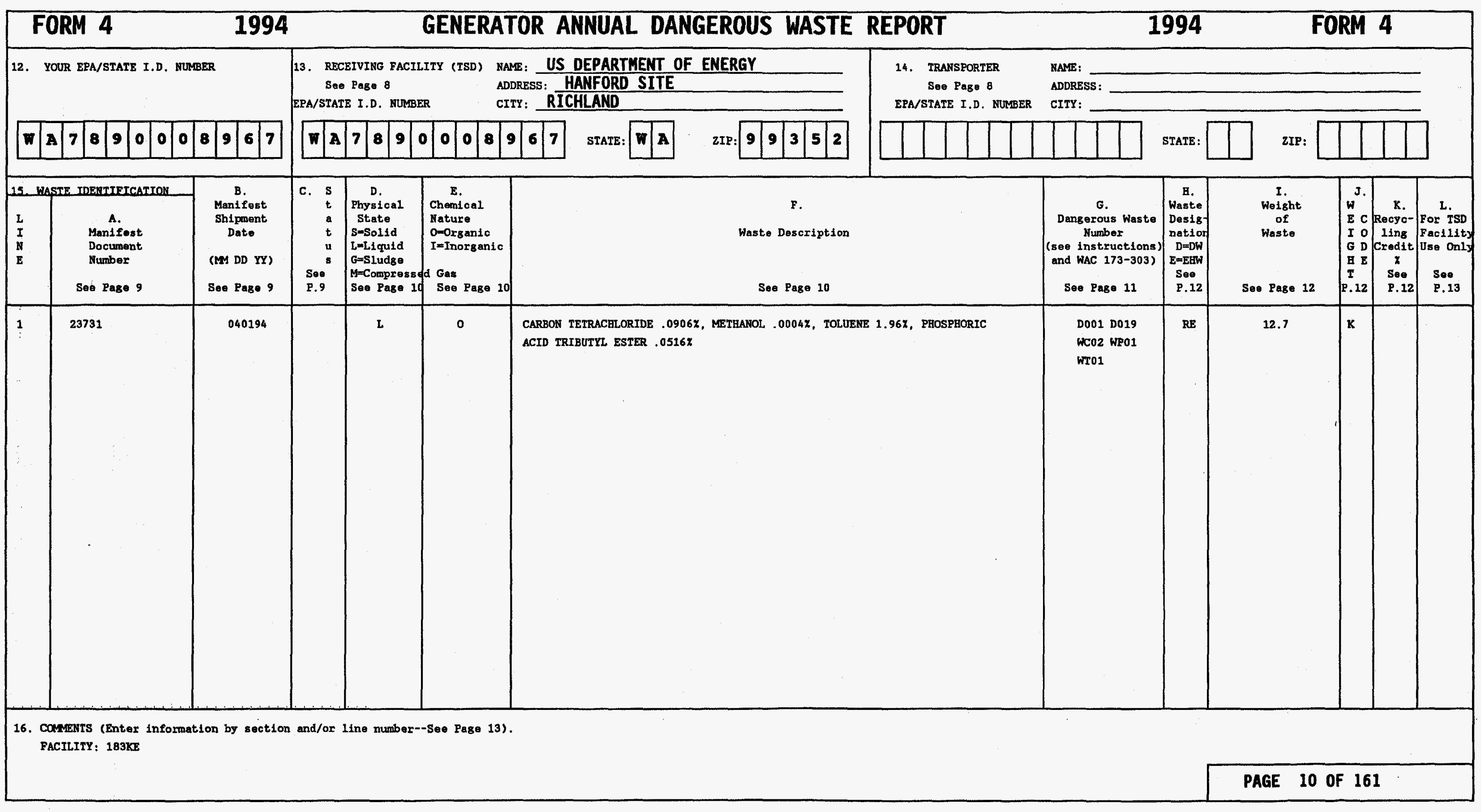




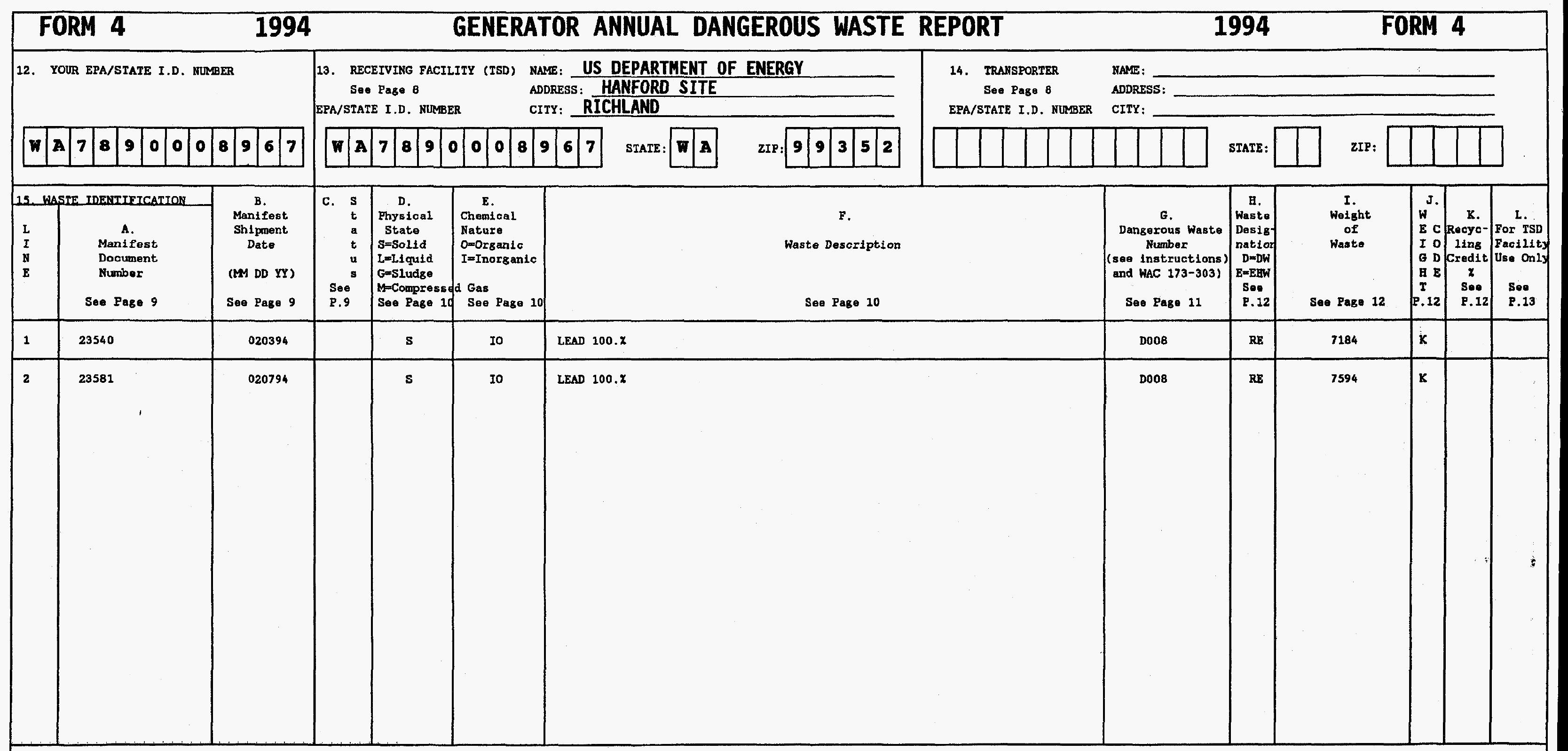

16. Comexrs (Enter information by section and/or line number--See Page 13). FACILITY: $190 \mathrm{C}$ 


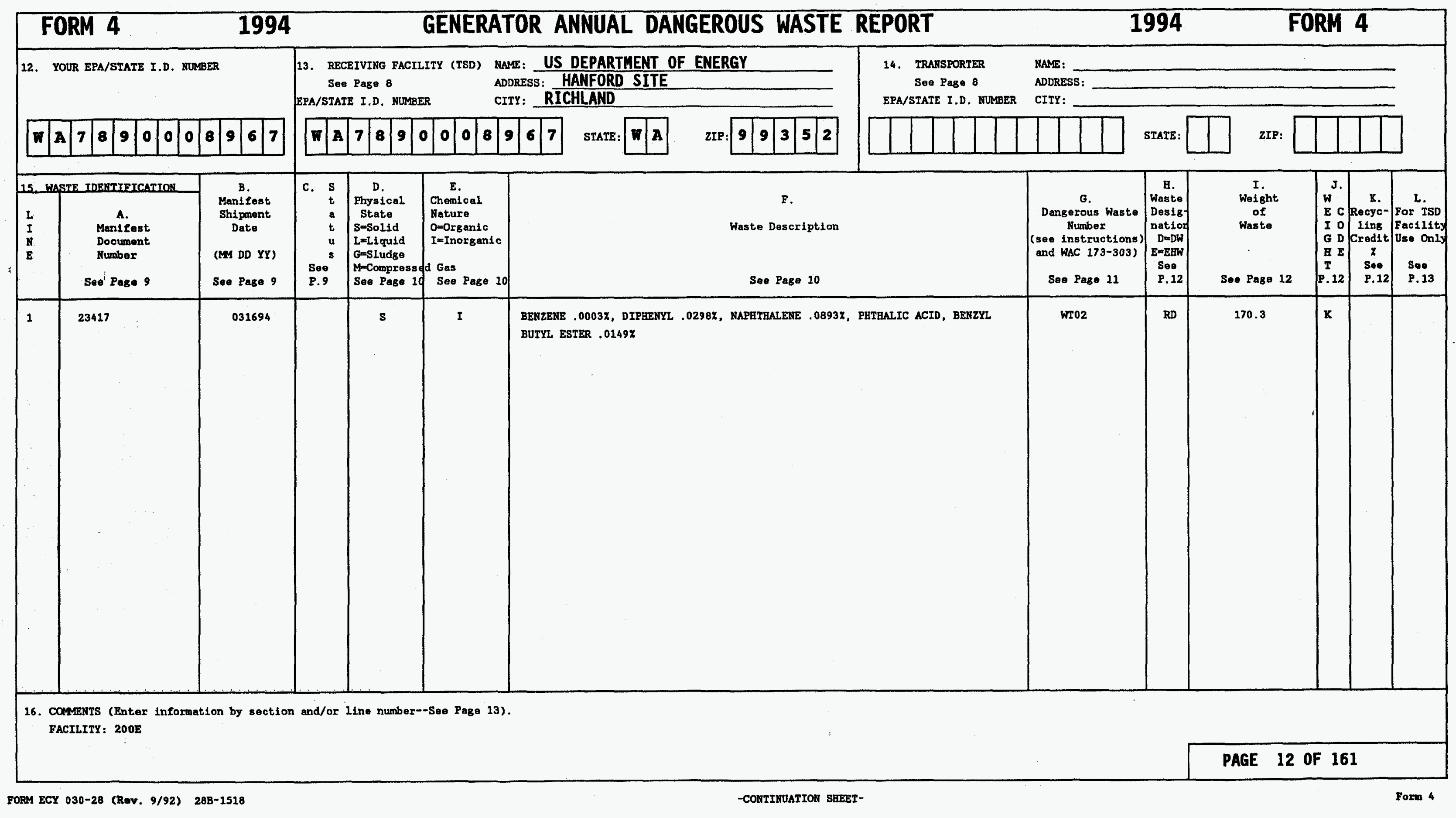




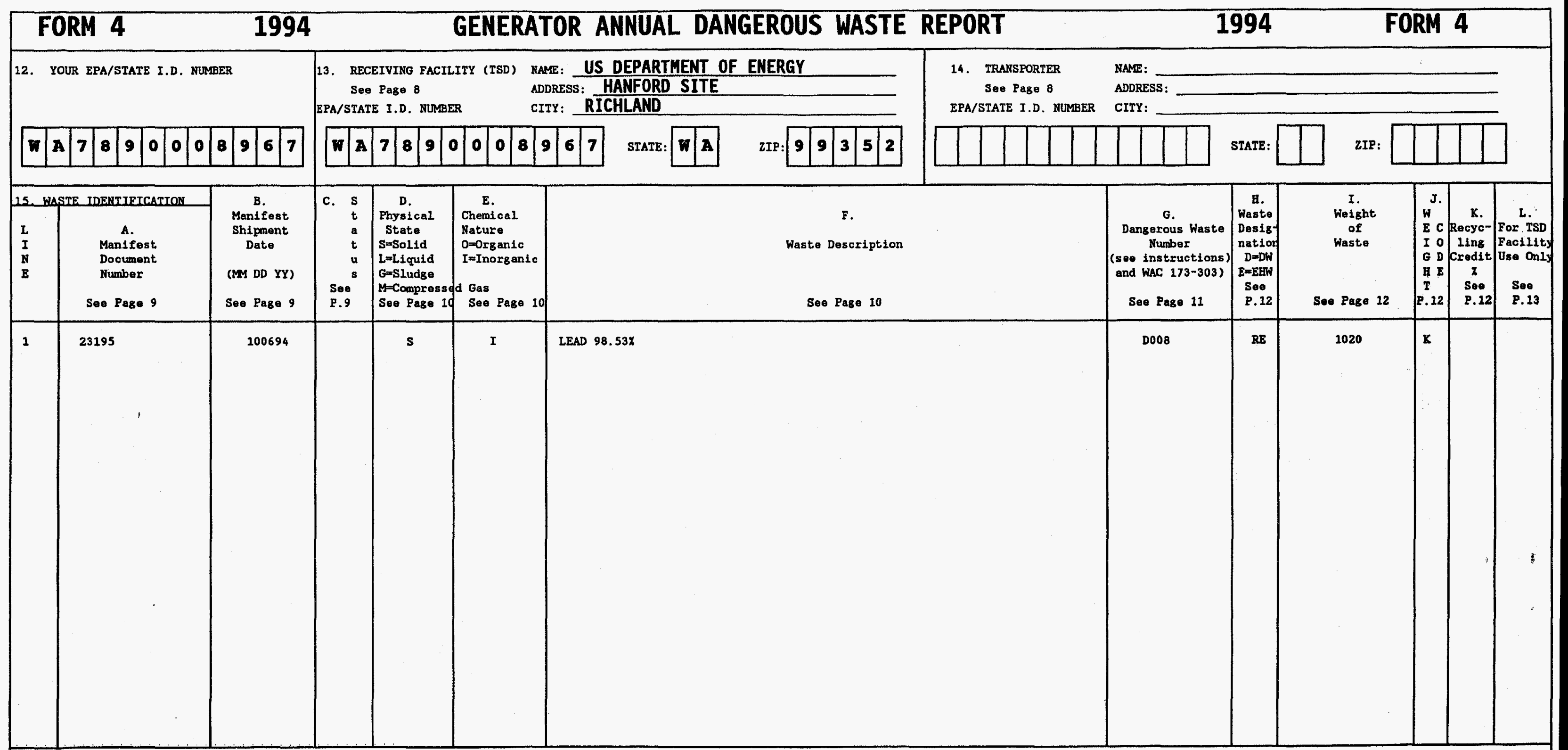

16. CoMENTS (Enter information by section and/or line number--See Page 13). FACILITY: 200W 


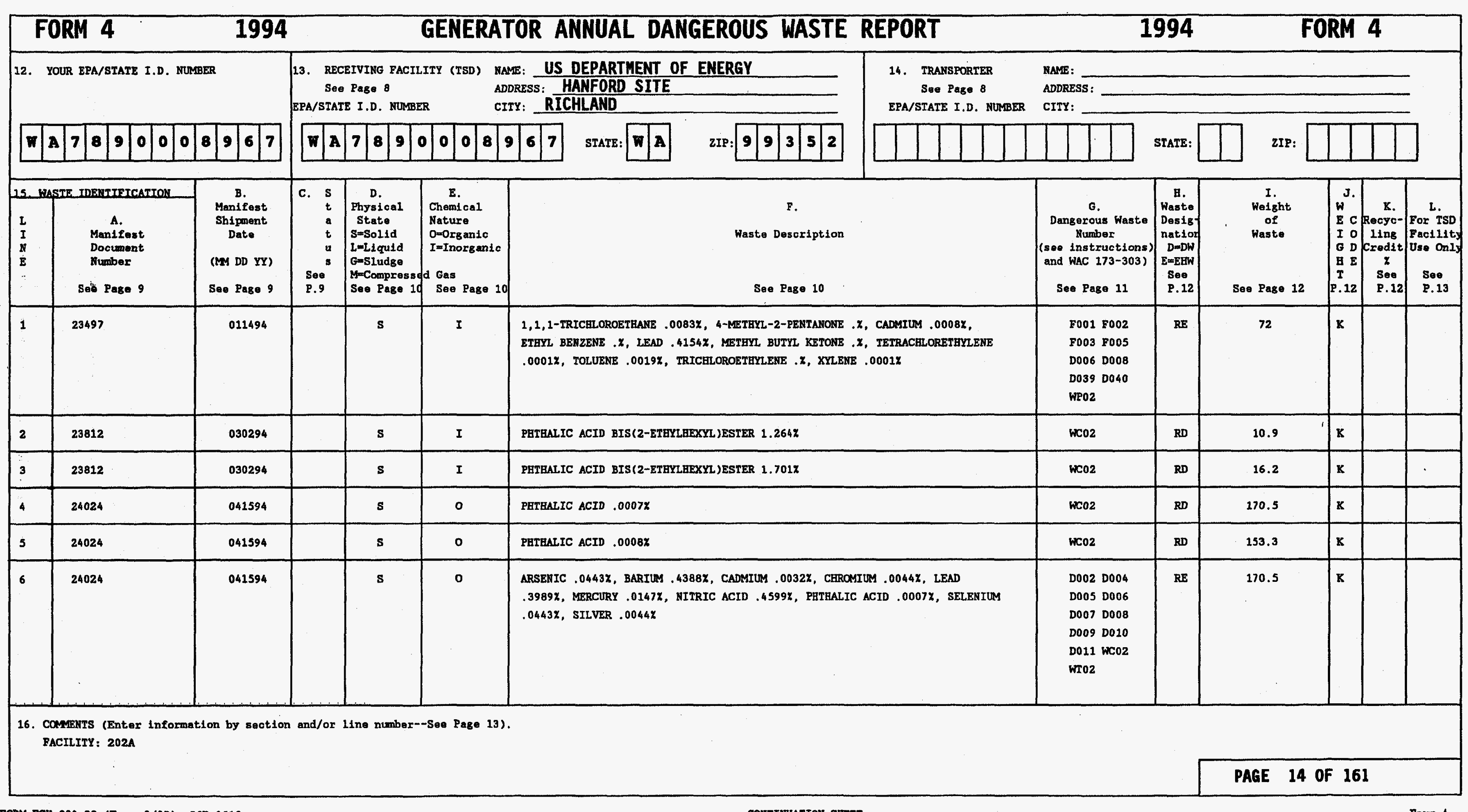




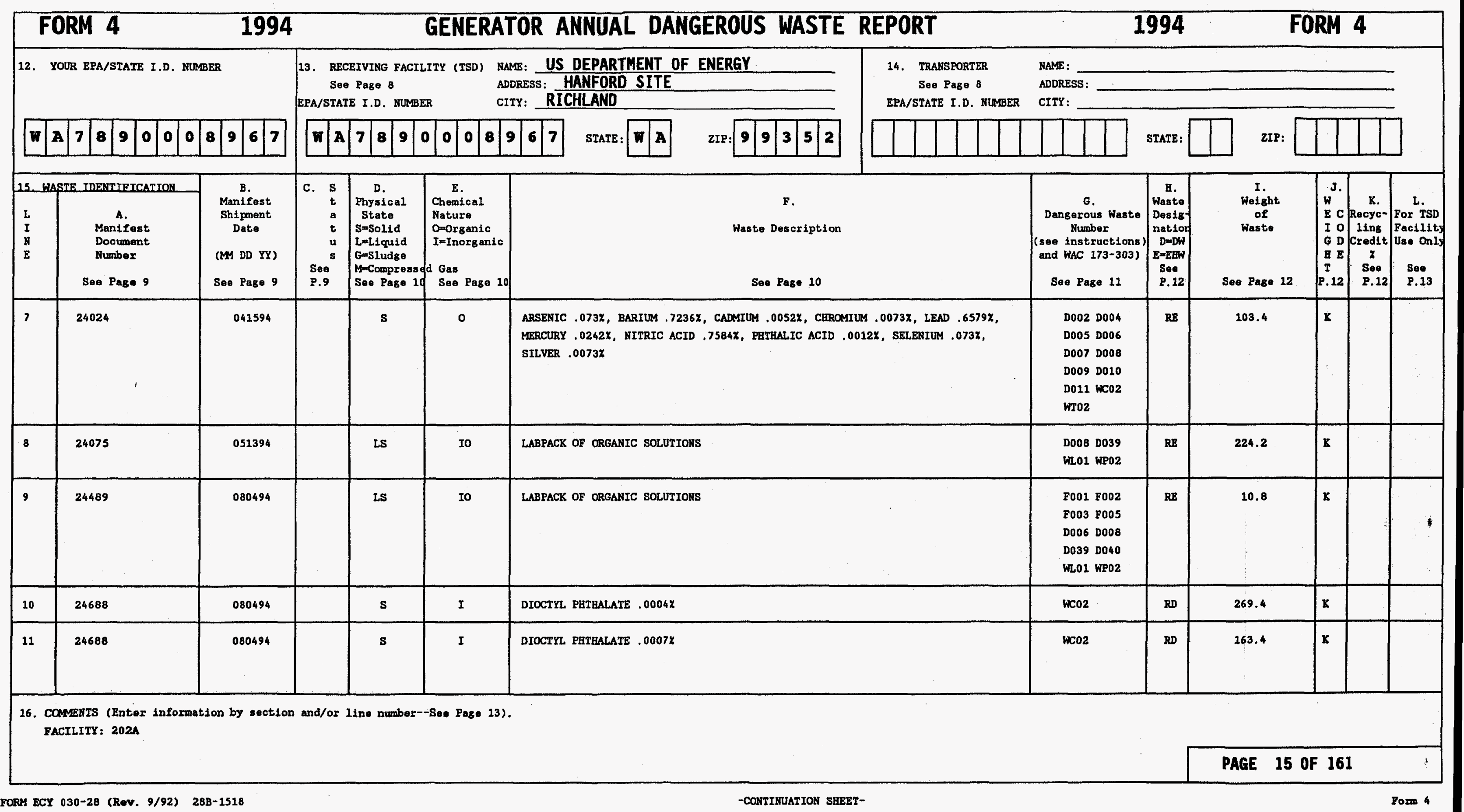




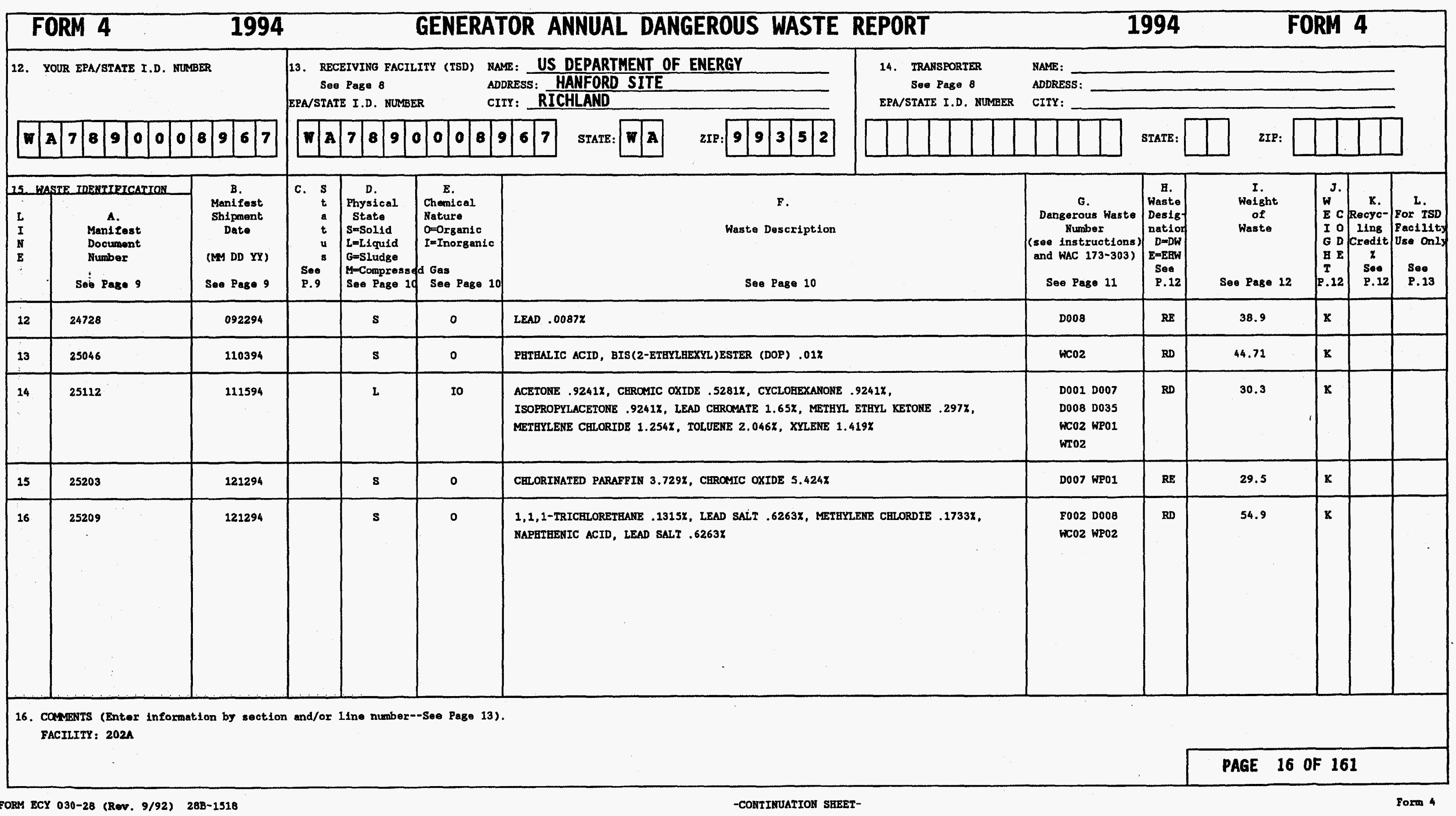




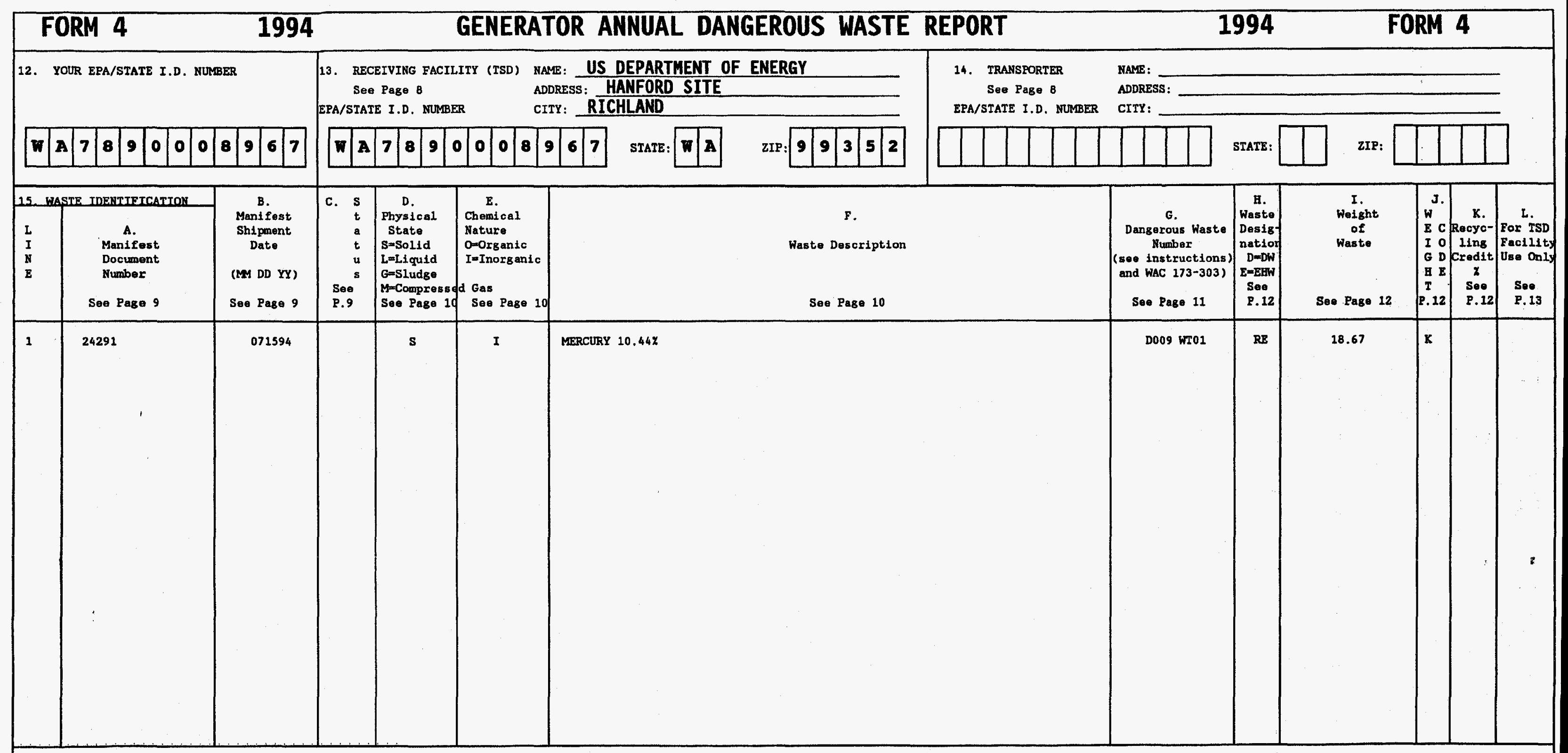

16. Comerers (Enter information by section and/or line number--Sea Page 13). FACILITY: 202AL 


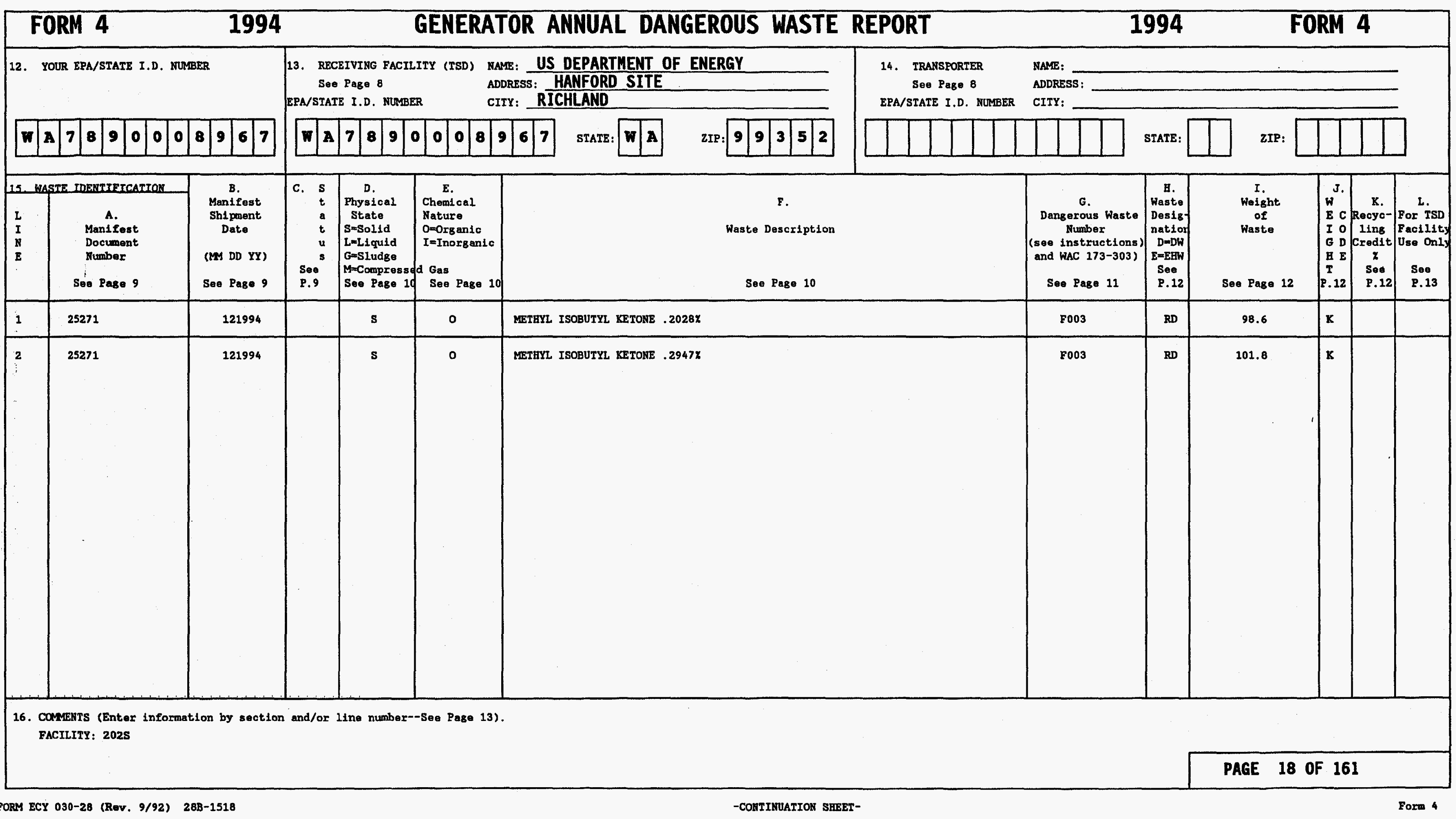




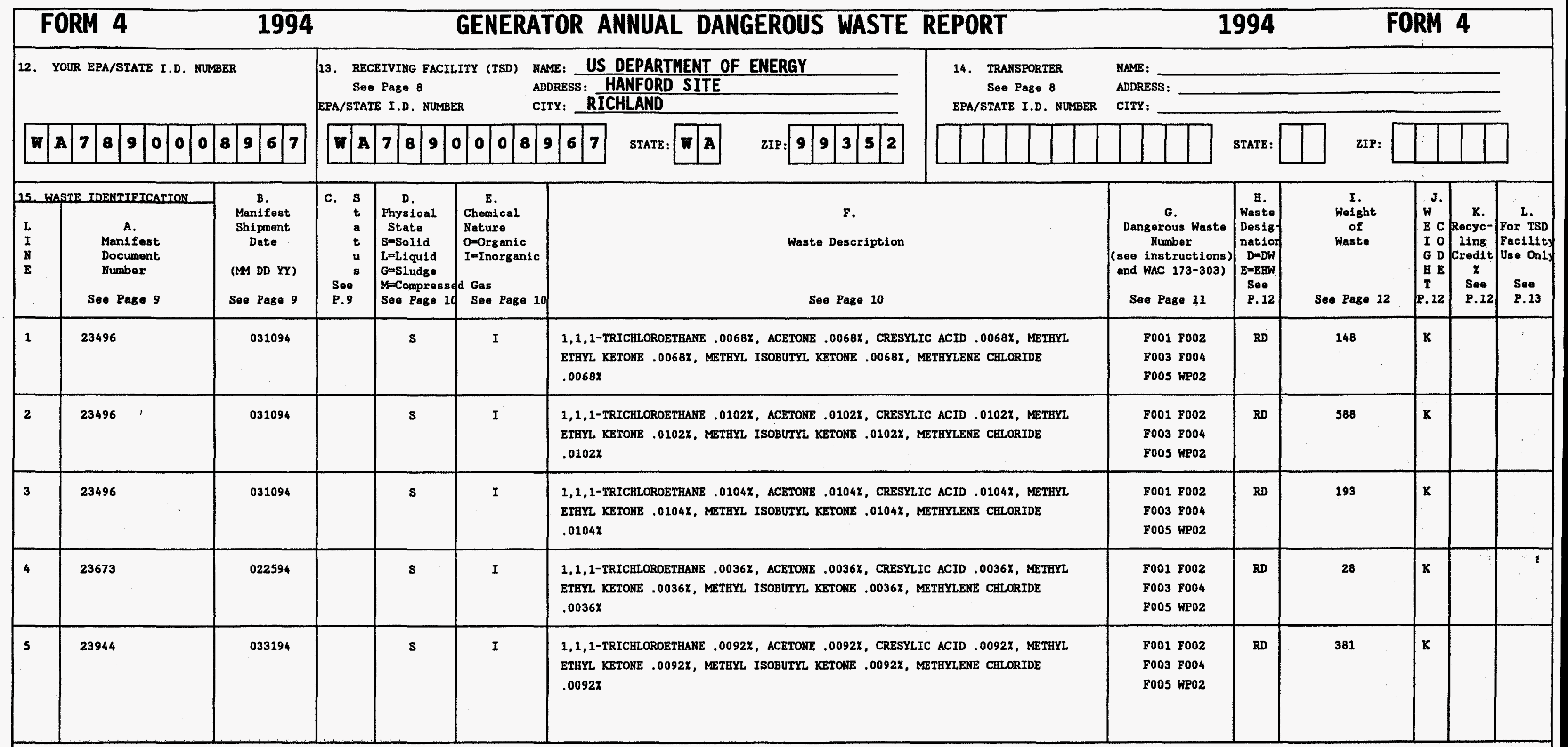

16. COMMENTS (Entor Inforwation by section and/or line number--See Page 13). FACILITY: 204AR

PAGE 19 OF 161 


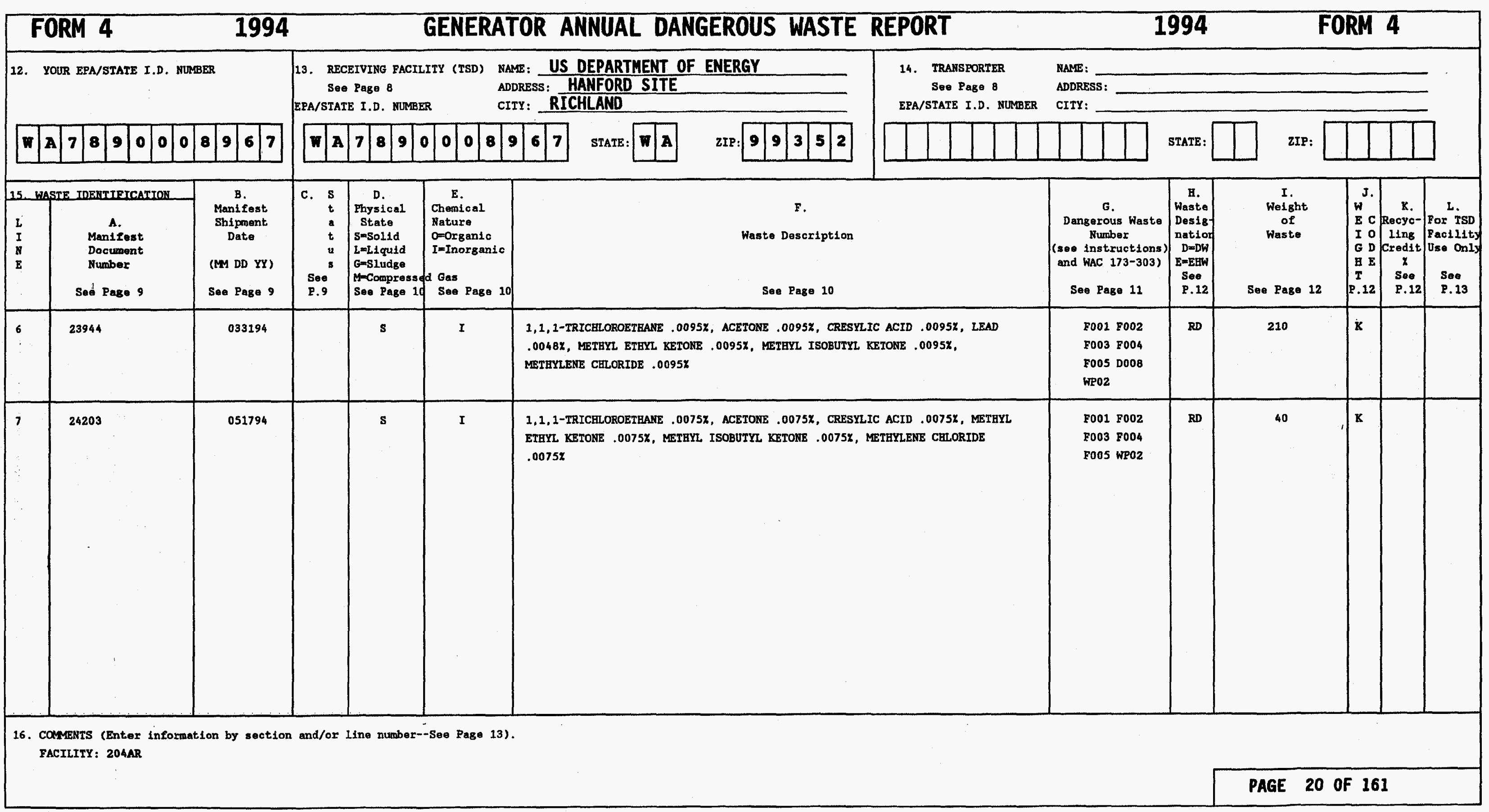




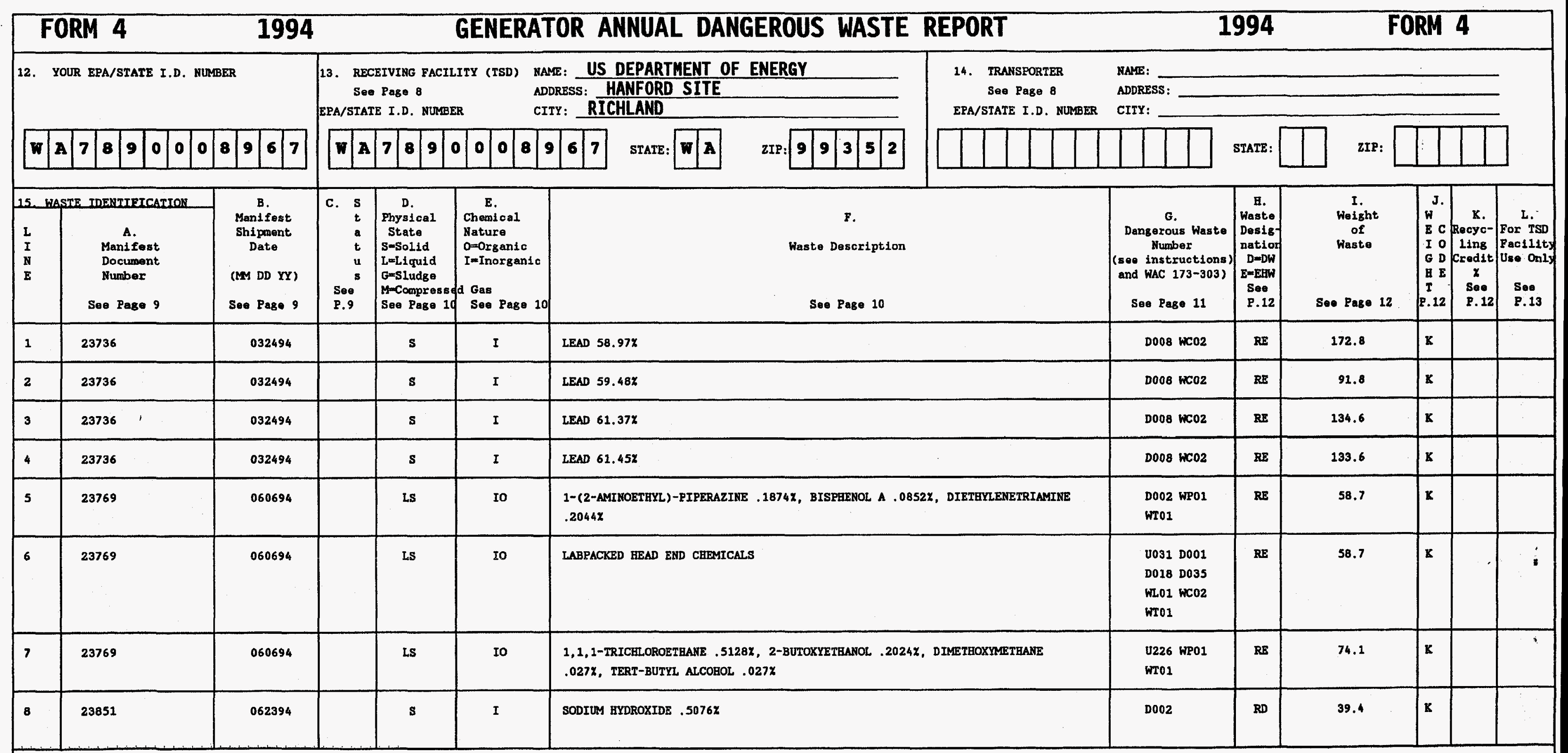

16. CaMmenrs (Enter information by section and/or line number--See Page 13). FACILITY: 221T 


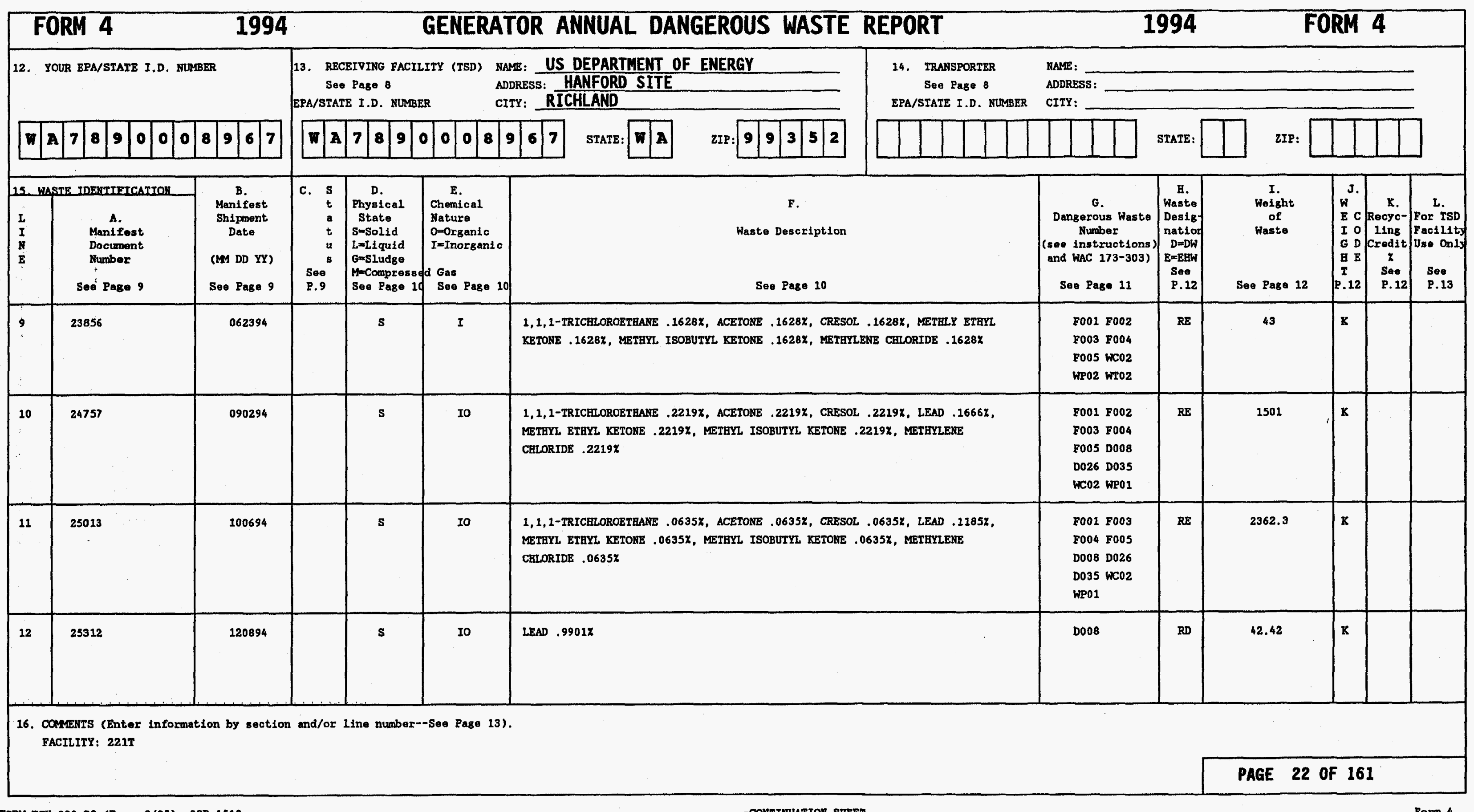




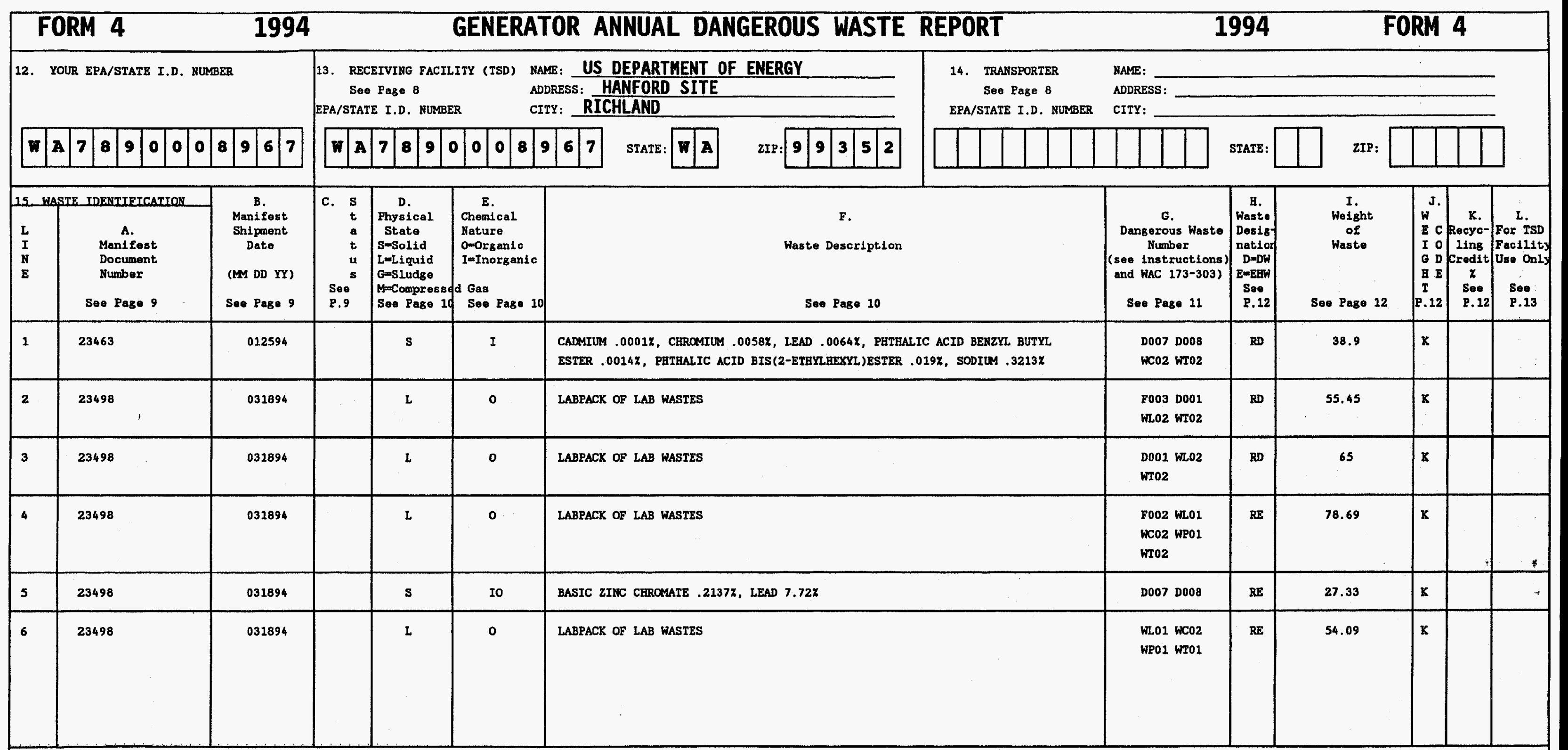

16. COMMENTS (Enter information by section and/or line number--See Page 13). FACILITY: 222S 


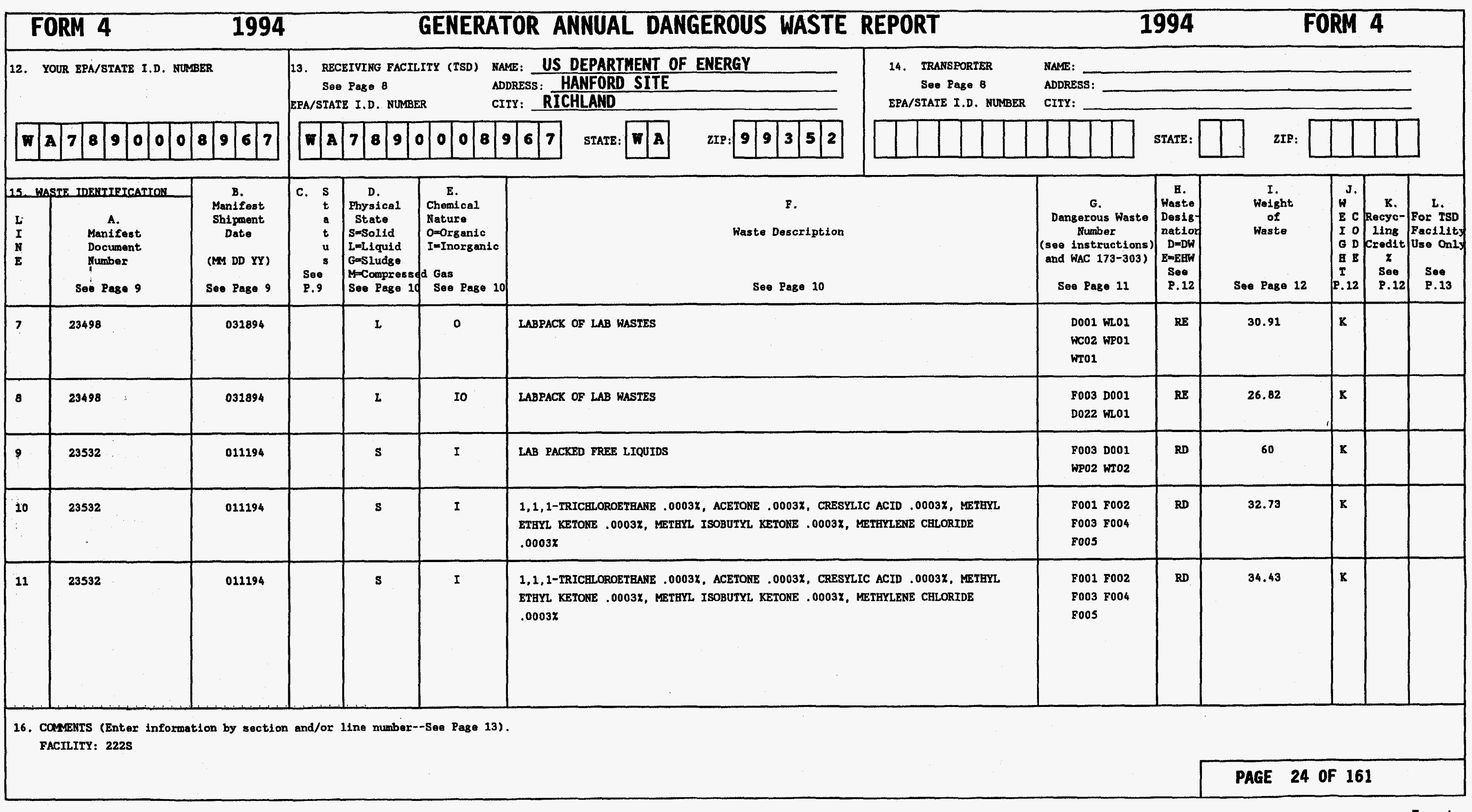




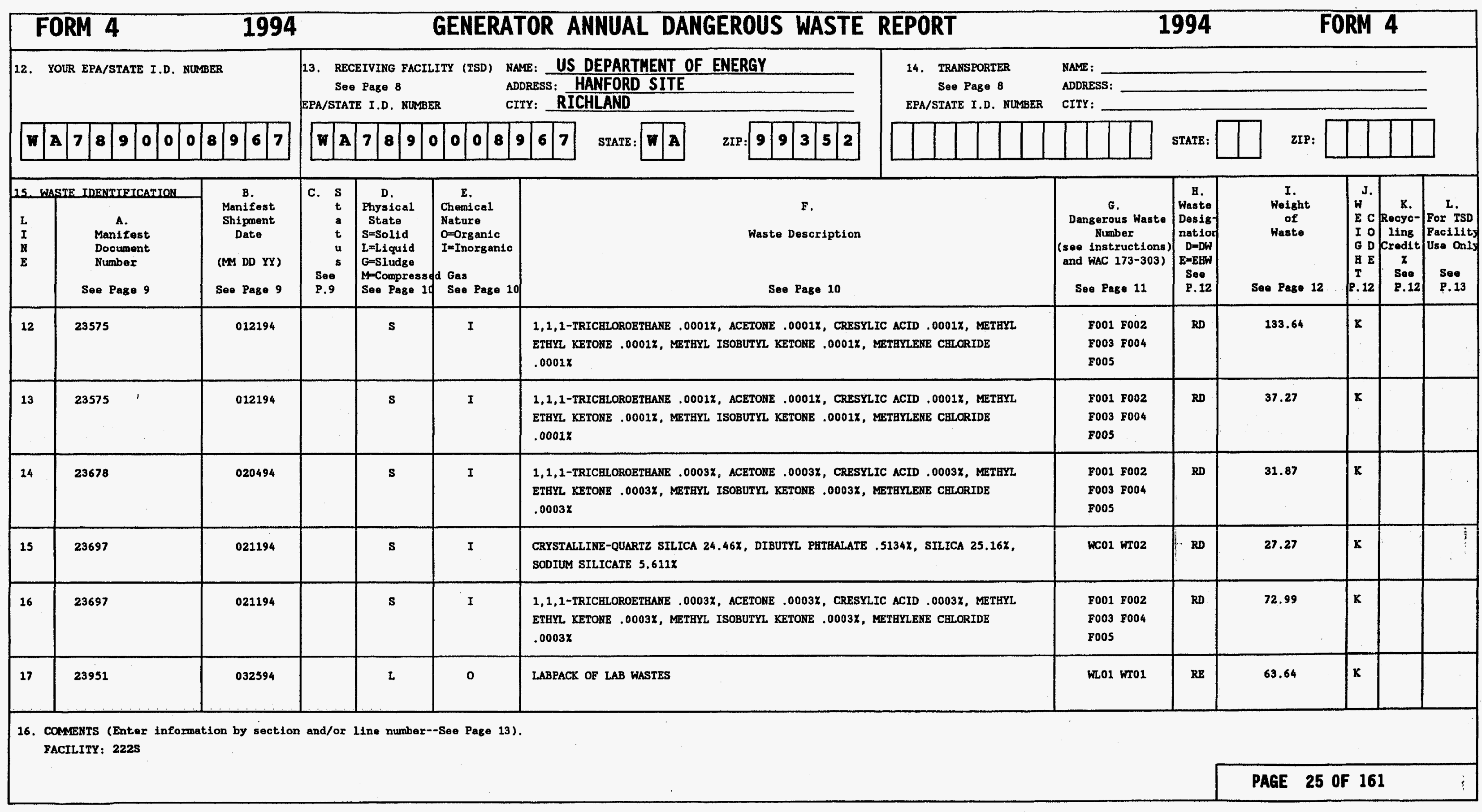




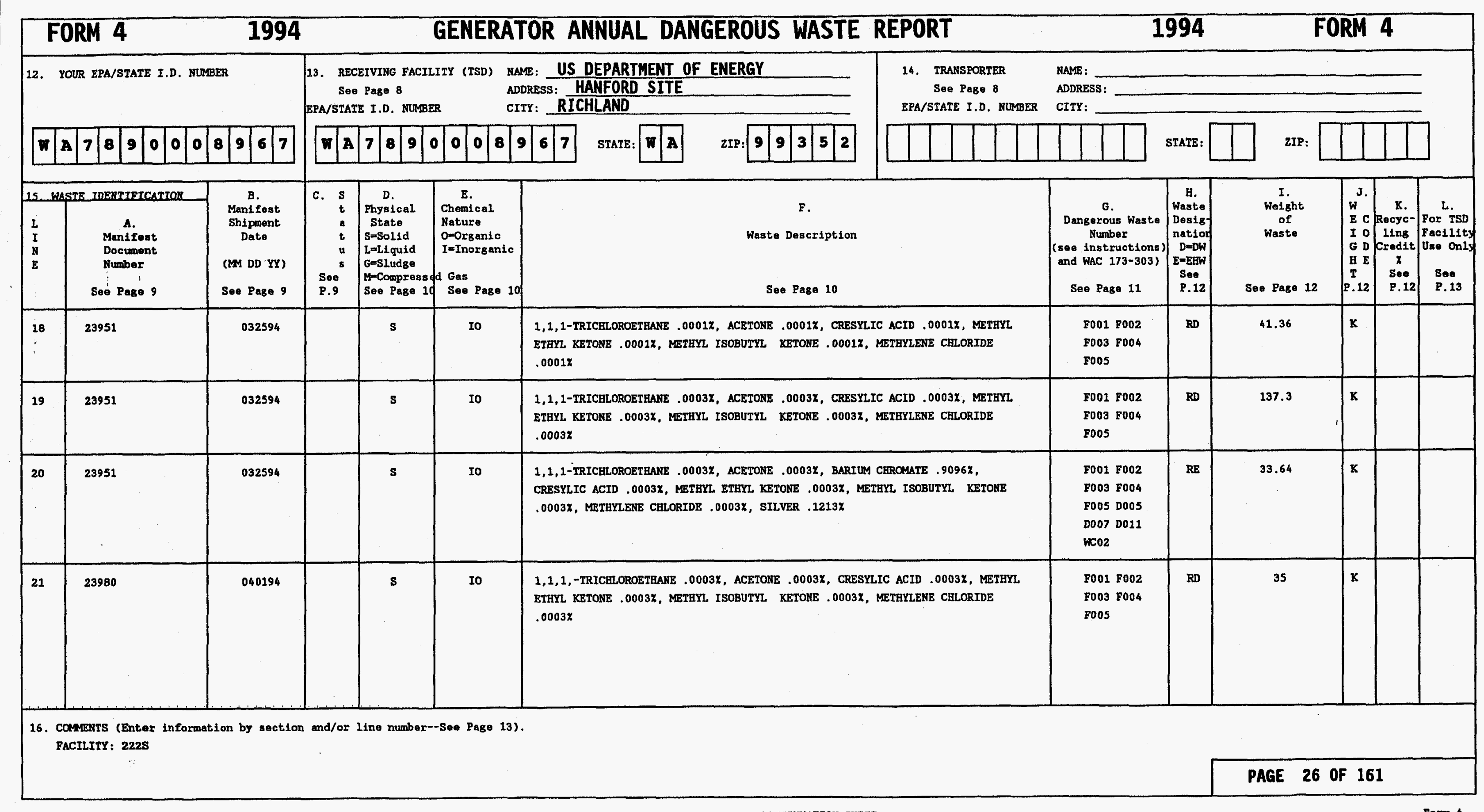




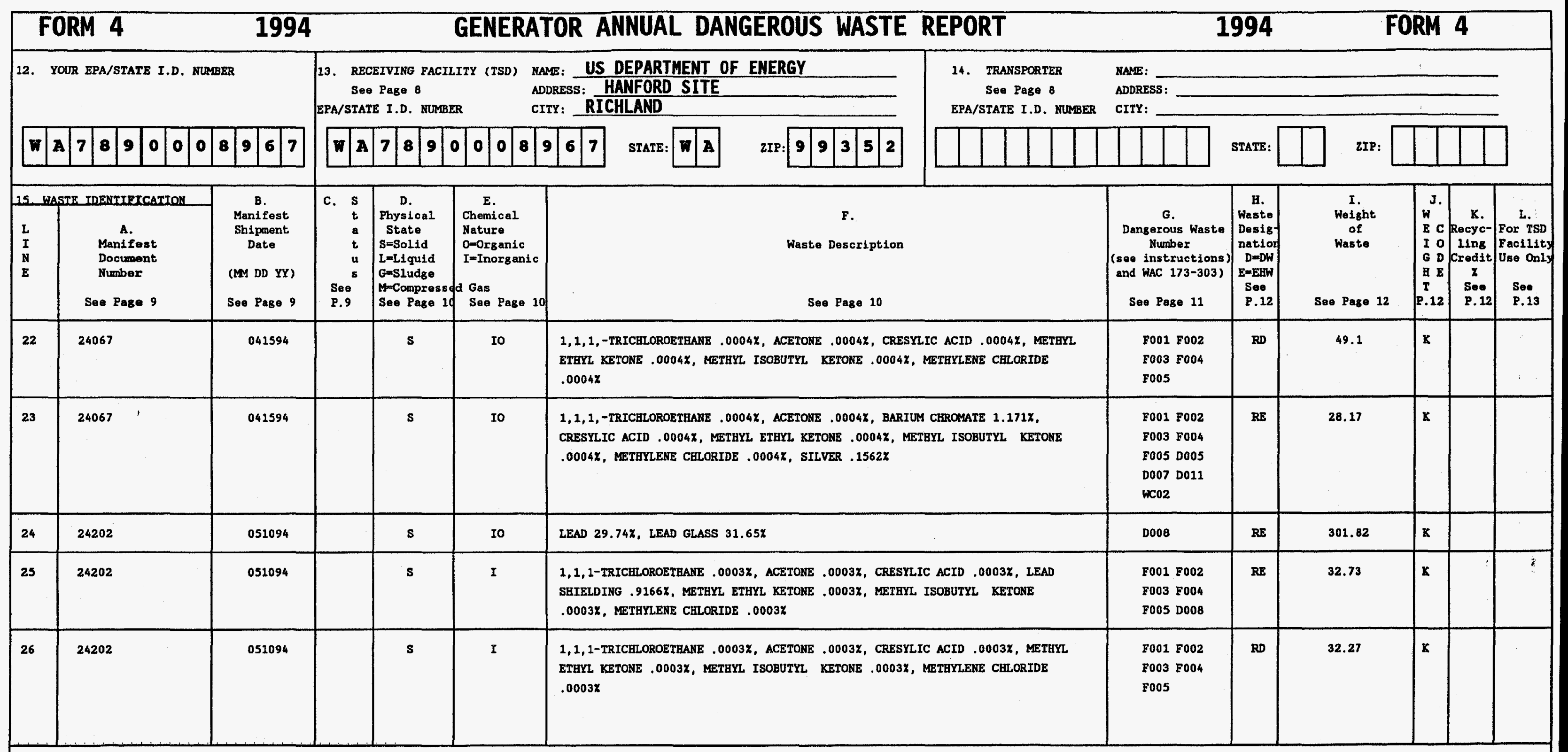

16. CaMreNIS (Enter information by section and/or line number--See Page 13). FACILITY: 222S 


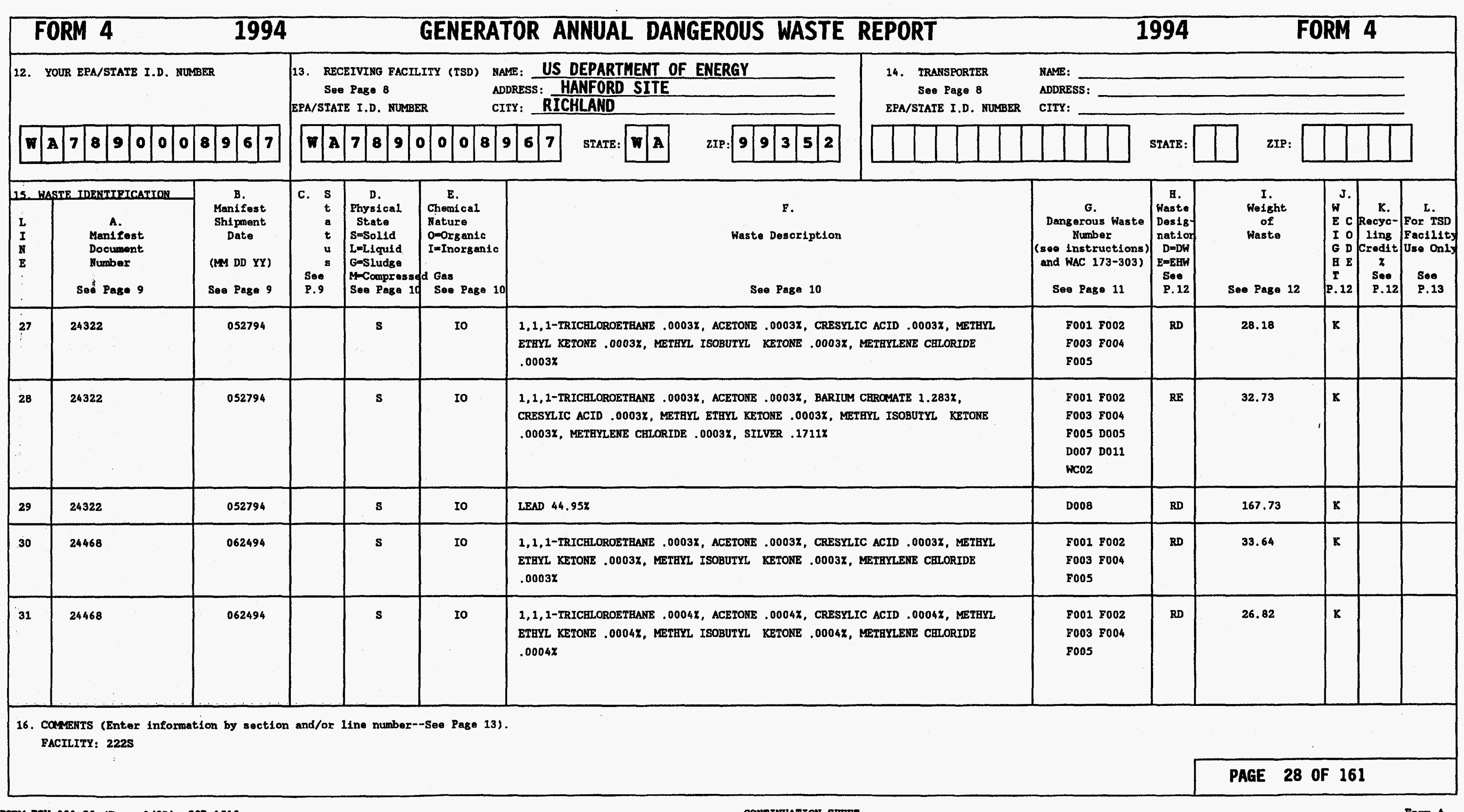




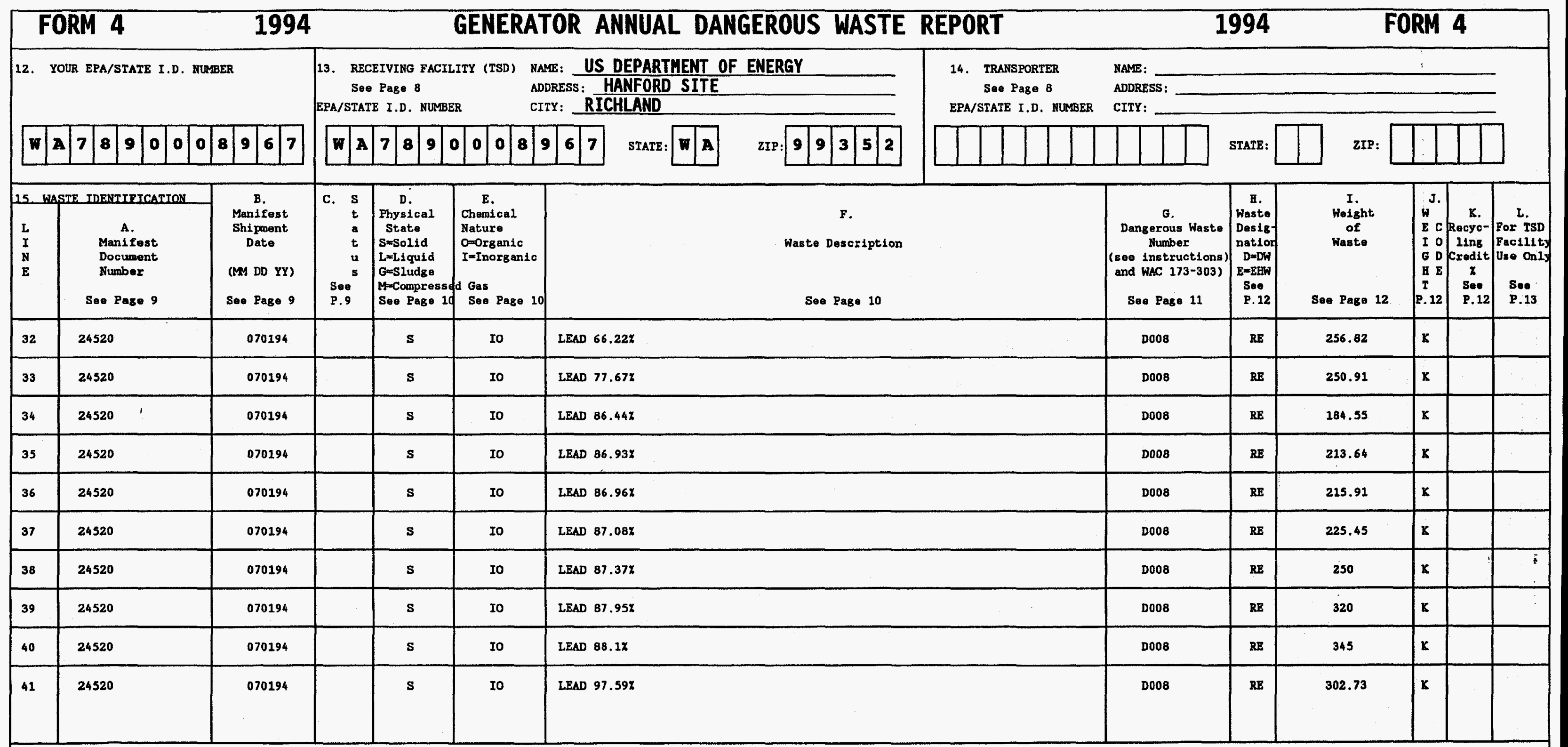

16. COMENTS (Enter information by section and/or line number--Soe Page 13). FACILITY: 222S 


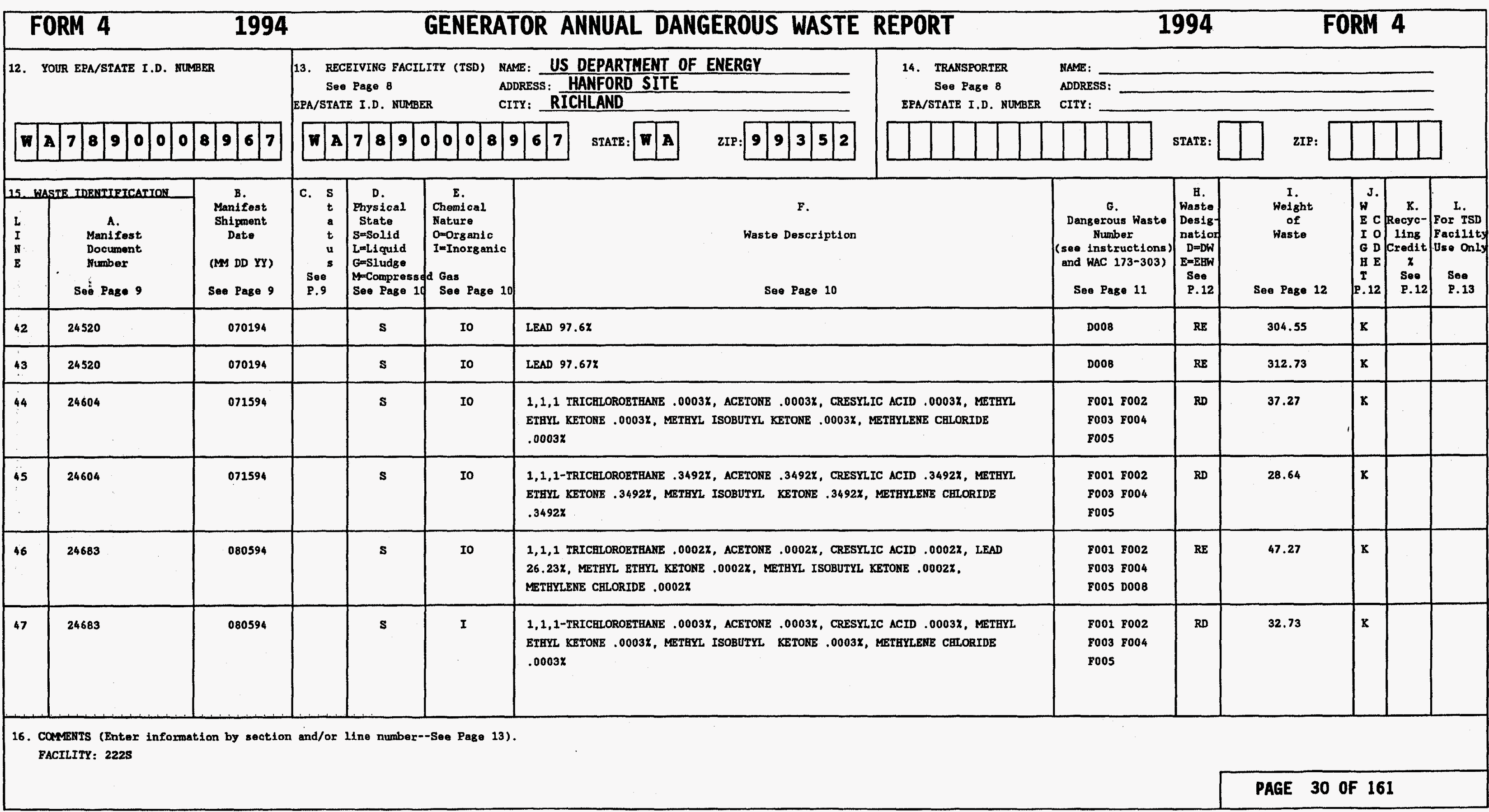




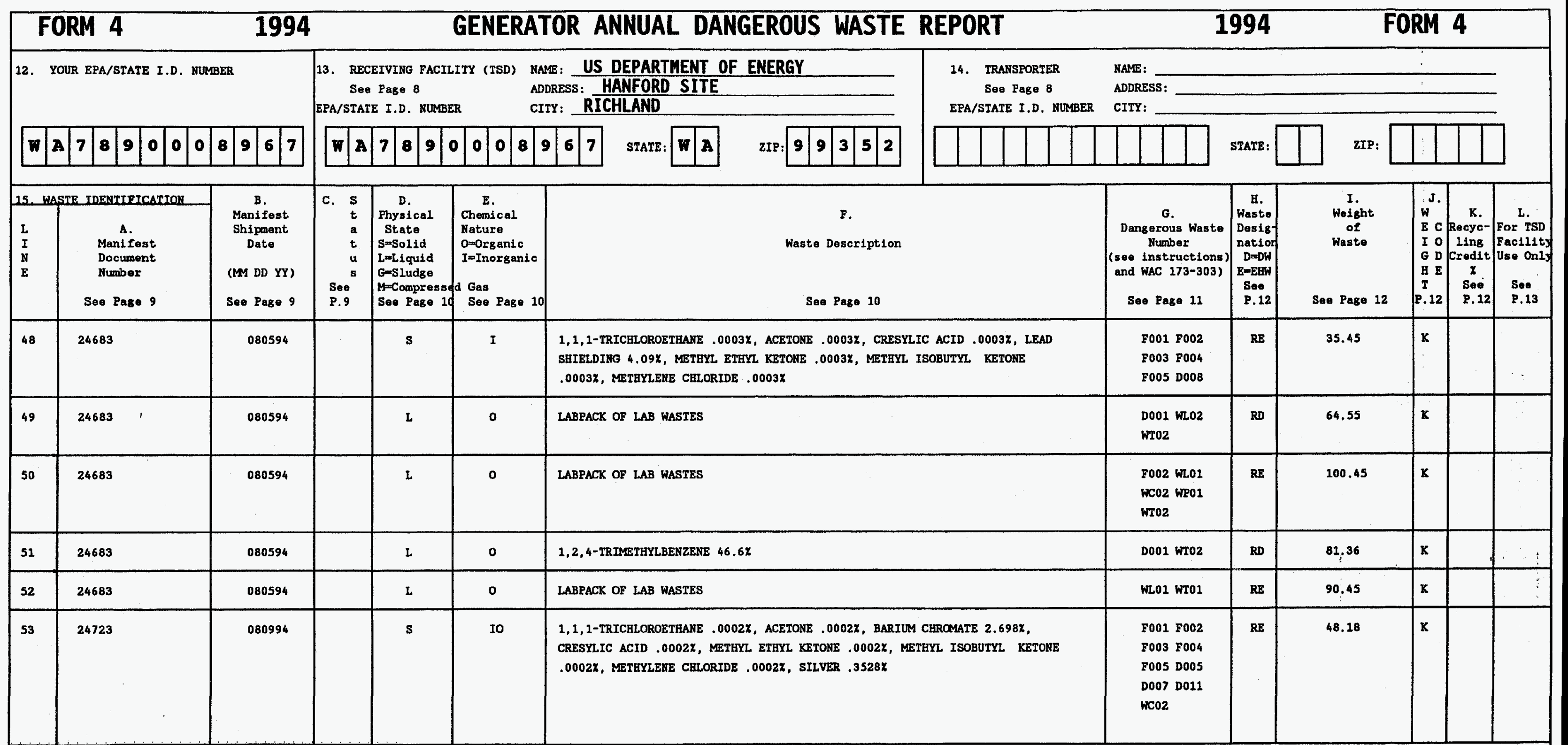

16. COMMENTS (Enter information by section and/or 11ne number--See Page 13). FACILITY: 2225

PAGE 31 OF 161 


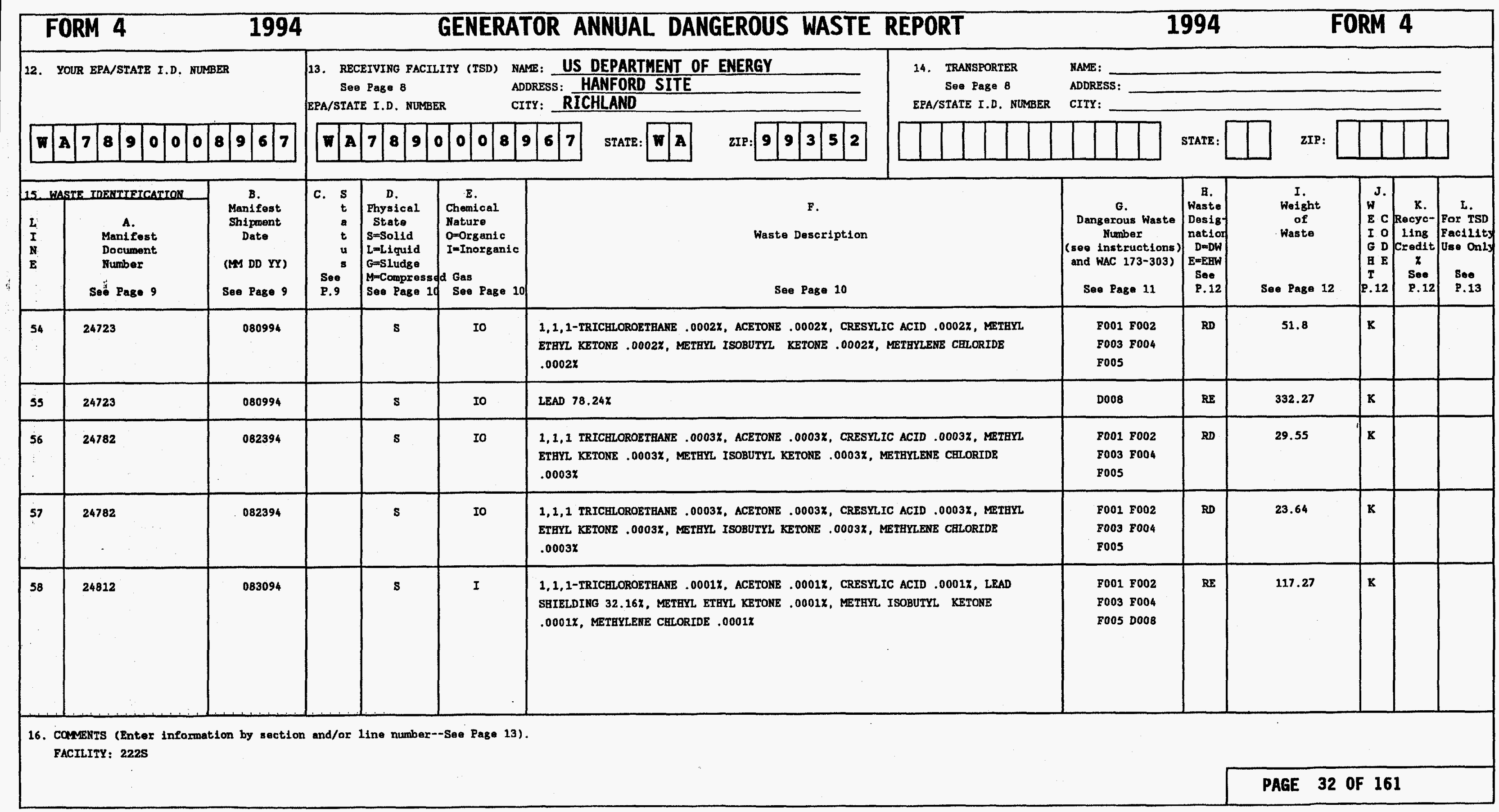




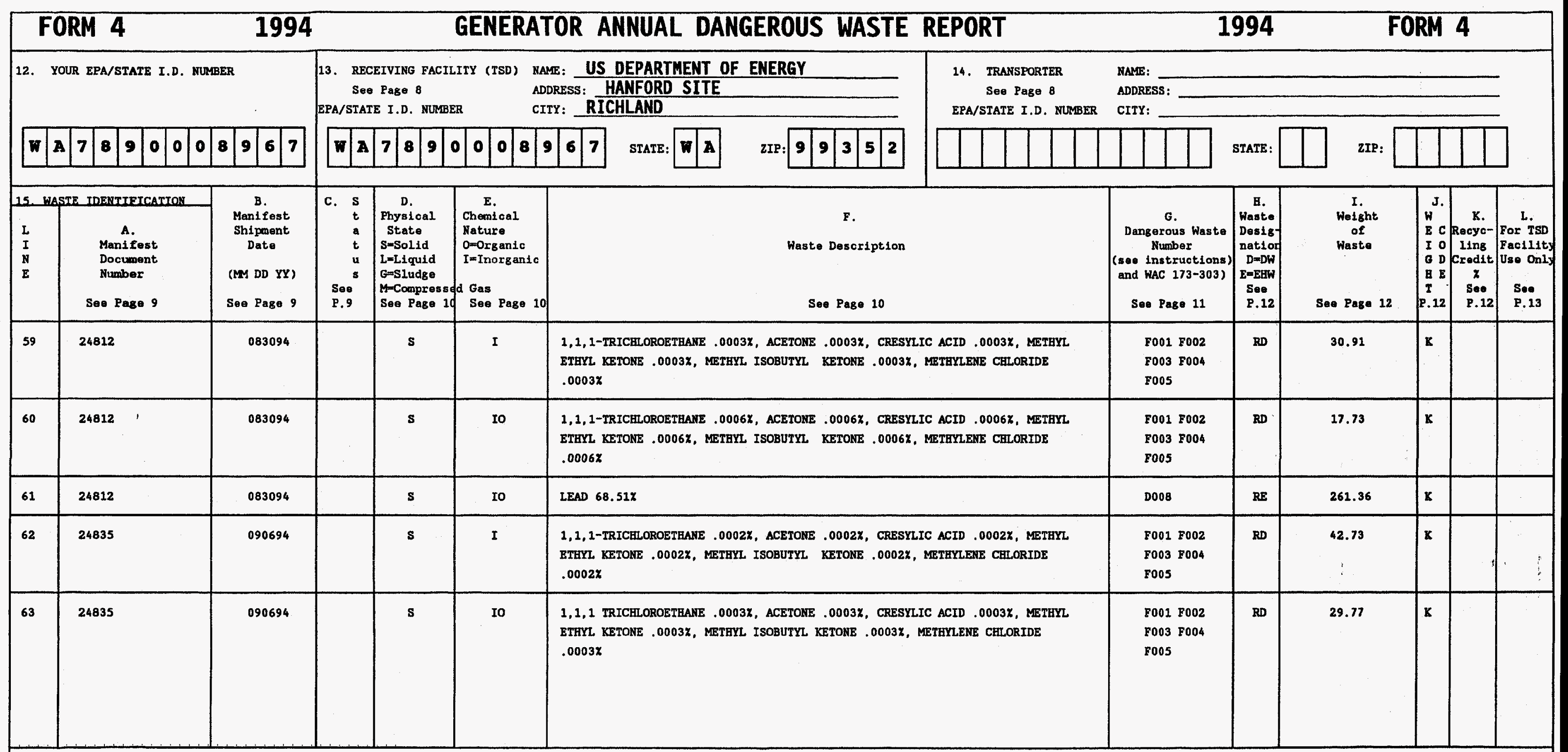

16. COMMENIS (Enter information by section and/or Iine number--See Page 13). FACILITY: 222S 


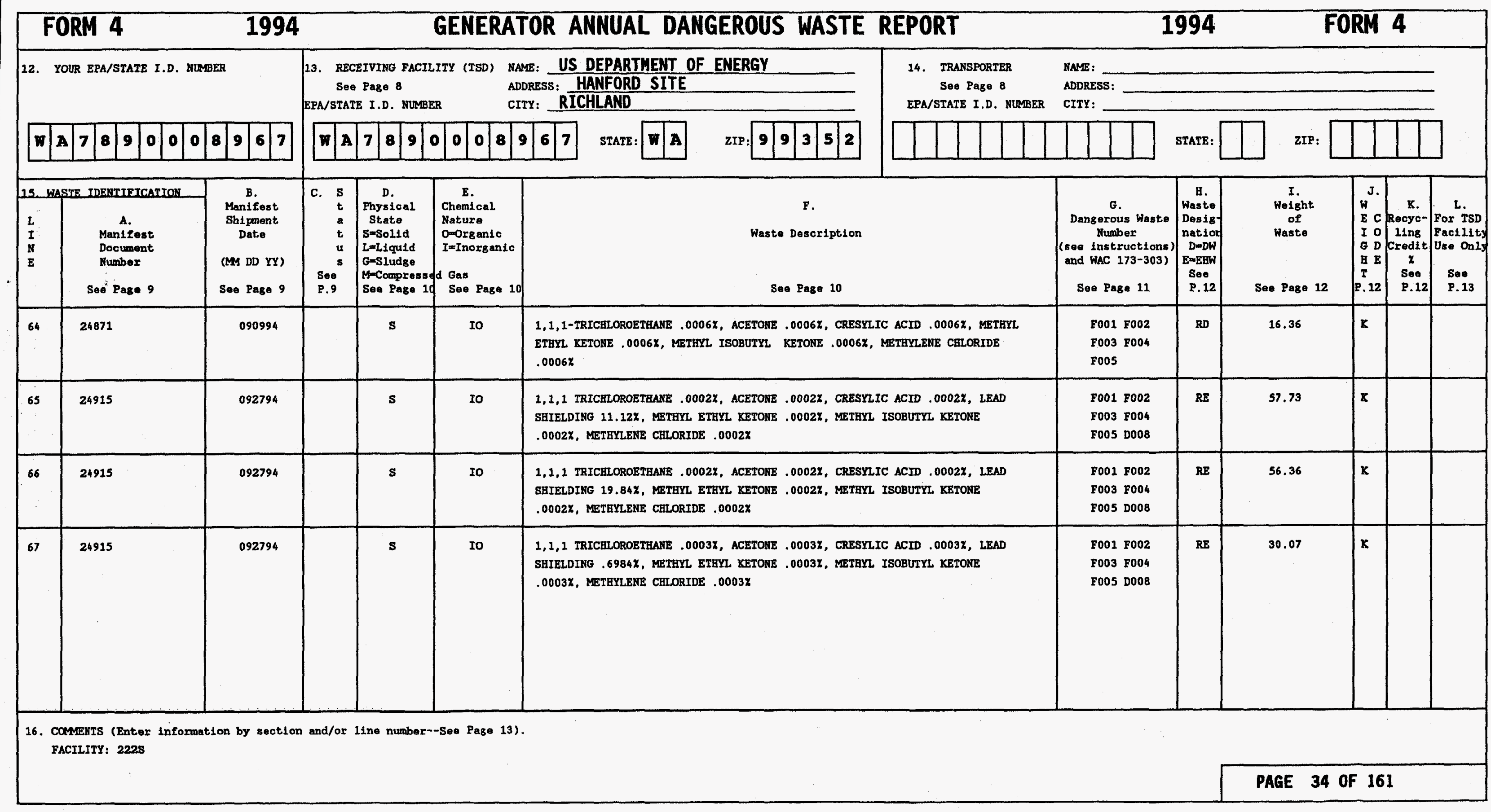




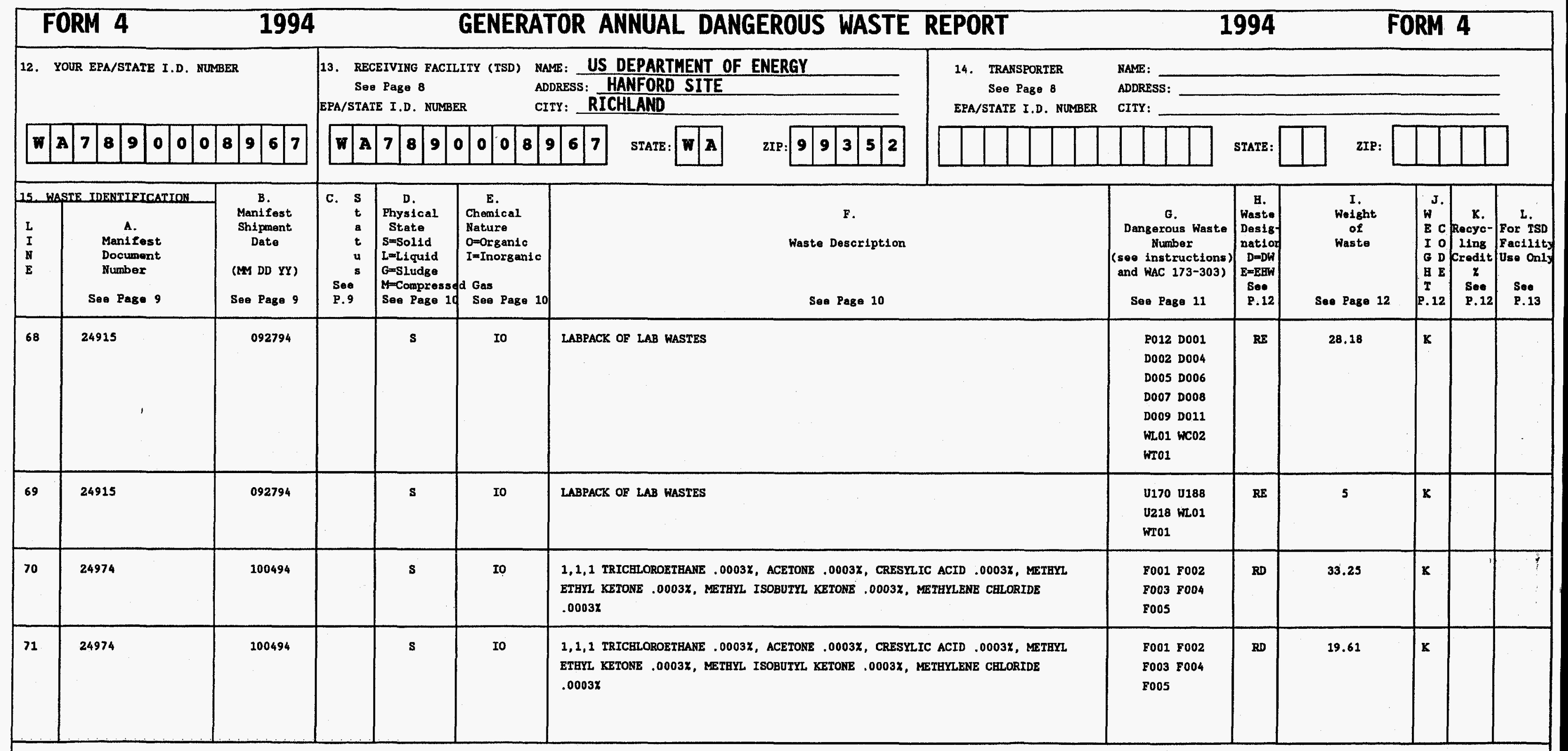

16. COMAETS (Enter information by section and/or 11ne number--See Page 13). FACILITY: 222S

PAGE 35 OF 161 


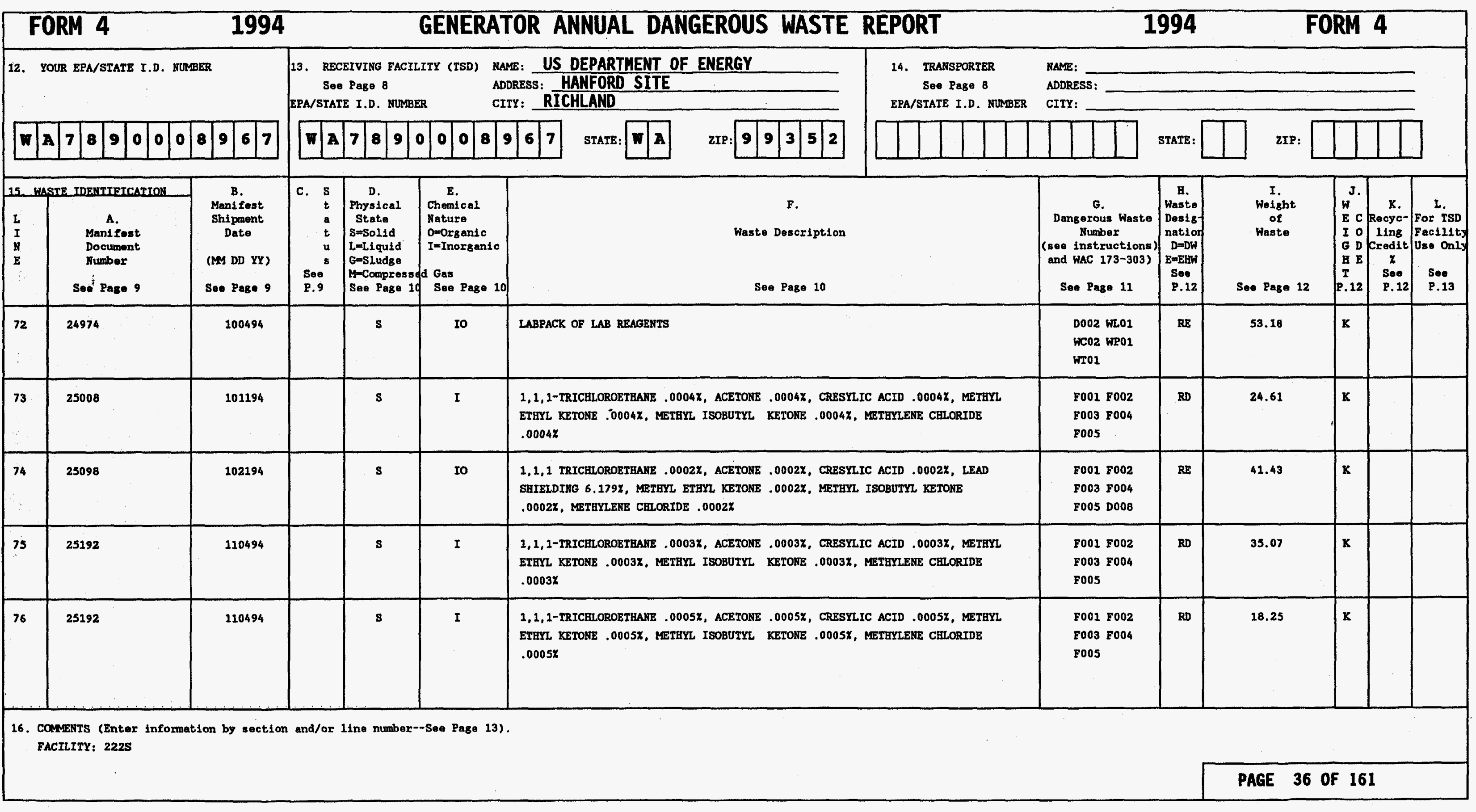




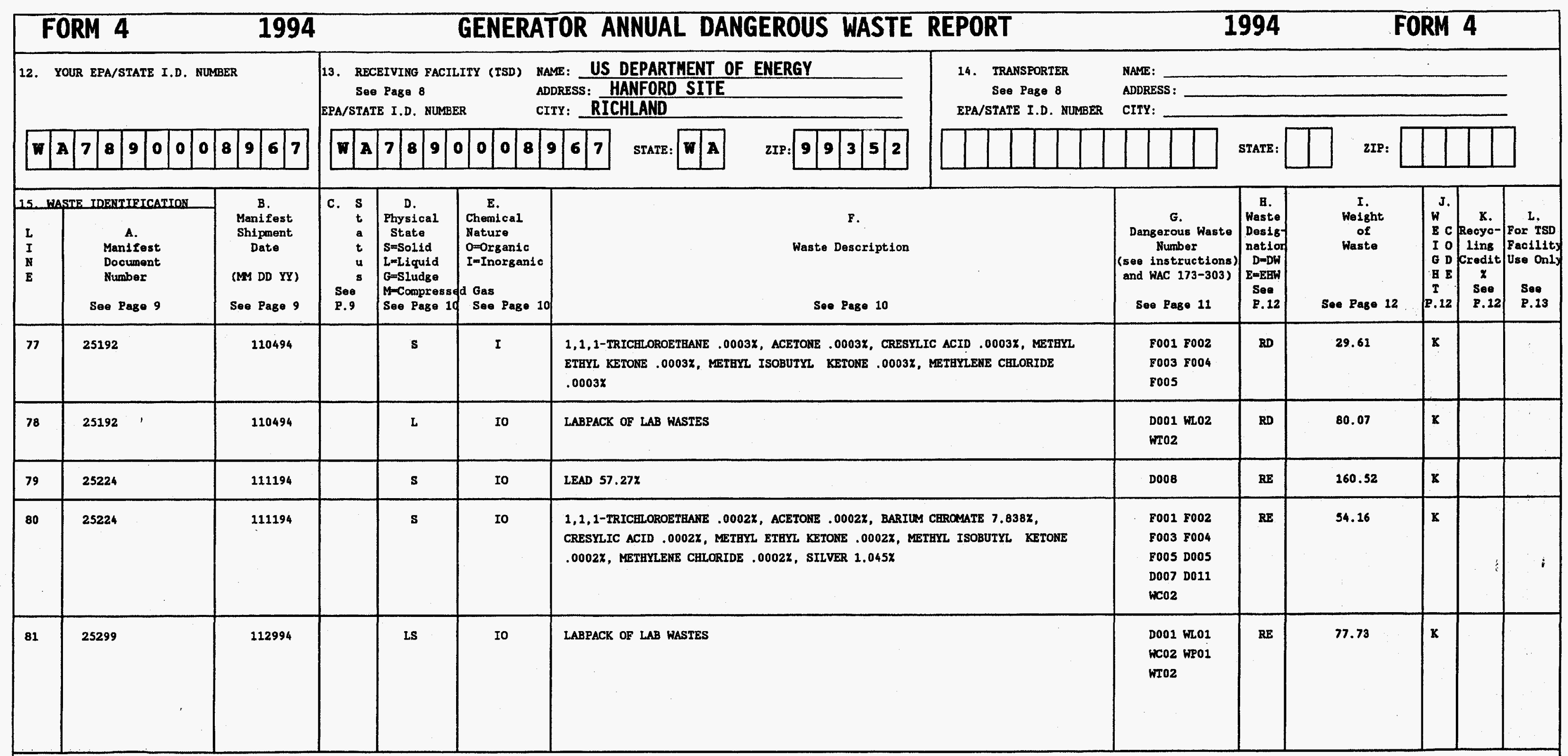

16. COMMENTS (Enter Inforration by section and/or 11ne numbor--Sae Page 13). FACILITY: 222S 


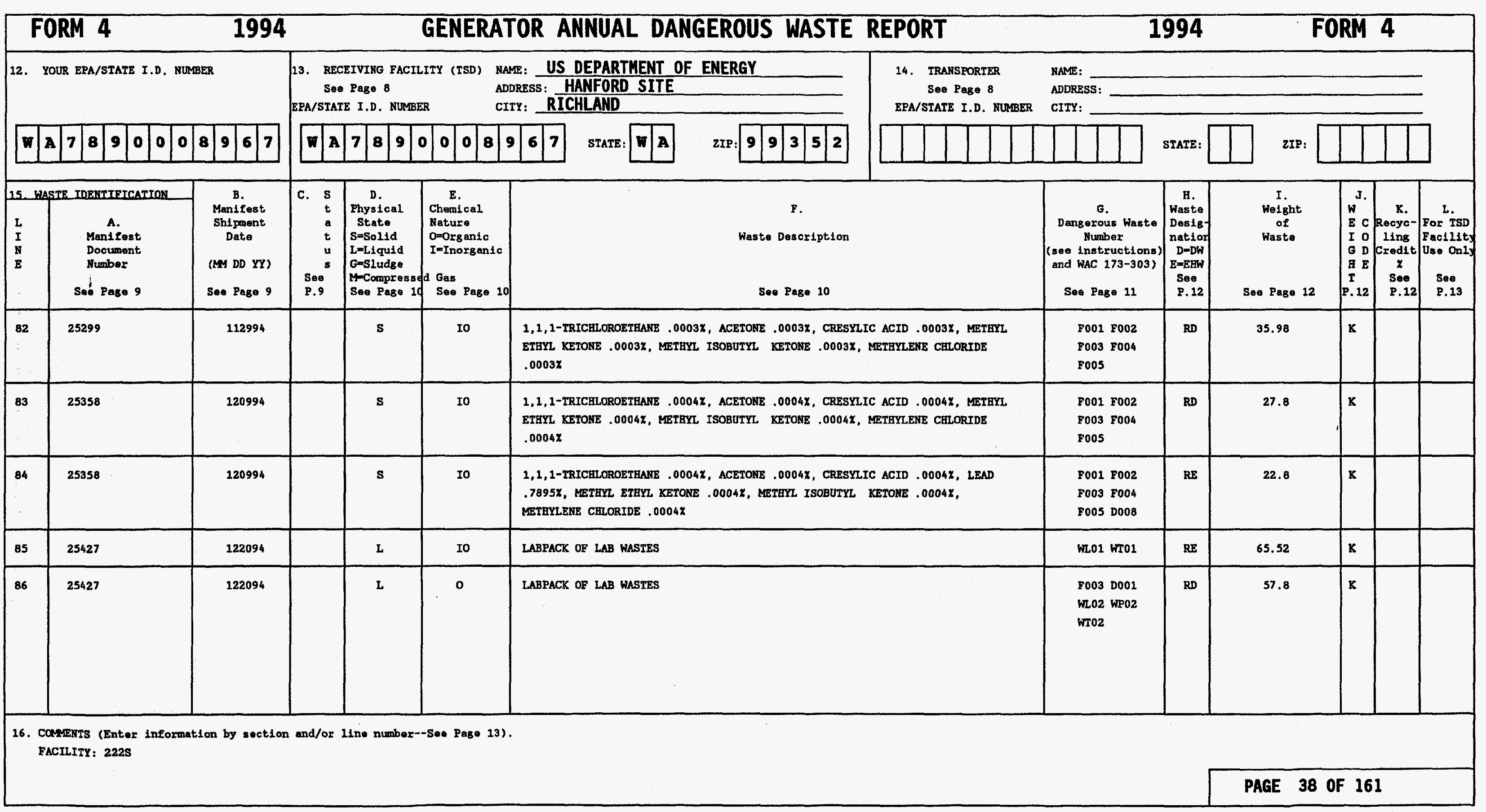




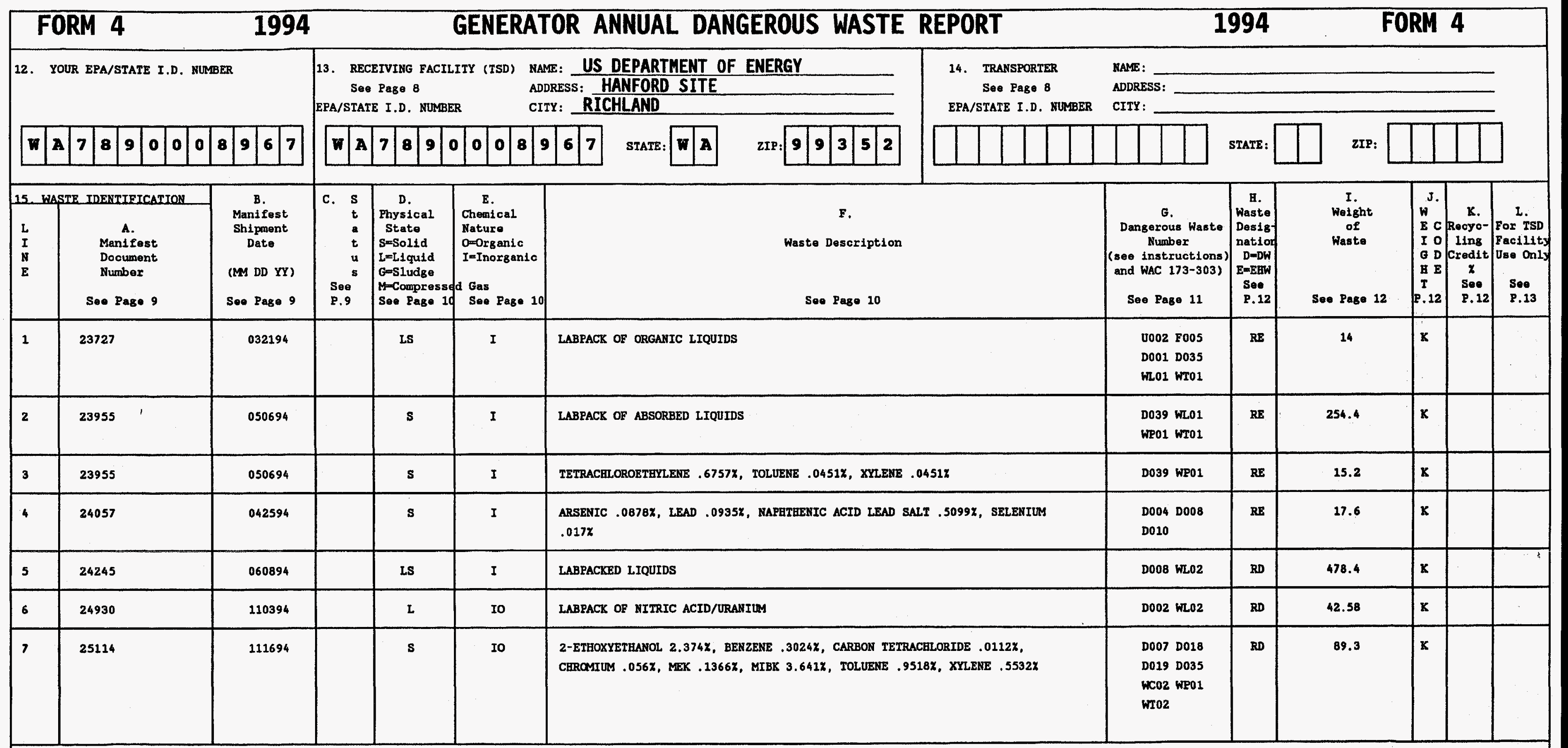

16. COMMETS (Enter information by section and/or line number--Soe Page 13). FACILITY: 224U 


\begin{tabular}{|c|c|c|c|c|c|c|c|c|c|c|c|c|c|}
\hline & $\overline{R M} 4$ & 1994 & & & GENERA & OR ANNUAL DANGEROUS WASTE & EPORT & & 994 & & $\overline{R M}$ & & \\
\hline & R EPA/STATE I.D. & & $\begin{aligned} & \text { 13. } \text { REC } \\
& \text { Seo } \\
& \text { EPA/STAI }\end{aligned}$ & $\begin{array}{l}\text { CEIVING FACIL } \\
\text { Eage } 8 \\
\text { IE I.D. NUMBE }\end{array}$ & $\begin{array}{ll}\text { LITY (TSD) } & \text { NA } \\
& A D \\
E R & C I\end{array}$ & $\begin{array}{l}\text { UE: US DEPARTMENT OF ENERGY } \\
\text { ORESS: } \frac{\text { HANFORD SITE }}{\text { RICHLAND }}\end{array}$ & $\begin{array}{l}\text { 14. TRANSPORTER } \\
\text { See Page } 8 \\
\text { EPA/STATE I.D. NUMBER }\end{array}$ & $\begin{array}{l}\text { IAME: } \\
\text { ADDRESS: } \\
\text { CITY: }\end{array}$ & & 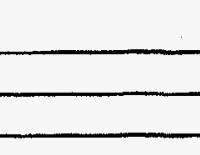 & & $\bar{E}$ & \\
\hline 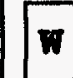 & \begin{tabular}{|l|l|l|l|l|}
7 & 8 & 9 & 0 & 0 \\
\end{tabular} & \begin{tabular}{|l|l|l|l|}
8 & 9 & 6 & 7 \\
\end{tabular} & $\mathbf{n}$ & \begin{tabular}{|l|l|l|l}
7 & 8 & 9 & 1 \\
\end{tabular} & \begin{tabular}{l|l|l|l|l}
0 & 0 & 0 & 8 & \\
\end{tabular} & 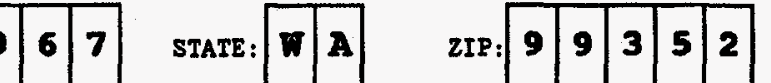 & & & STATE: & zIP: & & & \\
\hline 215. & CE SDENTIEICATION & & c. $s$ & & & & & & B. & I. & 3. & $x$ & \\
\hline $\begin{array}{l}2 \\
I \\
\mathbb{N} \\
E\end{array}$ & $\begin{array}{c}\text { A. } \\
\text { Manifest } \\
\text { Document } \\
\text { Number } \\
\text { See Page } 9\end{array}$ & $\begin{array}{l}\begin{array}{c}\text { Shipment } \\
\text { Date }\end{array} \\
\text { (MM DD YY) } \\
\text { See Page } 9\end{array}$ & $\begin{array}{c}\mathrm{a} \\
\mathrm{t} \\
\mathrm{u} \\
\mathrm{s} \\
\mathrm{s} \\
\mathrm{P} .9\end{array}$ & 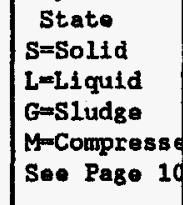 & $\begin{array}{l}\text { Nature } \\
\text { O=Organic } \\
\text { I-Inorganic } \\
\text { d Gas } \\
\text { Sao Page } 10\end{array}$ & $\begin{array}{l}\text { Waste Description } \\
\text { See Page } 10\end{array}$ & & \begin{tabular}{|c} 
Dangerous Waste \\
Number \\
(see 1nstructions) \\
and WAC 173-303) \\
Soe Page 11
\end{tabular} & $\mid \begin{array}{c}\text { Desig- } \\
\text { nation } \\
\text { D=DW } \\
\text { E=EEW } \\
\text { See } \\
\text { P.12 }\end{array}$ & $\begin{array}{c}\text { of } \\
\text { Waste } \\
\text { See Page } 12\end{array}$ & $\left|\begin{array}{ll}E & C \\
I & 0 \\
\text { G } & \text { D } \\
\text { H } & \text { E } \\
\text { E. } & \\
\text {.12 }\end{array}\right|$ & $\left|\begin{array}{c}\text { Rooyc- } \\
\text { 1ning } \\
\text { credit } \\
x \\
\text { soe } \\
\text { P.12 } \\
.12\end{array}\right|$ & $\begin{array}{l}\text { For ISD } \\
\text { Facility } \\
\text { Use Only } \\
\text { See } \\
\text { P.13 }\end{array}$ \\
\hline 8 & 25188 & 111694 & & $\mathrm{~s}$ & Io & AEPTACHLOR $x$ & & D031 & RD & 14 & $\mathrm{x}$ & & \\
\hline 9 & 25378 & 122894 & & L & 0 & LAPACK OF OILS & & $\begin{array}{l}\text { F002 F003 } \\
\text { F005 D008 } \\
\text { D040 WLO2 }\end{array}$ & RD & 654 & $\mathbf{x}$ & & \\
\hline 10 & 25378 & 122894 & & I & 0 & LABPACK OF OILS & & $\begin{array}{l}\text { F002 F003 } \\
\text { F005 D005 } \\
\text { D008 D039 } \\
\text { WL01 WP02 }\end{array}$ & $\mathrm{RE}$ & 170 & $\mathrm{x}$ & & \\
\hline 11 & 25378 & 122894 & & I & 0 & LAPACK OF OLLS & & $\begin{array}{l}\text { F002 F003 } \\
\text { F005 D005 } \\
\text { D008 D039 } \\
\text { WL01 WP02 }\end{array}$ & $\mathrm{RE}$ & 75 & $\mathrm{x}$ & & \\
\hline & $\begin{array}{l}\text { ITS (Entor in } \\
\text { ITY: 224U }\end{array}$ & . & & & a Page & & & & & & & & \\
\hline & & & & & & & & & & PAGE 40 & F 16 & & \\
\hline
\end{tabular}




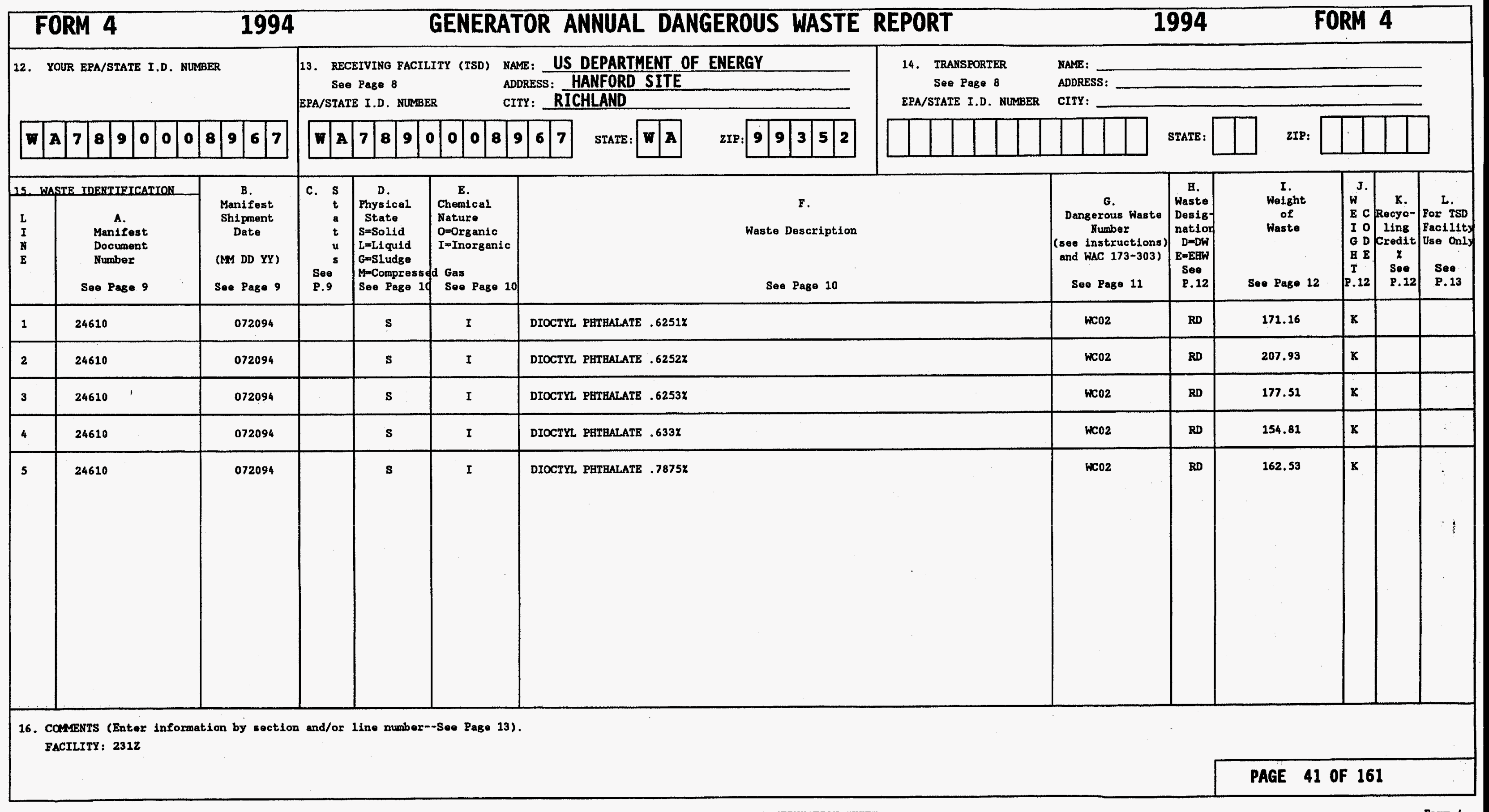




\begin{tabular}{|c|c|c|c|c|c|c|c|c|c|c|c|c|c|}
\hline & RM 4 & 1994 & & & GENERA1 & OR ANNUAL DANGEROUS WASTE & EPORT & & 994 & & $\overline{R M}$ & & \\
\hline & UR EPA/STATE I.D. & & 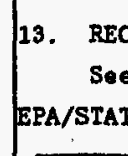 & $\begin{array}{l}\text { EIVTNG FACIL: } \\
\text { Page } 8 \\
\text { E I.D. NUMBEI }\end{array}$ & $\begin{array}{ll}\text { LITY (TSD) } & \text { NAI } \\
\text { ER } & \text { ADI }\end{array}$ & $\begin{array}{l}\text { 1E: US DEPARTMENT OF ENERGY } \\
\text { DRESS: } \frac{\text { HANFORD SITE }}{\text { RICHLAND }} \\
\mathrm{YYN}\end{array}$ & $\begin{array}{l}\text { 14. TRANSPORTER } \\
\text { See Page } 8 \\
\text { EPA/STATE I.D. NUMBER }\end{array}$ & $\begin{array}{l}\text { NAME: } \\
\text { ADDRESS: } \\
\text { CITY: }\end{array}$ & & 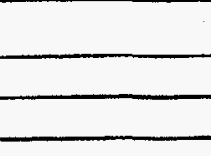 & & 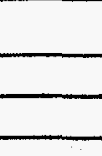 & \\
\hline$n$ & \begin{tabular}{|l|l|l|l|l|}
7 & 8 & 9 & 0 & 0 \\
\end{tabular} & \begin{tabular}{|l|l|l|l|}
8 & 9 & 6 & 7 \\
\end{tabular} & \begin{tabular}{|l|l|}
$\mathbf{w}$ & $\mathbf{A}$ \\
\end{tabular} & \begin{tabular}{|l|l|l|l|}
7 & 8 & 9 & 0 \\
\end{tabular} & \begin{tabular}{l|l|l|l|l}
0 & 0 & 0 & 8 & 5
\end{tabular} & 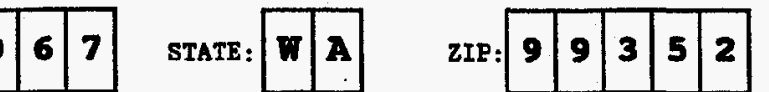 & & & STATE: & 2IP: & & & \\
\hline 15. & TE IDENTretcatron & & c. $s$ & & & & & & в. & I. & J. & & \\
\hline $\begin{array}{l}\mathrm{L} \\
\mathrm{I} \\
\mathrm{I} \\
\mathrm{E}\end{array}$ & $\begin{array}{l}\text { A. } \\
\text { Manifest } \\
\text { Document } \\
\text { Number } \\
\text { Só́ Page } 9 \\
\end{array}$ & $\begin{array}{c}\text { Shipment } \\
\text { Date } \\
\text { (MM DD YY) } \\
\text { See Page } 9 \\
\end{array}$ & $\begin{array}{r}t \\
t \\
u \\
\text { s } \\
\text { Sea } \\
\text { P.9 } \\
\end{array}$ & 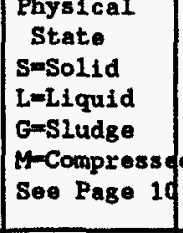 & $\begin{array}{l}\text { Chemical } \\
\text { Nature } \\
\text { O-Organic } \\
\text { I=Inorganic } \\
\text { ol Gas } \\
\text { See Page } 10\end{array}$ & $\begin{array}{l}\text { Waste Description } \\
\text { See Page } 10\end{array}$ & & $\begin{array}{c}\text { G. } \\
\text { Dangerous Waste } \\
\text { Number } \\
\text { (see Instructions) } \\
\text { and WAC 173-303) } \\
\text { SoO Page } 11\end{array}$ & 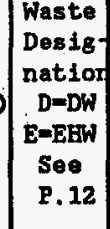 & $\begin{array}{c}\begin{array}{c}\text { Weight } \\
\text { of } \\
\text { Waste }\end{array} \\
\text { SoP Page } 12\end{array}$ & 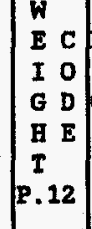 & 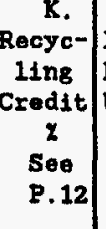 & 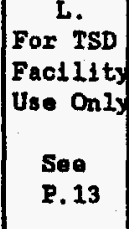 \\
\hline 1 & 23275 & 012594 & & Ls & o & LABPACK OF ORGANIC SOLUTIONS & & $\begin{array}{l}\text { Do01 D035 } \\
\text { WL01 WT01 }\end{array}$ & RE & 15 & $\mathrm{x}$ & & \\
\hline 2 & 23305 & 011494 & & 2 & 0 & LABPACK OF PAINTS & & $\begin{array}{l}\text { U002 U161 } \\
\text { D001 D007 } \\
\text { D035 WL01 } \\
\text { WC02 WP01 } \\
\text { Wr01 }\end{array}$ & RE & 47 & k & & \\
\hline 3 & 23306 & 012594 & & L & ro & LABPACK OF EXPTRED CCMMERCIAL PRODUCTS & & $\begin{array}{l}\text { WL01 HCO1 } \\
\text { WP01 WT01 }\end{array}$ & $\mathrm{RE}$ & 24 & $\mathrm{x}$ & & \\
\hline 4 & 23306 & 012594 & & L & ro & LABPACK OF EXPIRED CONMERCIAL FRODUCTS & & WL01 wro1 & $\mathrm{RE}$ & 15 & $\mathbf{x}$ & & \\
\hline 5 & 23306 & 012594 & & $\mathbf{s}$ & to & LABPACX OF EXPIRED COMMRCIAL PRODUCTS & & $\begin{array}{l}\text { WLO1 WCO1 } \\
\text { WT01 }\end{array}$ & $\mathrm{RE}$ & 15 & $\mathbf{x}$ & & \\
\hline & $\begin{array}{l}\text { S (Enter inf } \\
X: 23452\end{array}$ & yy sec & $1 / 0$ & number-- & -See Page & & & & & & & & \\
\hline & & & & & & & & & & PAGE 42 & F 161 & & \\
\hline
\end{tabular}




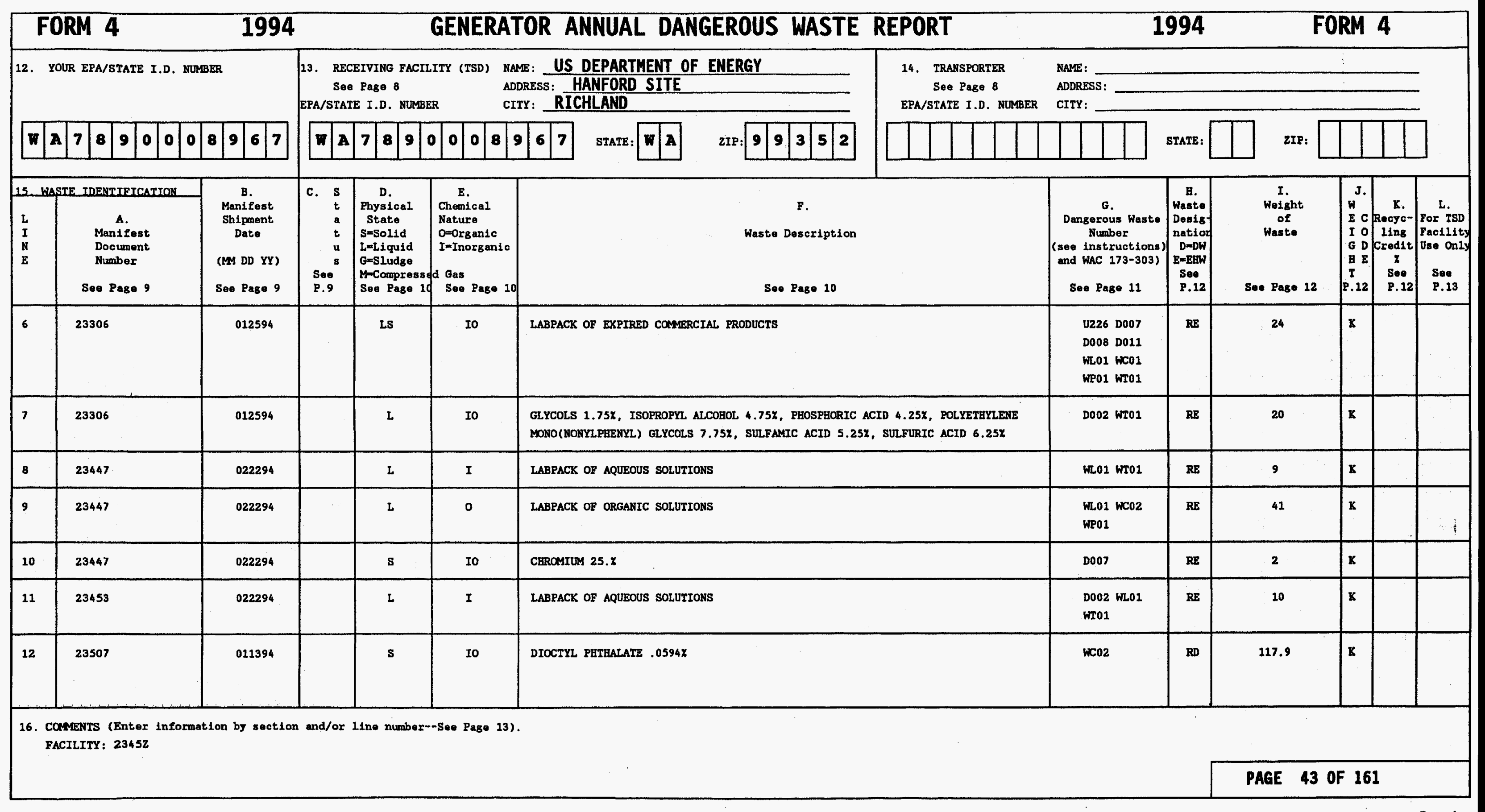




\begin{tabular}{|c|c|c|c|c|c|c|c|c|c|c|c|c|c|}
\hline \multicolumn{2}{|c|}{ FORM 4} & 1994 & \multicolumn{5}{|c|}{ GENERATOR ANNUAL DANGEROUS WASTE REPORT } & \multicolumn{2}{|c|}{1994} & \multicolumn{3}{|c|}{ FORM 4} & \\
\hline \multicolumn{3}{|c|}{ 12. YOUR EPA/STATE Y.D. NUMBER } & \multicolumn{4}{|c|}{$\begin{array}{ll}\text { 13. RECEIVING FACLIITY (TSD) } & \text { NAME: } \\
\text { See Page \& DEPARTMENT OF ENERGY } & \text { ADDRESS: HANFORD SITE } \\
\text { EPA/STAIE I.D. NUMBER } & \text { CITY: RICHLAND } \\
\end{array}$} & $\begin{array}{l}\text { 14. TRANSPORTER } \\
\text { Sea Page } 8 \\
\text { EPA/STATE I.D. NUMBER }\end{array}$ & \multicolumn{5}{|c|}{$\begin{array}{l}\text { IAME: } \\
\text { ADDRESS: } \square \\
\text { CITY: }\end{array}$} & \\
\hline $\mathbf{m}$ & \begin{tabular}{l|l|l|l|l|l}
7 & 8 & 9 & 0 & 0 \\
\end{tabular} & \begin{tabular}{l|l|l|l}
8 & 9 & 6 & 7
\end{tabular} & $\mathbf{A}$ & \begin{tabular}{l|l|l|l}
7 & 8 & 9 & 0
\end{tabular} & \begin{tabular}{l|l|l|l|l}
0 & 0 & 0 & 8 & 9
\end{tabular} & 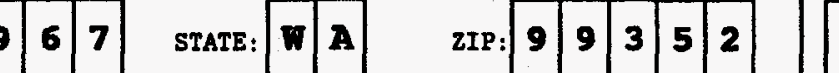 & & & staIs: & ZIP: & & & \\
\hline \multicolumn{2}{|c|}{ 15. WASTE TDENTIETCATION } & \multirow{2}{*}{ 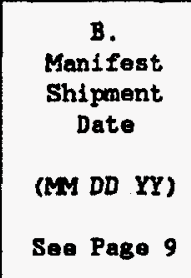 } & \multirow{2}{*}{\begin{tabular}{|c|} 
c. $s$ \\
$t$ \\
$a$ \\
$t$ \\
$u$ \\
$u$ \\
Sea \\
P.9
\end{tabular}} & \multirow{2}{*}{\begin{tabular}{|c|} 
\\
Dh. \\
Physical \\
State \\
S=Solid \\
L=Liquid \\
$G=$ Sludge \\
W-Compress \\
See Page 10
\end{tabular}} & \multirow{2}{*}{\begin{tabular}{|l|}
\multicolumn{1}{|c|}{ E. } \\
Chemical \\
Nature \\
O=Organic \\
I=Inorganic \\
d Gas \\
Soe Page 10
\end{tabular}} & \multirow{2}{*}{\multicolumn{2}{|c|}{$\begin{array}{c}\text { F. } \\
\text { Waste Description }\end{array}$}} & \multirow{2}{*}{\begin{tabular}{|c|} 
G. \\
$\begin{array}{c}\text { Dangerous Waste } \\
\text { Number } \\
\text { (see } \\
\text { Instructions) } \\
\text { and } \text { WAC } 173-303 \text { ) } \\
\text { Soe Page } 11\end{array}$ \\
\end{tabular}} & \multirow{2}{*}{ 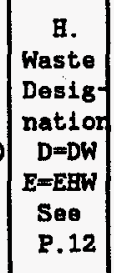 } & \multirow{2}{*}{$\begin{array}{c}\begin{array}{c}\text { I. } \\
\text { Woight } \\
\text { of } \\
\text { Waste }\end{array} \\
\text { See Page } 12\end{array}$} & \multirow{2}{*}{$\mid$\begin{tabular}{ll|} 
& $S$ \\
$W$ \\
$E$ \\
$E$ & $C$ \\
$I$ & 0 \\
$G$ & $D$ \\
$E$ & $E$ \\
$T$ \\
$T .12$
\end{tabular}} & \multirow{2}{*}{ 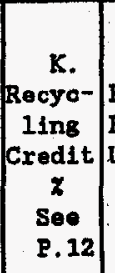 } & \multirow{2}{*}{ 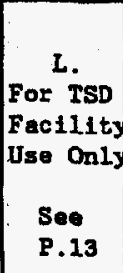 } \\
\hline $\begin{array}{l}\mathrm{L} \\
\mathrm{I} \\
\mathrm{N} \\
\mathrm{E}\end{array}$ & $\begin{array}{c}\text { A. } \\
\text { Manilest } \\
\text { Document } \\
\text { Number } \\
\text { Sobै Page } 9\end{array}$ & & & & & & & & & & & & \\
\hline 13 & 23514 & 022394 & & Ls & o & LABPACK OF PAINT MAIERIAIS & & $\begin{array}{l}\text { D001 D007 } \\
\text { D018 D035 } \\
\text { WLO1 WCO1 } \\
\text { WTO1 }\end{array}$ & $\mathrm{RE}$ & 38.85 & $\mathrm{x}$ & & \\
\hline 14 & 23810 & 042894 & & L & Io & CARBON TETRACHLORIDE $20 . x$, TRIBUTYL PHOSPHATE $10.91 x$ & & $\begin{array}{l}\text { D019 wCO2 } \\
\text { WR01 WTO2 }\end{array}$ & $\mathrm{RE}$ & 165 & $\mathrm{x}$ & & \\
\hline 15 & 23810 & 042894 & & L & ro & CARBON TETRACHORIDE 24.24X, TRIBUTYL PHOSPEATE $12.12 \mathrm{X}$ & & $\begin{array}{l}\text { DO19 WCO2 } \\
\text { WP01 HTO2 }\end{array}$ & $\mathrm{RE}$ & 33 & $\mathrm{x}$ & & \\
\hline 16 & 23810 & 042894 & & L & 0 & CARBON TETRACHLORIDE $19.61 X$, TRIBUTYL. PHOSPBATE $11.76 \mathrm{x}$ & & $\begin{array}{l}\text { Do19 WCO2 } \\
\text { WPO1 Wro2 }\end{array}$ & $\mathrm{RE}$ & 51 & $\mathrm{x}$ & & \\
\hline 17 & 23810 & 042894 & & L & o & CARBON TETRACHLORIDE 19.64X, TRIBUTYL PHOSPAATE $10.71 \mathrm{Z}$ & & $\begin{array}{l}\text { D019 WCO2 } \\
\text { WPO1 WTO2 }\end{array}$ & $\mathrm{RE}$ & 56 & $\mathrm{x}$ & & \\
\hline 18 & 23810 & 042894 & & L & o & CARBON TETRACALORIDE $20 . x$, TRIBUTYL PHOSPHATE $10.91 \mathrm{X}$ & & $\begin{array}{l}\text { DO19 hCO2 } \\
\text { WPO1 wTO2 }\end{array}$ & $\mathrm{RE}$ & s5 & $\mathbf{x}$ & & \\
\hline \multicolumn{14}{|c|}{$\begin{array}{l}\text { 16. Comients (Enter information by section and/ox 11ne number--Soe Page 13). } \\
\text { FACIIITY: } 2345 Z\end{array}$} \\
\hline & & & & & & & & & & PAGE 44 & 16 & & \\
\hline
\end{tabular}




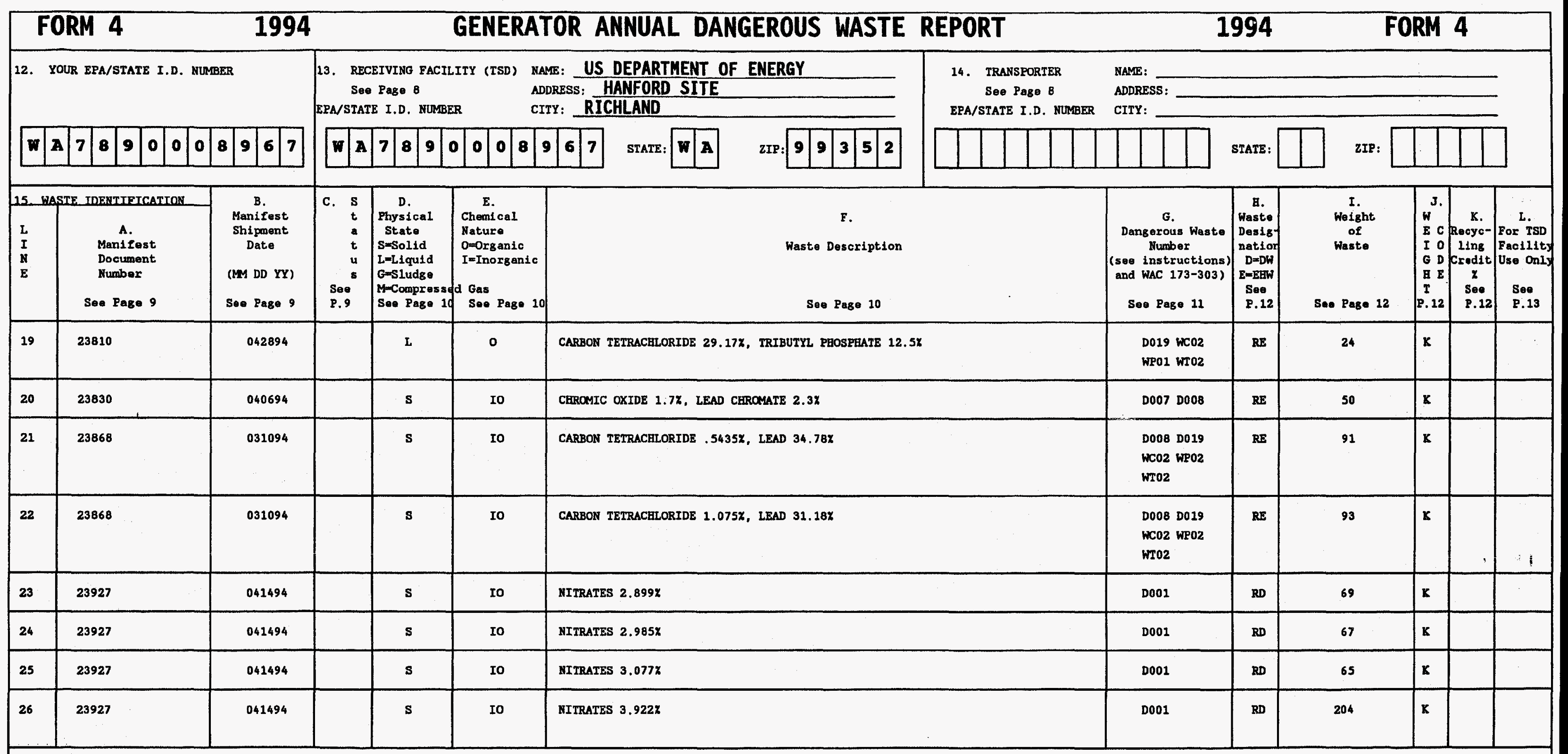

16. CameNis (Entor information by section and/or 1ine number--So• Page 13). FACILITY: 2345Z 


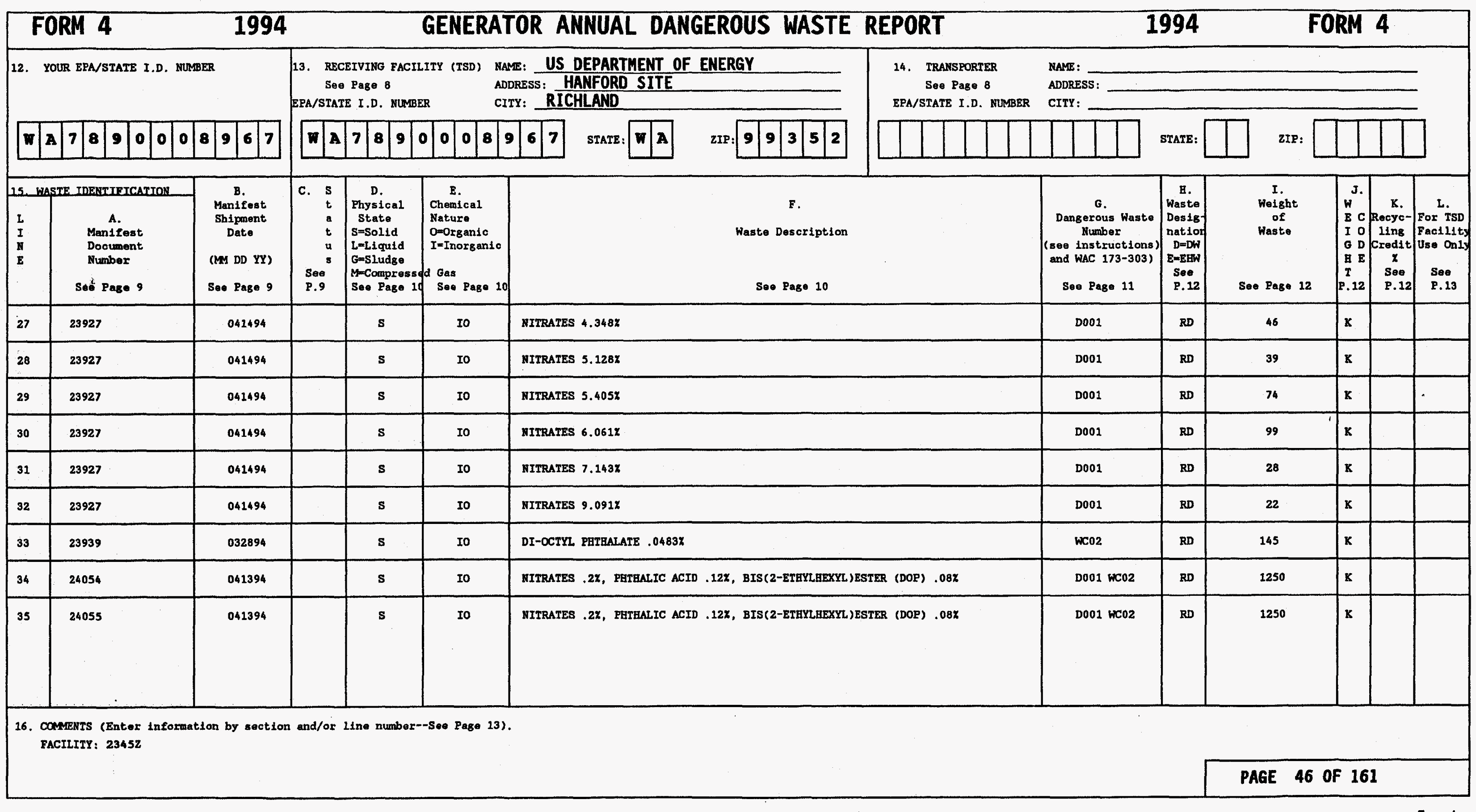




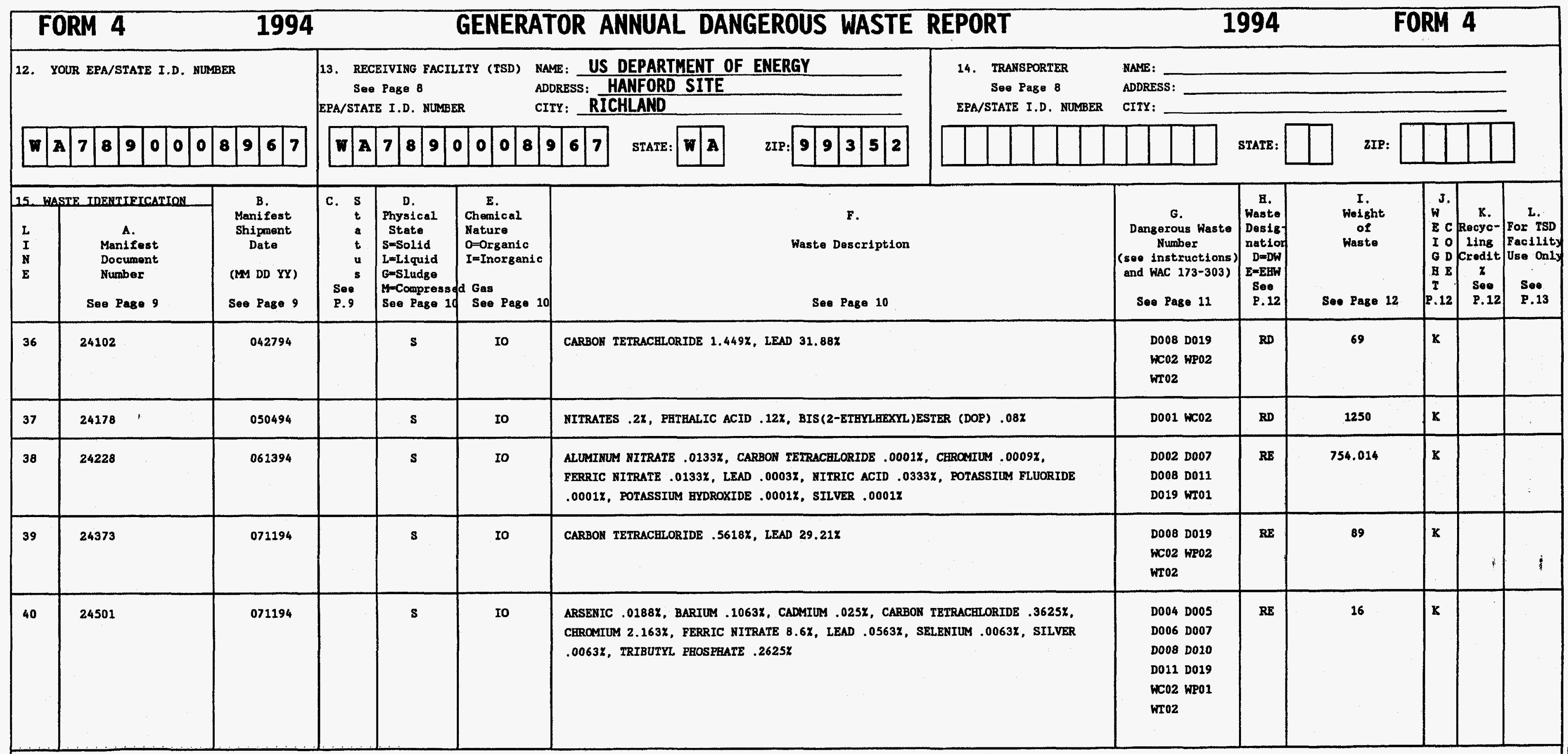

16. Comenrs (Enter information by section and/ox Line number--Sae Page 13). FACILITY: 23452 


\begin{tabular}{|c|c|c|c|c|c|c|c|c|c|c|c|c|c|}
\hline & RM 4 & 1992 & & & GENERAT & OR ANNUAL DANGEROUS WASTE & EPORT & & 994 & & $\overline{\mathrm{RM}}$ & & \\
\hline & R EPA/STATE I.D. & & $\begin{array}{l}\text { 13. } \\
\text { RECI } \\
\text { Sea } \\
\text { EPA/STAT: }\end{array}$ & $\begin{array}{l}\text { PIVING FACIL } \\
\text { Page B } \\
\text { EE I.D. NUMBE }\end{array}$ & $\begin{array}{ll}\text { LITY (TSD) } & \text { MAM } \\
& \text { ADD } \\
\text { ER } & \text { CII }\end{array}$ & 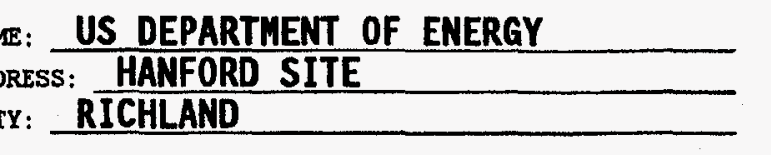 & $\begin{array}{l}\text { 14. TRANSPORTER } \\
\text { Soe Page } 8 \\
\text { EPA/STATE T.D. NMMBER }\end{array}$ & $\begin{array}{l}\text { MAME: } \\
\text { ADDRESS: } \\
\text { CITY: }\end{array}$ & 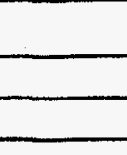 & 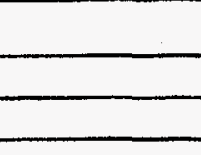 & & {[} & \\
\hline$w$ & \begin{tabular}{l|l|l|l|l|l|l|}
7 & 8 & 9 & 0 & 0
\end{tabular} & \begin{tabular}{l|l|l|l|}
8 & 9 & 6 & 7
\end{tabular} & $\mathbf{A}$ & \begin{tabular}{l|l|l|l}
7 & 8 & 9 & 0 \\
\end{tabular} & \begin{tabular}{ll|l|l|l}
0 & 0 & 0 & 8 & 9
\end{tabular} & 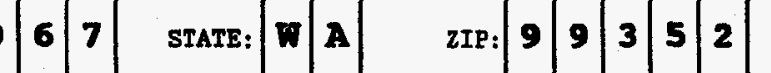 & & & STATE: & zIP: & & & \\
\hline 25. & E IDENTIEICATION & & c. $s$ & & & & & & в. & I. & s. & & \\
\hline \begin{tabular}{|l}
2 \\
$I$ \\
N \\
E
\end{tabular} & $\begin{array}{c}\text { M. } \\
\substack{\text { Manifest } \\
\text { Document } \\
\text { Number }} \\
\text { Són Page } 9\end{array}$ & 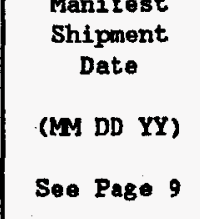 & $\mid$\begin{tabular}{r|}
$t$ \\
$\mathrm{a}$ \\
$\mathrm{t}$ \\
$\mathrm{u}$ \\
$\mathrm{s}$ \\
$\mathrm{sea}$ \\
$\mathrm{p.g}$
\end{tabular} & \begin{tabular}{|l|} 
PState \\
State \\
S=solid \\
L=Liquid \\
G=Sludge \\
M-Compresse \\
Soe Page 10
\end{tabular} & $\begin{array}{l}\text { Nature } \\
\text { O=Organ1c } \\
\text { I=Inorganio } \\
\text { d Gas } \\
\text { See Page 10 }\end{array}$ & $\begin{array}{l}\text { Waste Description } \\
\text { See Page } 10\end{array}$ & & $\begin{array}{c}\text { Dangerous Waste } \\
\text { Number } \\
\text { (see instructions) } \\
\text { and WAC 173-303) } \\
\text { See Page 11 }\end{array}$ & 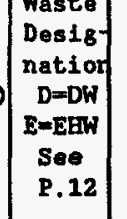 & $\begin{array}{c}\text { Weignt } \\
\text { of } \\
\text { Wasto } \\
\text { Soe Page } 12\end{array}$ & $\begin{array}{ll}E & c \\
E & C \\
I & 0 \\
G & D \\
B & E \\
T \\
P .12\end{array}$ & 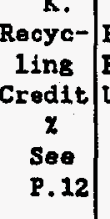 & \begin{tabular}{|l} 
For TsD \\
Facility \\
Wuse OnI \\
Soe. \\
P.13
\end{tabular} \\
\hline 42 & 24502 & 071294 & & $\mathbf{s}$ & Io & 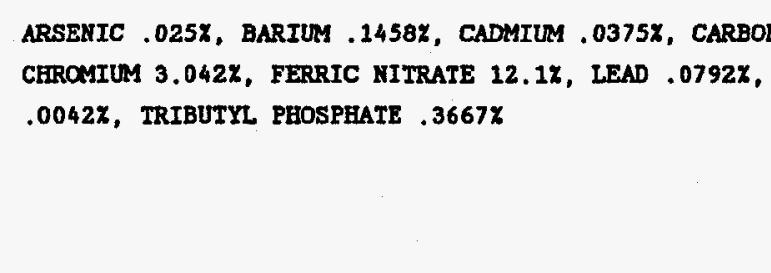 & $\begin{array}{l}\text { ETRACHLORIDE . 5083\%, } \\
\text { LENIUM .0042X, SILVER }\end{array}$ & $\begin{array}{l}\text { D004 D005 } \\
\text { D006 D007 } \\
\text { D008 D010 } \\
\text { D011 D019 } \\
\text { WC02 WP01 } \\
\text { WT02 }\end{array}$ & RE & 24 & $\mathrm{x}$ & & \\
\hline 42 & 24607 & 080994 & & $\mathbf{s}$ & Io & CEROMIUM . 03562 & & D007 wC02 & RD & эз & $\mathrm{x}$ & & \\
\hline 43 & 24607 & 080994 & &.$^{s}$ & Io & CADMTUM , 0691X, CHROMIUM .0025X & & $\begin{array}{l}\text { D006 D007 } \\
\text { WC02 }\end{array}$ & $\mathrm{RD}$ & 17 & $\mathrm{x}$ & & \\
\hline 44 & 24607 & 080994 & & $\mathbf{s}$ & Io & ARSENIC .0122Z, CADMIUM ,0057X, CAROMIUM .1569z & & $\begin{array}{l}\text { D004 D006 } \\
\text { D007 WOC2 }\end{array}$ & RD & 30 & $\mathrm{k}$ & & \\
\hline 45 & 24607 & 080994 & & s & Io & CADMIUM $.0026 \mathrm{X}$, CRROMIUM $.0694 \mathrm{X}$ & & $\begin{array}{l}\text { D006 D007 } \\
\text { WC02 }\end{array}$ & RD & 62 & $\mathrm{x}$ & & \\
\hline 46 & 24651 & 091994 & & L & Io & SODIUM NITRATE $1.429 x$ & & wro2 & RD & 14 & $\mathrm{x}$ & & \\
\hline & $\begin{array}{l}\text { s (Enter in } \\
\text { Y: }\end{array}$ & by $\mathbf{s e}$ & & numbe & Page 1 & & & & & & & & \\
\hline & & & & & & & & & & PAGE 4 & 16 & & \\
\hline
\end{tabular}




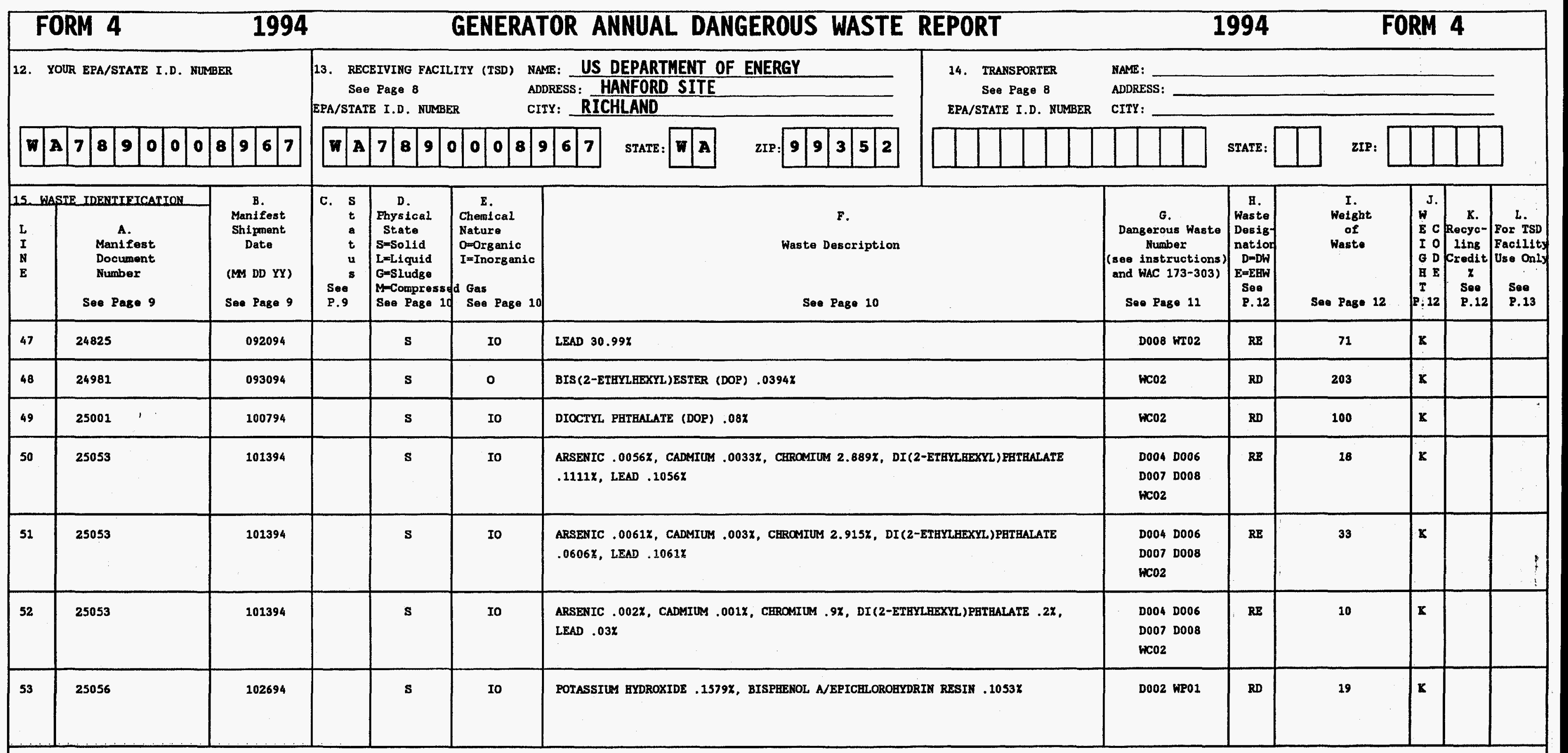

16. COMENTS (Enter information by section and/or line number--Soe Page 13). FACILITY: 2345Z

PAGE 49 OF 161 


\begin{tabular}{|c|c|c|c|c|c|c|c|c|c|c|c|c|c|}
\hline \multicolumn{2}{|c|}{ FORM 4} & 1994 & \multicolumn{5}{|c|}{ GENERATOR ANNUAL DANGEROUS WASTE REPORT } & & 994 & \multicolumn{4}{|c|}{ FORM 4} \\
\hline \multicolumn{3}{|c|}{ 12. YOUR EPA/STATE T.D. MUMBER } & \multicolumn{4}{|c|}{ 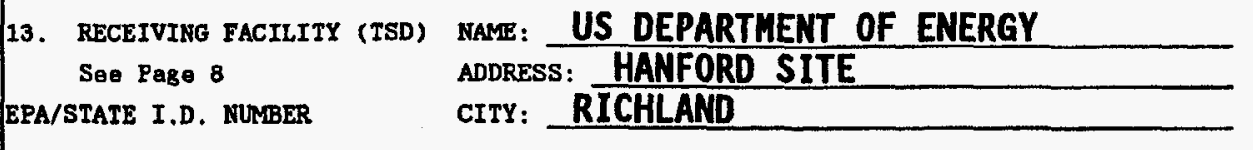 } & $\begin{array}{l}\text { 14. IRANSPORTIR } \\
\text { See Page } 8 \\
\text { EPA/STATE I.D. NUMBER }\end{array}$ & \multicolumn{5}{|l|}{$\begin{array}{l}\text { NAME: } \\
\text { ADDRESS: } \\
\text { CITY: }\end{array}$} & \\
\hline $\mathbf{w}$ & \begin{tabular}{|l|l|l|l|l|}
7 & 8 & 9 & 0 & 0 \\
\end{tabular} & \begin{tabular}{|l|l|l|l|}
8 & 9 & 6 & 7 \\
\end{tabular} & 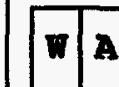 & \begin{tabular}{|l|l|l|l}
7 & 8 & 9 & \\
\end{tabular} & \begin{tabular}{l|l|l|l|l}
0 & 0 & 0 & 8 & 9
\end{tabular} & 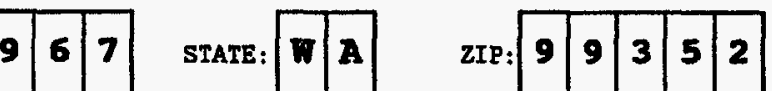 & & & STATE: & zIP: & & & \\
\hline \multicolumn{2}{|c|}{ 15_ hASTE IDERTIETCATTOK } & \multirow{2}{*}{$\begin{array}{c}\text { B. } \\
\text { Manifest } \\
\text { Shipment } \\
\text { Date } \\
\text { (MM DD YY) } \\
\text { Sea Page } 9\end{array}$} & \multirow{2}{*}{$\begin{array}{l}\text { c. } s \\
t \\
t \\
t \\
u \\
s \\
\text { Sea } \\
\text { P.9 }\end{array}$} & \multirow{2}{*}{ 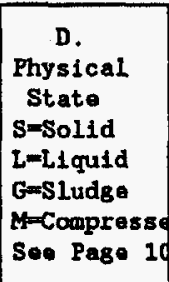 } & \multirow{2}{*}{\begin{tabular}{|c|}
\multicolumn{1}{|c|}{} \\
Chemical \\
Nature \\
O=Organic \\
I-Inorganic \\
d Gas \\
Soo Page 10
\end{tabular}} & \multirow{2}{*}{\multicolumn{2}{|c|}{$\begin{array}{c}F . \\
\text { Waste Description }\end{array}$}} & \multirow[b]{2}{*}{$\begin{array}{l}\text { G. } \\
\text { Dangorous waste } \\
\text { Number } \\
\text { (soo instructions) } \\
\text { and WAC 173-303) } \\
\text { Soe Page } 11\end{array}$} & \multirow{2}{*}{ 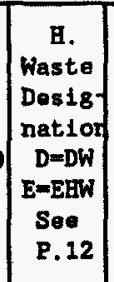 } & \multirow{2}{*}{$\begin{array}{c}\begin{array}{c}\text { I. } \\
\text { Waight } \\
\text { of } \\
\text { Wasto }\end{array} \\
\text { Soe Page } 12\end{array}$} & \multirow{2}{*}{ 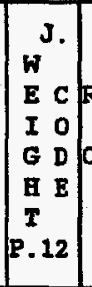 } & \multirow[b]{2}{*}{ 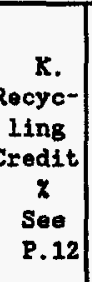 } & \multirow[b]{2}{*}{ 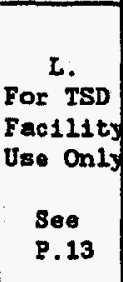 } \\
\hline $\begin{array}{l}\mathrm{L} \\
\mathbf{L} \\
\mathrm{N} \\
\mathrm{E}\end{array}$ & $\begin{array}{l}\text { Manif fest } \\
\text { Documont } \\
\text { Number } \\
\text { Sol Page } 9\end{array}$ & & & & & & & & & & & & \\
\hline 54 & 25131 & 102694 & & s & Io & LEAD .0833X, XYLENE .0333X & & $\begin{array}{l}\text { DOOB HCO2 } \\
\text { WTO1 }\end{array}$ & $\mathrm{RE}$ & 60 & $\mathbf{k}$ & & \\
\hline \multicolumn{14}{|c|}{$\begin{array}{l}\text { 16. COMMENTS (Enter information by soction and/or line number--See Page i3). } \\
\text { PACCLITY: } 23452\end{array}$} \\
\hline & & & & & & & & & & $\begin{array}{ll}\text { PAGE } 50 \\
\end{array}$ & F 161 & & \\
\hline
\end{tabular}




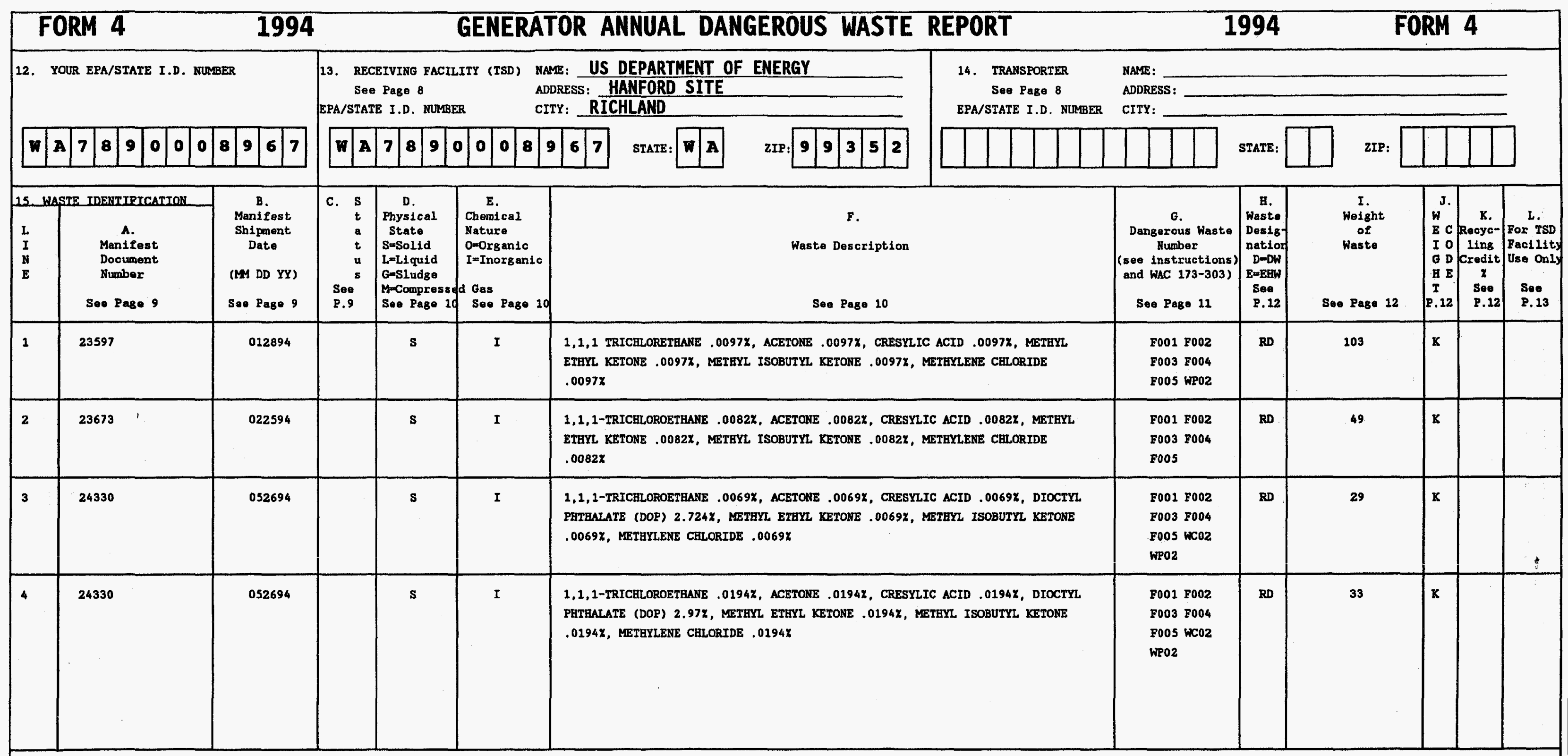

16. congrests (Enter information by section and/or line number--See Page 13). FACILITY: 241A 


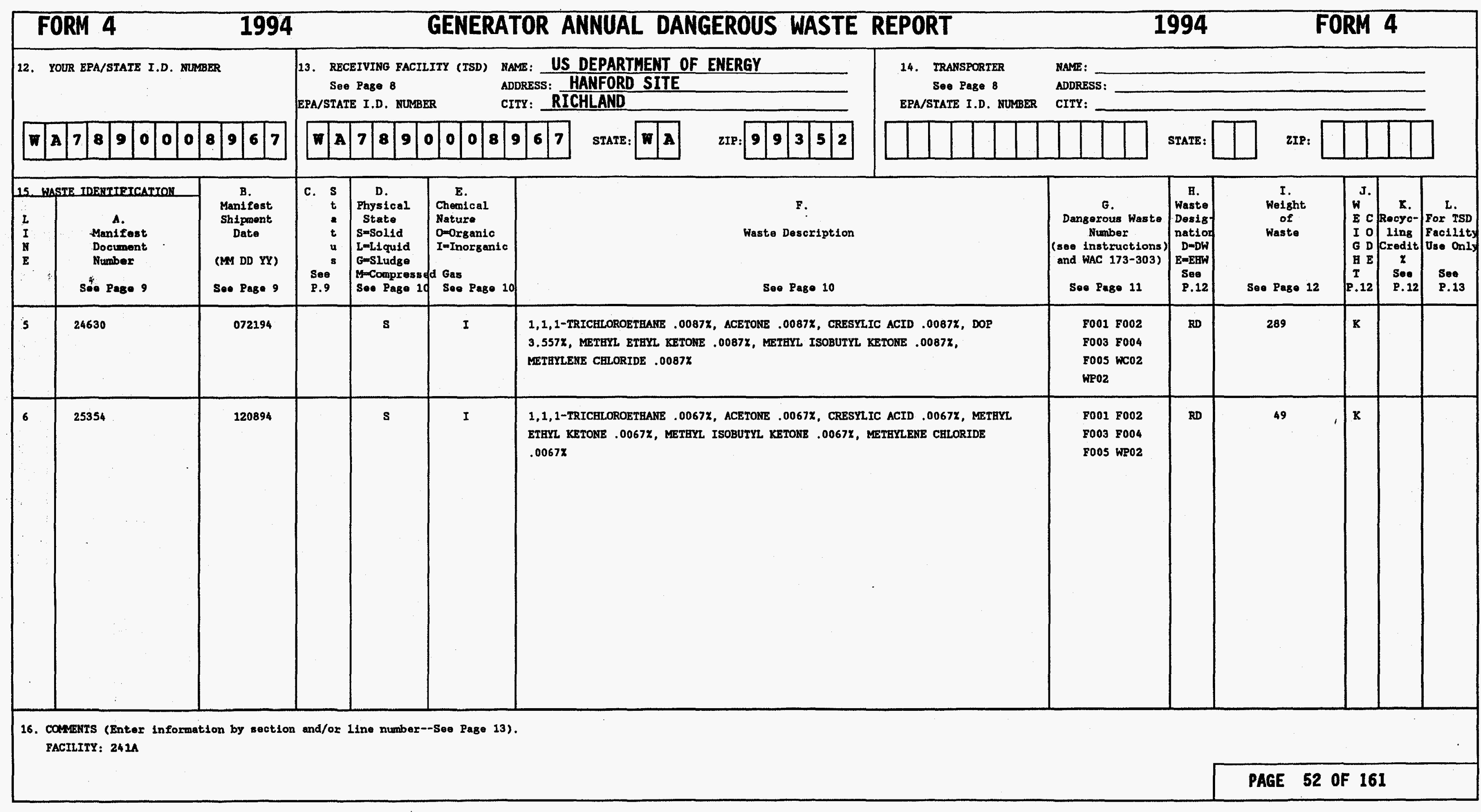




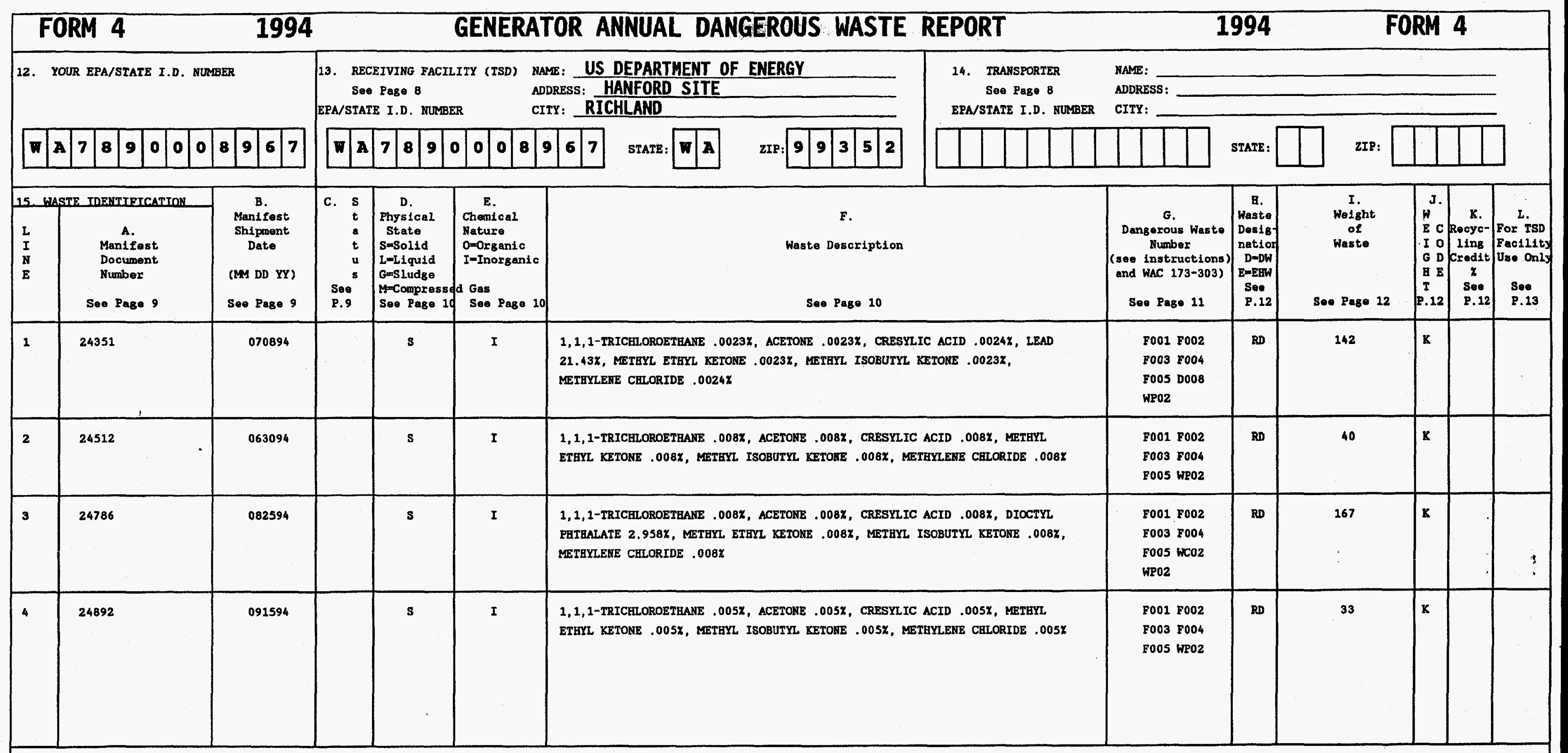

16. Coments (Enter information by section and/or line number--See Page 13). FACILITY: 241AN 


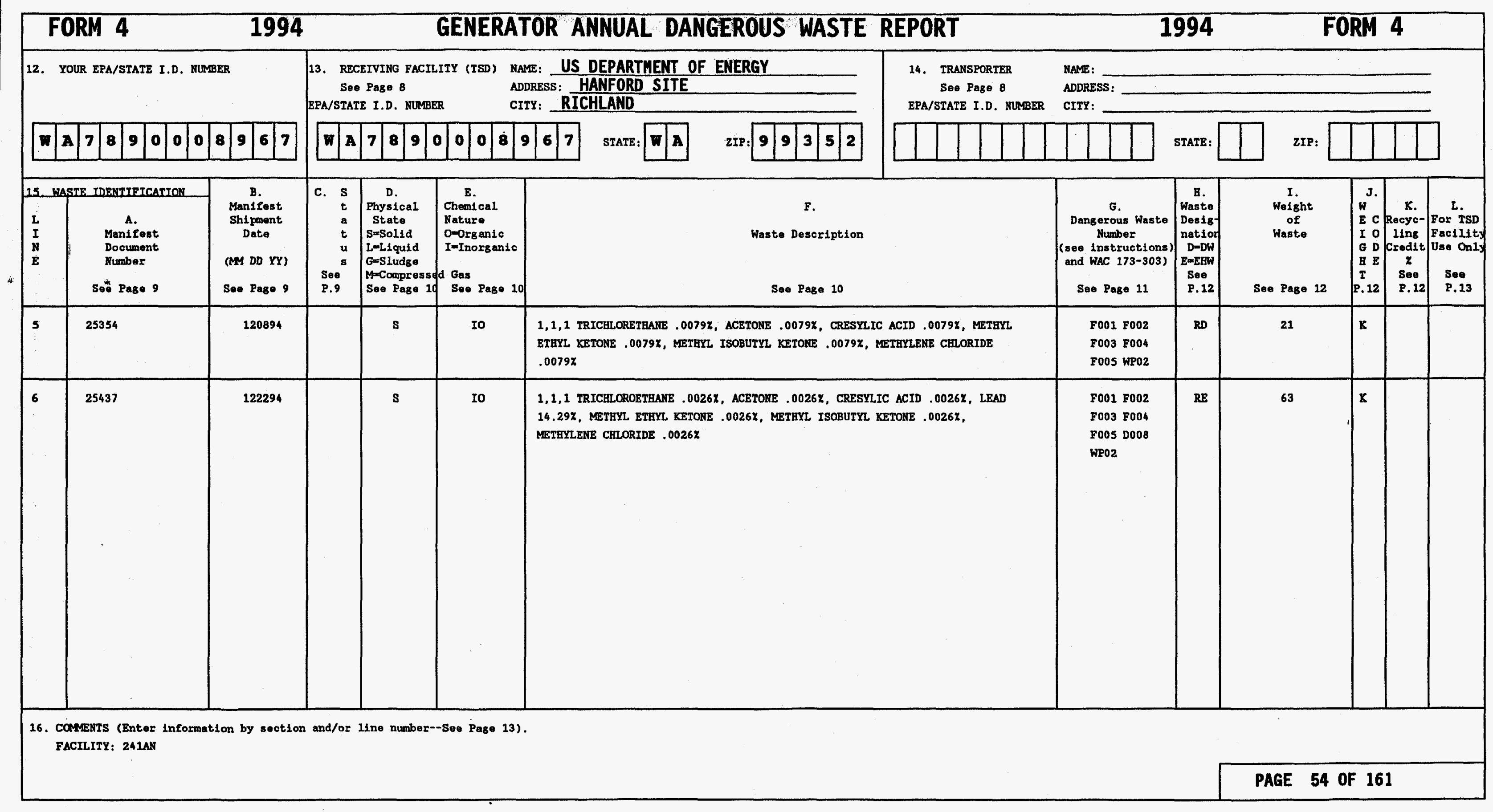




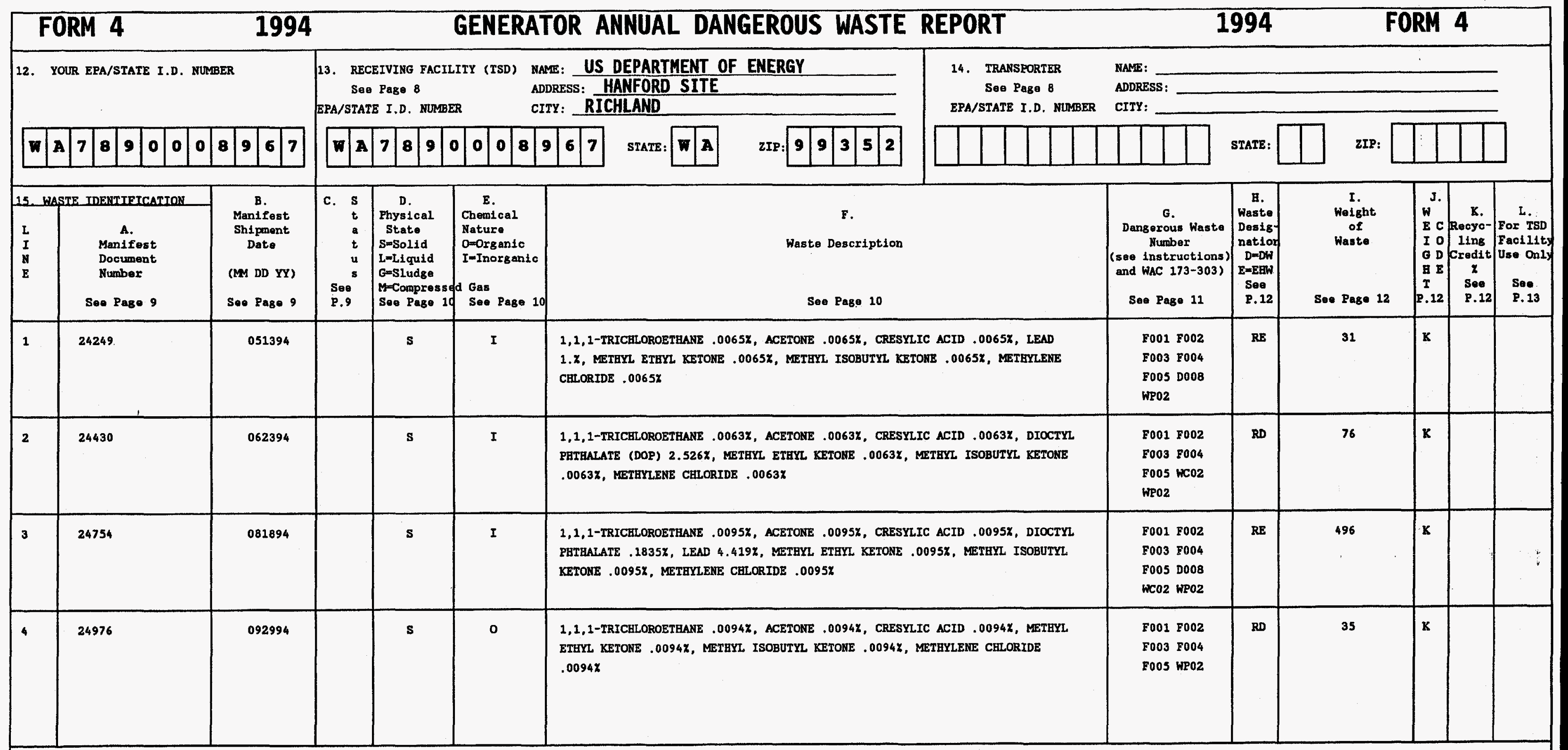

16. Converss (Enter information by soction and/or line number--See Page 13). FACILITY: $241 \mathrm{AP}$

PAGE 55 OF 161 


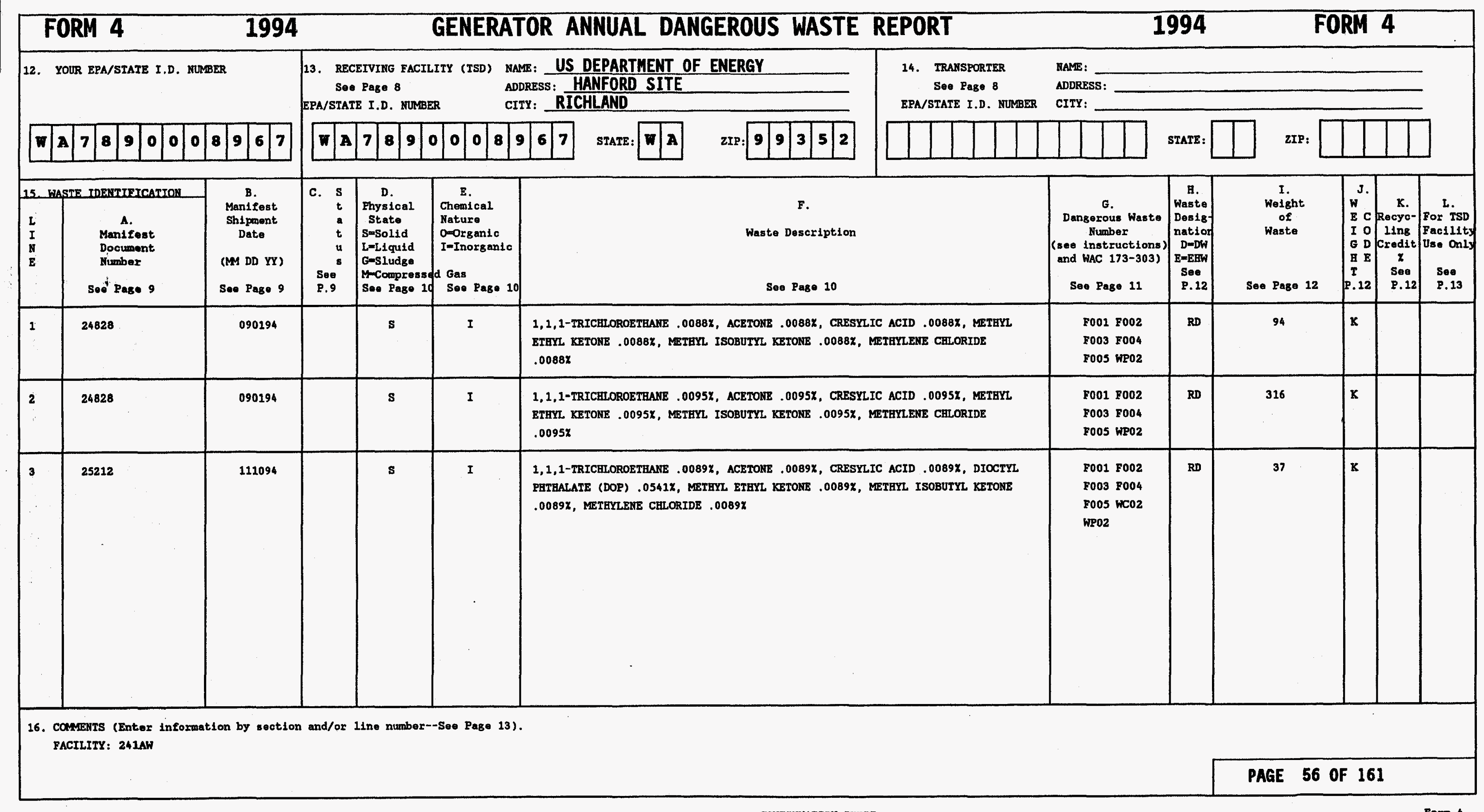




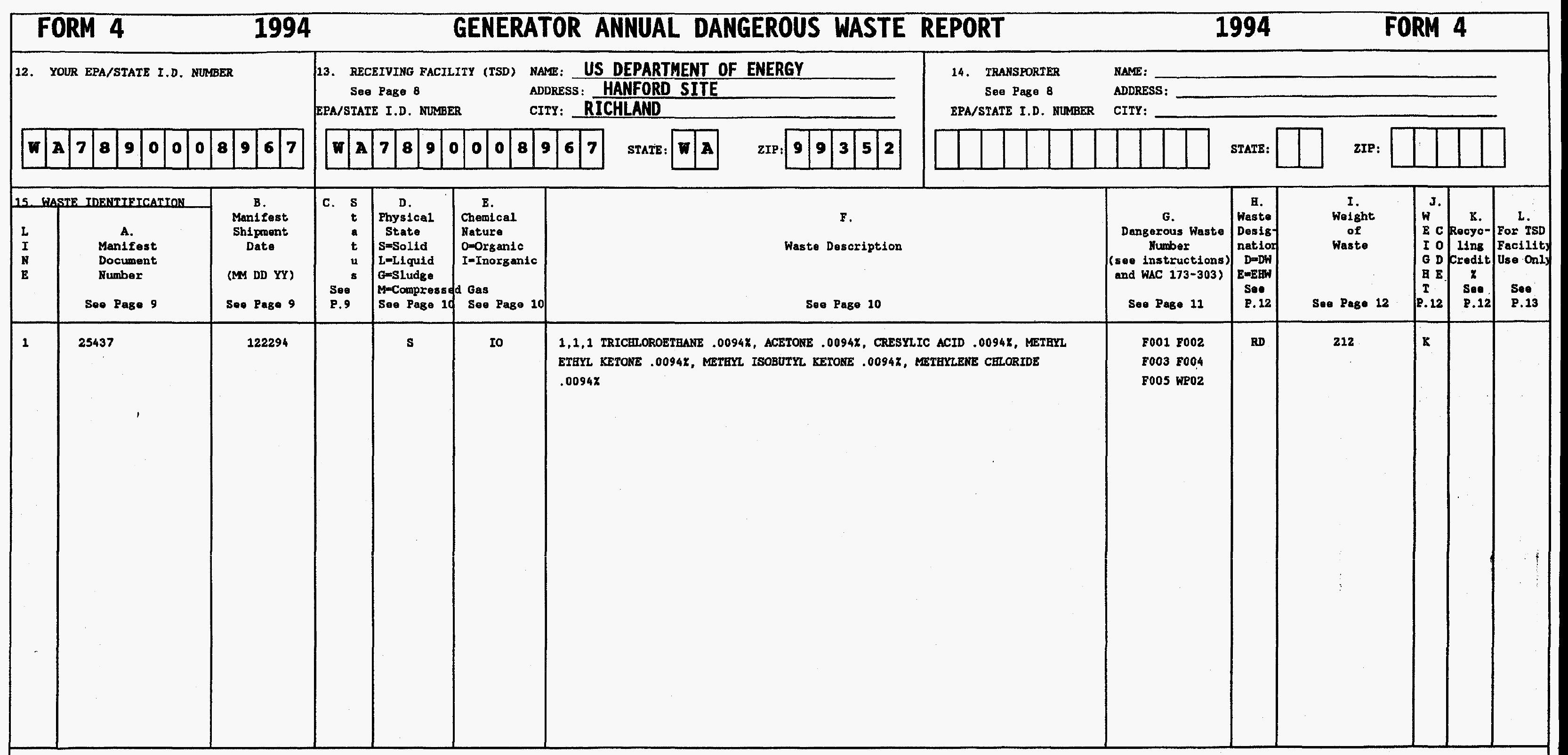

16. COMENTS (Enter information by section and/or line number--See Page 13). FACILITY: $241 \mathrm{AX}$ 


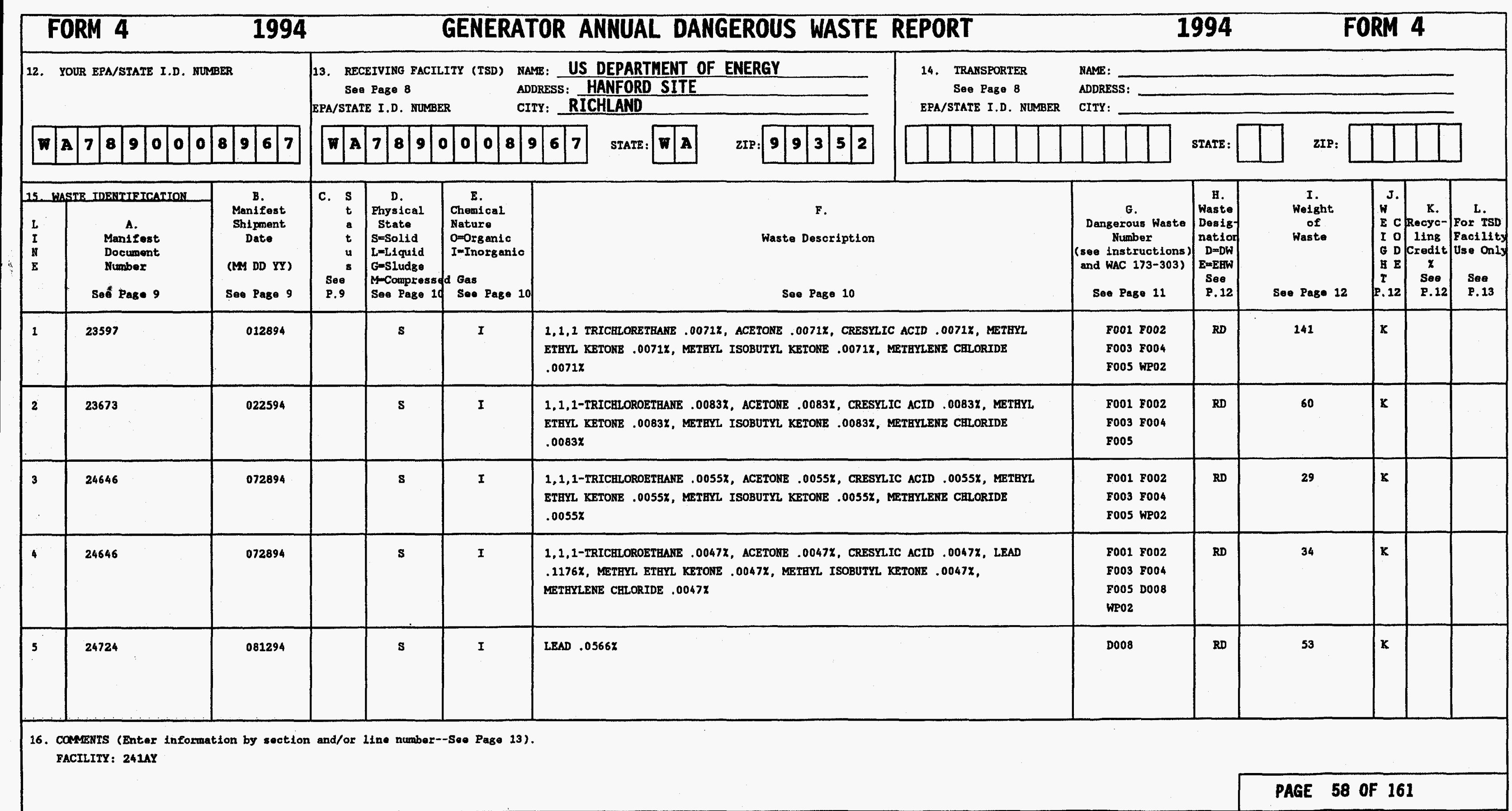




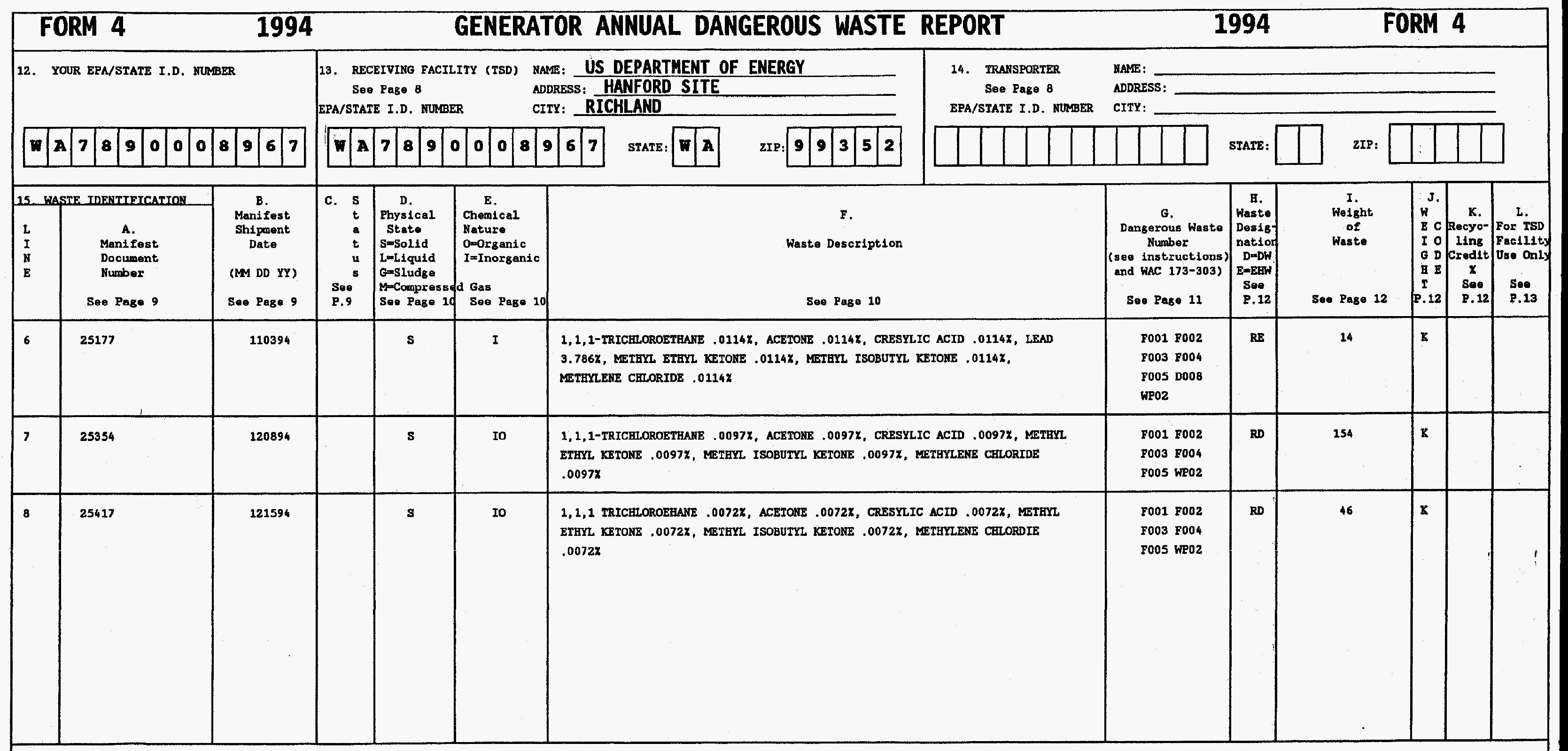

16. COMveNTS (Enter information by section and/or line number--See Page 13). FACILITY: 2AIAY 


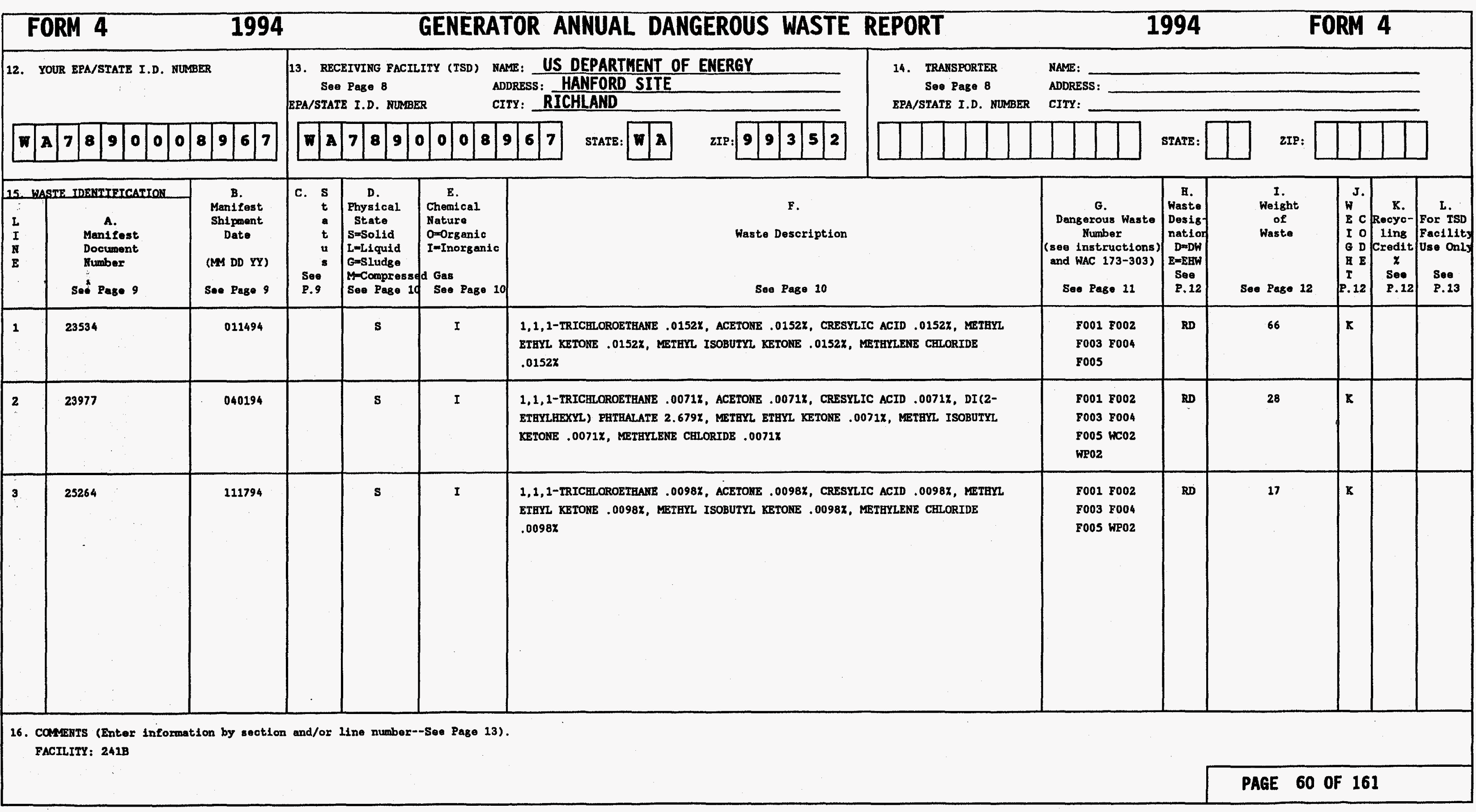




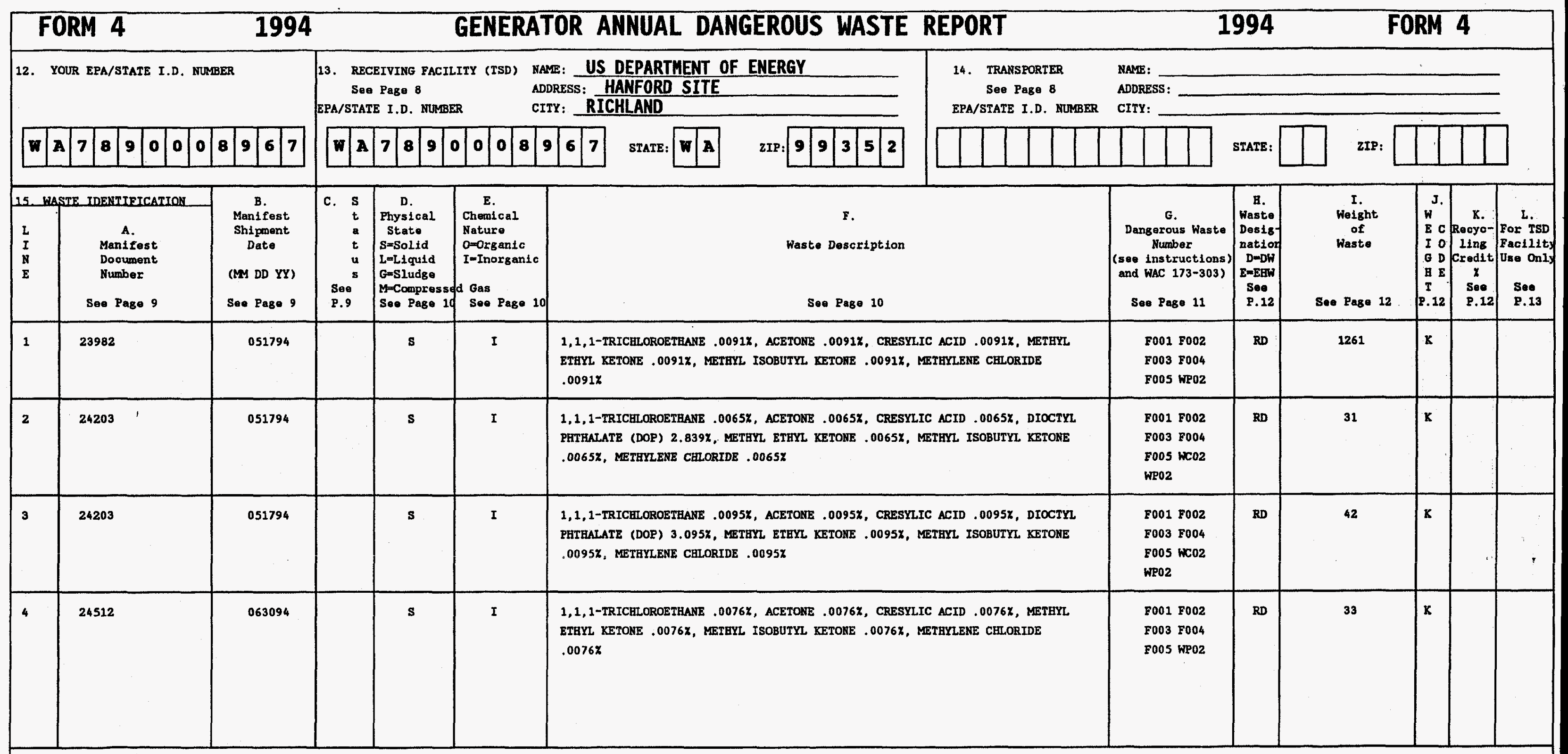

16. COMENTS (Enter information by soction and/or line number--Soe Page 13). FACILITY: 241BX

PAGE 61 OF 161 


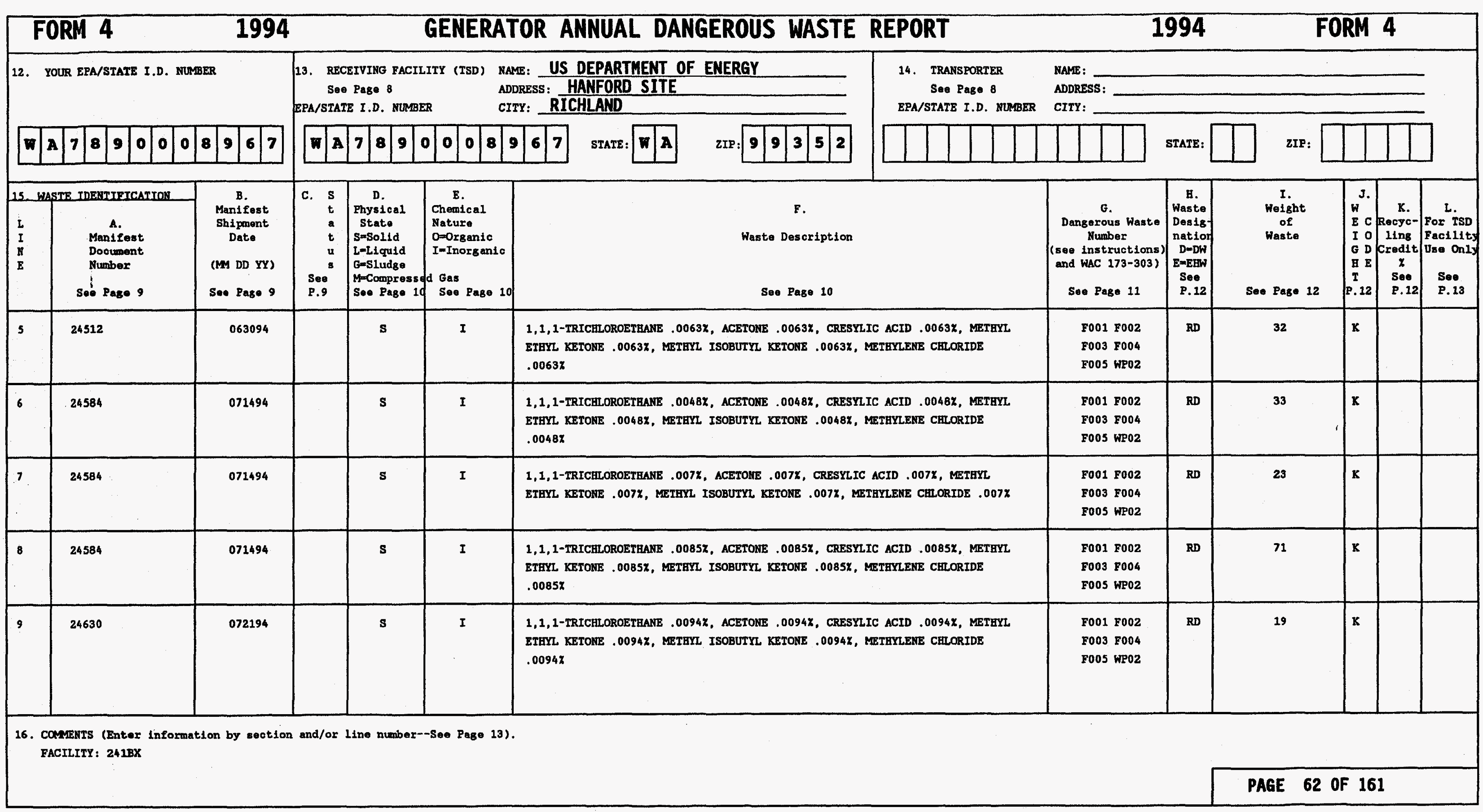




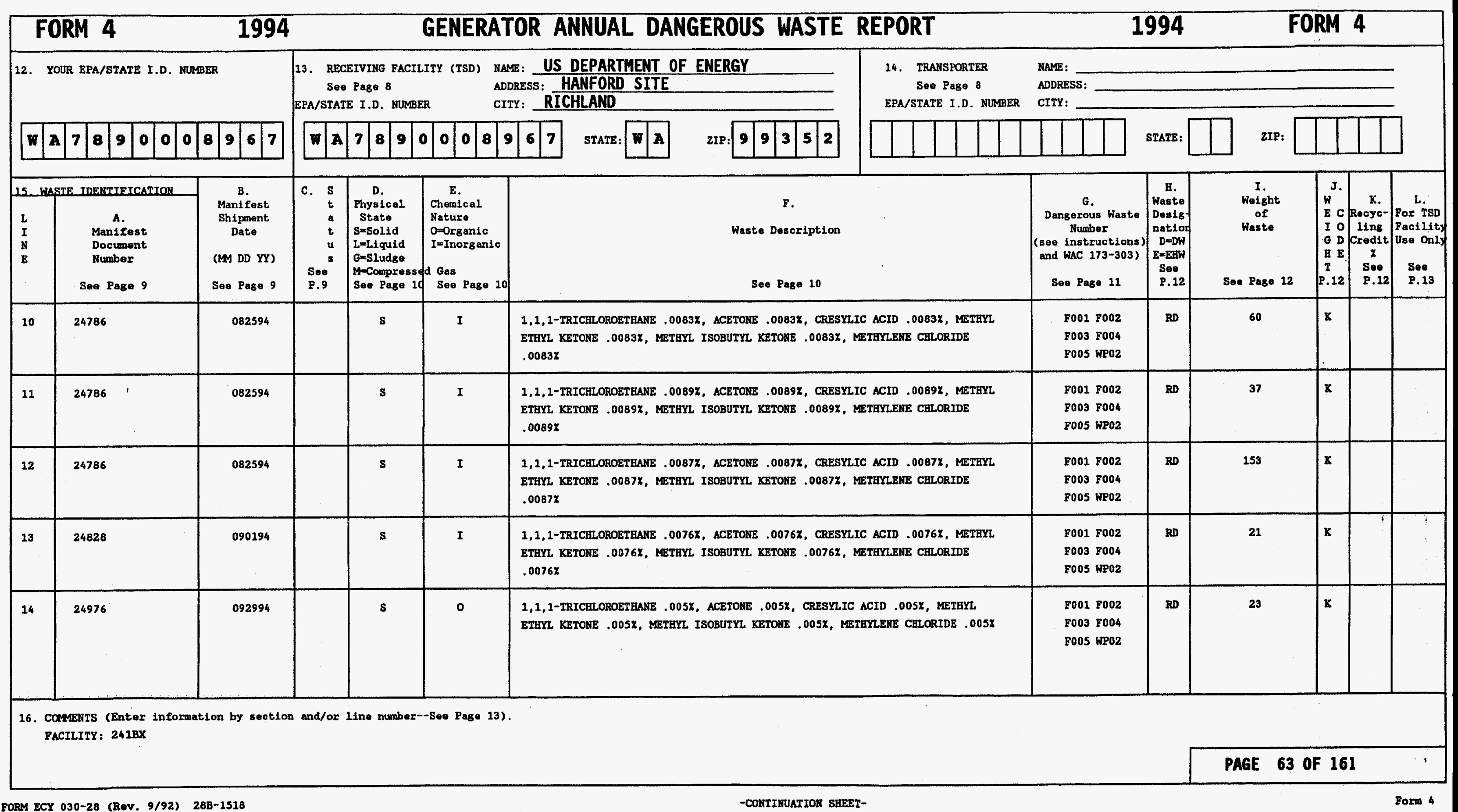




\begin{tabular}{|c|c|c|c|c|c|c|c|c|c|c|c|c|c|}
\hline & RM 4 & 199 & & & GENERAT & OR ANNUAL DANGEROUS WASTE & EPORT & & 994 & & $\mathbf{R M}$ & & \\
\hline & UR EPA/STATE I.D. N & & $\begin{aligned} & 13 . \text { REC } \\
& \text { See } \\
& \text { SPA/STAT }\end{aligned}$ & $\begin{array}{l}\text { EEIVING FACII } \\
\text { Page } 8 \\
\text { IE I.D. NUMBE }\end{array}$ & $\begin{array}{ll}\text { LITY (ISD) } & \text { NAM } \\
& \text { ADD } \\
\text { ER } & \text { CIT }\end{array}$ & $\begin{array}{l}\text { E: } \frac{\text { US DEPARTMENT OF ENERGY }}{\text { RESS: }} \text { HANFORD SITE } \\
\text { Y: RICHLAND }\end{array}$ & $\begin{array}{l}\text { 14. IRAMSPORTER } \\
\text { Soe Page } 8 \\
\text { EPA/STAIE I.D. MUMBER }\end{array}$ & $\begin{array}{l}\text { NAME: } \\
\text { ADDRESS: } \\
\text { CITY: }\end{array}$ & & & & & \\
\hline $\mathbf{w}$ & \begin{tabular}{|l|l|l|l|l|l|l|}
7 & 8 & 9 & 0 & 0 & 0 \\
\end{tabular} & \begin{tabular}{l|l|l|l|}
8 & 9 & 6 & 7 \\
\end{tabular} & \begin{tabular}{|l|l}
$\pi$ & A \\
\end{tabular} & \begin{tabular}{|l|l|l|l}
7 & 8 & 9 & 1 \\
\end{tabular} & \begin{tabular}{l|l|l|l|l|}
0 & 0 & 0 & 8 & 9 \\
\end{tabular} & 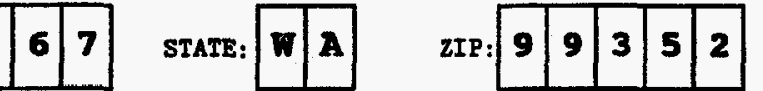 & & & STATE: & 2IP: & & & \\
\hline 25. & IE. IDENTIEICATTOON & & c. $s$ & & & & & & 0 & I. & J. & & \\
\hline $\begin{array}{l}\mathrm{L} \\
\mathrm{I} \\
\mathrm{N} \\
\mathrm{E}\end{array}$ & $\begin{array}{l}\text { A. } \\
\text { Manifest } \\
\text { Dooument } \\
\text { Number } \\
\text { Sul Page } 9\end{array}$ & $\begin{array}{c}\begin{array}{c}\text { Shypment } \\
\text { Date }\end{array} \\
\text { (IM DD YY) } \\
\text { Seo Page } 9\end{array}$ & $\begin{array}{c}\mathrm{a} \\
\mathrm{t} \\
\mathrm{u} \\
\mathrm{s} \\
\text { So: } \\
\mathrm{P.9}\end{array}$ & $\begin{array}{l}\text { State } \\
\text { S=Solid } \\
\text { L=Liquid } \\
\text { G=Sludge } \\
\text { M-Compress } \\
\text { Seo Page 10 }\end{array}$ & $\begin{array}{l}\text { Nature } \\
\text { O=-Organic } \\
\text { I=Inorganic } \\
\text { da Gas } \\
\text { Seo Page } 10\end{array}$ & $\begin{array}{l}\text { Waste Description } \\
\text { Seo Page } 10\end{array}$ & & $\begin{array}{c}\text { Dangerous Waste } \\
\text { Number } \\
\text { (see instructions) } \\
\text { and WAC 173-303) } \\
\text { Seo Page } 11\end{array}$ & 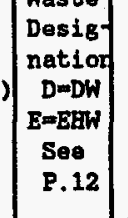 & See Page 12 & $\mid \begin{array}{lll}E & C \\
I & 0 \\
G & D \\
a & E \\
I \\
P .12\end{array}$ & 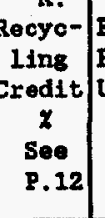 & $\begin{array}{l}\text { For isp } \\
\text { Facilits } \\
\text { use onls } \\
\text { soe } \\
\text { P.13 }\end{array}$ \\
\hline 15 & 24998 & 100694 & & $\mathbf{s}$ & I & $\begin{array}{l}\text { 1,1,1-TRTCHLOROETBANE .0094Z, ACETONE .0094X, CRESY } \\
\text { ETHY KETONE .0094X, METHY ISOBUTY KETONE .0094X, } \\
.0094 Z\end{array}$ & $\begin{array}{l}\text { ACID .0094\%, METHYY } \\
\text { EYLENE CHLORIDE }\end{array}$ & $\begin{array}{l}\text { F001 F002 } \\
\text { F003 F004 } \\
\text { F005 WR02 }\end{array}$ & RD & 53 & $\mathbf{x}$ & & \\
\hline 16 & 25177 & 110394 & & s & I & 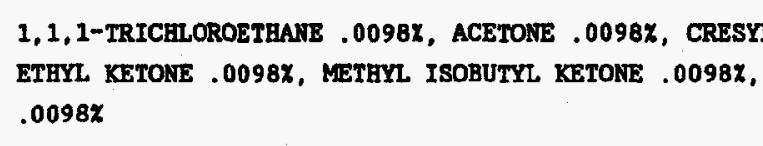 & $\begin{array}{l}\text { ACID .0098X, METEYR } \\
\text { BYLENE CHLORIDE }\end{array}$ & $\begin{array}{l}\text { F001 F002 } \\
\text { F003 F004 } \\
\text { F005 WP02 }\end{array}$ & RD & 51 & $\mathbf{x}$ & & \\
\hline 17 & 25212 & 211094 & & $\mathbf{s}$ & I & $\begin{array}{l}\text { 1,1,1-TRICHOROETEANE, .0092X, ACETONE, .0092X, CRESY } \\
\text { ETHYL KETONE .0092X, METEYL ISOBUTYL KETONE .0092X, } \\
.0092 X\end{array}$ & $\begin{array}{l}\text { ACID ,0092X, METHYL } \\
\text { AYLENE CRLORIDE }\end{array}$ & $\begin{array}{l}\text { F001 F002 } \\
\text { F003 F004 } \\
\text { F005 WP02 }\end{array}$ & RD & 18 & $\mathrm{x}$ & & \\
\hline 18 & 25212 & 111094 & & $s$ & I & 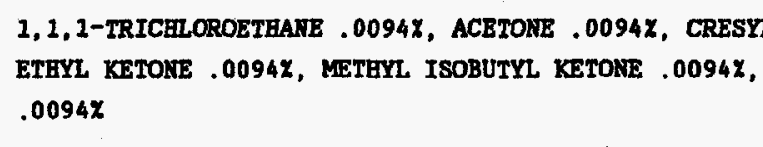 & $\begin{array}{l}\text { ACID .0094\%, METHYL } \\
\text { AYLENE CELORIDE }\end{array}$ & $\begin{array}{l}\text { F001 F002 } \\
\text { F003 F004 } \\
\text { F005 WP02 }\end{array}$ & RD & 35 & $\mathbf{x}$ & & \\
\hline 19 & 25212 & 111094 & & s & I & $\begin{array}{l}\text { 1,1,1-TRICHOOROETEANE, .0106x, ACETONE .0106x, CRESY } \\
\text { ETHYL KEYONE .0106x, METHYL ISOSUTYL KETONE .0106x, } \\
.01062\end{array}$ & $\begin{array}{l}\text { ACID .0106x, METAYI } \\
\text { BYLENE CHLORIDE }\end{array}$ & $\begin{array}{l}\text { F001 F002 } \\
\text { F003 F004 } \\
\text { F005 WF02 }\end{array}$ & RD & 16 & $\mathbf{x}$ & & \\
\hline & $\begin{array}{l}\text { (Enter } \ln f \\
2418 \mathrm{x}\end{array}$ & & & & $\mathrm{Pa}$ & & & & & & & & \\
\hline & & & & & & & & & & PAGE & 16. & & \\
\hline
\end{tabular}




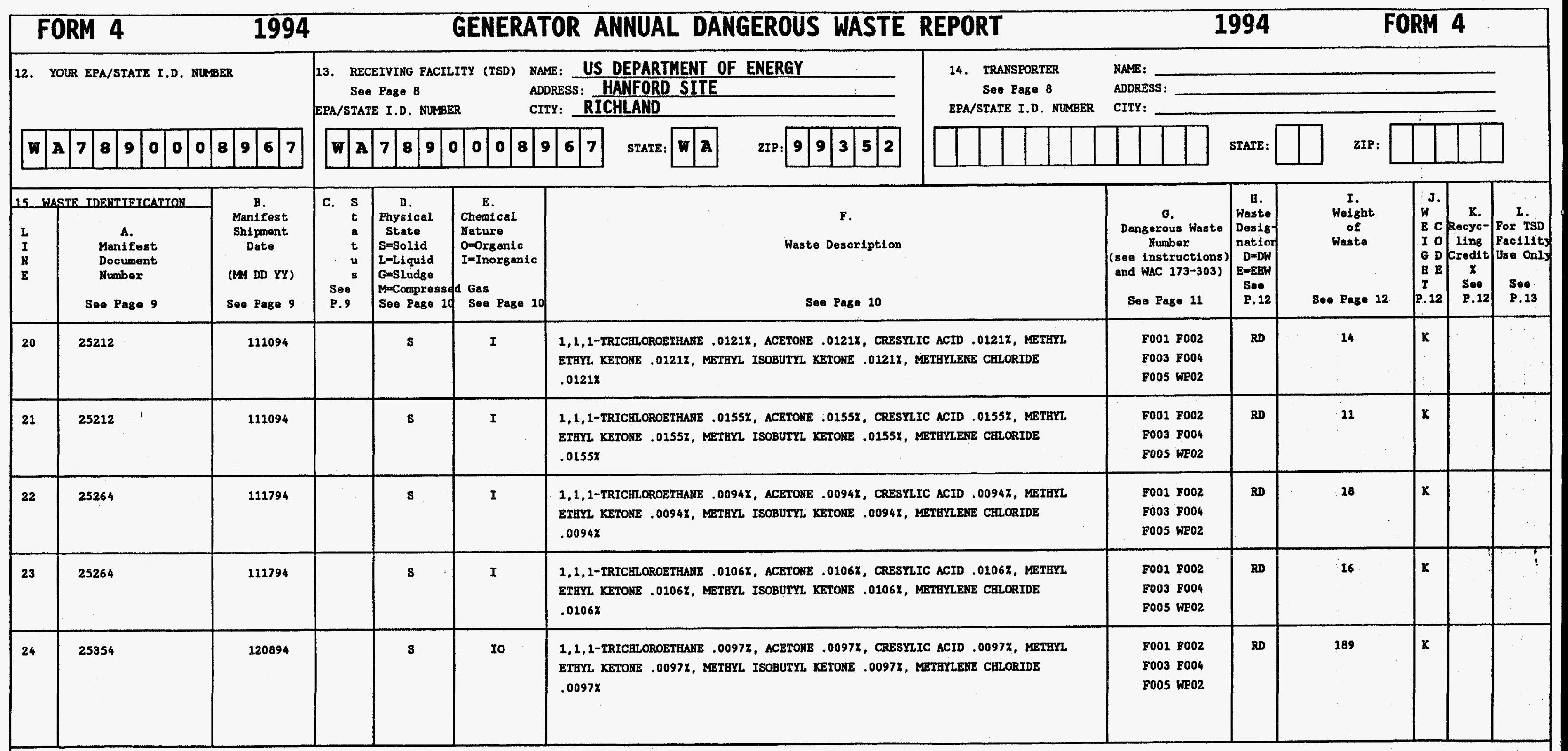

16. Compriss (Enter information by seotion and/or line number--See Page 13). FACILITY: $241 \mathrm{BX}$

PAGE 65 OF 161 


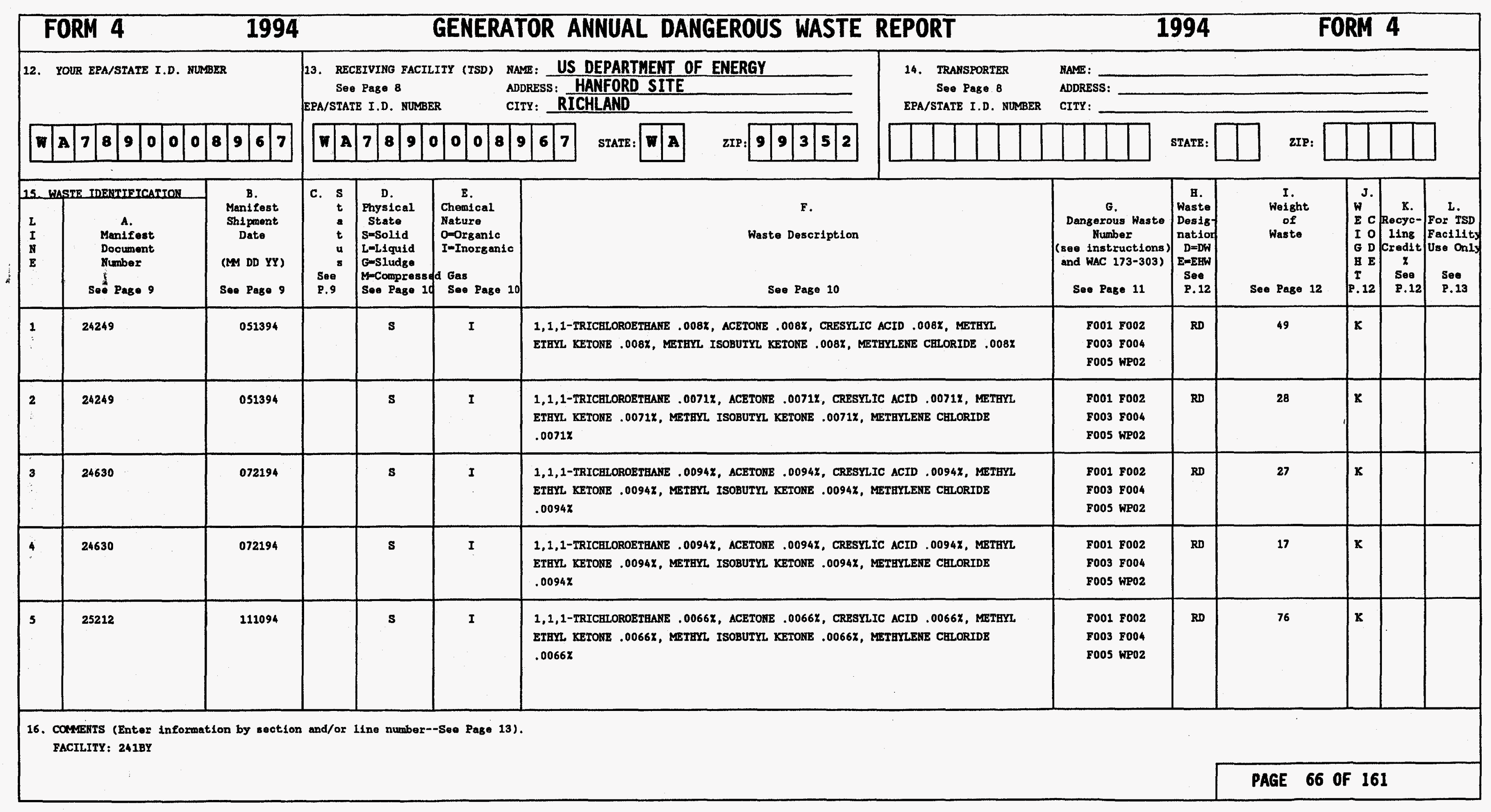




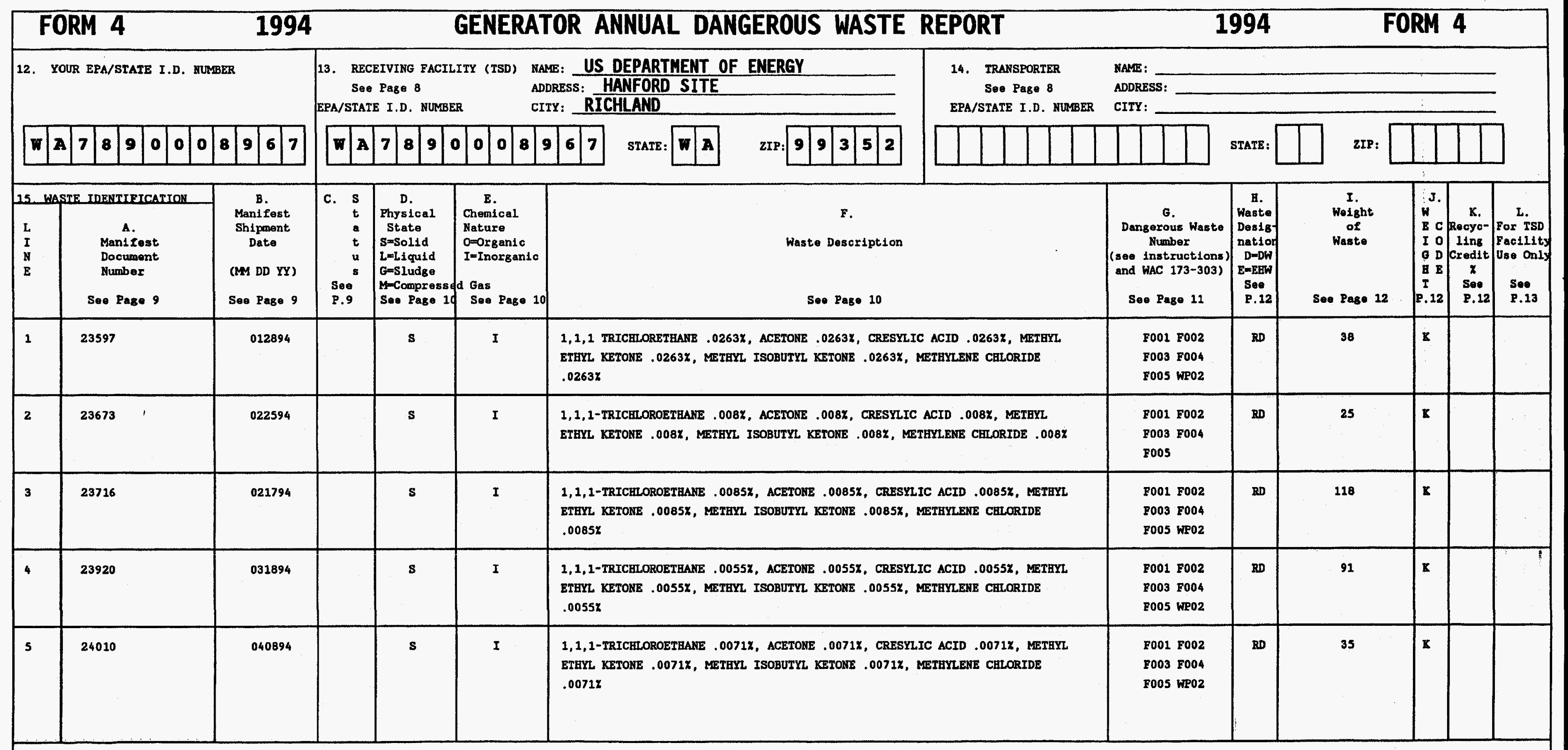

16. Comperrs (Enter information by section and/or 1ine number--See Page 13). FACILITY: $241 \mathrm{C}$

PAGE 67 OF 161 


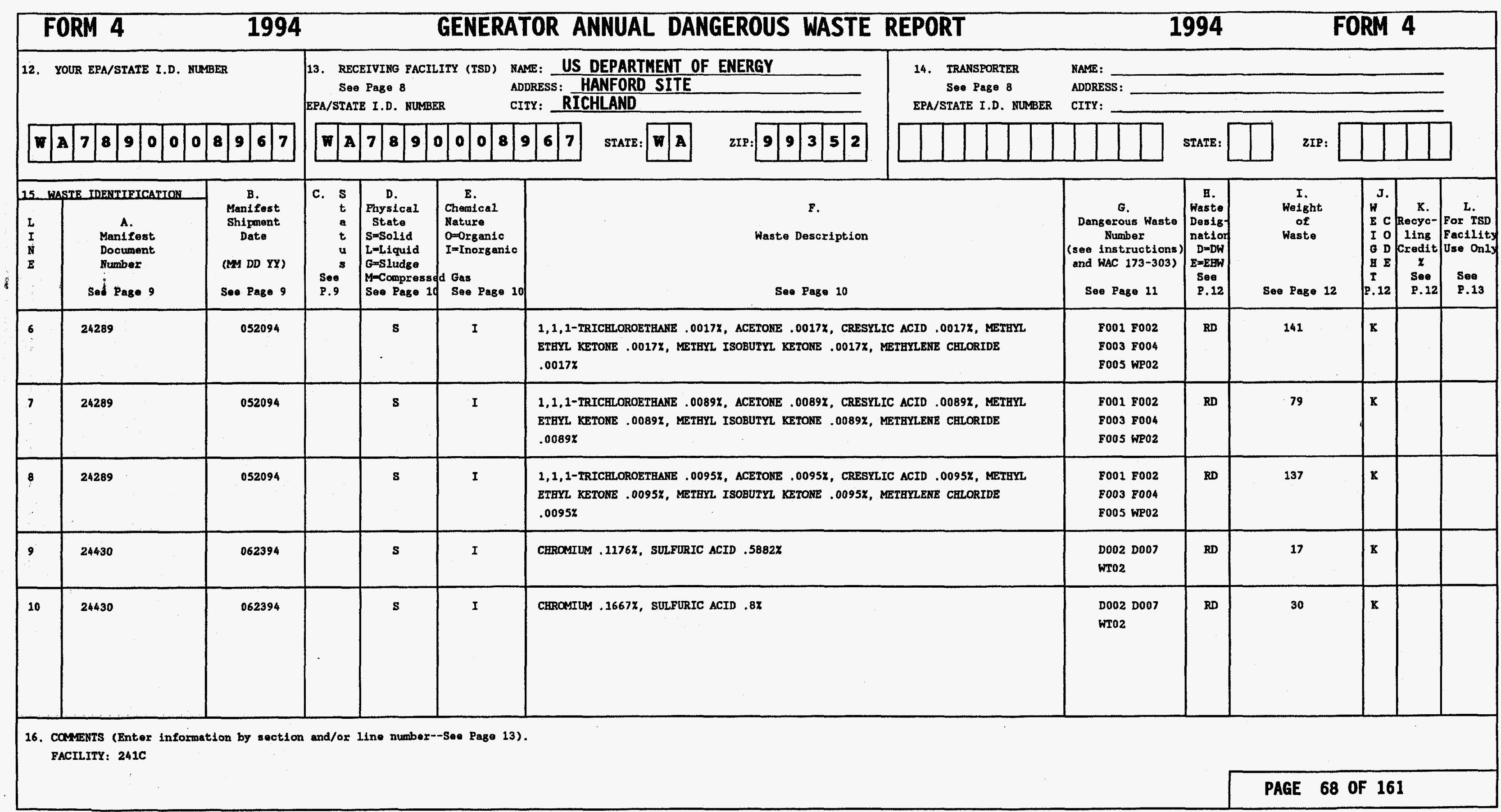




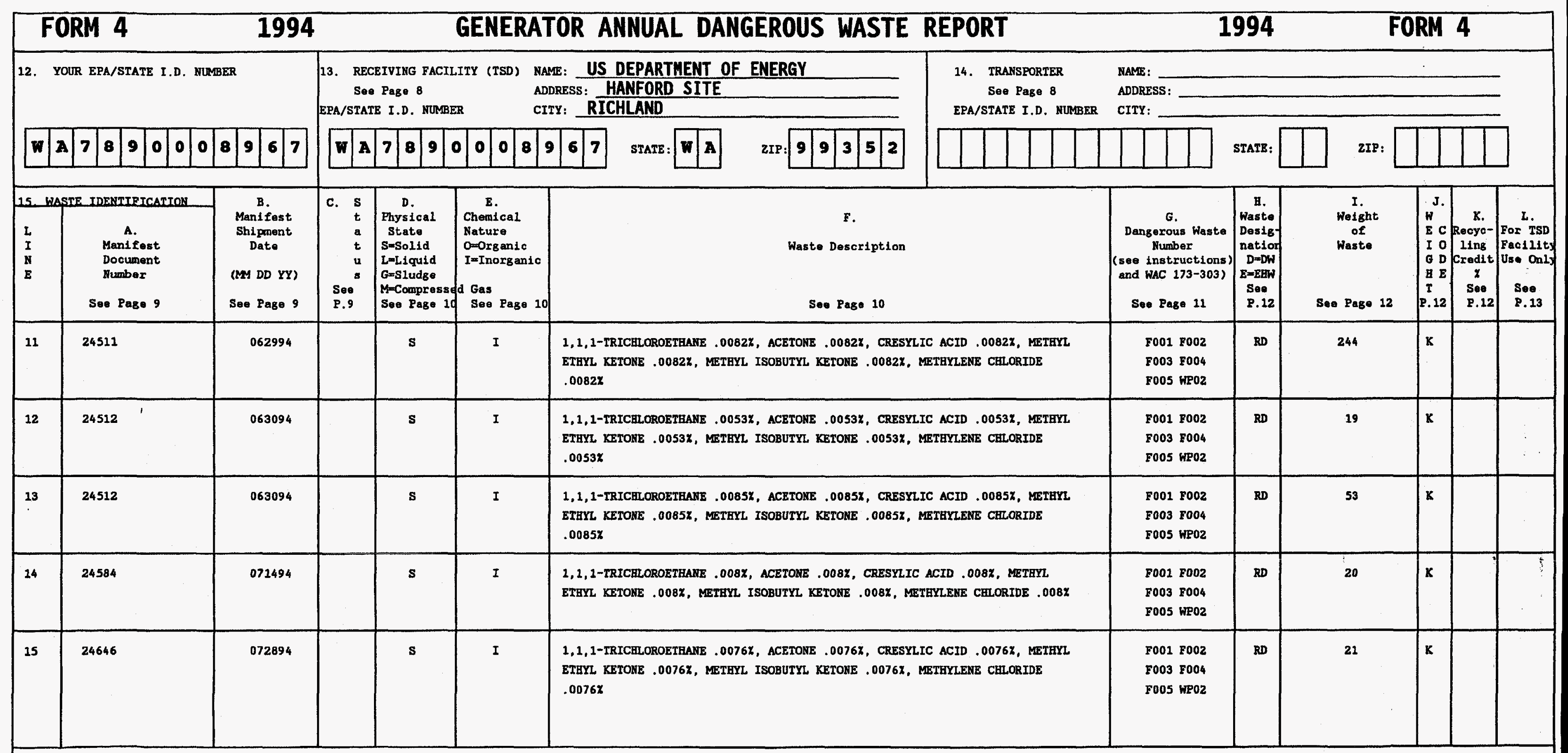

16. COMYENTS (Enter information by section and/or 1ine number--Sea Page 13). FACILITY: $241 \mathrm{C}$ 


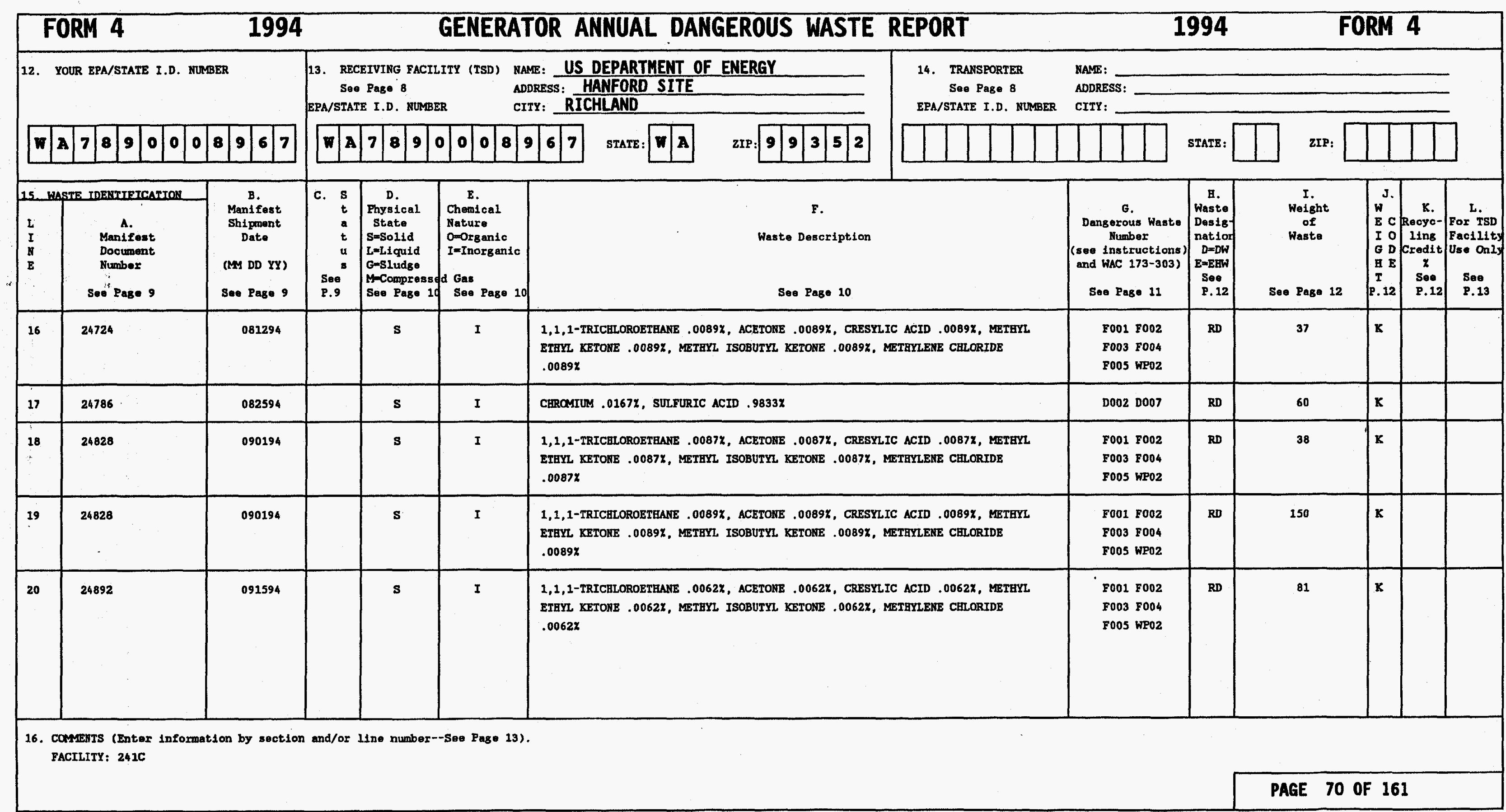




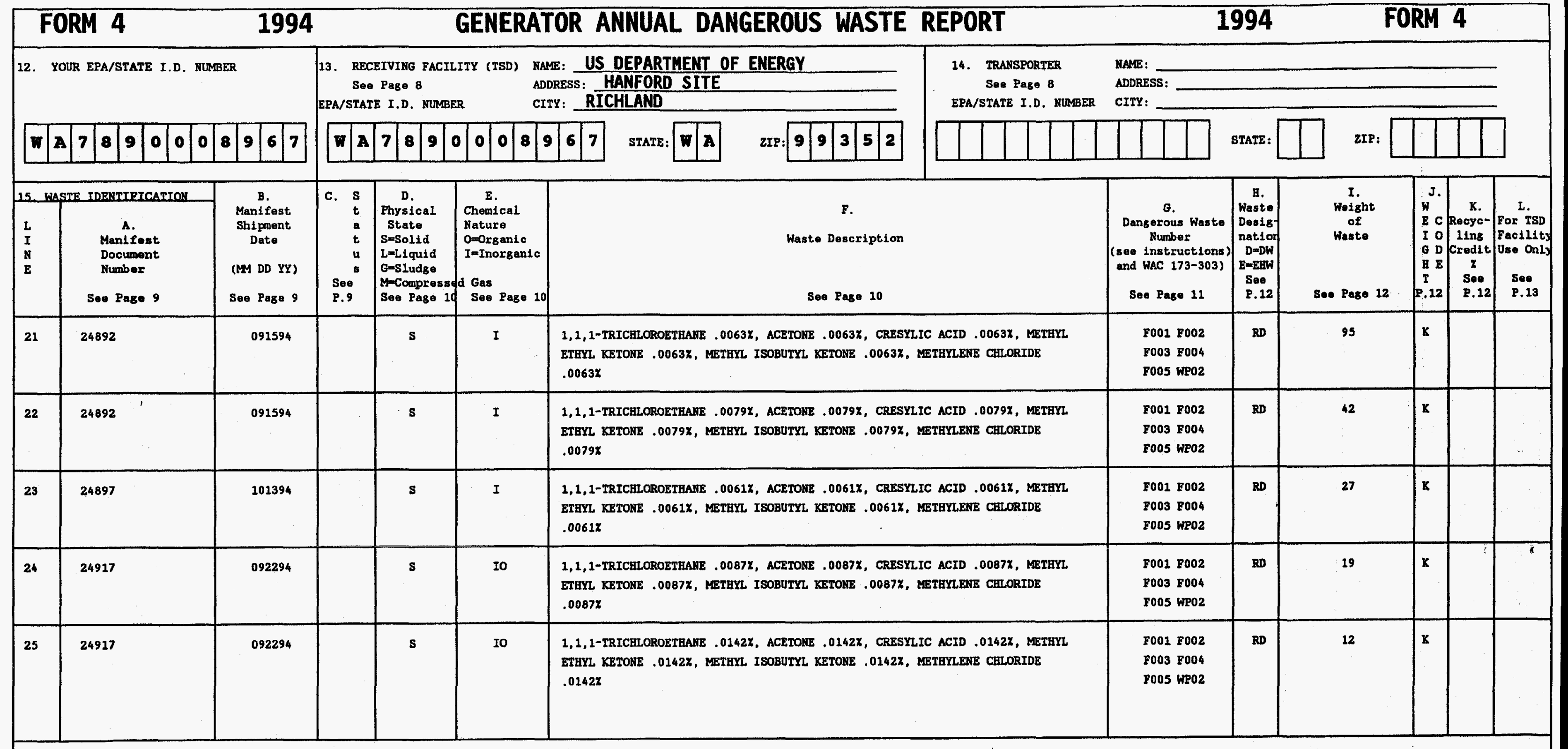

16. COMMENTS (Enter information by section and/or 11ne number--See Page 13). FACILITY: 241C

PAGE 71 OF 161 


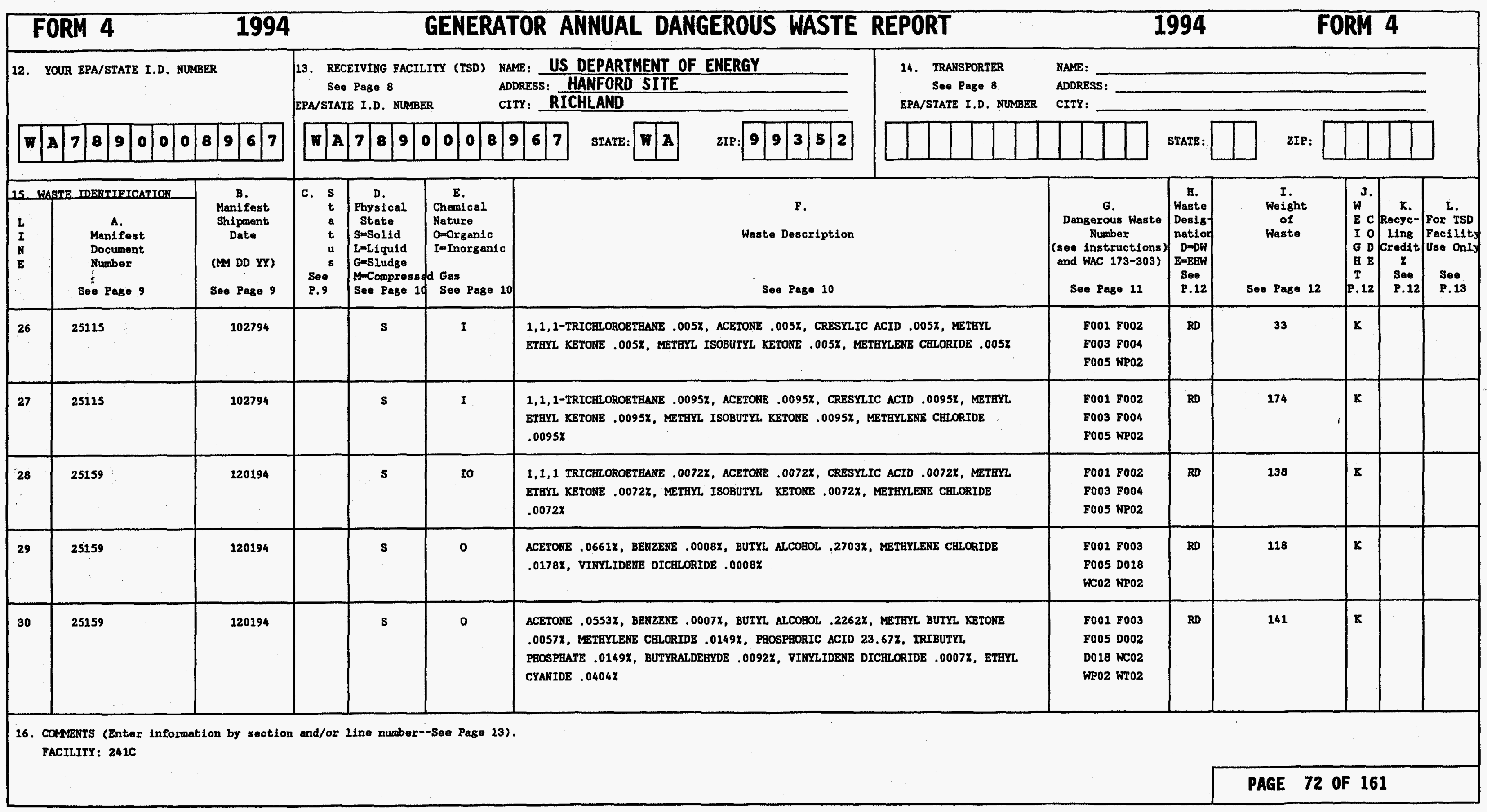




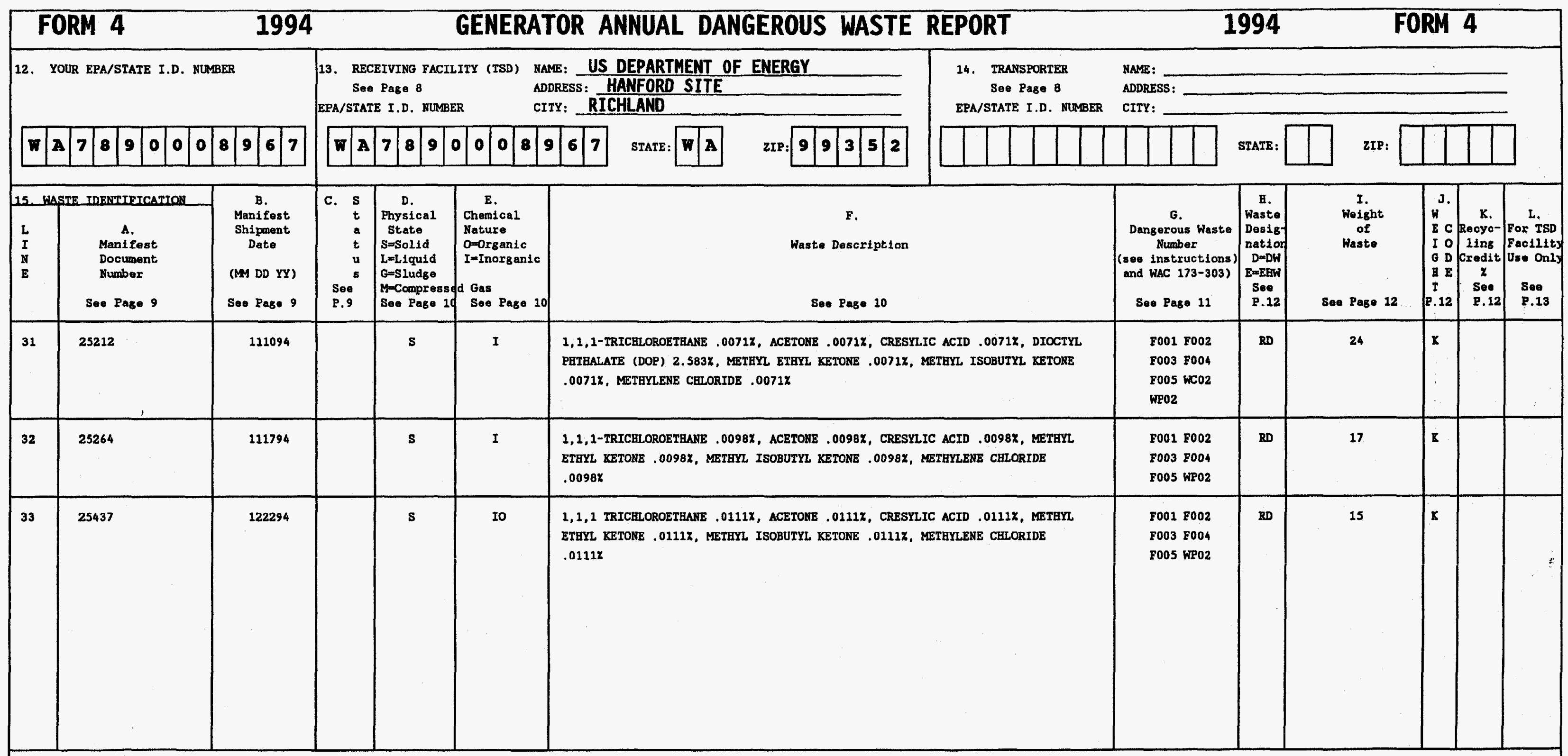

16. CoMvents (Enter information by section and/or line number--See Page 13). FACILITY: 241C 


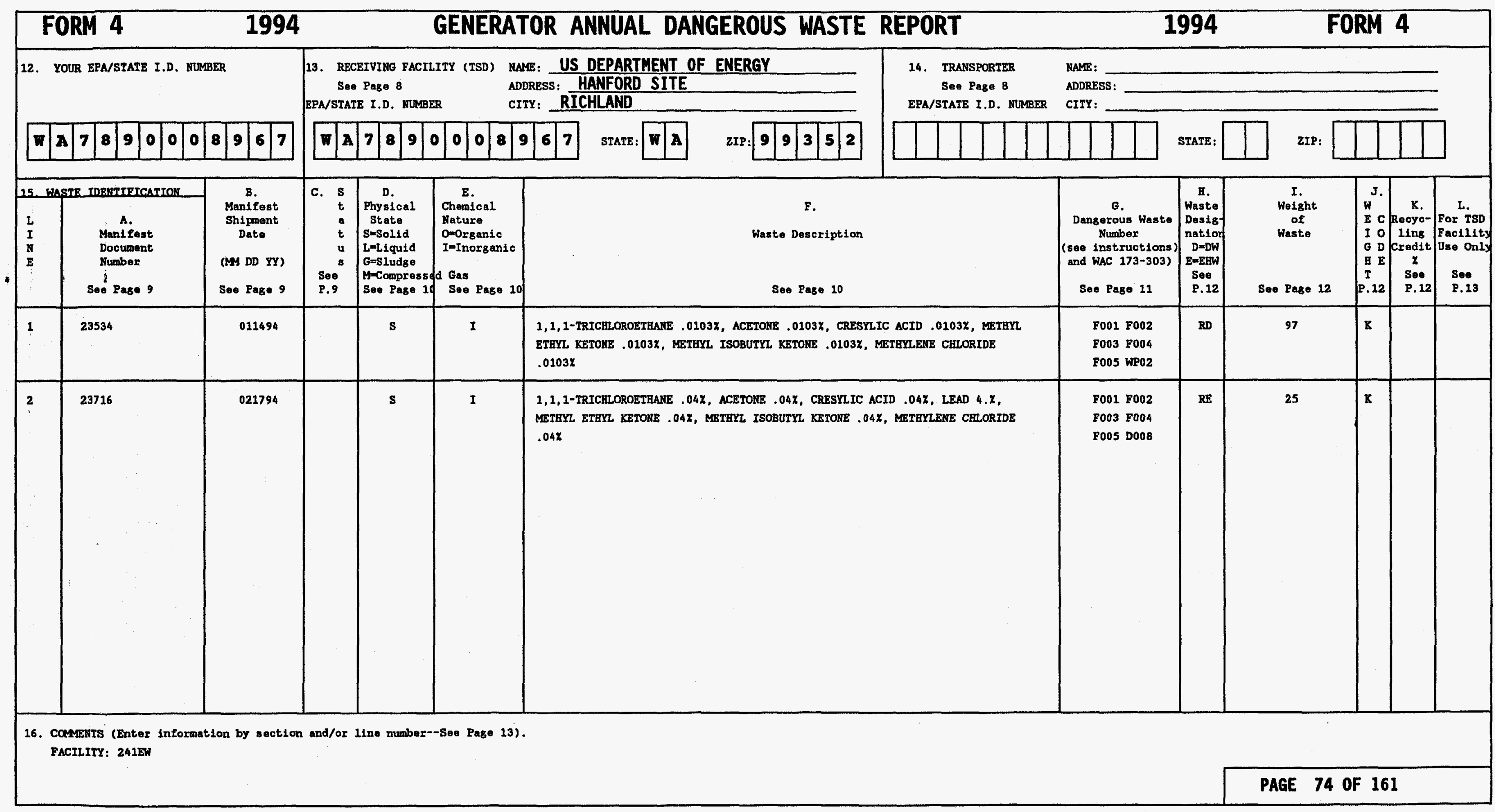




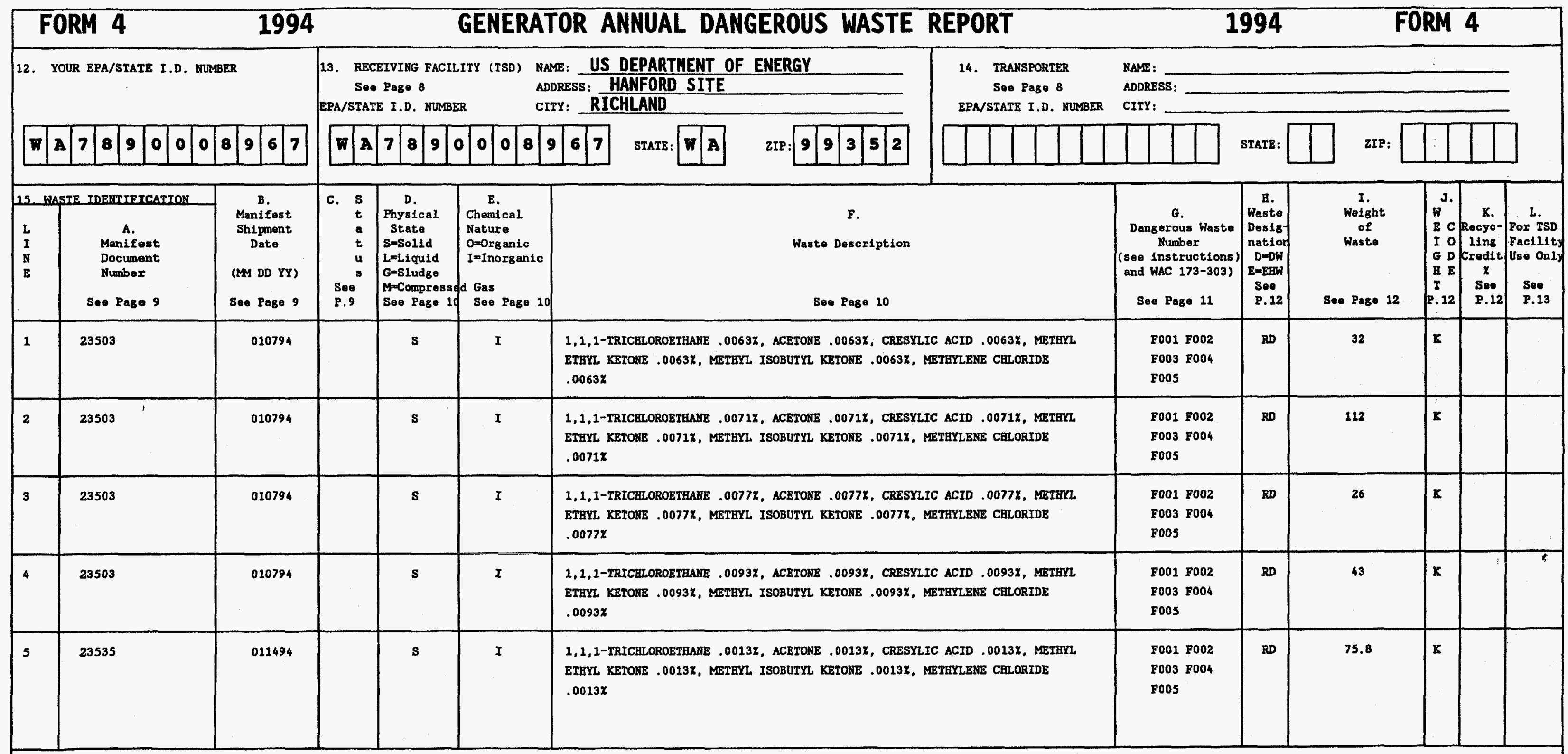

16. CONMENTS (Enter information by section and/or 11ne number--See Page 13), FACILITY: $241 S$ 


\begin{tabular}{|c|c|c|c|c|c|c|c|c|c|c|c|c|c|}
\hline & RM 4 & 1992 & & & GENERAT & OR ANNUAL DANGEROUS WASTE & REPORT & & $\overline{994}$ & & $\overline{R M}$ & & \\
\hline & JUR EPA/STATE I.D. & & $\begin{array}{l}\text { 13. } \\
\text { REC } \\
\text { See } \\
\text { EPA/STAT }\end{array}$ & $\begin{array}{l}\text { PIVING FACIL } \\
\text { Page } 8 \\
\text { EE I.D. NUMBE }\end{array}$ & $\begin{array}{ll}\text { CITY (TSD) } & \text { NA } \\
\mathbb{E R} & \text { AD } \\
\mathbb{E R}\end{array}$ & $\begin{array}{l}\text { E: US DEPARTMENT OF ENERGY } \\
\text { ORES: } \frac{\text { HANFORD SITE }}{\text { RICHLAND }}\end{array}$ & $\begin{array}{l}\text { 14. TRANSPORTER } \\
\text { See Page } 8 \\
\text { EPA/STATE I.D. NU }\end{array}$ & $\begin{array}{l}\text { NAME: } \\
\text { ADDRESS: } \\
\text { CITY: }\end{array}$ & & & & - & \\
\hline $\mathbf{n}$ & \begin{tabular}{l|l|l|l|l}
7 & 8 & 9 & 0 & 0 \\
\end{tabular} & \begin{tabular}{|l|l|l|l|}
8 & 9 & 6 & 7 \\
\end{tabular} & $\mathbf{w}$ & 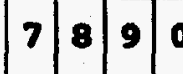 & \begin{tabular}{l|l|l|l|l}
0 & 0 & 0 & 8 & $:$
\end{tabular} & 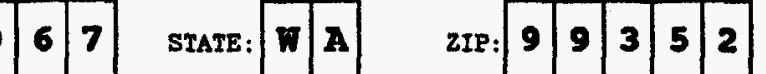 & & & staTE: & zIP: & & & \\
\hline 15. & ITE TDERTIETCATtOr & & c. $s$ & & & & & & \begin{tabular}{|c|} 
站 \\
Waste
\end{tabular} & I. & w. & $x$ & \\
\hline $\begin{array}{l}2 \\
I \\
\text { I } \\
E\end{array}$ & $\begin{array}{l}\text { A. } \\
\text { Manifest } \\
\text { Document } \\
\text { Numbar } \\
\vdots . \\
\text { Soo Page } 9\end{array}$ & $\begin{array}{l}\text { Shlpoent } \\
\text { Date } \\
\text { (MM DD YY) } \\
\text { Soe Page } 9\end{array}$ & $\begin{array}{c}a \\
t \\
u \\
\text { Sees } \\
\text { p.9 }\end{array}$ & 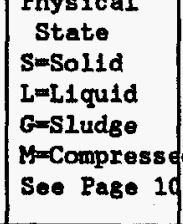 & $\begin{array}{l}\text { Nature } \\
\text { onorganic } \\
\text { ImInorganic } \\
\text { d Gas } \\
\text { See Page } 10\end{array}$ & $\begin{array}{l}\text { Waste Description } \\
\text { See Page } 10\end{array}$ & & $\begin{array}{c}\text { G. } \\
\text { Dangerous Waste } \\
\text { Number } \\
\text { (see Instructions) } \\
\text { and WAC 173-303) } \\
\text { Soe Page } 11\end{array}$ & 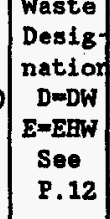 & $\begin{array}{l}\begin{array}{c}\text { Welght } \\
\text { of } \\
\text { Waste }\end{array} \\
\text { Soe Page } 12\end{array}$ & 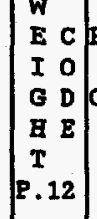 & $\begin{array}{c}\text { K. } \\
\text { Recye- } \\
\text { CIng } \\
\text { Credit } \\
z \\
\text { Se. } \\
\text { P.12 }\end{array}$ & \begin{tabular}{|l} 
For Tsp \\
Fac11145 \\
Uase Only \\
See \\
P.13
\end{tabular} \\
\hline 6 & 23535 & 011494 & & s & I & $\begin{array}{l}\text { 1,1,1-TRICHLOROETAANE .0025X, ACETONE .0025x, CRESY } \\
\text { ETHYL KETONE .0025X, METHYL ISOBUTYL KETONB .0025X, } \\
.0025 X\end{array}$ & $\begin{array}{l}\text { ACID .0025\%, METEYL } \\
\text { THYLNE CHLORIDE }\end{array}$ & $\begin{array}{l}\text { F001 F002 } \\
\text { F003 F004 } \\
\text { F005 }\end{array}$ & $\mathrm{RD}$ & 39.5 & $\mathrm{x}$ & & \\
\hline 7 & 23535 & 011494 & & s & I & $\begin{array}{l}\text { 1,1,1-TRICHLOROETAANE .0038X, ACETONE .0038X, CRESY } \\
\text { ETHYL KETONE .0038X, METGYL ISOBUTYL KRTONE .0038, } \\
.0038 Z\end{array}$ & $\begin{array}{l}\text { ACID .0038\%, METEYL } \\
\text { THYLENE CHLORIDE }\end{array}$ & $\begin{array}{l}\text { F001 F002 } \\
\text { F003 F004 } \\
\text { F005 }\end{array}$ & $\mathrm{RD}$ & 52.6 & $\mathrm{x}$ & & \\
\hline 8 & 23535 & 011494 & & $\mathbf{s}$ & I & 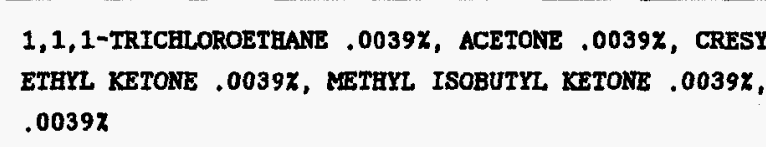 & $\begin{array}{l}\text { ACID . 0039X, METEYL } \\
\text { TAYLENE CBLORIDE }\end{array}$ & $\begin{array}{l}\text { F001 F002 } \\
\text { F003 F004 } \\
\text { F005 }\end{array}$ & RD & 25.4 & $\mathrm{k}$ & & \\
\hline 9 & 23535 & 011494 & & $\mathbf{s}$ & I & $\begin{array}{l}\text { 1,1,1-TRICHLOROETAANE .0042X, ACETONE .0042X, CRESY } \\
\text { ETHYL KETONE .0042X, METRYL ISOBUTYL KETONE, .0042X, } \\
.0042 X\end{array}$ & $\begin{array}{l}\text { ACID . DOA2X, METHYL } \\
\text { ARLENE CRLORIDE }\end{array}$ & $\begin{array}{l}\text { F001 F002 } \\
\text { F003 F004 } \\
\text { F005 }\end{array}$ & RD & 23.6 & $\mathrm{x}$ & & \\
\hline 10 & 23535 & 011494 & & s & I & 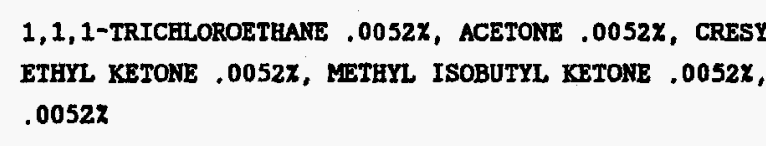 & $\begin{array}{l}\text { ACTD .0052x, METEM } \\
\text { GMENE CHEORIDE }\end{array}$ & $\begin{array}{l}\text { F001 F002 } \\
\text { F003 F004 } \\
\text { F005 }\end{array}$ & $\mathrm{RD}$ & 19.1 & $\mathrm{k}$ & & \\
\hline & $\begin{array}{l}\text { NTS (Enter } 1 \\
\text { ITY: } 2415\end{array}$ & se se & & & Page & & & & & & & & \\
\hline & & & & & & & & & & PAGE 76 & 161 & & \\
\hline
\end{tabular}




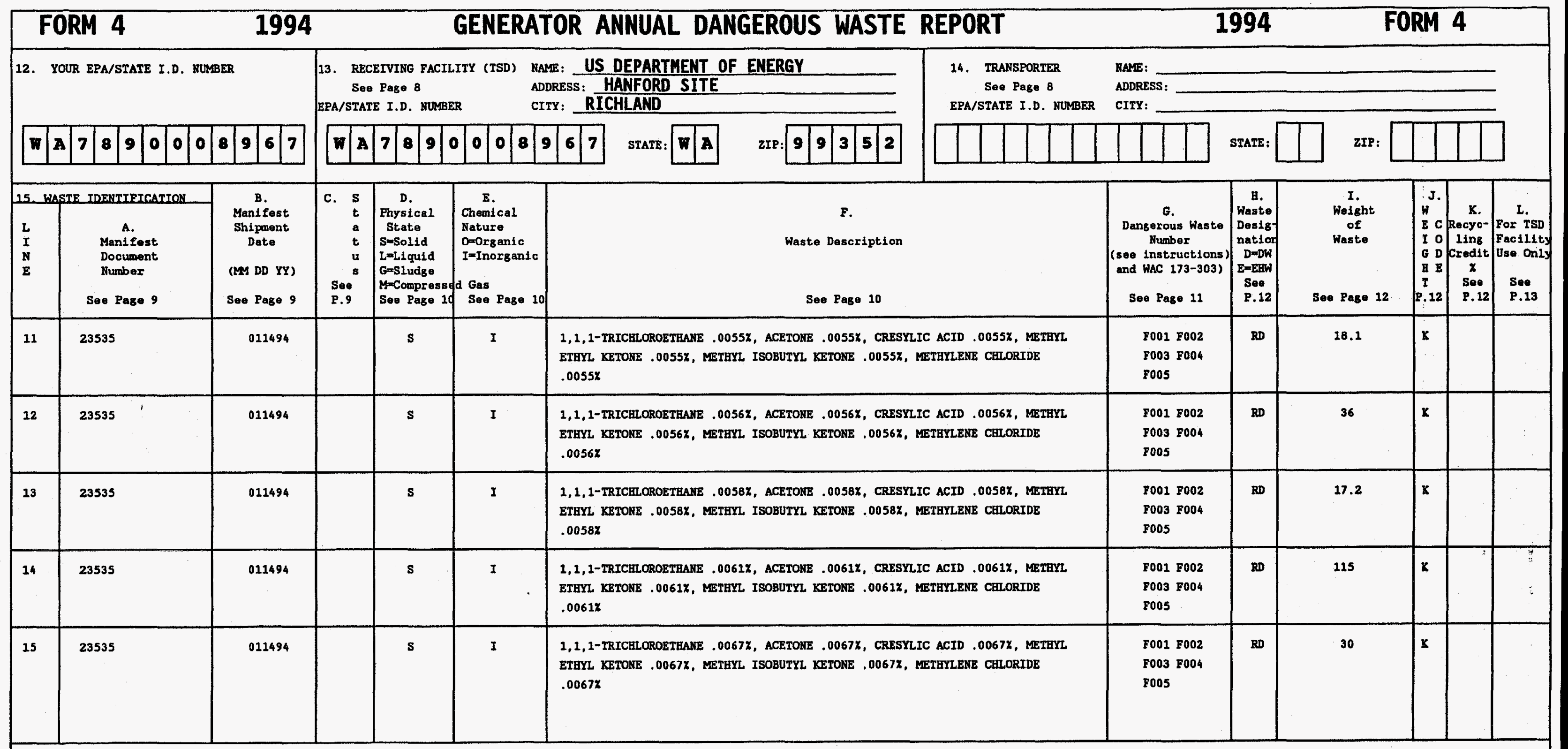

16. COMMENTS (Enter information by section and/or 11ne number--See Page 13). FACILITY: 2415 


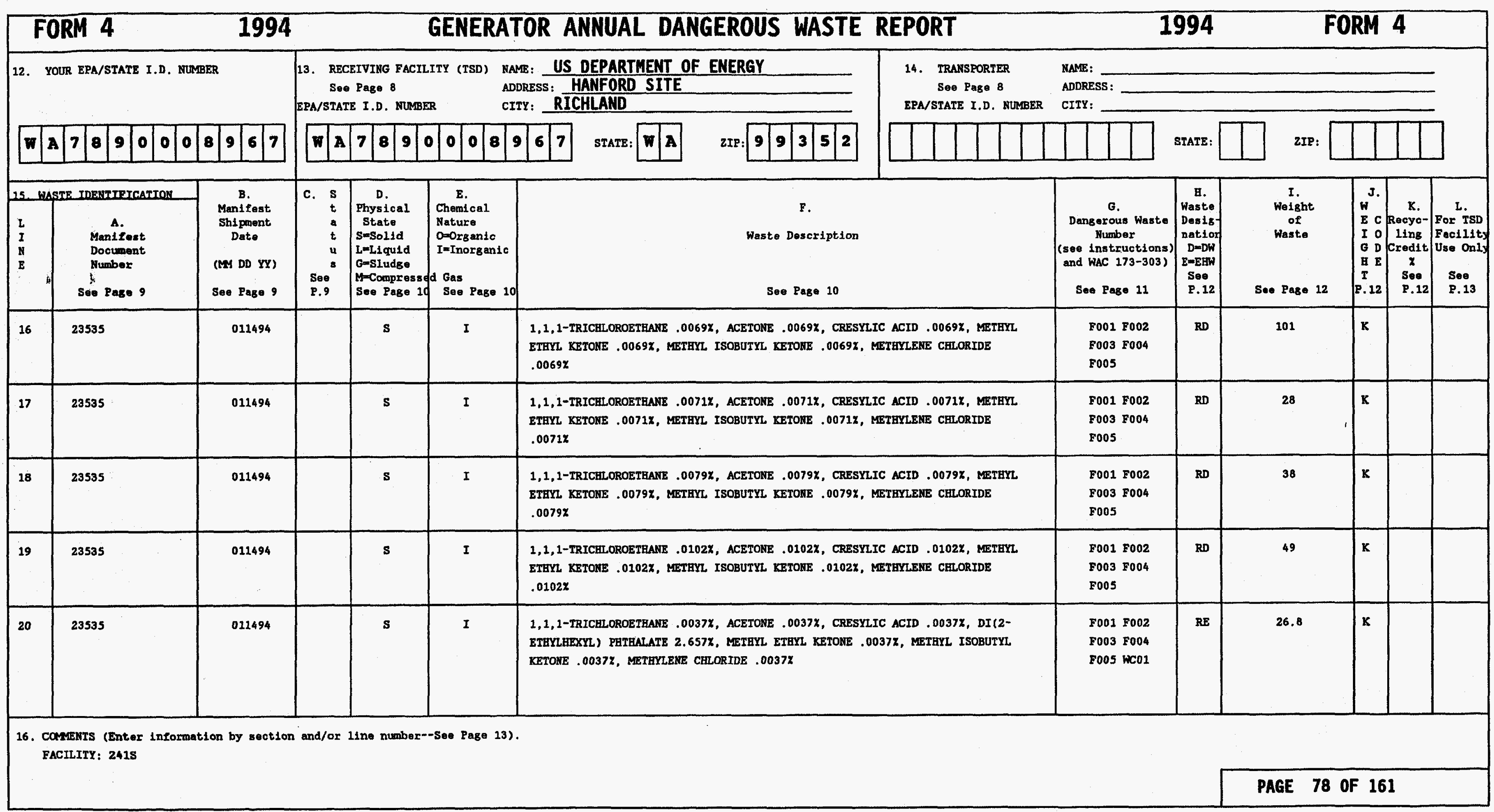




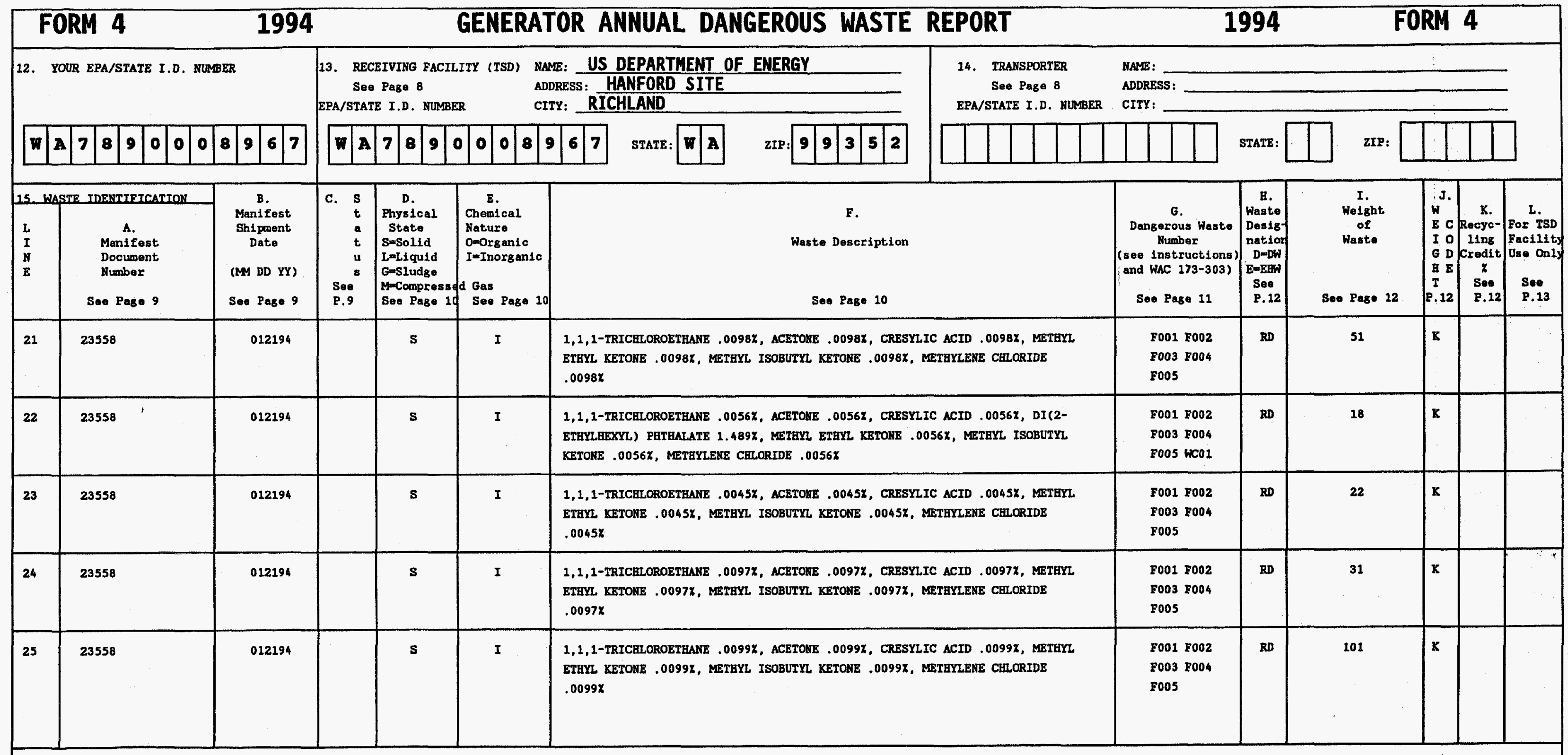

16. COMMENTS (Enter information by section and/or 11ne number--See Page 13). FACILITY: $241 \mathrm{~S}$

PAGE 79 OF 161 


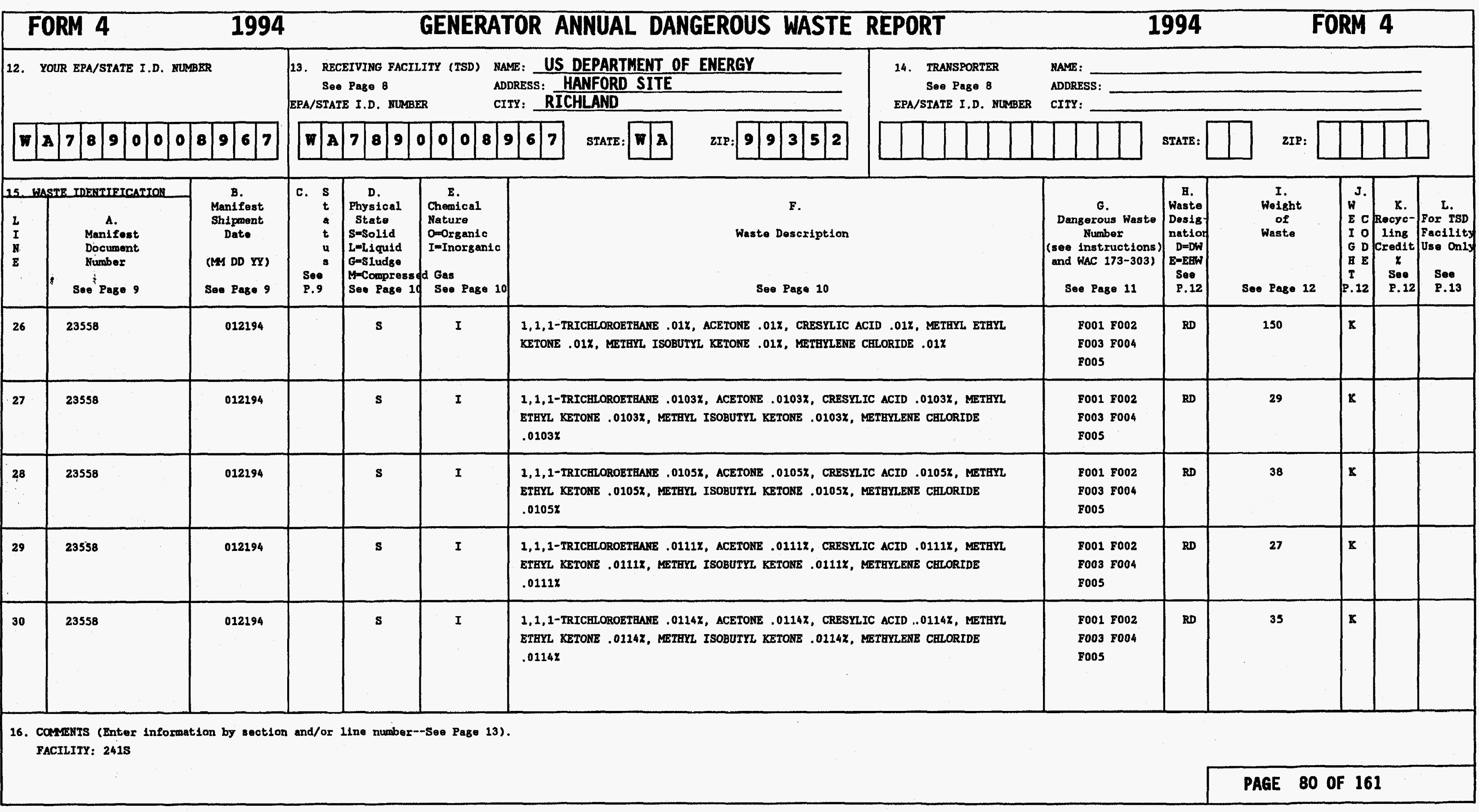




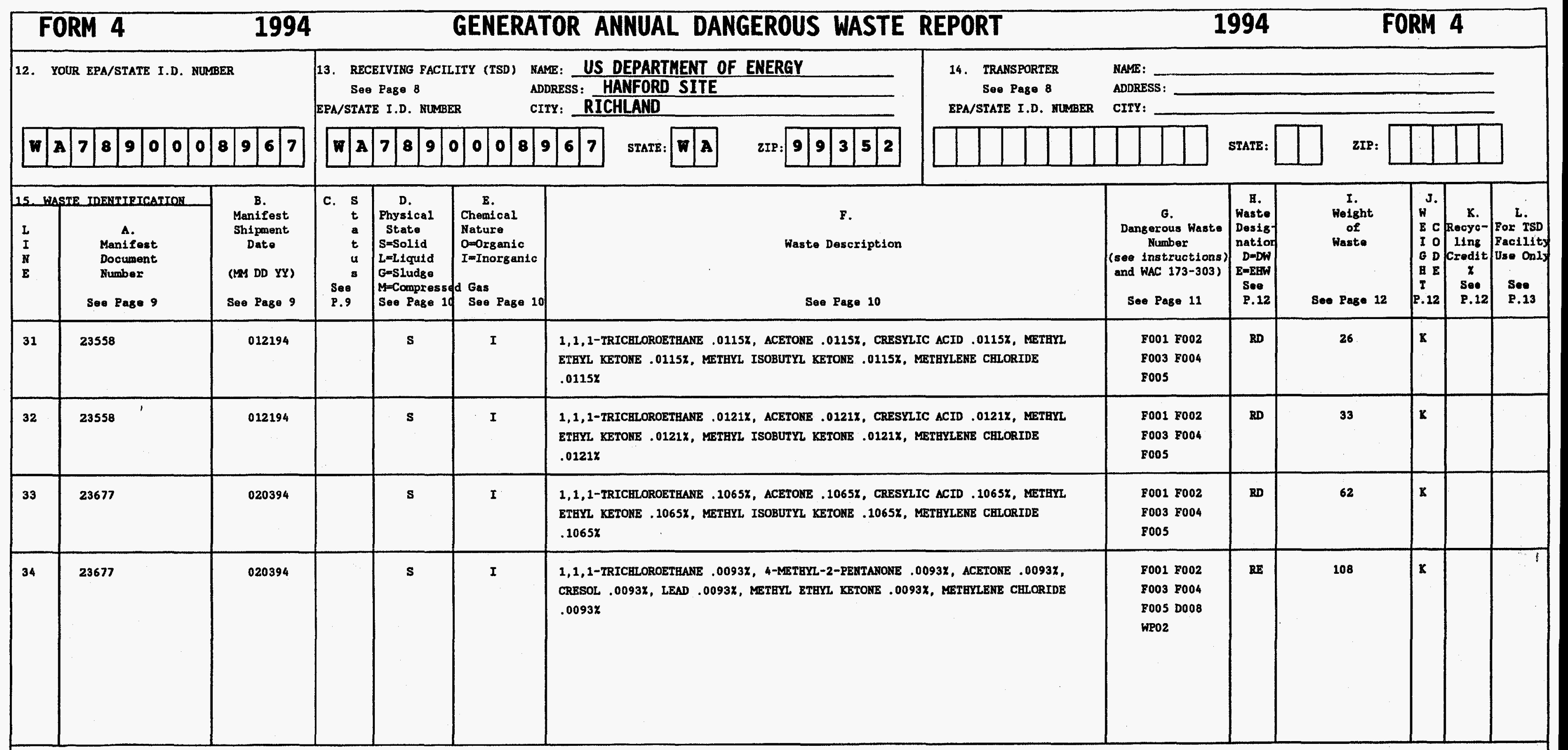

16. Comerrs (Enter information by section and/or line number--See Page 13). FACILITY: 241S

PAGE 81 OF 161 


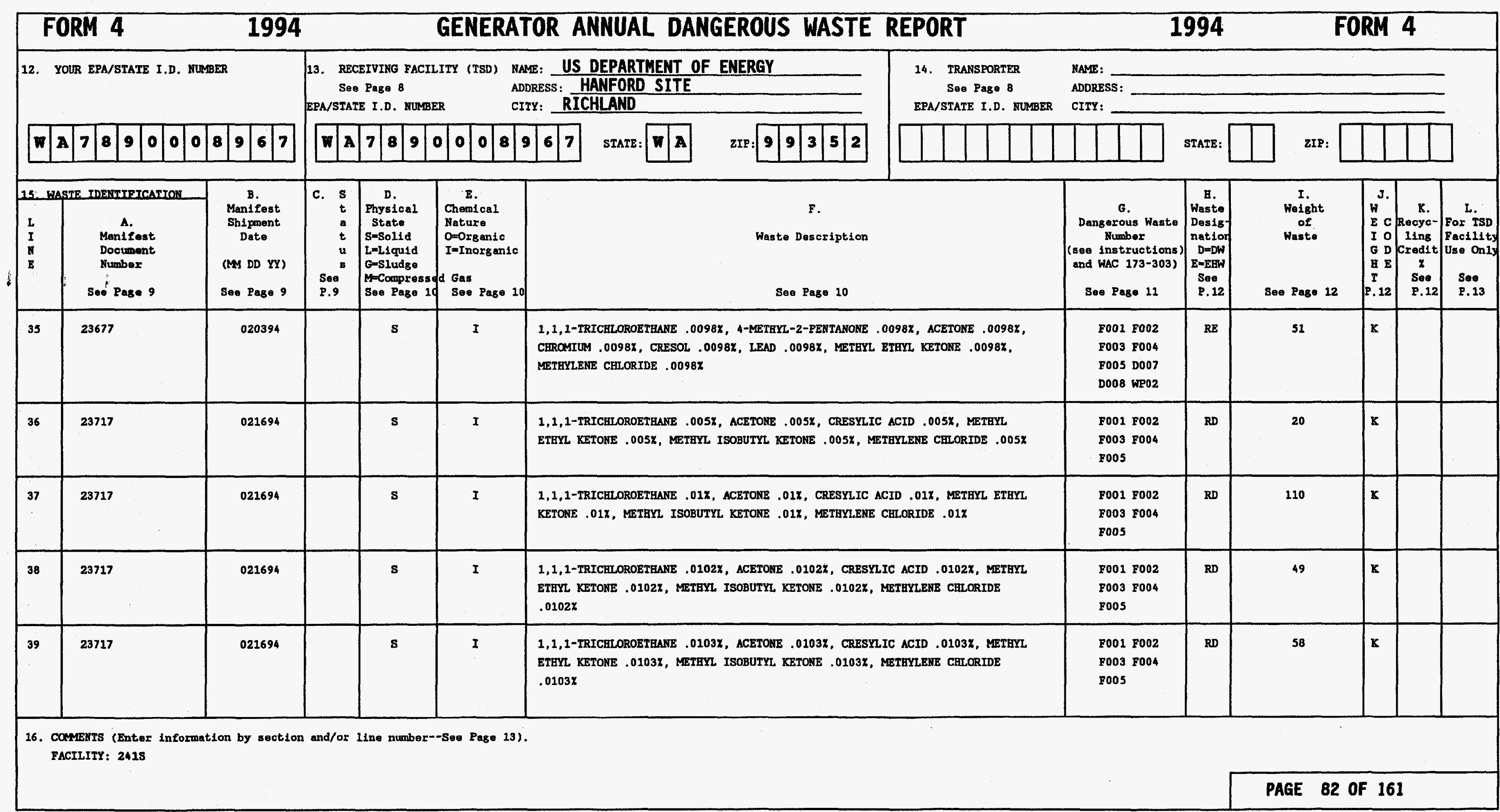




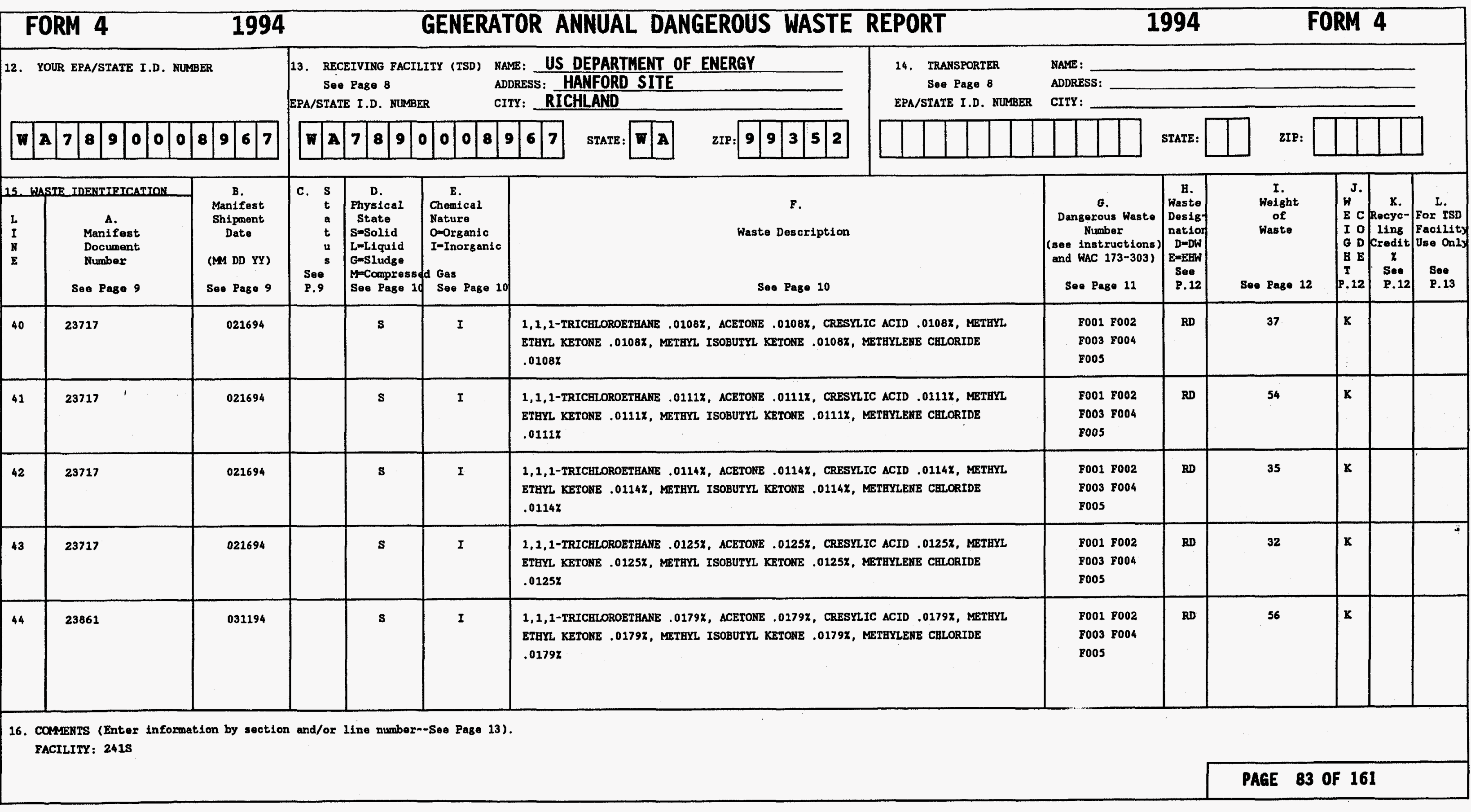




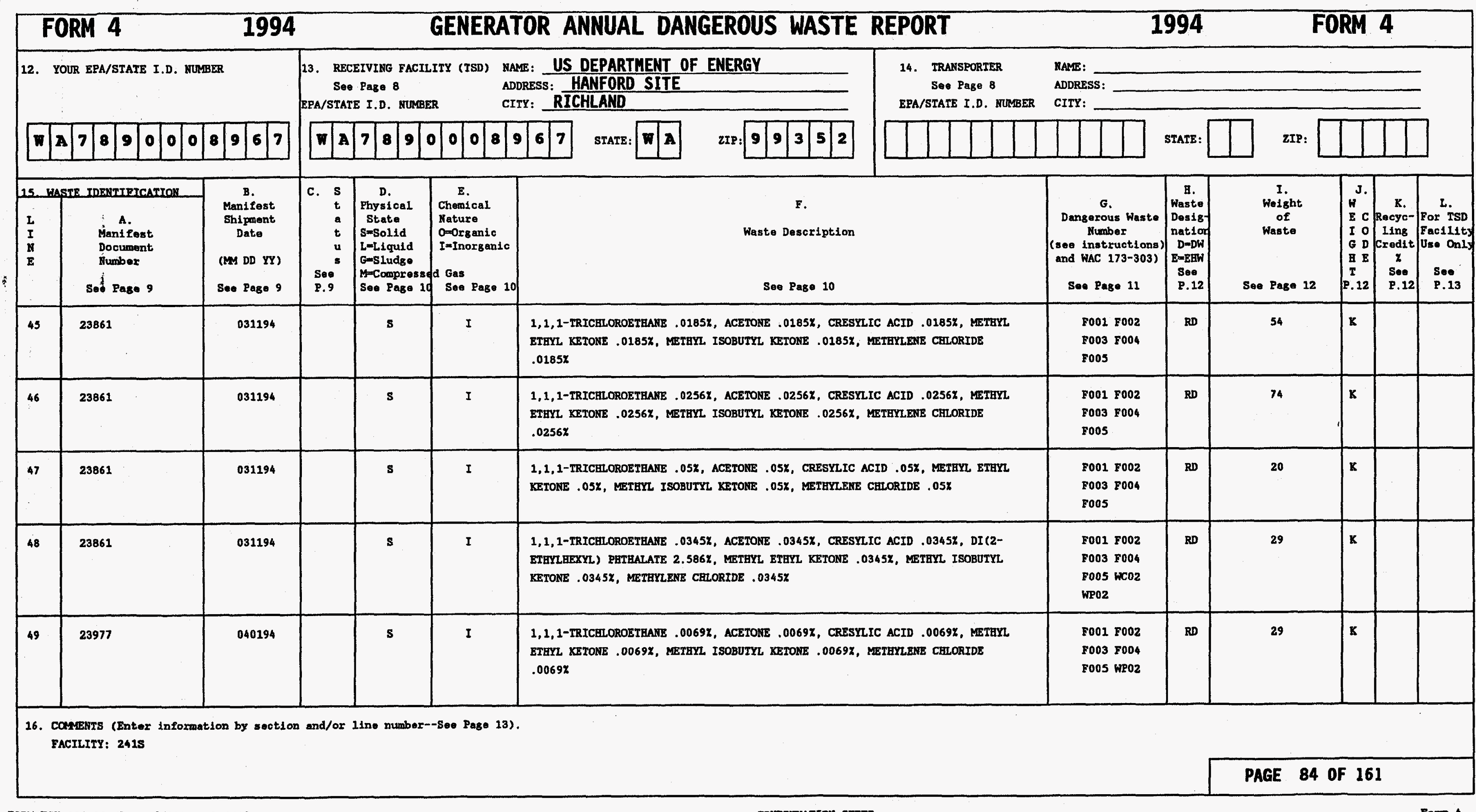




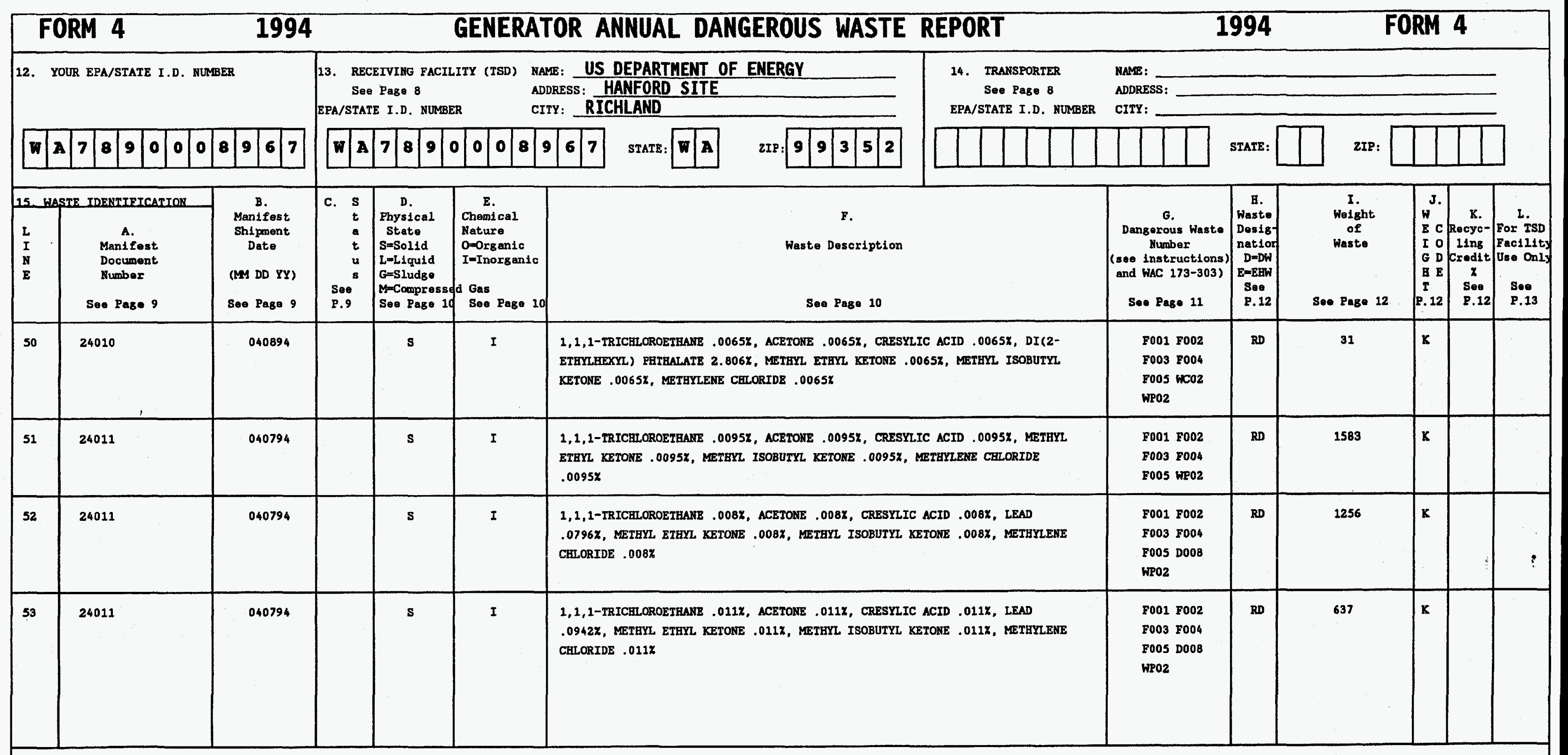

16. COMENTS (Enter information by seotion and/or line number--See Page 13). FACILITY: 2413

PAGE 85 OF 161 


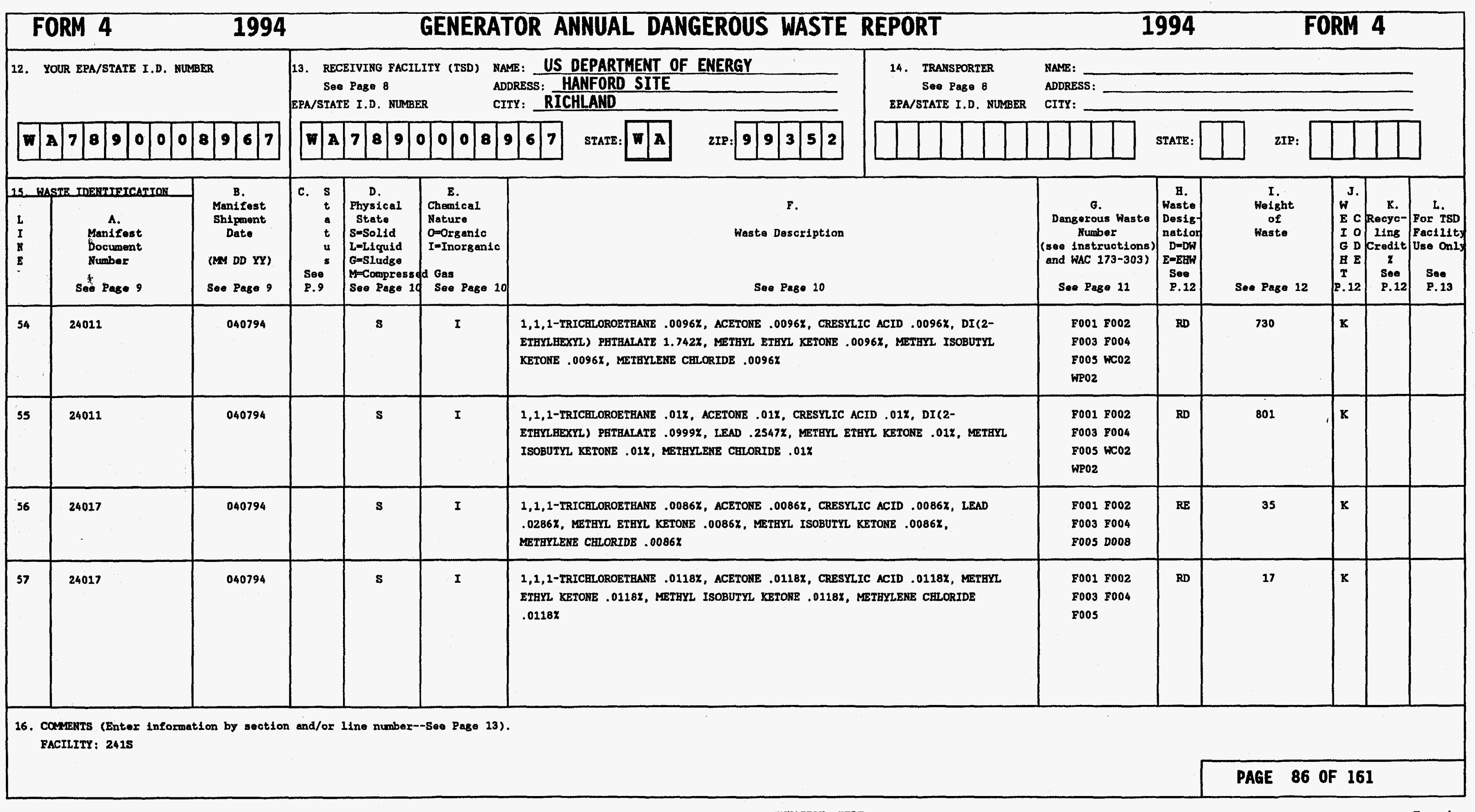




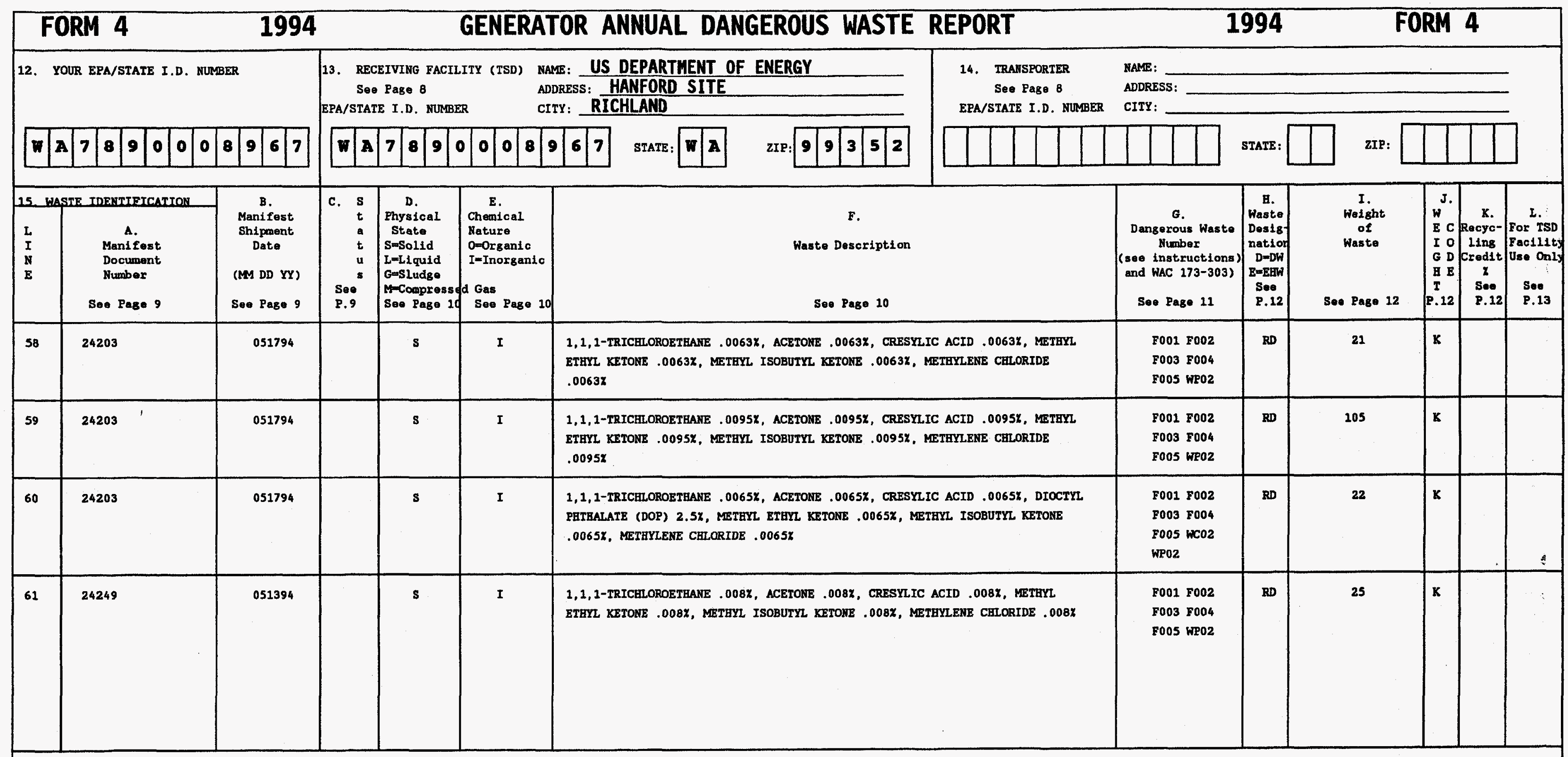

16. CoMmenTS (Enter information by section and/or line number--See Page 13). FACILITY: $241 \mathrm{~S}$ 


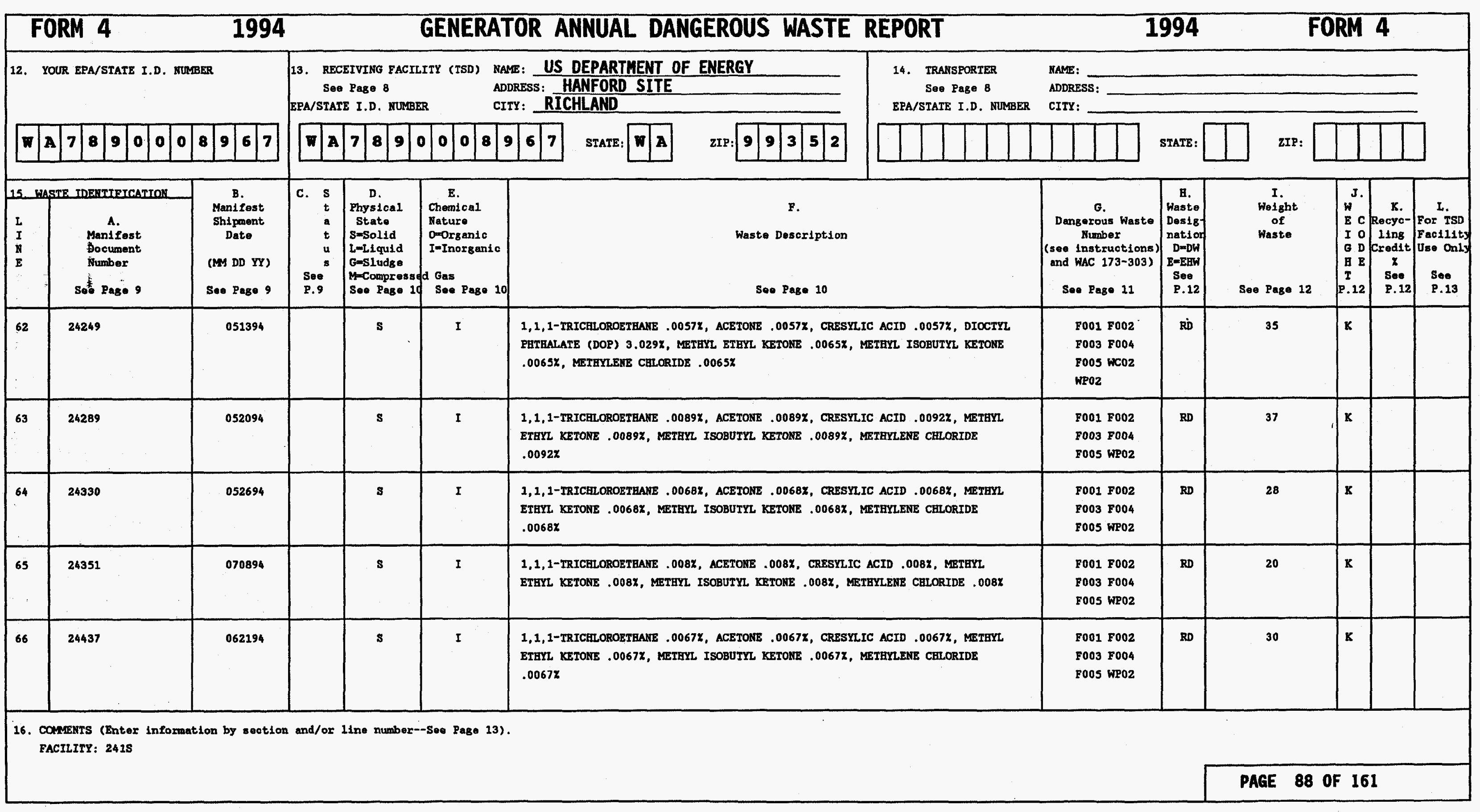




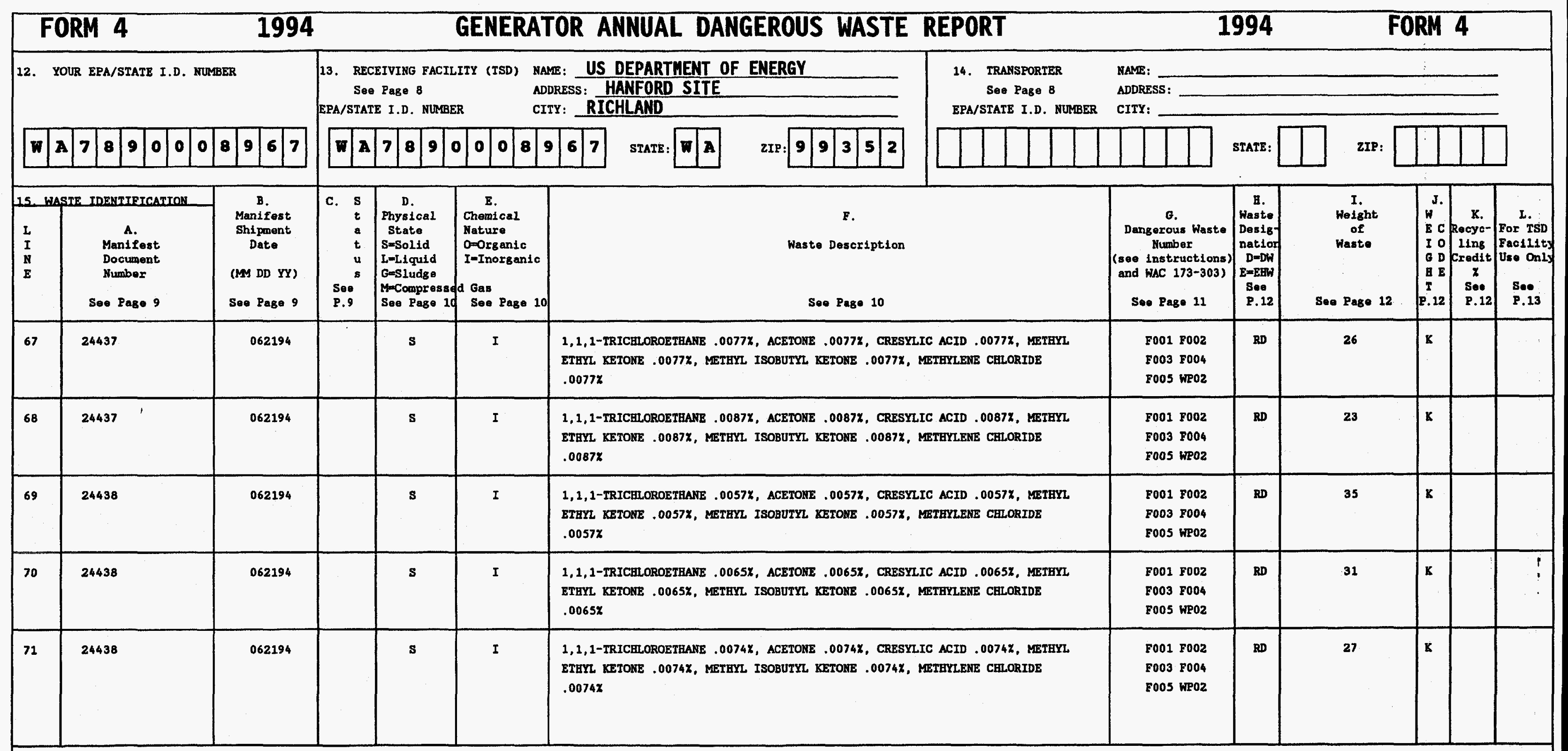

16. conerewrs (Enter information by section and/or 1ine number--See Page 13). FACILITY: $241 \mathrm{~s}$ 


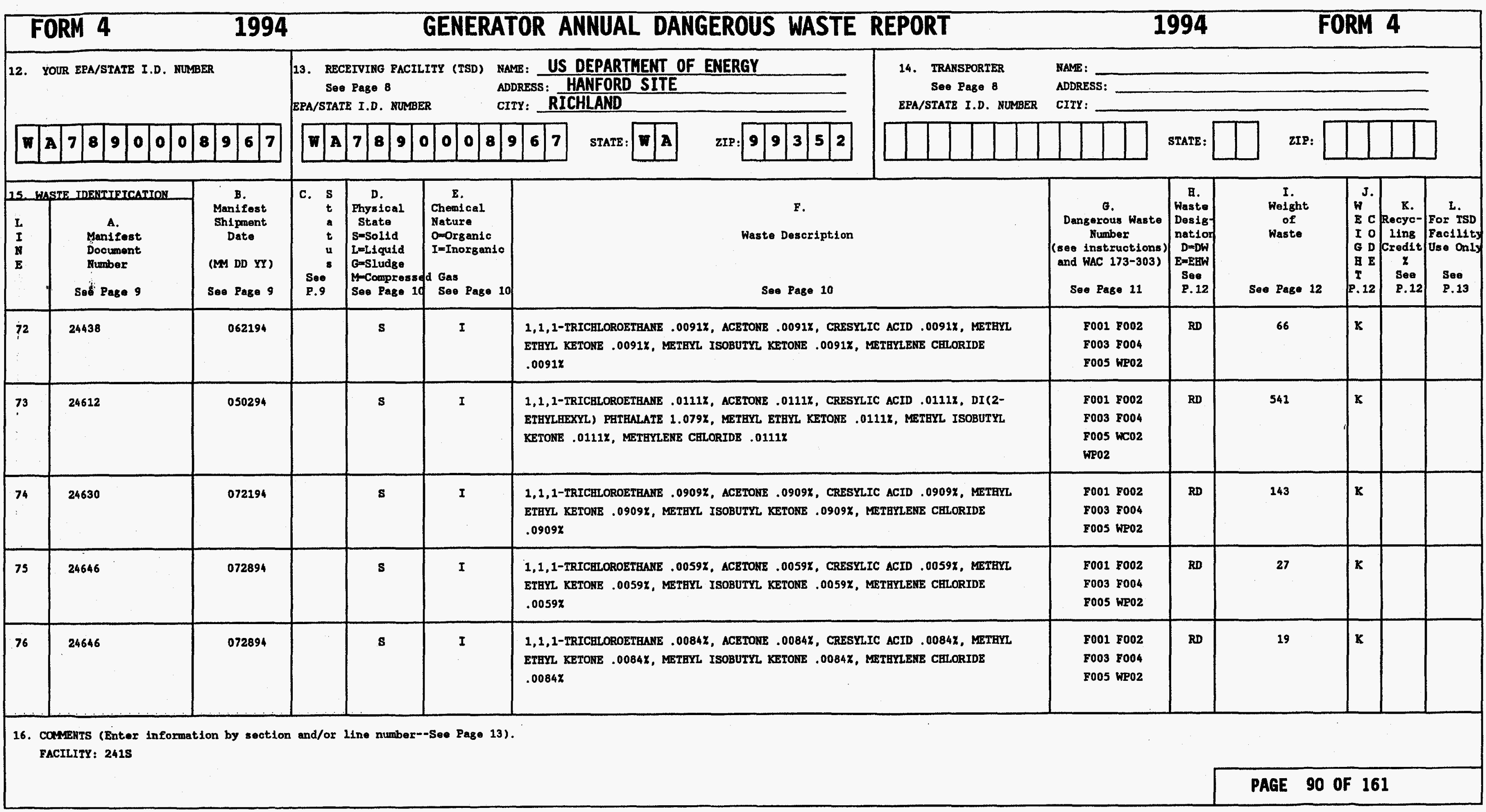




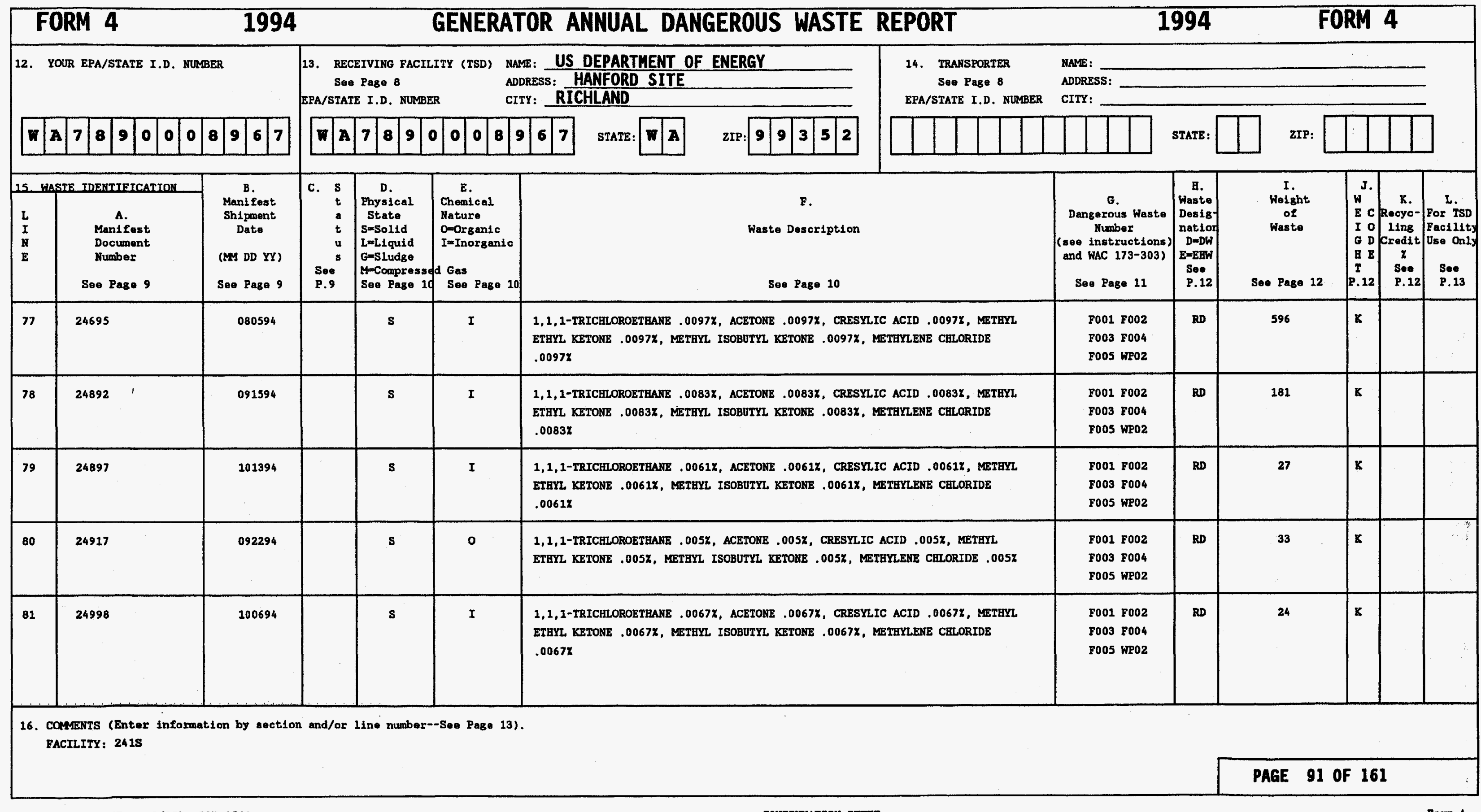




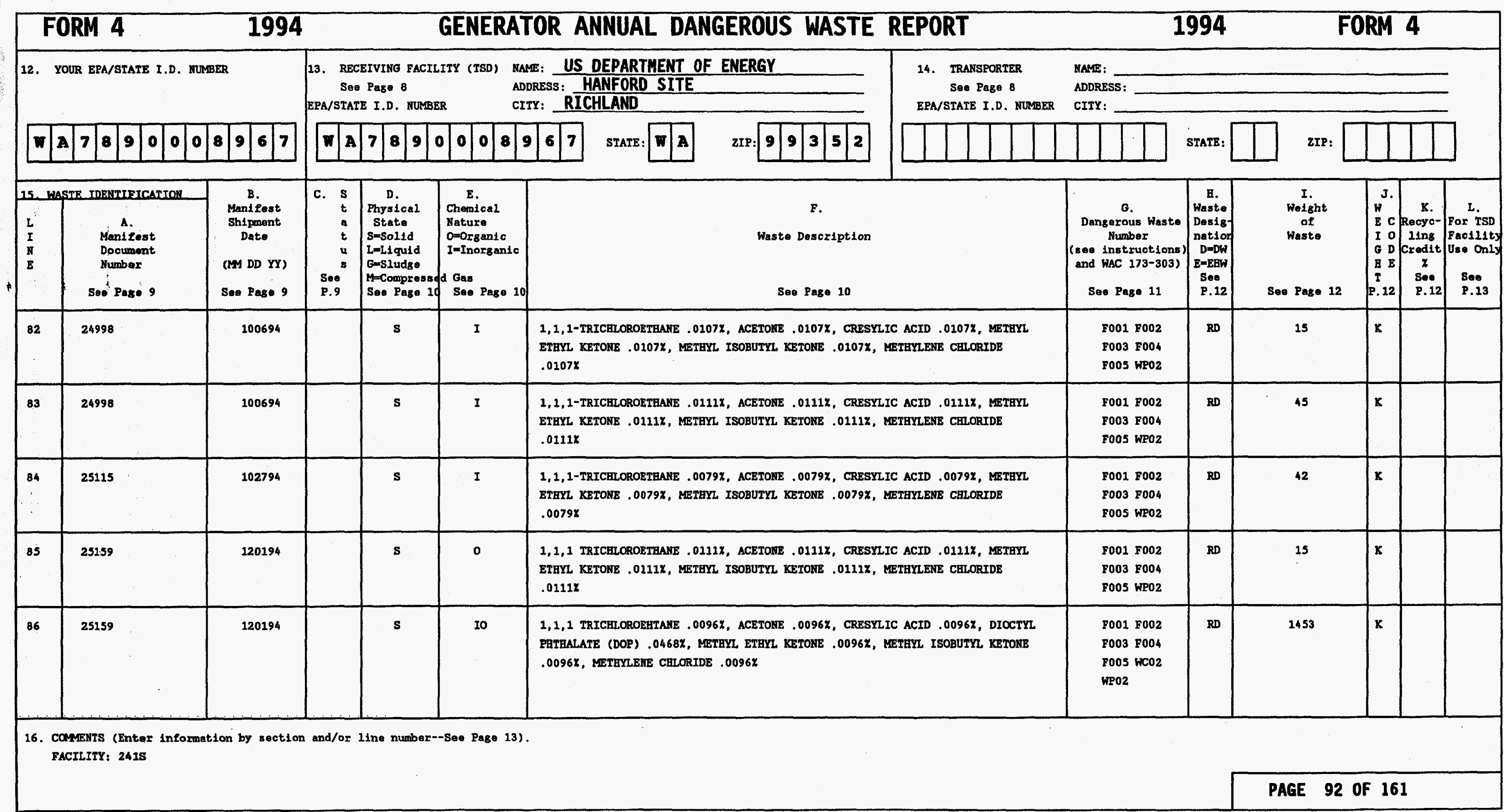




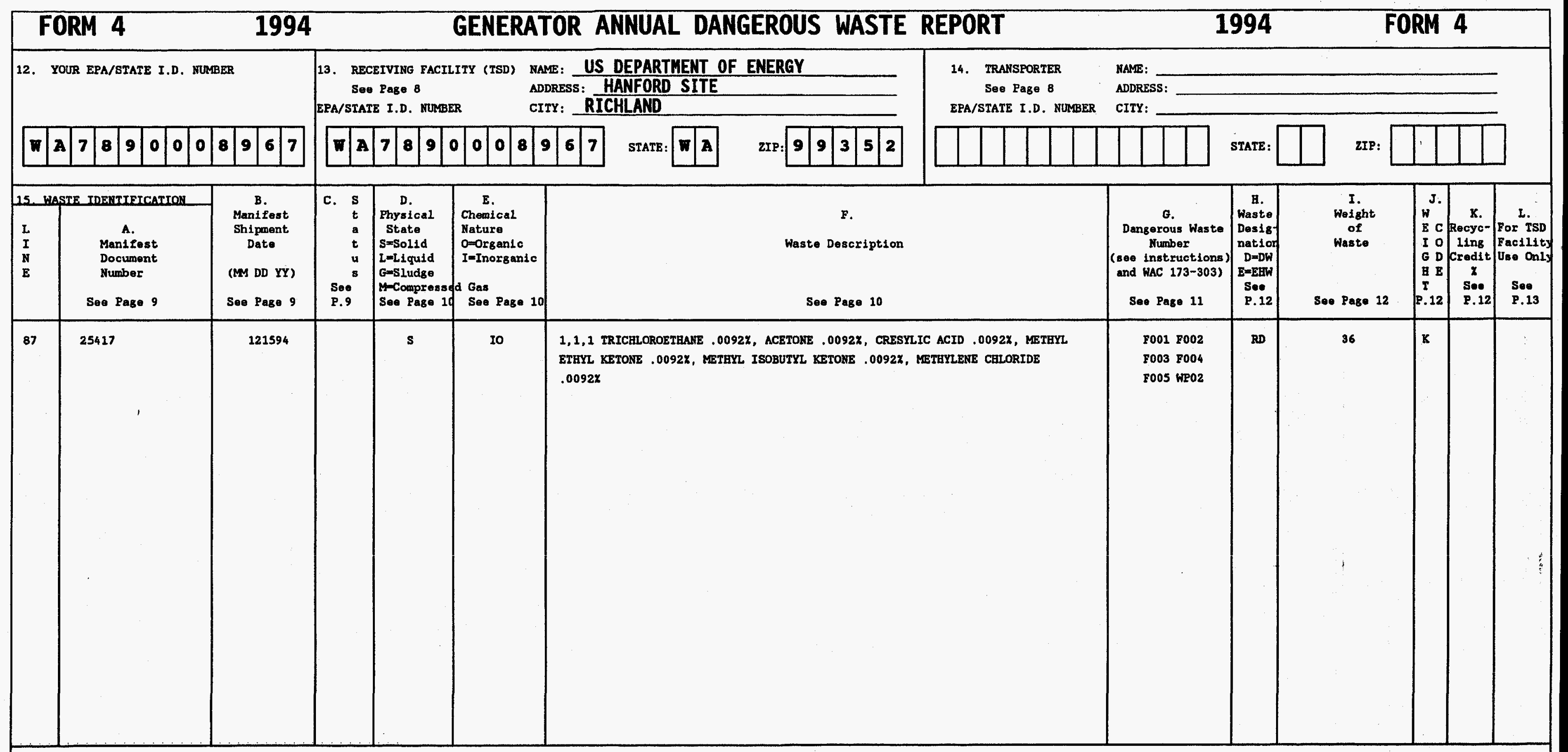

16. COMENTS (Enter information by section and/or 11ne number--See Page 13) FACILITY: 241S 


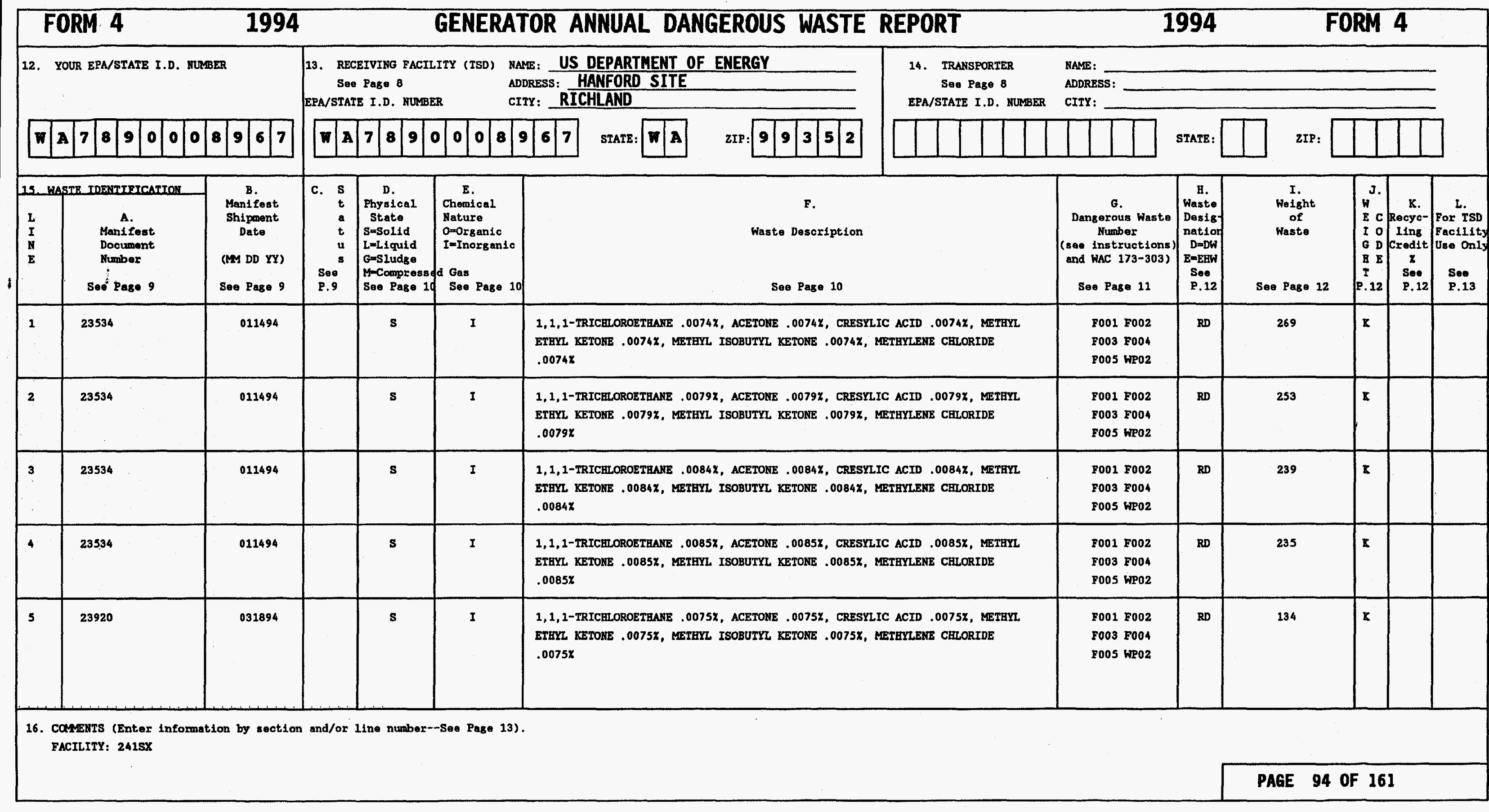




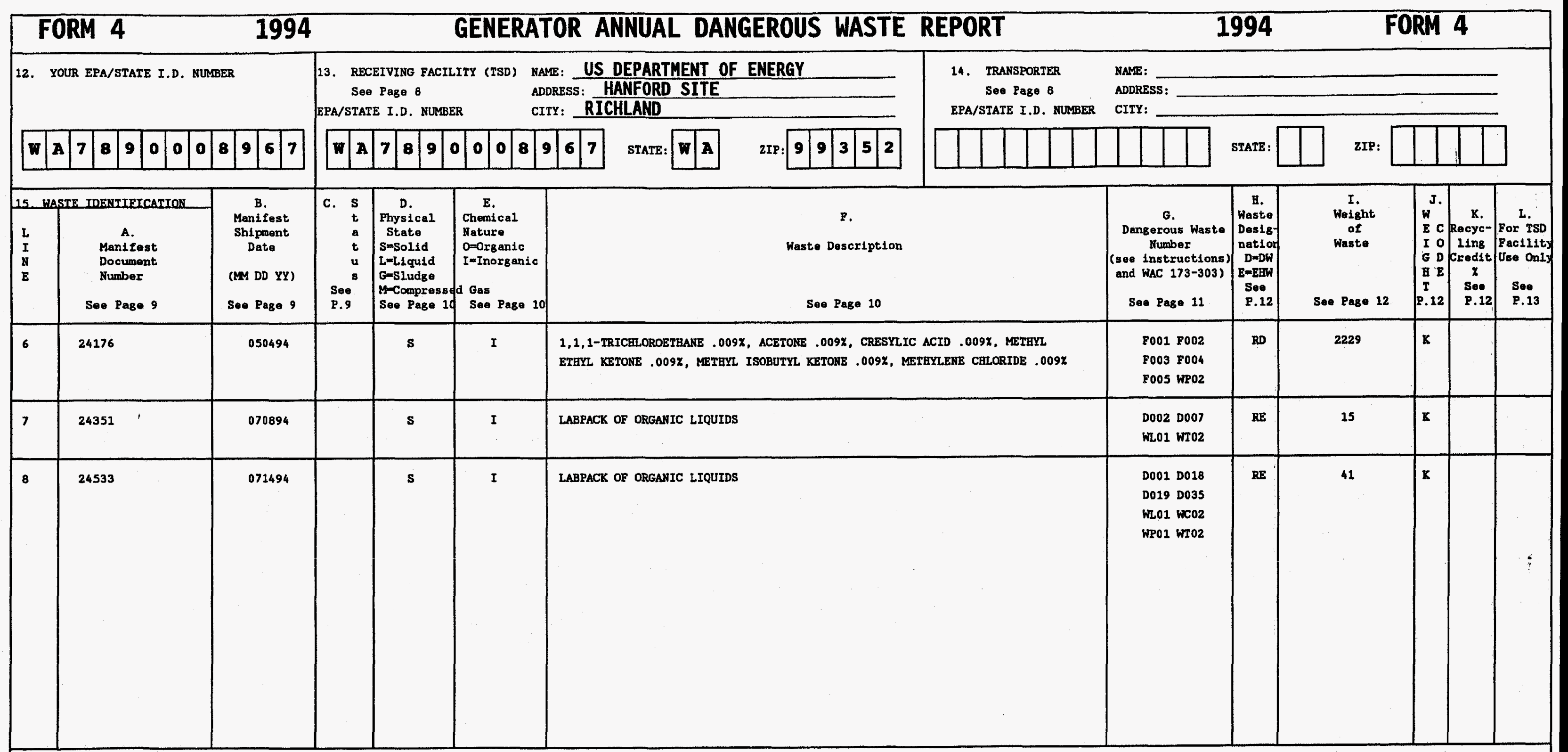

16. CanENTS (Enter information by section and/or 1ine number--Soe Paga 13). FACILITY: 241SX

PAGE 95 OF 161 


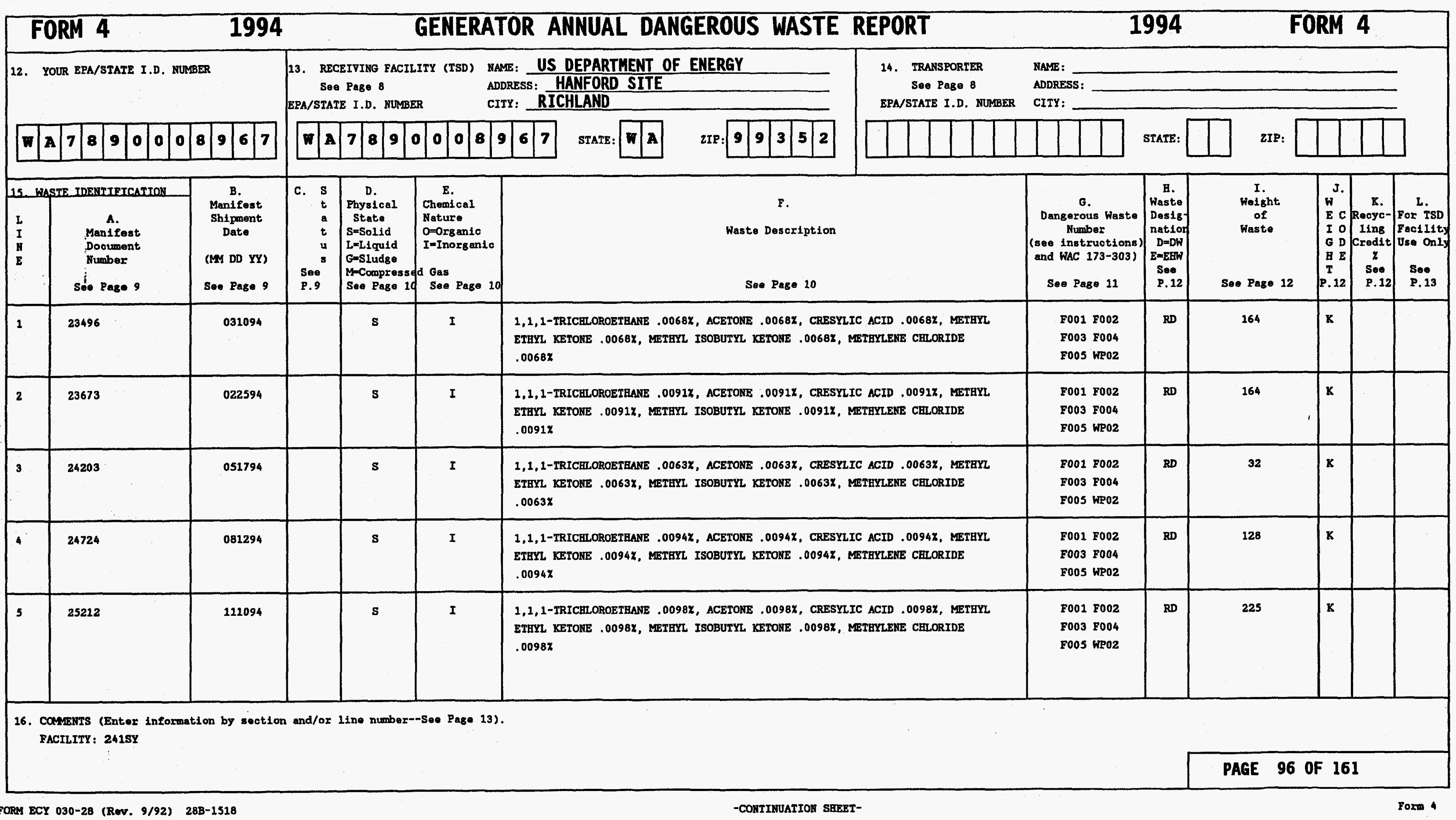




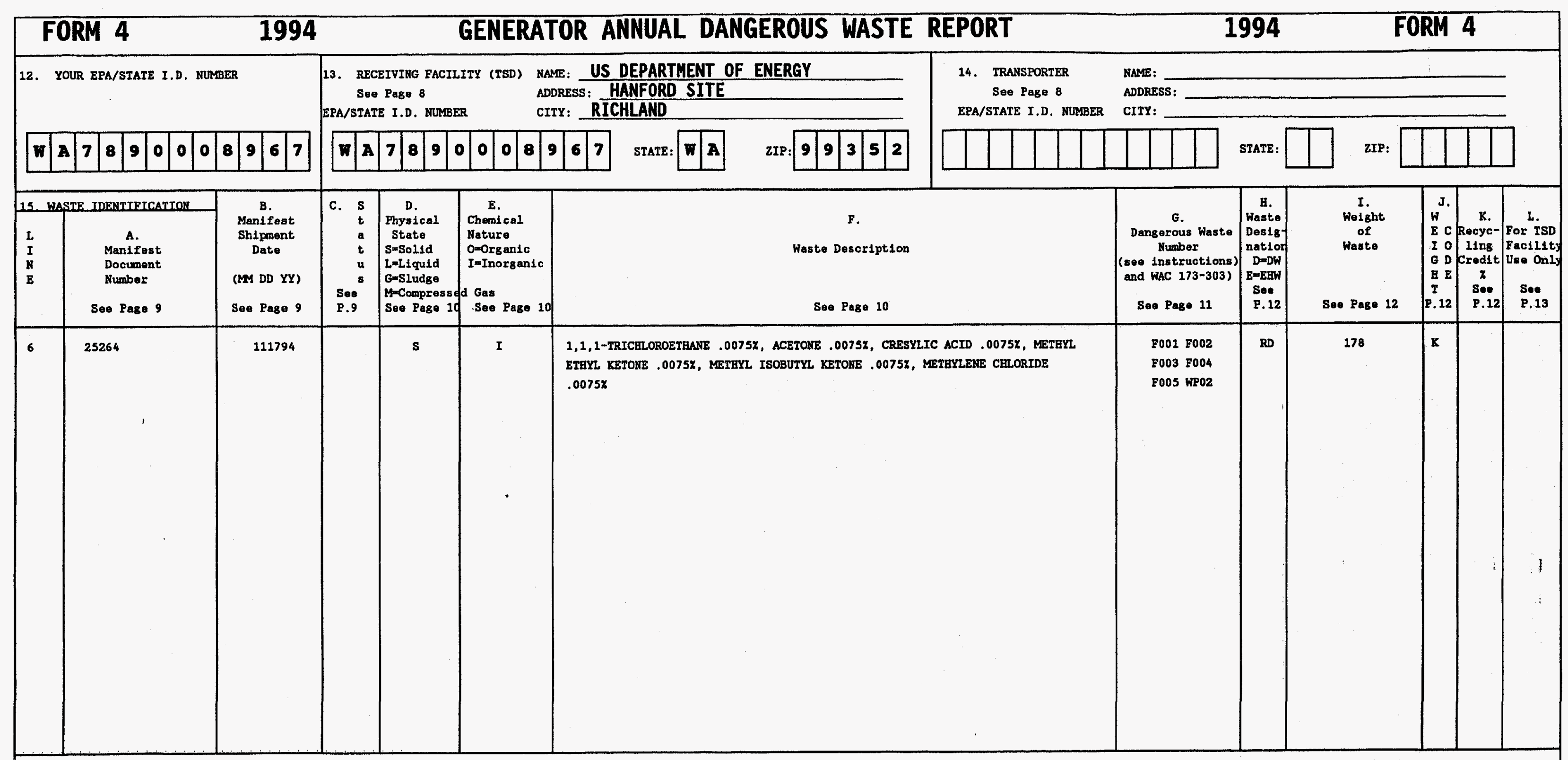

16. connexrs (Enter Information by section and/or 11ne number--Sea Page 13)

FACIIITY: 241SY

PAGE 97 OF 161 


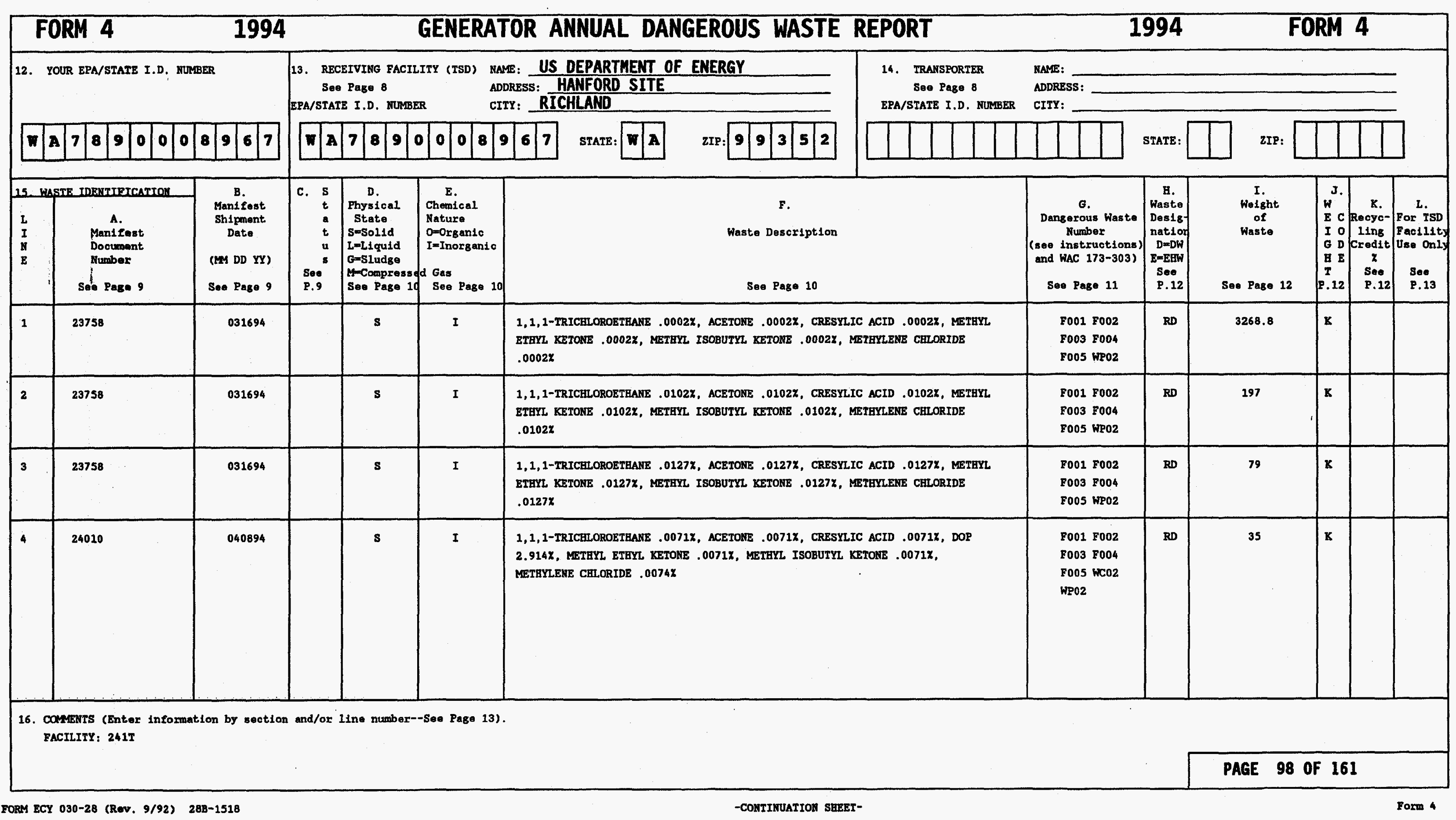




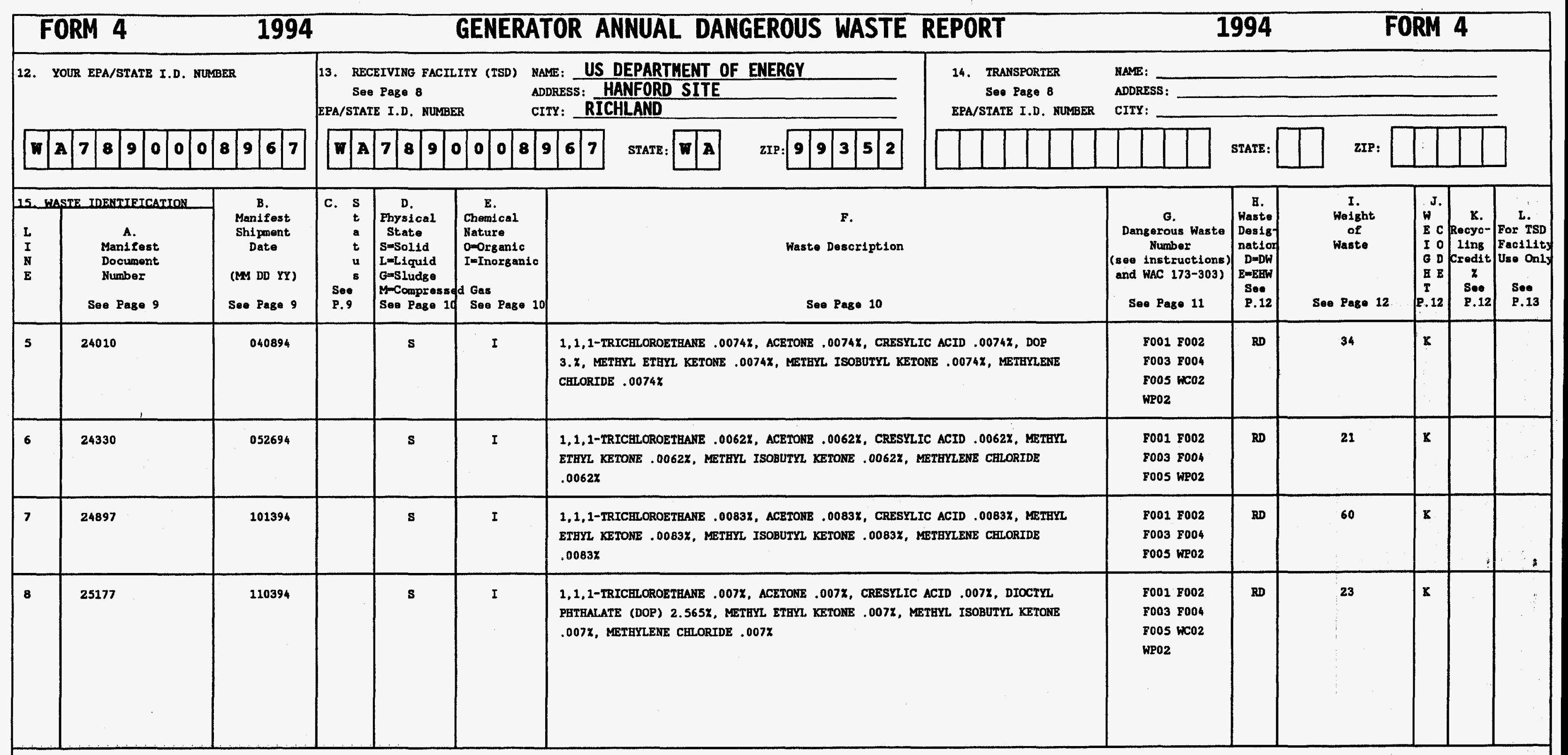

16. COMnENTS (Enter information by section and/or line number--Sea Page 13). FACILITY: $241 \mathrm{~T}$ 


\begin{tabular}{|c|c|c|c|c|c|c|c|c|c|c|c|c|c|c|c|c|c|}
\hline \multicolumn{4}{|c|}{ FORM 4} & \multicolumn{3}{|r|}{1994} & \multicolumn{5}{|c|}{ GENERATOR ANNUAL DANGEROUS WASTE REPORT } & & 994 & \multicolumn{4}{|c|}{ FORM 4} \\
\hline \multicolumn{7}{|c|}{ 12. YOUR EPA/STATE I.D. NUMBER } & \multicolumn{4}{|c|}{$\begin{array}{ll}\text { 13. RECEIVING FACILITY (TSD) } & \text { NAME: } \\
\text { Seo Page B } & \text { ADDRESS: HANFORTMENT OF ENERGY } \\
\text { EPA/STATE I.D. NUMBER } & \text { CITY: RICHLAND } \\
\end{array}$} & $\begin{array}{l}\text { 14. TRANSPORTER } \\
\text { See Page } 8 \\
\text { EPA/STATE I.D. NUMBER }\end{array}$ & \multicolumn{5}{|l|}{$\begin{array}{l}\text { NAME: } \\
\text { ADDRESS : } \\
\text { CITY: }\end{array}$} & \\
\hline$n$ & \begin{tabular}{l|l}
$\mathbf{A}$ & 7
\end{tabular} & 8 & 90 & 0 & $0 \mid 8$ & \begin{tabular}{|l|l|l|}
8 & 6 & 7 \\
\end{tabular} & $\mathbf{m} \mathbf{A}$ & \begin{tabular}{|l|l|l|l|l|l|l|l|l|l|l}
7 & 8 & 9 & 0
\end{tabular} & \begin{tabular}{l|l|l|l|l|}
0 & 0 & 0 & 8 & 9
\end{tabular} & 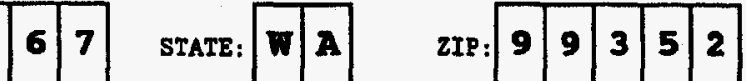 & & & STATE : & ZIP: & & & \\
\hline \multicolumn{6}{|c|}{ 15. GLASTE IDENTIETCATION } & \multirow[b]{2}{*}{$\begin{array}{c}\text { B. } \\
\text { Manifest } \\
\text { Shipment } \\
\text { Date } \\
\text { (MM DD YY) } \\
\text { Soe Page } 9\end{array}$} & \multirow[b]{2}{*}{ 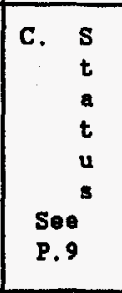 } & \multirow[b]{2}{*}{\begin{tabular}{|c|} 
D. \\
Physical \\
State \\
S=Solid \\
L-Liquid \\
G=Sludge \\
M-Compressed \\
Soo Page 10 \\
\end{tabular}} & \multirow[b]{2}{*}{\begin{tabular}{|l|}
\multicolumn{1}{|c|}{ E. } \\
Chemical \\
Nature \\
O=0rganic \\
I=Inorganic \\
d Gas \\
Soo Page 10 \\
\end{tabular}} & \multirow{2}{*}{\multicolumn{2}{|c|}{$\begin{array}{c}\text { F. } \\
\text { Waste Description }\end{array}$}} & \multirow[b]{2}{*}{$\begin{array}{c}\text { G. } \\
\text { Dangerous Waste } \\
\text { Number } \\
\text { (see Instructions) } \\
\text { and WAC 173-303) } \\
\text { Soe Page 11 } \\
\end{array}$} & \multirow[b]{2}{*}{\begin{tabular}{|c|} 
B. \\
Wasto \\
Desis \\
nation \\
D=DW \\
E=EEW \\
See \\
P.12 \\
\end{tabular}} & \multirow[b]{2}{*}{$\begin{array}{c}\begin{array}{c}\text { I. } \\
\text { Welght } \\
\text { of } \\
\text { Waste }\end{array} \\
\text { Soo Page } 12 \\
\end{array}$} & \multirow[b]{2}{*}{$\begin{array}{l}\mathrm{J} \\
\text { W } \\
\mathrm{E} \\
\mathrm{I} \\
\mathrm{C} \\
\mathrm{G} \\
\mathrm{B} \\
\mathrm{B} \\
\mathrm{T} \\
\mathrm{T} \\
\mathrm{P} .12\end{array}$} & \multirow[b]{2}{*}{\begin{tabular}{|c|} 
\\
K. \\
Rocyc- \\
ling \\
Credit \\
$x$ \\
See \\
$P .12$ \\
\end{tabular}} & \multirow[b]{2}{*}{\begin{tabular}{|l} 
L. \\
For TSD \\
Fac1lits \\
Use Onls \\
See \\
P.13
\end{tabular}} \\
\hline $\begin{array}{l}\text { I } \\
\text { I } \\
\text { E }\end{array}$ & $s$ & $\begin{array}{c}\text { Mo } \\
\text { Do } \\
\text { Nu } \\
\text { See }\end{array}$ & $\begin{array}{l}\text { A. } \\
\text { ififest } \\
\text { cument } \\
\text { mber } \\
\text { Page } 9\end{array}$ & & & & & & & & & & & & & & \\
\hline 1 & & 3534 & & & & 0.11494 & & $\mathbf{s}$ & I & $\begin{array}{l}\text { 1,1,1-TRICHLOROETHANE .0095X, ACETONE .0095X, CRES } \\
\text { ETHYL KETONE .0095X, METEYL ISOBUTYL KETONE .0095X } \\
.0095 \%\end{array}$ & $\begin{array}{l}\text { ACID .0095\%, METHYL } \\
\text { GYLENE CHLORIDE }\end{array}$ & $\begin{array}{l}\text { F001 F002 } \\
\text { F003 F004 } \\
\text { F005 WP02 }\end{array}$ & RD & 211 & $\mathbf{x}$ & & \\
\hline 2 & & 4289 & & & & 052094 & & $\mathbf{s}$ & I & $\begin{array}{l}\text { 1,1,1-TRICHLOROETHANE .0065X, ACETONE .0065X, CRES } \\
\text { PHTBLLATE (DOP) 2.615\%, METAYL ETEYL KETONE .0065X } \\
.0065 \% \text {, METHYLENE CHLORIDE .0065X }\end{array}$ & $\begin{array}{l}\text { ACID .0065\%, DIOCTYL } \\
\text { HYL ISOBUTYL KETONE }\end{array}$ & $\begin{array}{l}\text { F001 F002 } \\
\text { F003 F004 } \\
\text { F005 WC02 } \\
\text { WPO2 }\end{array}$ & RD & 26 & $\mathbf{x}$ & & \\
\hline 3 & & 4,4917 & & & & 092294 & & s & 0 & $\begin{array}{l}\text { 1,1,1-TRICELOROETHANE .0089\%, ACETONE .OOB9X, CRES } \\
\text { ETHYL KETONE .0089\%, METHYL ISOBUTYL KETONE .0089 } \\
.0089 \%\end{array}$ & $\begin{array}{l}\text { ACID .0089z, METHYL } \\
\text { HYLENE CBLORIDE }\end{array}$ & $\begin{array}{l}\text { F001 F002 } \\
\text { F003 F004 } \\
\text { F005 } \mathrm{WPO2}\end{array}$ & RD & 19 & $\mathbf{x}$ & & \\
\hline 4 & & .5354 & & & & 120894 & & $\mathbf{s}$ & ro & $\begin{array}{l}\text { 1,1,1-TRTCHLOROETHANE .009x, ACETONE .009x, CRESYL } \\
\text { ETHYL KETONE .009\%, METEYL ISOBUTYL KETONE .009x, }\end{array}$ & $\begin{array}{l}\text { ID .009x, METHYL } \\
\text { LENE CELORIDE .009X }\end{array}$ & $\begin{array}{l}\text { F001 F002 } \\
\text { F003 F004 } \\
\text { F005 WP02 }\end{array}$ & RD & 73 & $\mathbf{x}$ & & \\
\hline 3 & & 15437 & & & & 122294 & & $\mathbf{s}$ & I & $\begin{array}{l}1,1,1 \text { TRICHLOROETHANE .0104X, ACETONE .0104X, CRES } \\
\text { ETHY KETONE .0104X, METHXL ISOBUTYL KETONE .0104Z } \\
.0104 x\end{array}$ & $\begin{array}{l}\text { ACID .0104X, METHYL } \\
\text { HYLENE CHLORIDE }\end{array}$ & $\begin{array}{l}\text { F001 F002 } \\
\text { F003 F004 } \\
\text { F005 WPO2 }\end{array}$ & $\mathbf{R D}$ & 337 & $\mathbf{x}$ & & \\
\hline \multicolumn{18}{|c|}{$\begin{array}{l}\text { 16. CongenTS (Enter information by section and/or line number--See Page 13). } \\
\text { FACILITY: } 241 \mathrm{TX}\end{array}$} \\
\hline & & & & & & & & & & & & & & PAGE 100 & of 1 & & \\
\hline
\end{tabular}




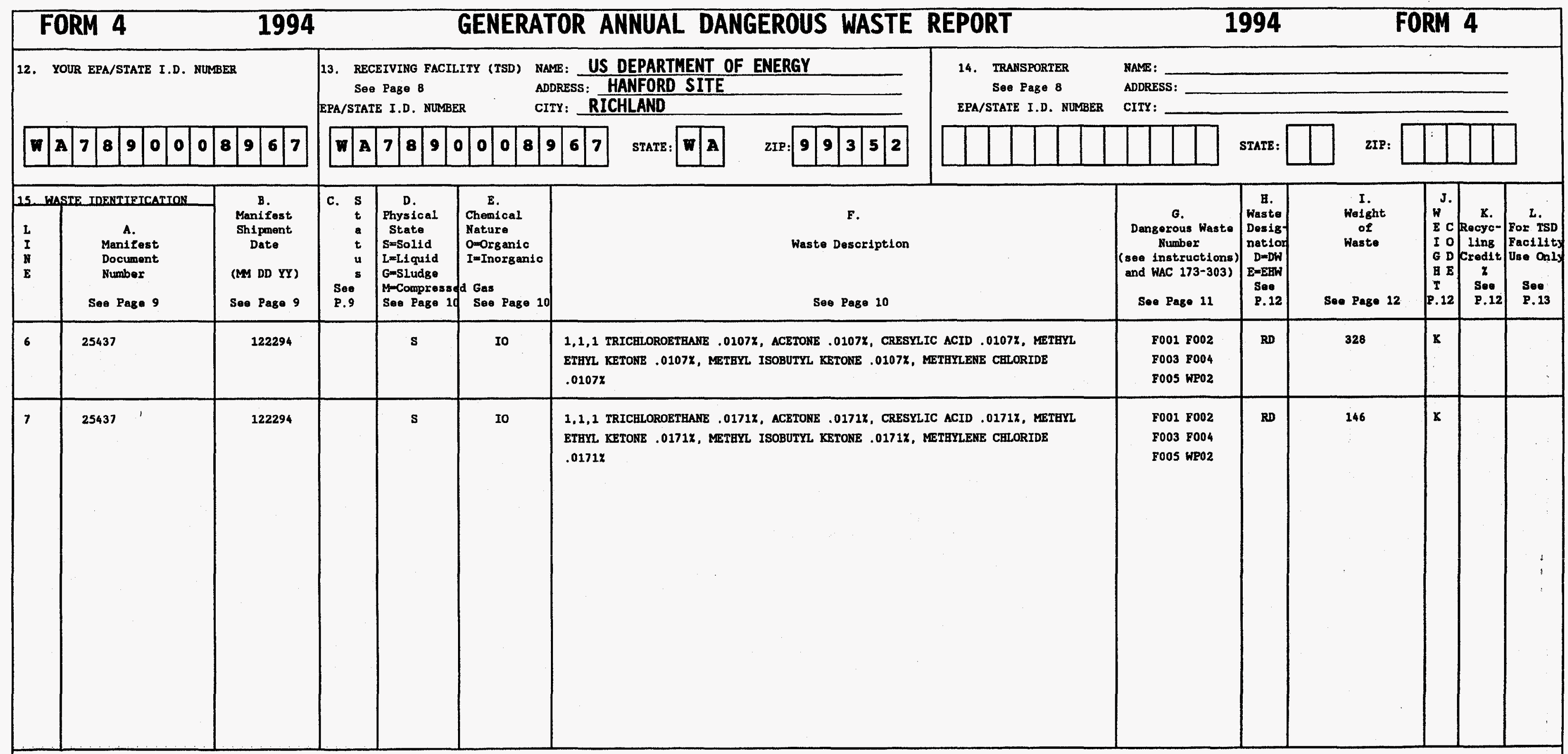

16. COMMENTS (Enter information by section and/or line number--See Page 13). FACILITY: 241TX

PAGE 101 OF 161 


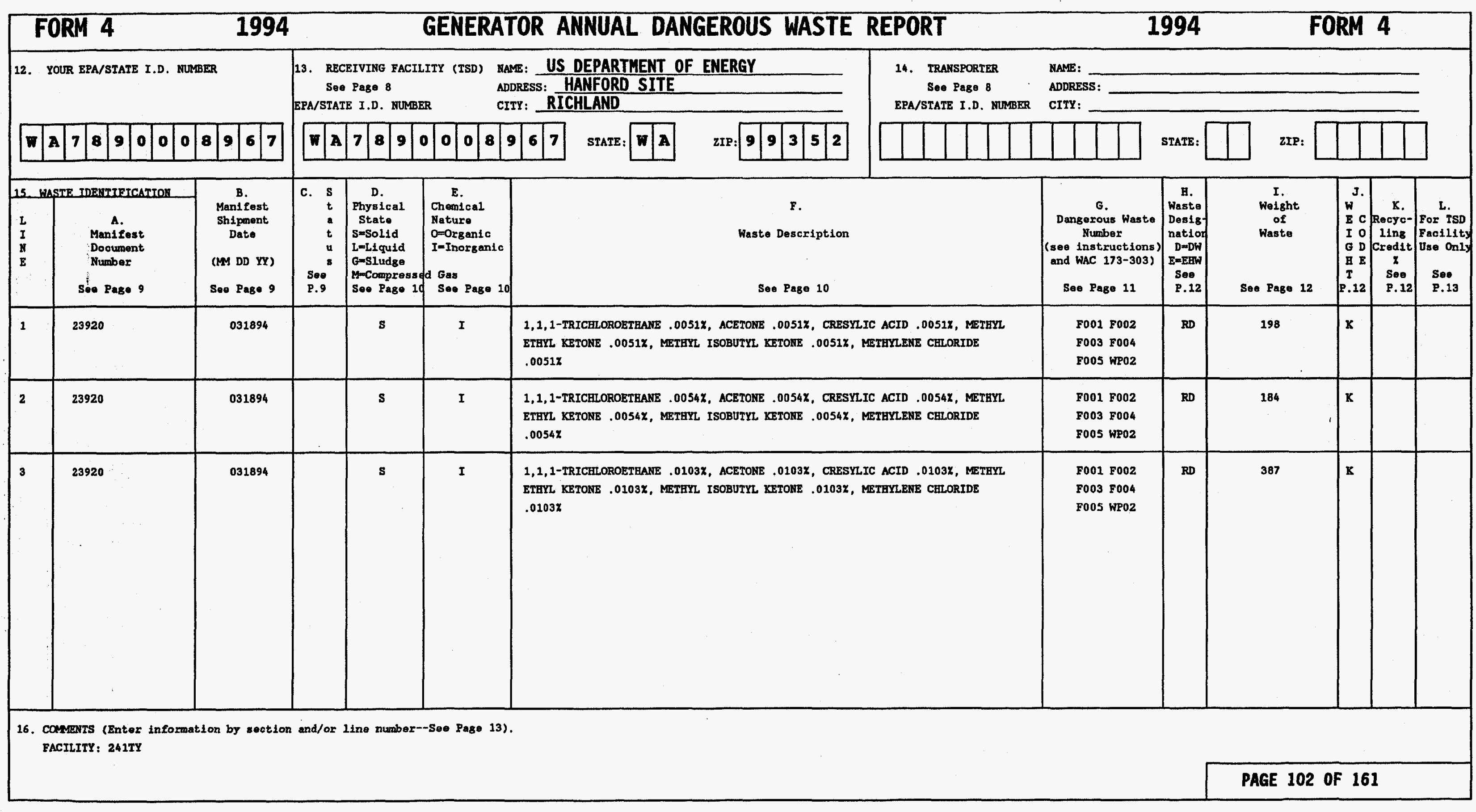




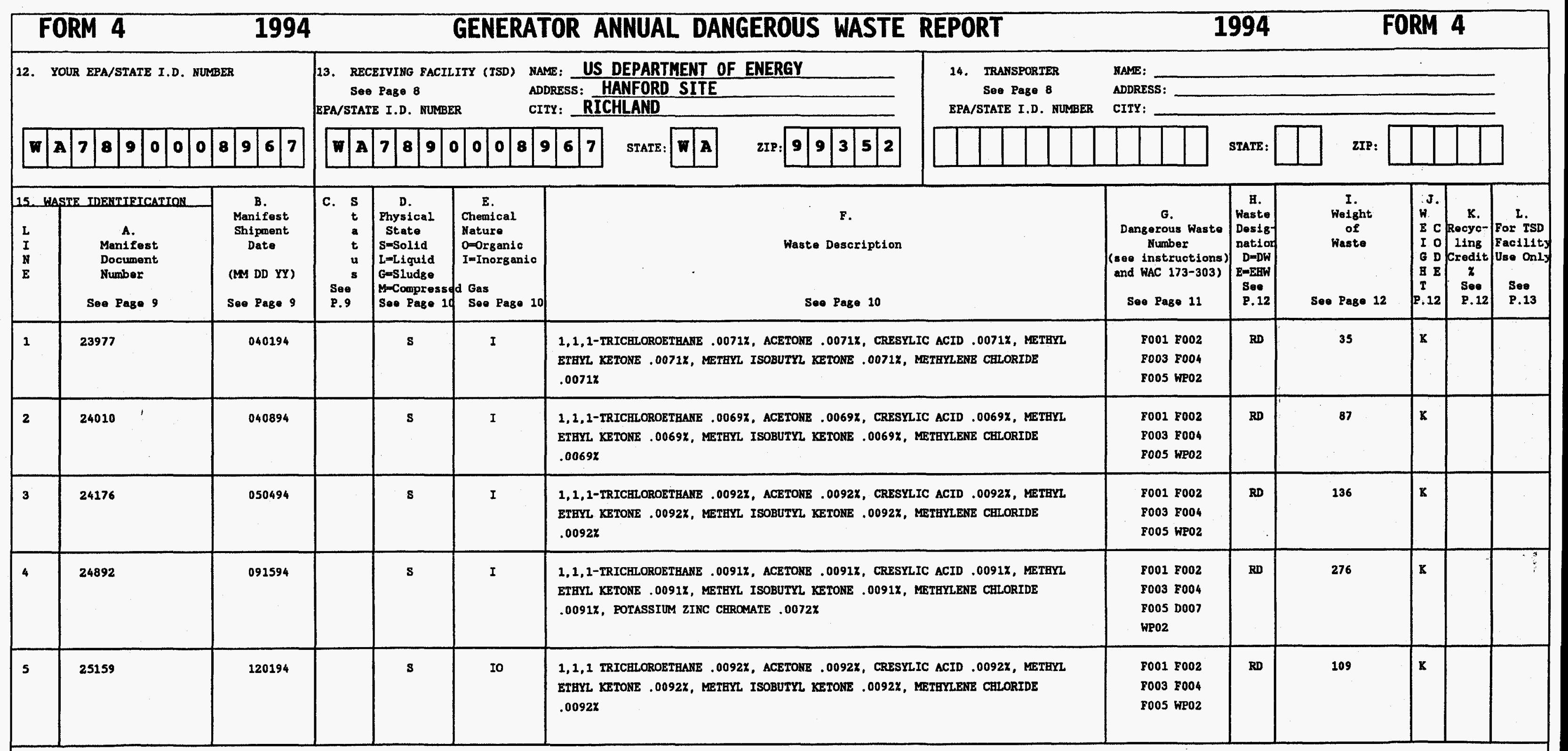

16. COMMENTS (Enter information by section and/or line number--Sae Page 13). FACILITY: 241U

PAGE 103 OF 161 


\begin{tabular}{|c|c|c|c|c|c|c|c|c|c|c|c|c|c|}
\hline & $\longdiv { R M 4 }$ & 1994 & & & GENERAT & OR ANNUAL DANGEROUS WASTE & EPORT & & $\overline{994}$ & & RM & & \\
\hline & OUR EPA/STAIE I.D. & & $\begin{array}{l}\text { 13. } \mathrm{REC} \\
\text { Se } \\
\text { EPA/STA }\end{array}$ & $\begin{array}{l}\text { EEIVING FACIL } \\
\text { Page } 8 \\
\text { CE I.D. NUMBE }\end{array}$ & $\begin{array}{ll}\text { CITY (TSD) } & \text { NAM } \\
\text { ER } & \text { ADD } \\
\text { CIT }\end{array}$ & 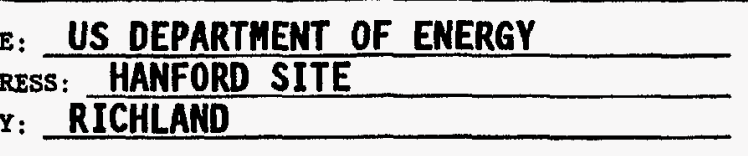 & $\begin{array}{l}\text { 14. TRANSFORTER } \\
\text { See Page 8 } \\
\text { EPA/STATE I.D. MUMBER }\end{array}$ & $\begin{array}{l}\text { NAME: } \\
\text { ADDRESS: } \\
\text { CITY: }\end{array}$ & & 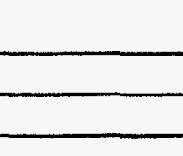 & & - & \\
\hline$w$ & \begin{tabular}{|l|l|l|l|l|l|} 
& 8 & 9 & 0 & 0 \\
\end{tabular} & \begin{tabular}{l|l|l|l|}
8 & 9 & 6 & 7
\end{tabular} & 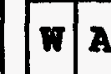 & \begin{tabular}{l|l|l|l}
7 & 8 & 9 & 0 \\
\end{tabular} & \begin{tabular}{l|l|l|l|l}
0 & 0 & 0 & 8 & 9
\end{tabular} & 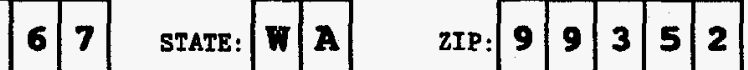 & & & sTATE: & 2IP: & & & \\
\hline 25 & STE IDERTIETCATTON & & c. $s$ & & & & & & B. & I. & J. & $x$ & \\
\hline $\begin{array}{l}\text { L } \\
\text { I } \\
\text { E } \\
\text { E }\end{array}$ & $\begin{array}{l}\text { A. } \\
\text { Manifest } \\
\text { Document } \\
\text { Number } \\
\text { Sel Page 9 }\end{array}$ & $\begin{array}{c}\begin{array}{c}\text { Shipment } \\
\text { Date }\end{array} \\
\text { (MM DD YY) } \\
\text { See Page 9 }\end{array}$ & $\begin{array}{r}\mathrm{a} \\
\mathrm{t} \\
\mathrm{u} \\
\mathrm{s} \\
\text { So.9 } \\
\mathrm{P.9}\end{array}$ & 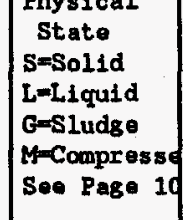 & $\begin{array}{l}\text { Nature } \\
\text { omorganic } \\
\text { I=Inorganic } \\
\text { das } \\
\text { SeO Page } 10\end{array}$ & $\begin{array}{l}\text { Waste Description } \\
\text { See Page } 10\end{array}$ & & $\begin{array}{c}\text { Dangerous Waste } \\
\text { Number } \\
\text { (see 1nstruotions) } \\
\text { and WAC 173-303) } \\
\text { See Page } 11\end{array}$ & 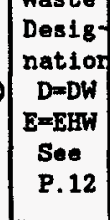 & $\begin{array}{c}\text { Waste } \\
\text { of } \\
\text { See Page } 12\end{array}$ & $\mid \begin{array}{ll}E & C \\
\text { I } & 0 \\
G & D \\
\text { B } & E \\
T \\
F .12\end{array}$ & 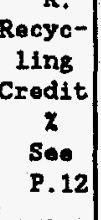 & $\begin{array}{c}\text { For TsD } \\
\text { Factits } \\
\text { Use onl } \\
\text { Soo } \\
\text { P.13 }\end{array}$ \\
\hline 6 & 25212 & 111094 & & s & I & $\begin{array}{l}\text { 1,1,1-TRICHLOROETHANE .0072x, ACETONE .0072X, CRES } \\
\text { ETRY KETONE .0072X, MEREYL ISOBUTYL KETONE .0072X } \\
.0072 x\end{array}$ & $\begin{array}{l}\text { ACID .0072x, METHYL } \\
\text { HYLENE CELORIDE }\end{array}$ & $\begin{array}{l}\text { F001 F002 } \\
\text { F003 F004 } \\
\text { F005 WP02 }\end{array}$ & $\mathrm{BD}$ & 23 & $\mathrm{k}$ & & \\
\hline 7 & 25417 & 121594 & & s & Io & $\begin{array}{l}\text { 1,1,1 TRICRLOROETHANE .0052x, ACETONE .0052X, CRES } \\
\text { PHTHALATE 2. B13z, METHYL, ETHYL KETONE .0052z, METH } \\
\text {.0052X, METHYLENE CHLORIDE .005ZZ }\end{array}$ & $\begin{array}{l}\text { ACID .00S2\%, DIOCTYL } \\
\text { OBUTYL KETONE }\end{array}$ & $\begin{array}{l}\text { F001 F002 } \\
\text { F003 F004 } \\
\text { F005 WC02 } \\
\text { WP02 }\end{array}$ & RD & 32 & $\mathrm{x}$ & & \\
\hline 8 & 25417 & 121594 & & $\mathbf{s}$ & Io & 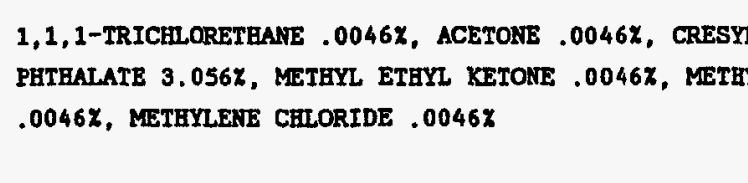 & $\begin{array}{l}\text { CID .0046\%, DIOCTYL } \\
\text { OBUTY KETONE }\end{array}$ & $\begin{array}{l}\text { F001 F002 } \\
\text { F003 F004 } \\
\text { F005 WC02 } \\
\text { WP02 }\end{array}$ & RD & 36 & $\mathbf{x}$ & & \\
\hline & $\begin{array}{l}\text { ITS (Entor in } \\
\text { ITY: 2410 }\end{array}$ & by soct & & number & - Page 1 & & & & & & & & \\
\hline & & & & & & & & & & PAGE 104 & $\mathbf{F} 16$ & & \\
\hline
\end{tabular}




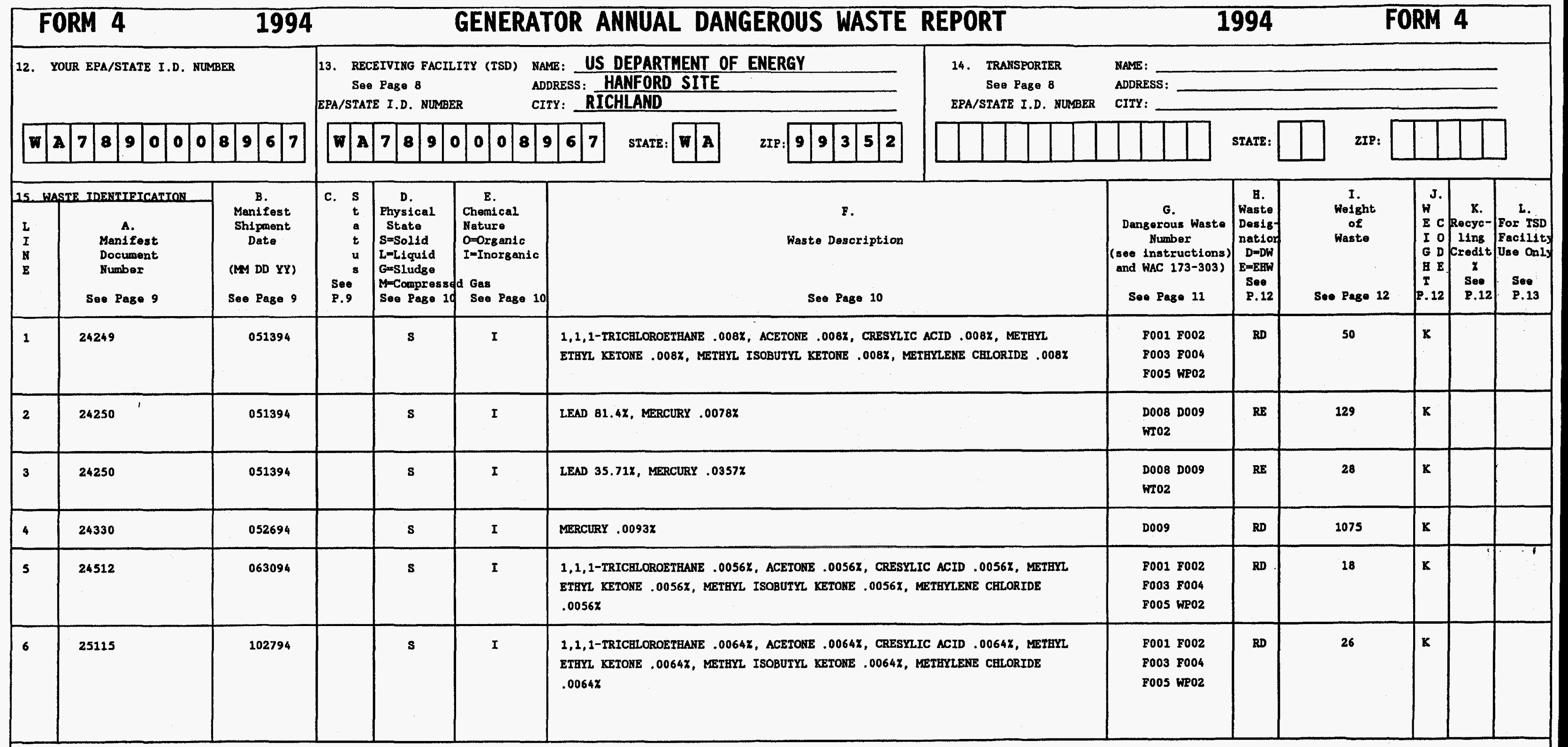

16. COMNENTS (Enter information by section and/or 1ine number--See Page 13). FACILITY: $242 \mathrm{~A}$ 


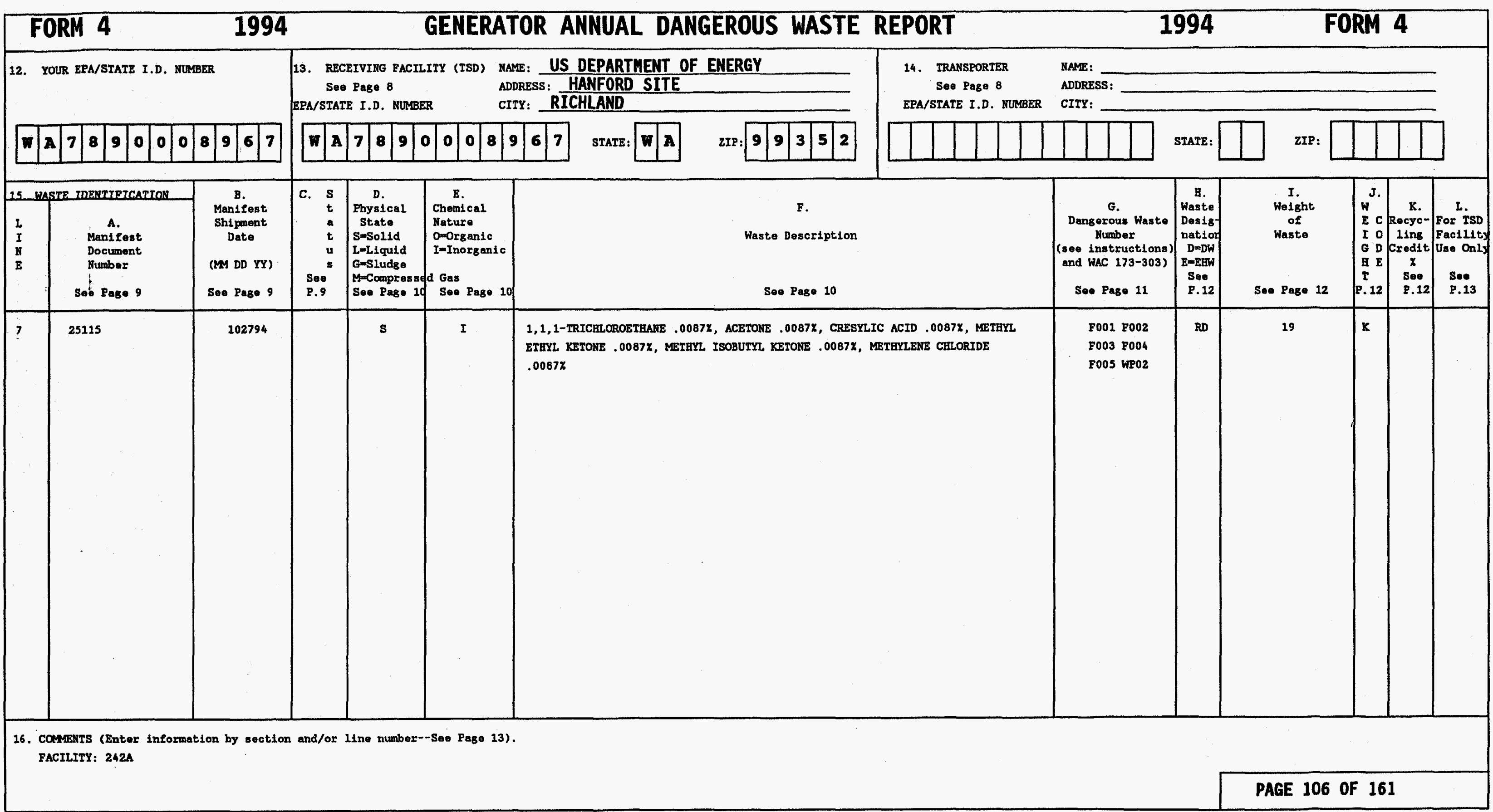




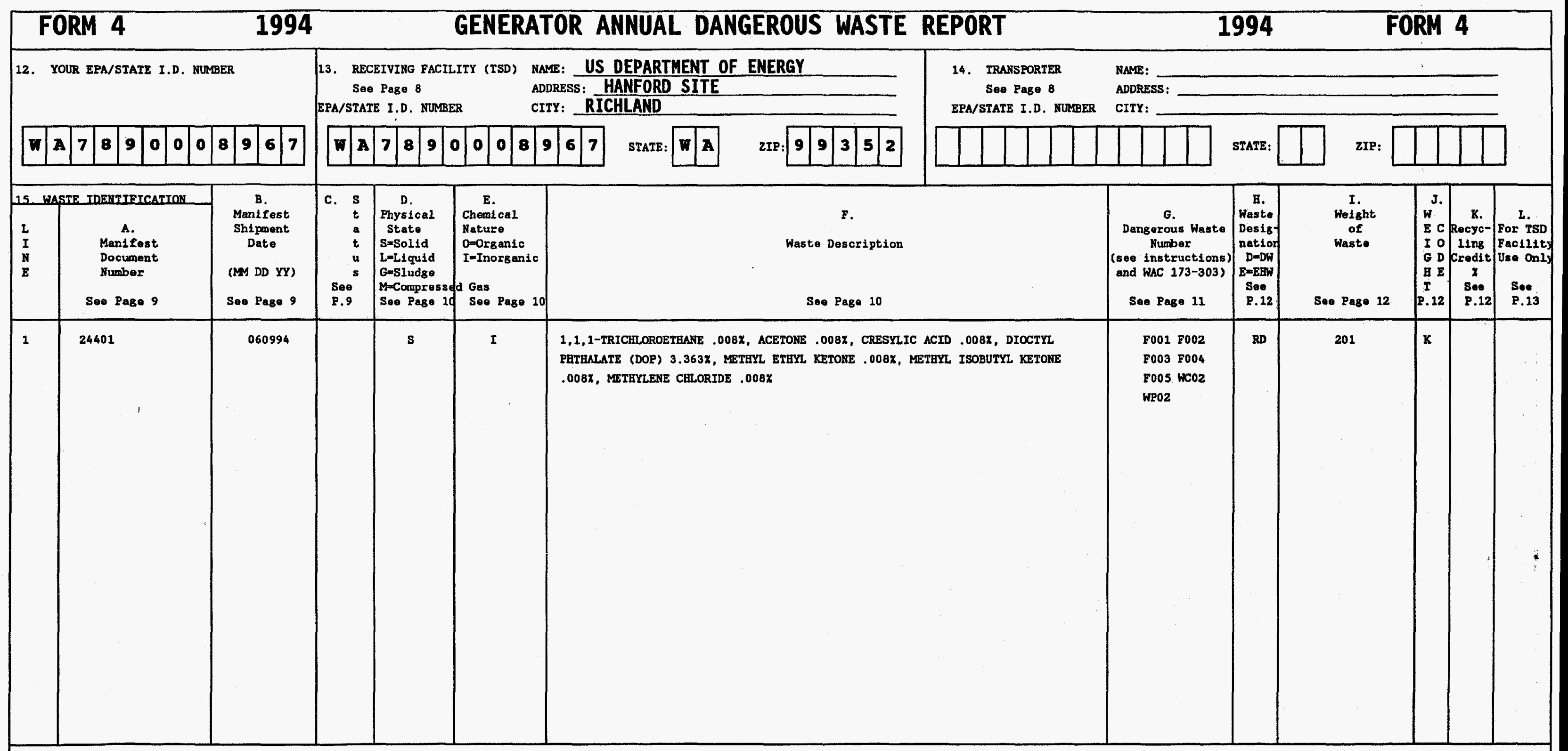

16. Comererss (Enter information by saction and/or 11ne number--See Page 13). FACIIITY: 243G 


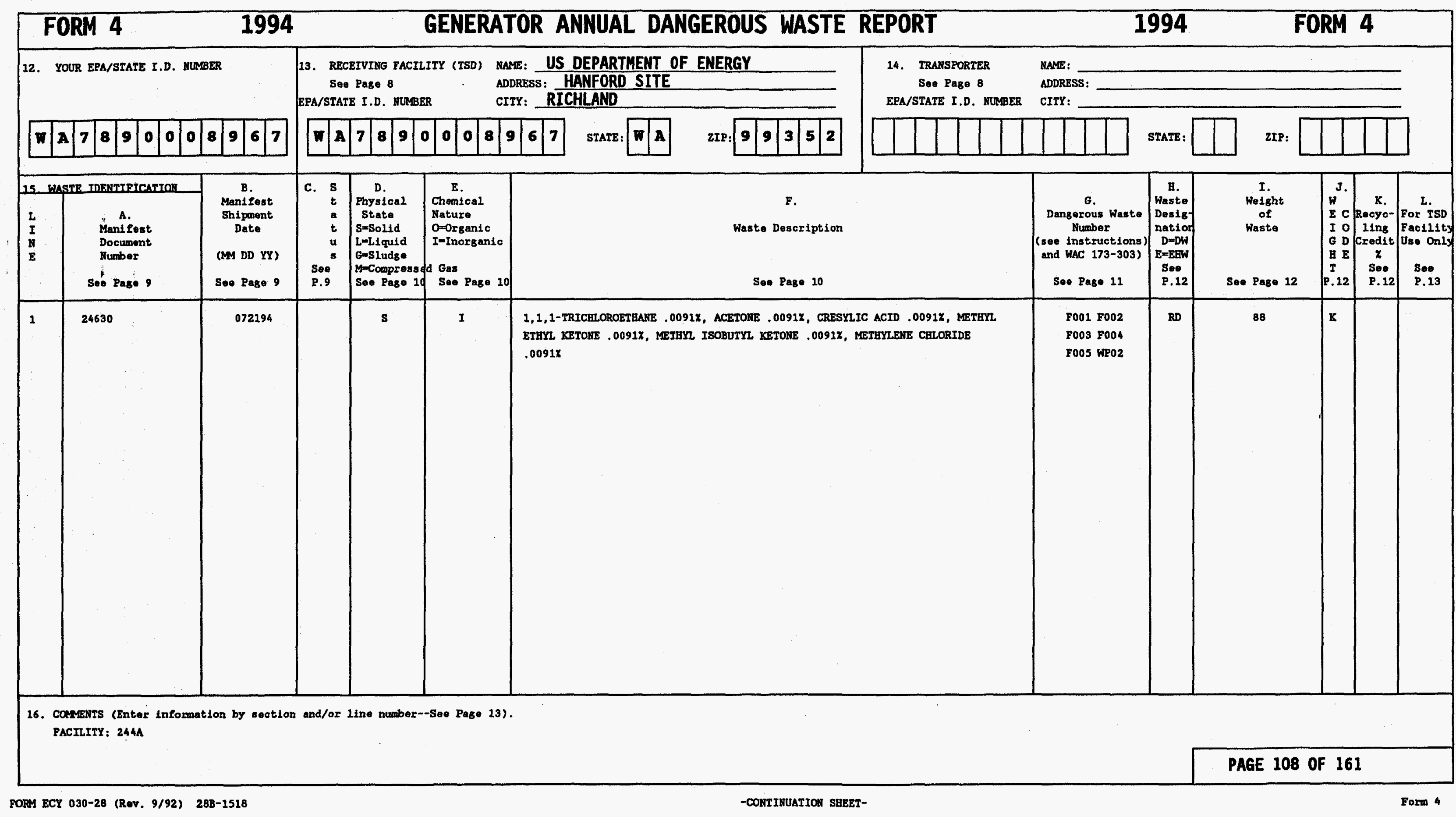




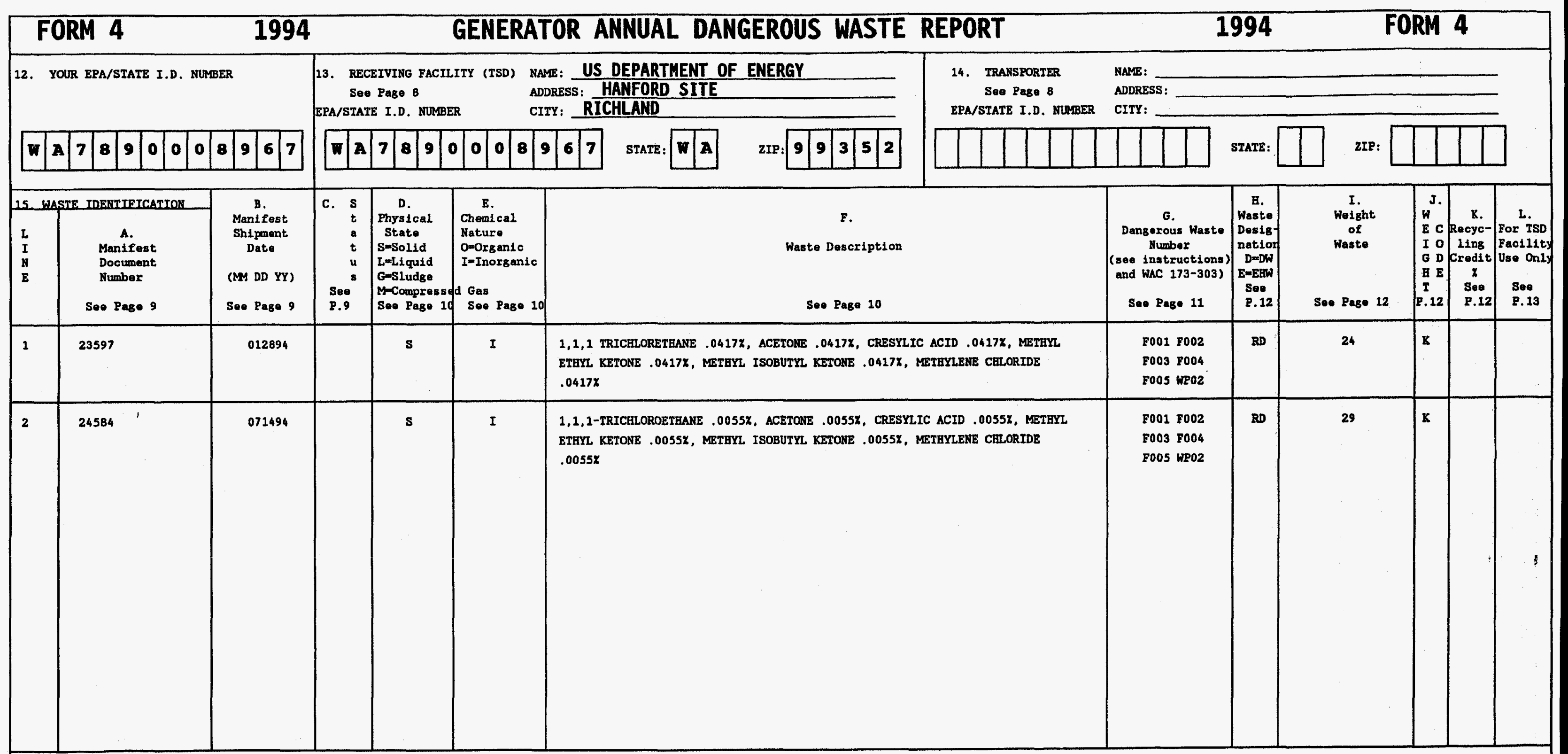

16. COMENTS (Enter information by section and/or line number--See Page 13) FACILITY: $244 \mathrm{BX}$

PAGE 109 OF 161 


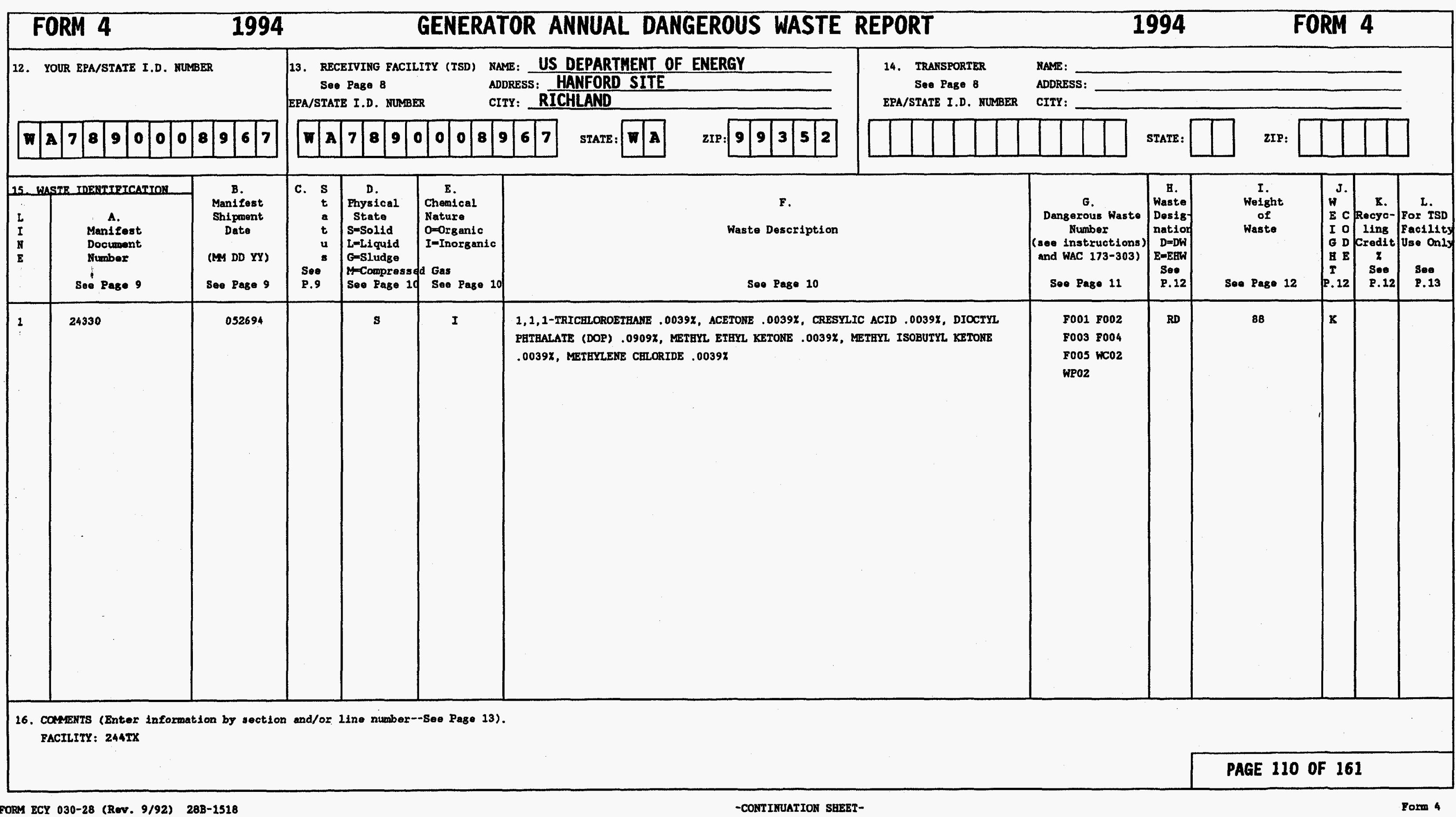




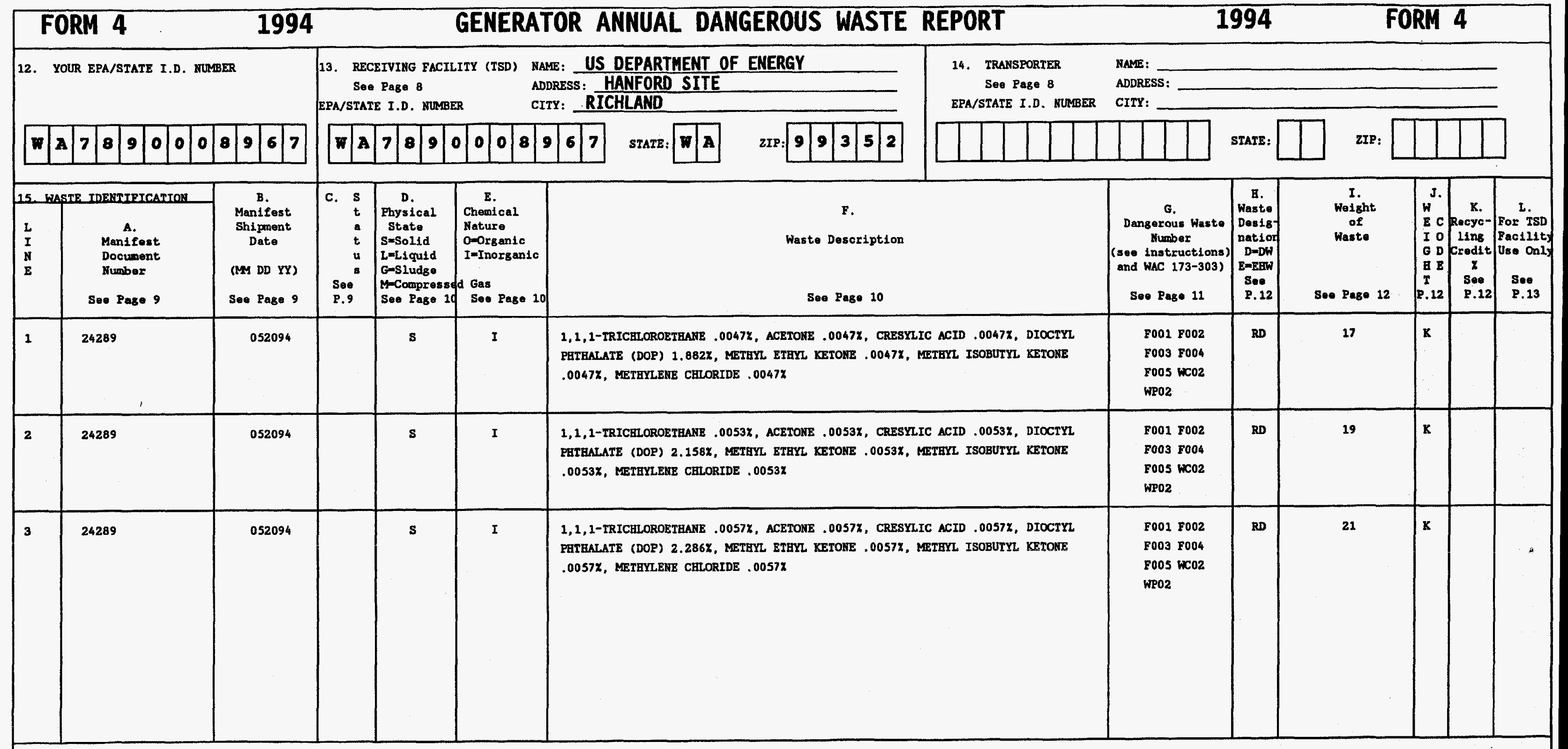

16. COMENIS (Enter information by section and/or 1ine number--See Page 13), FACILITY: 244U

PAGE 111 OF 161 


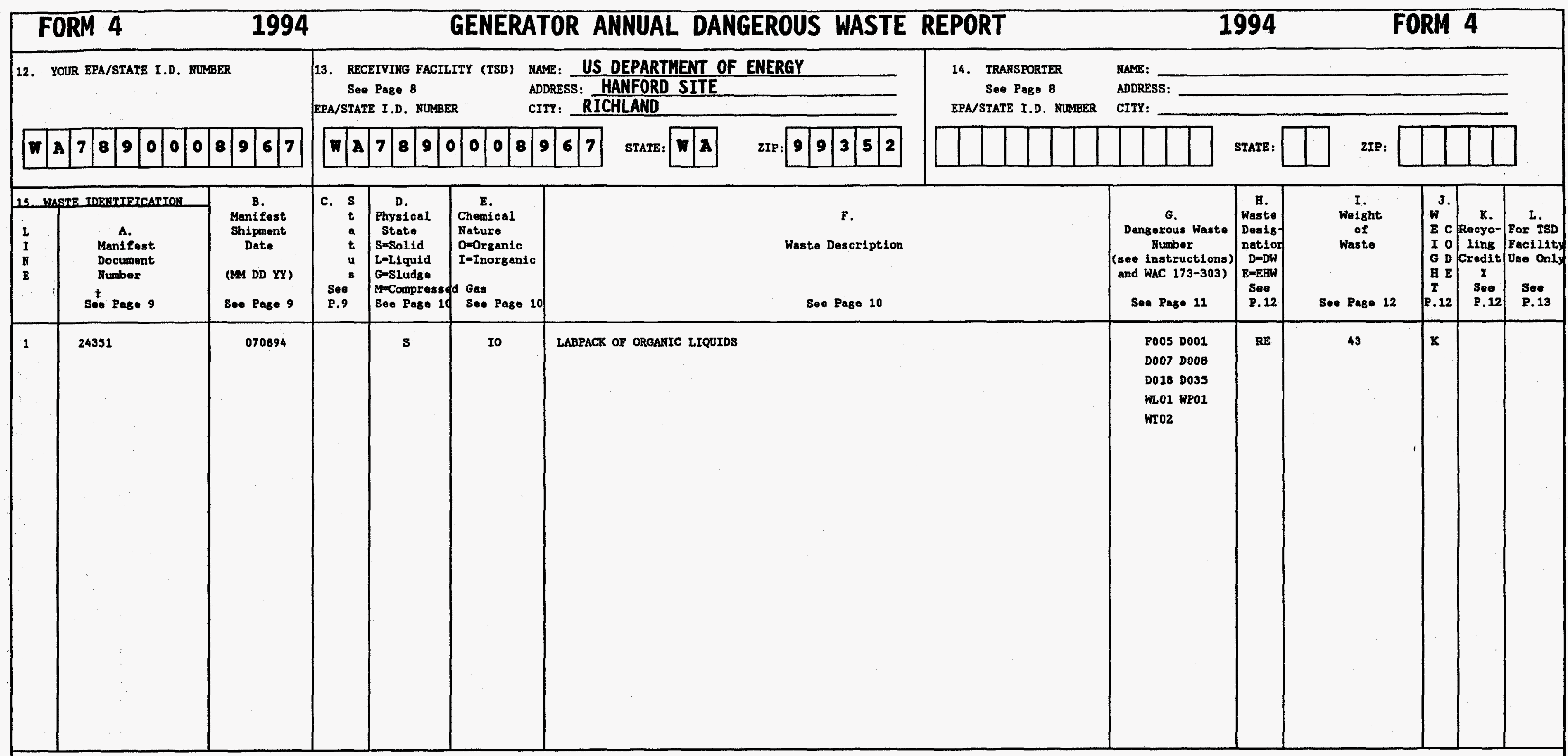

16. COMMENTS (Enter information by section and/or line number--See Page 13). FACILITY: 244UR 


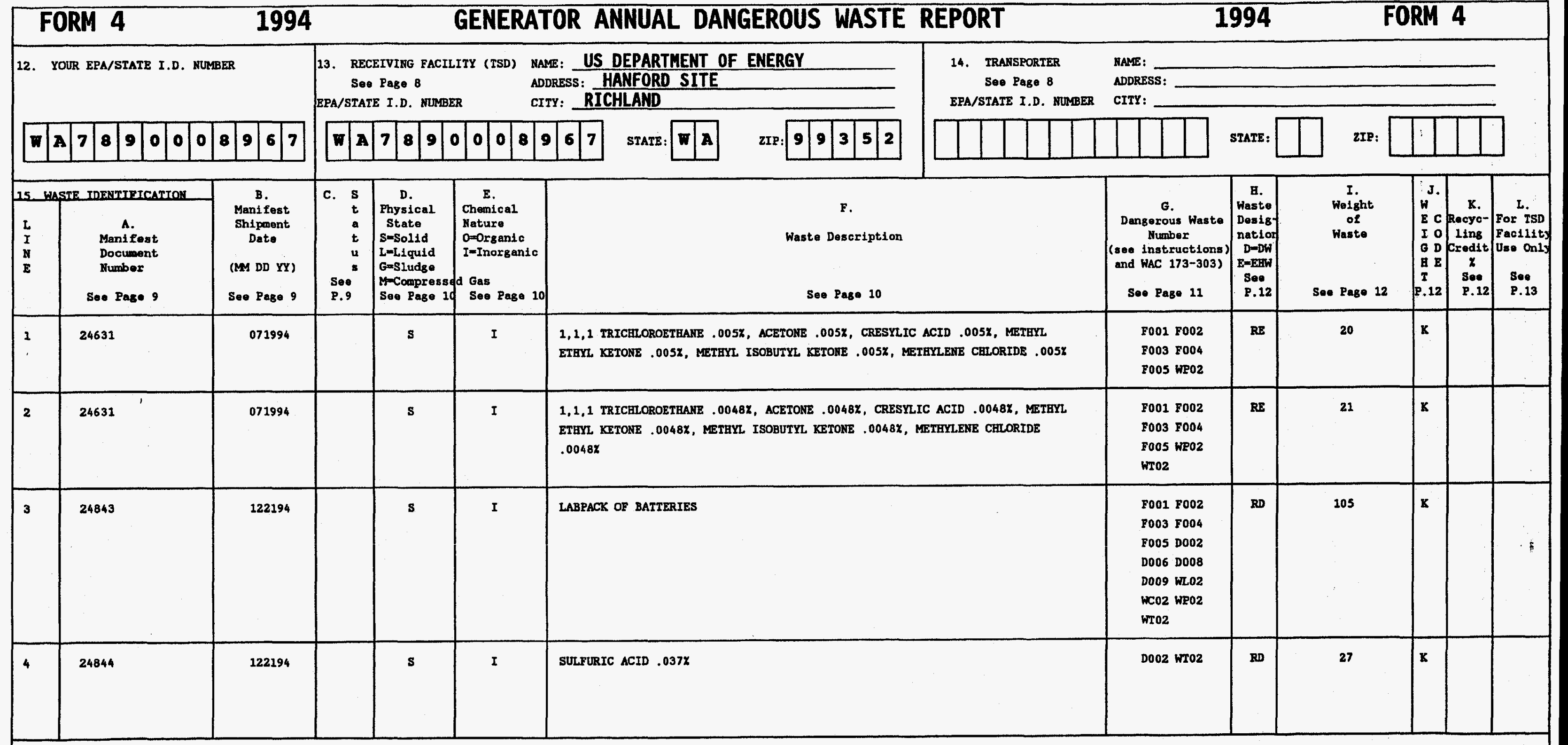

16. COMENTS (Enter Information by section and/or 1ine number--See Page 13). FACILITY: 2706 T

PAGE 113 OF 161 


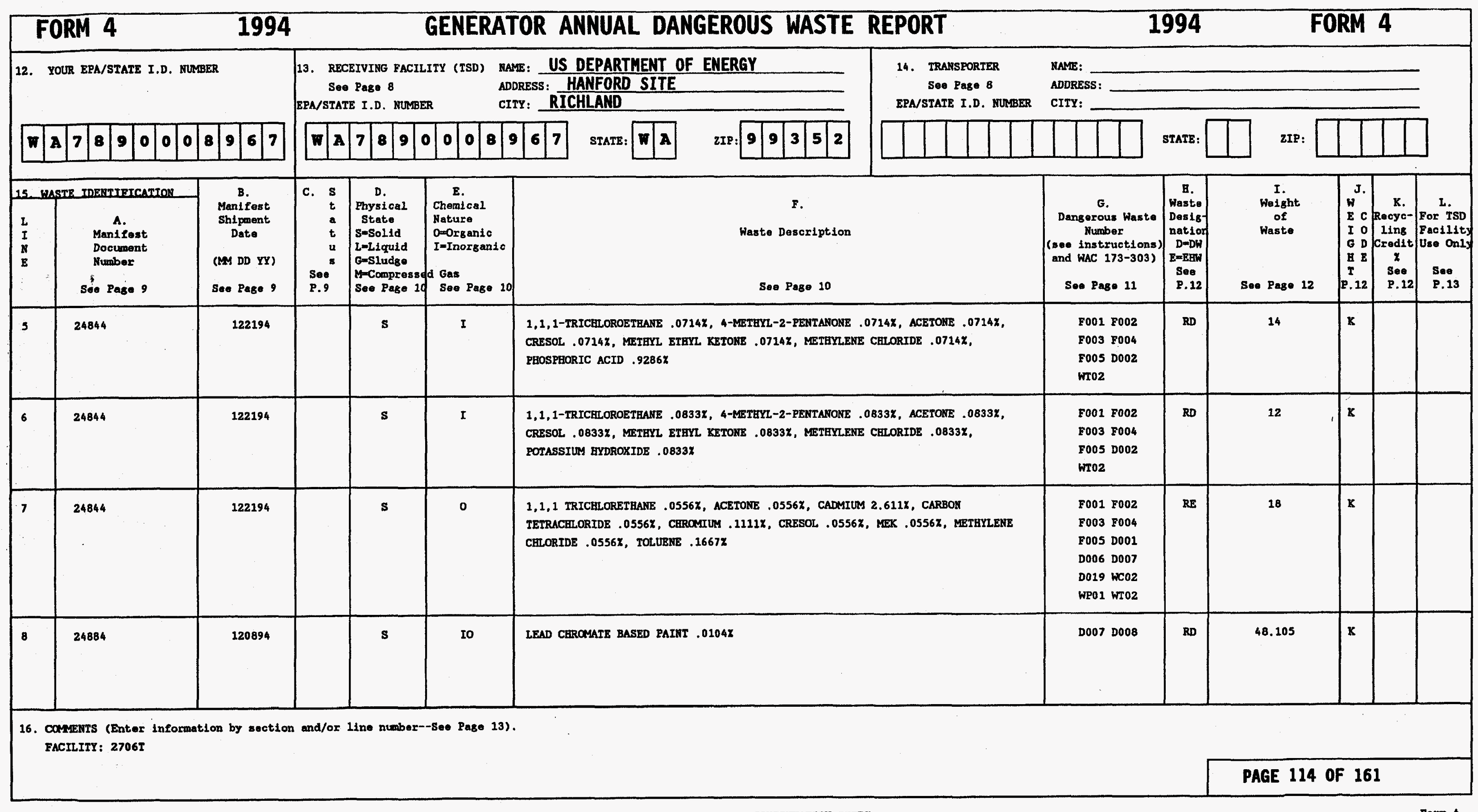




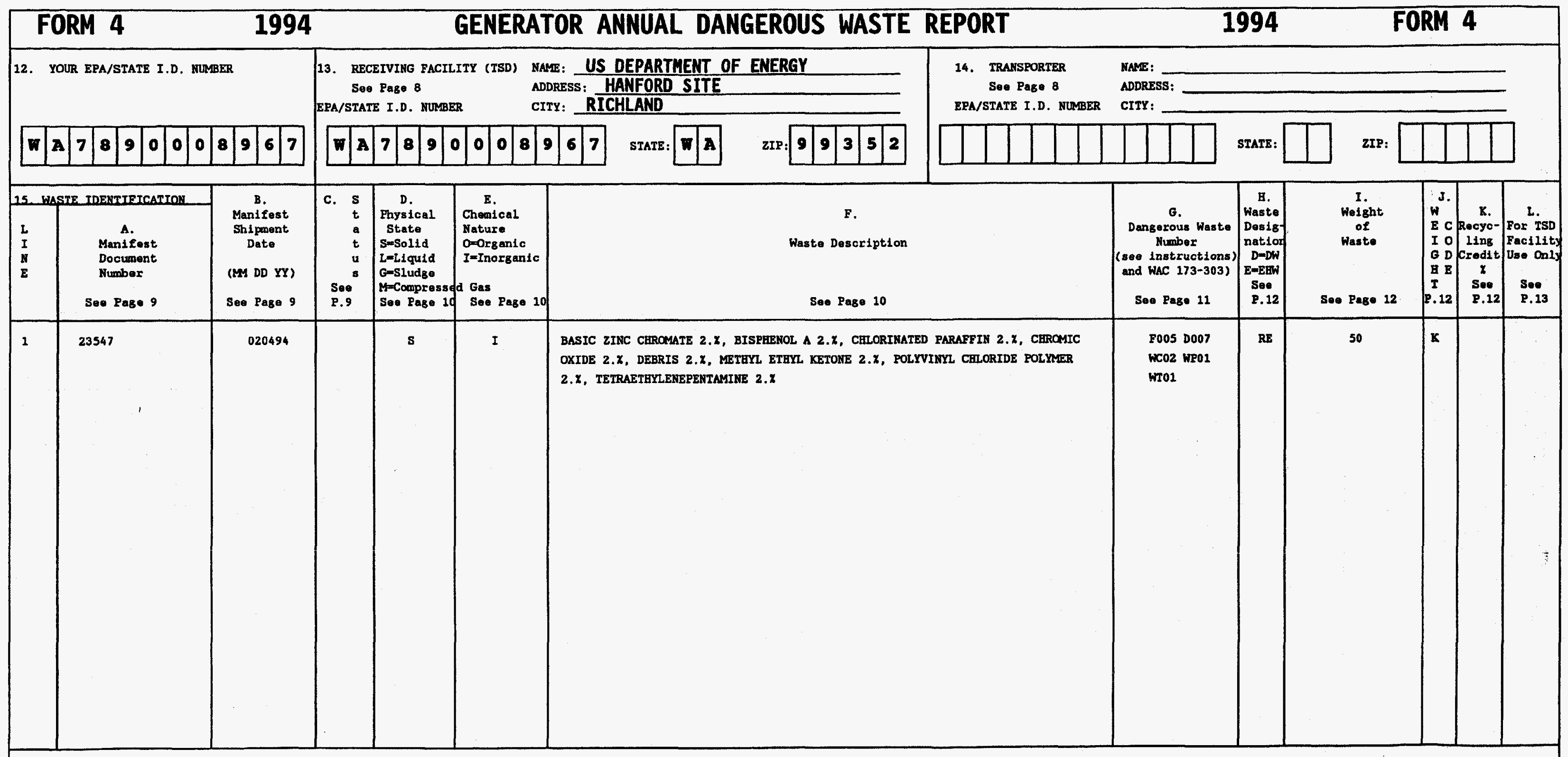

16. Comerrs (Enter inforwation by section and/or 1ine number--See Page is). FACILITY: 271CR 


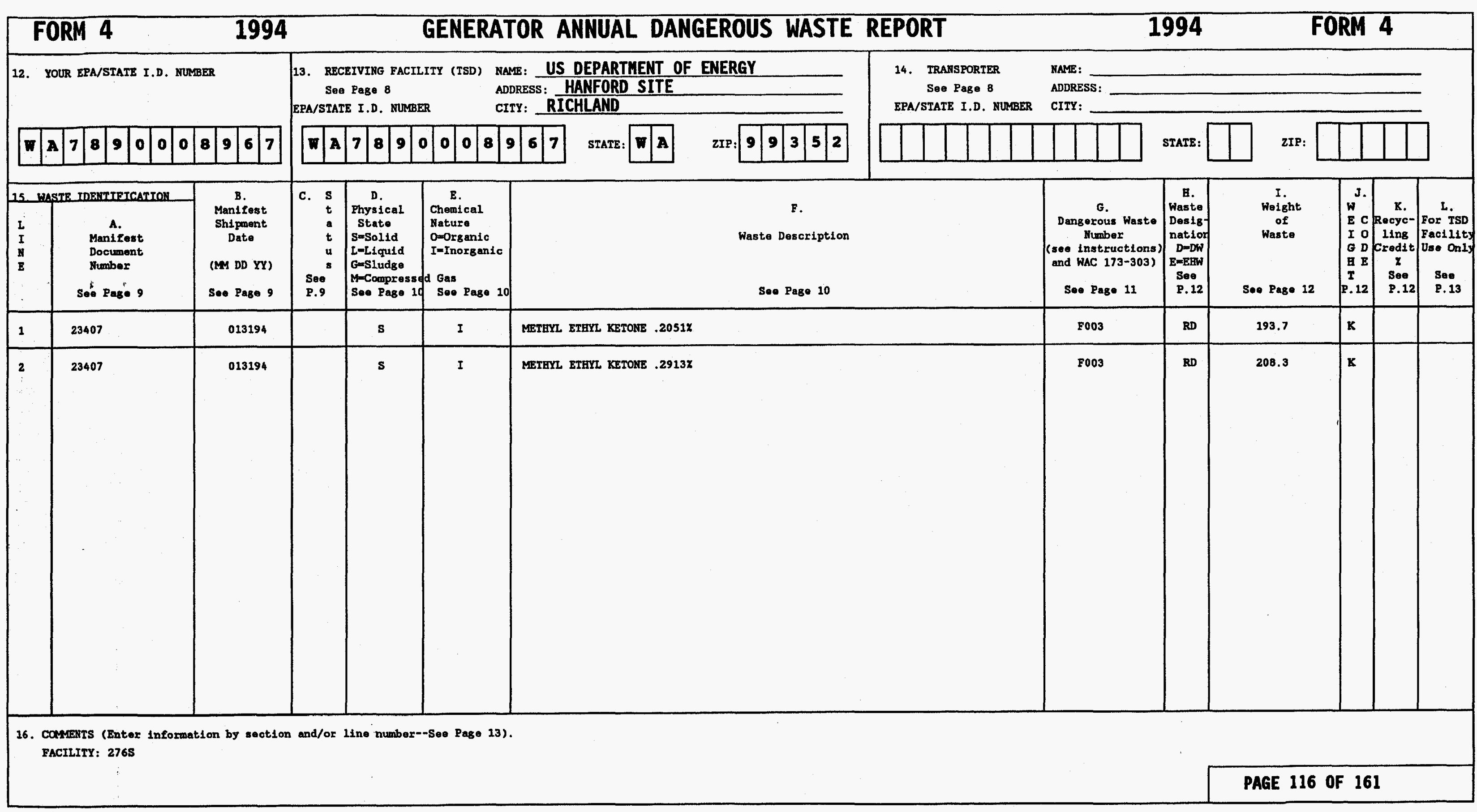




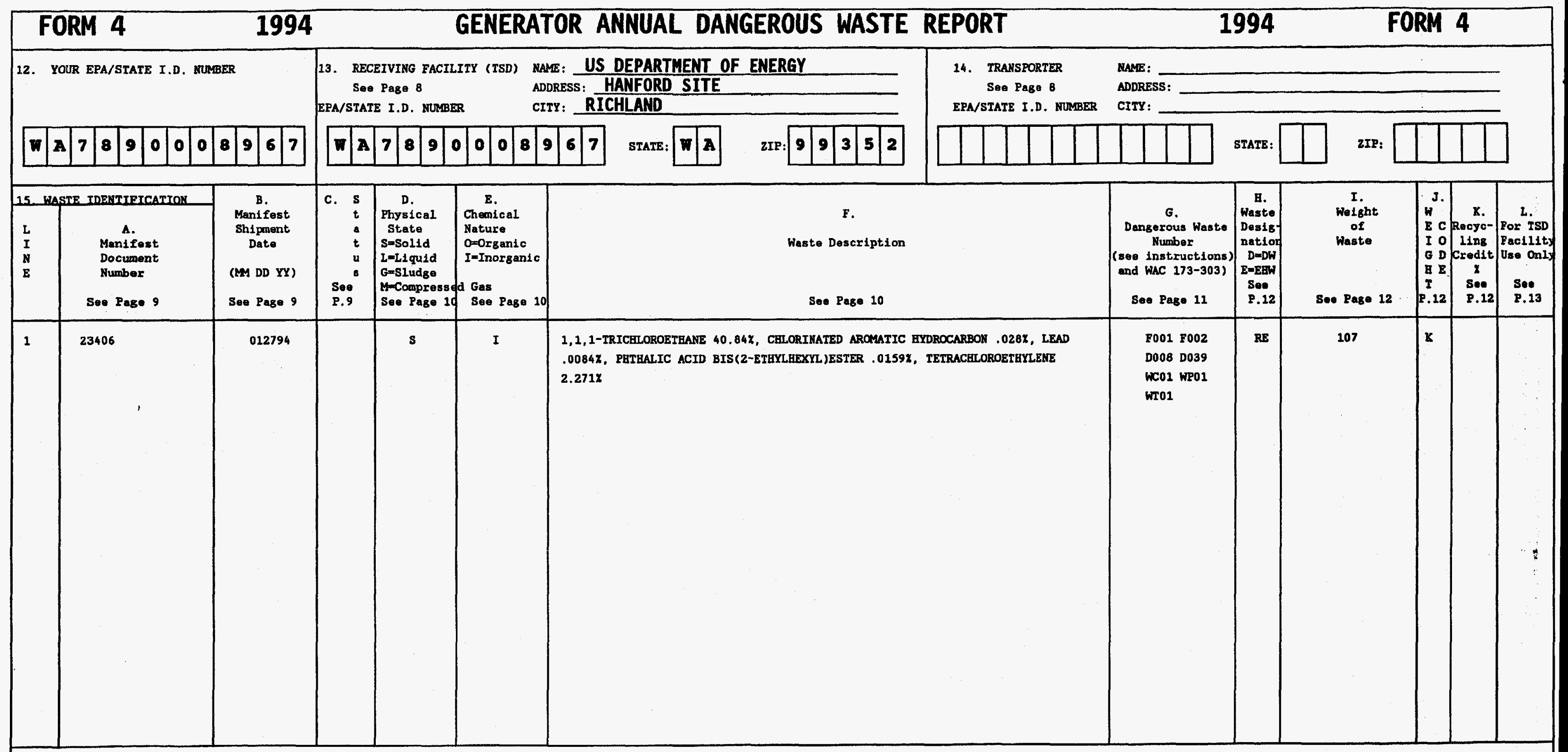

16. Camgars (Enter Information by section and/or line number--See Page 13), FACILITY: 303K 


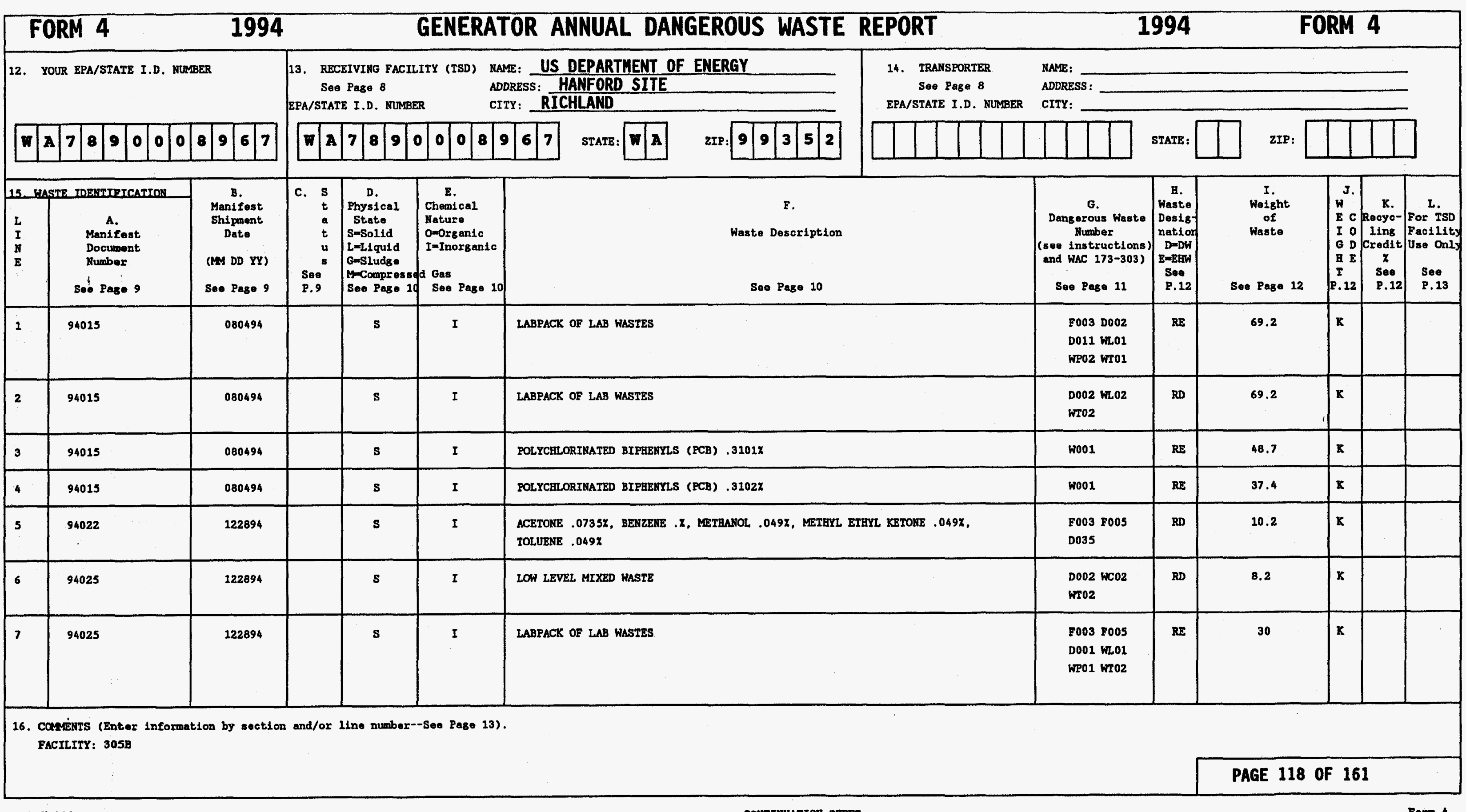




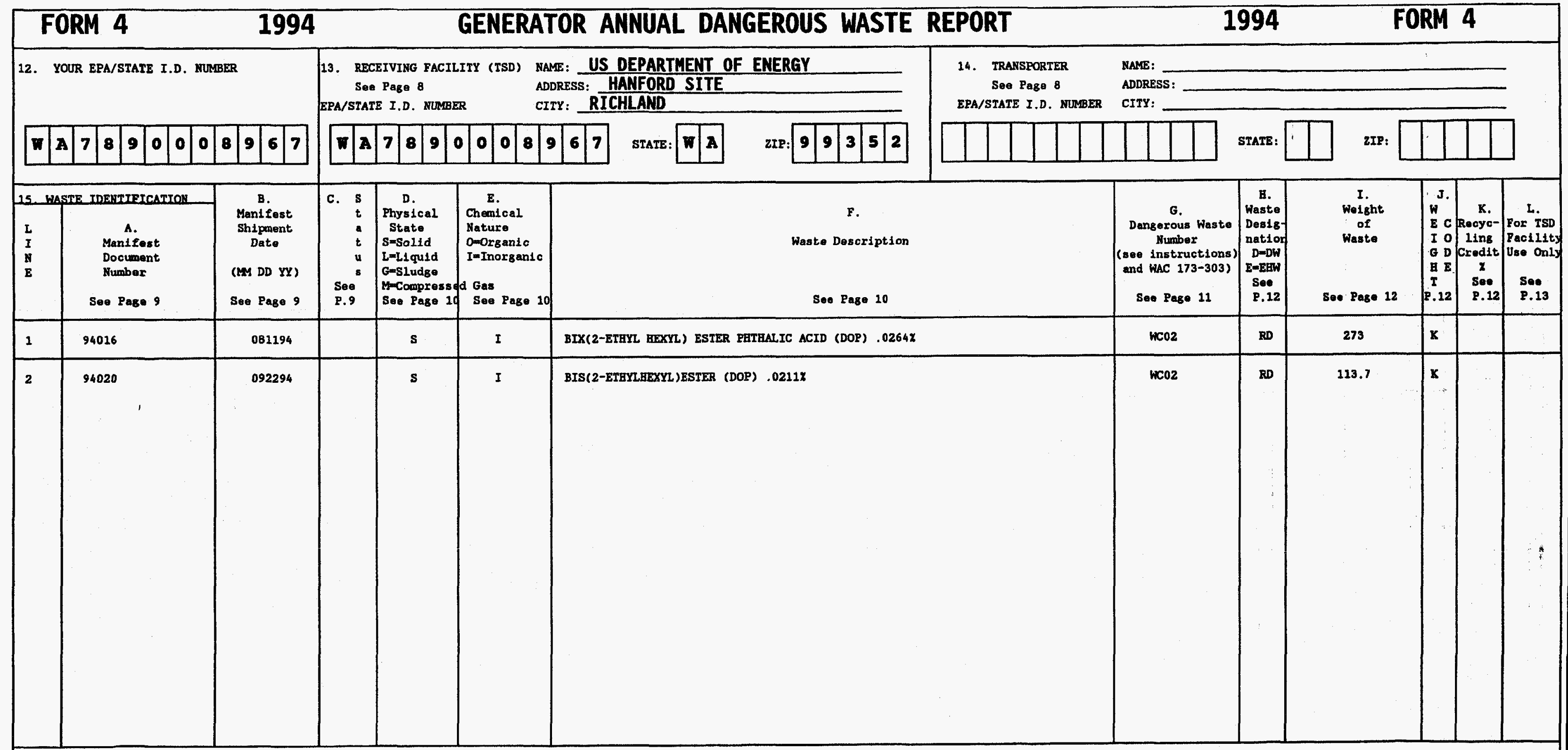

16. Cameris (Enter information by zection and/or 1ine number---See Page 13). PACILITY: 306 


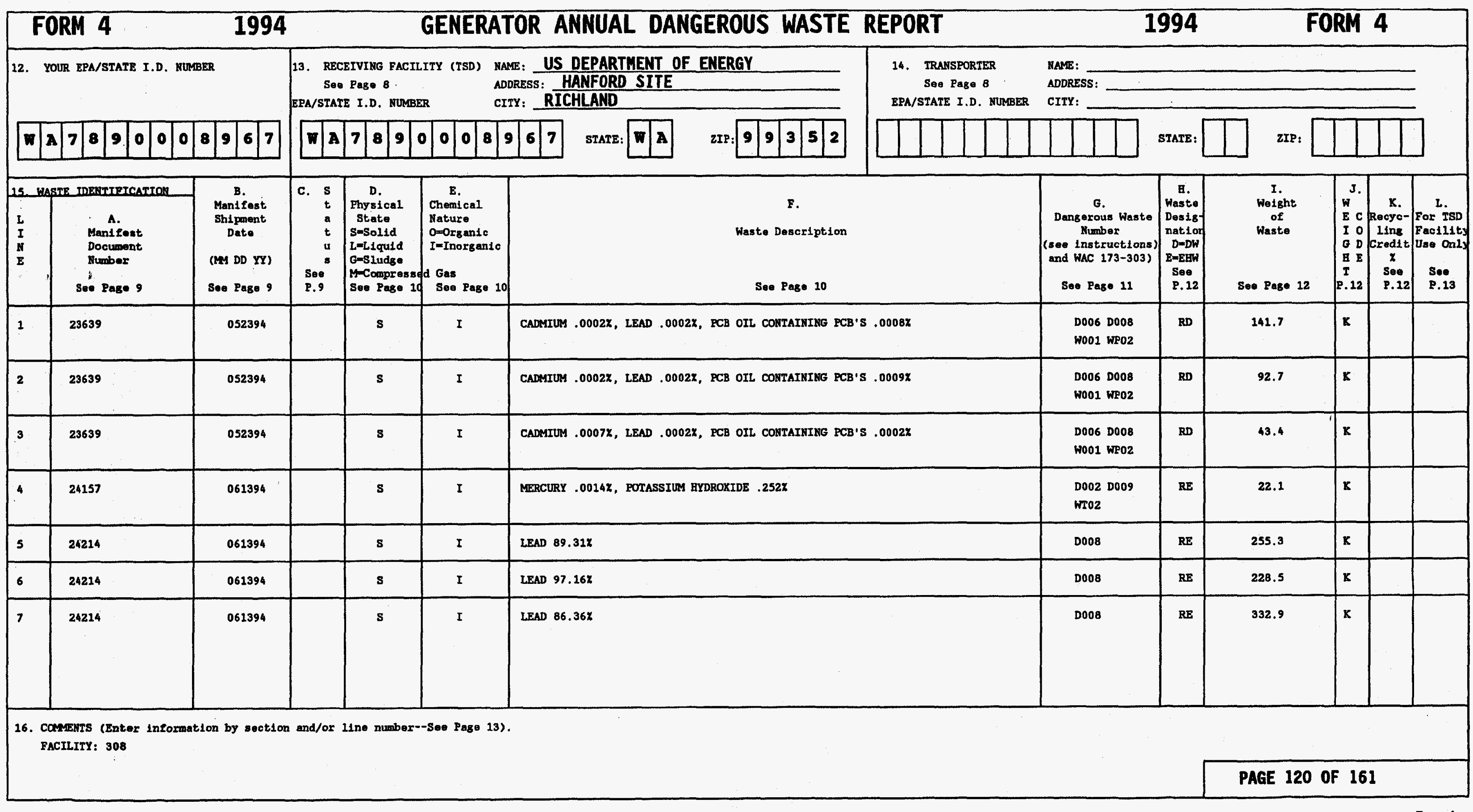




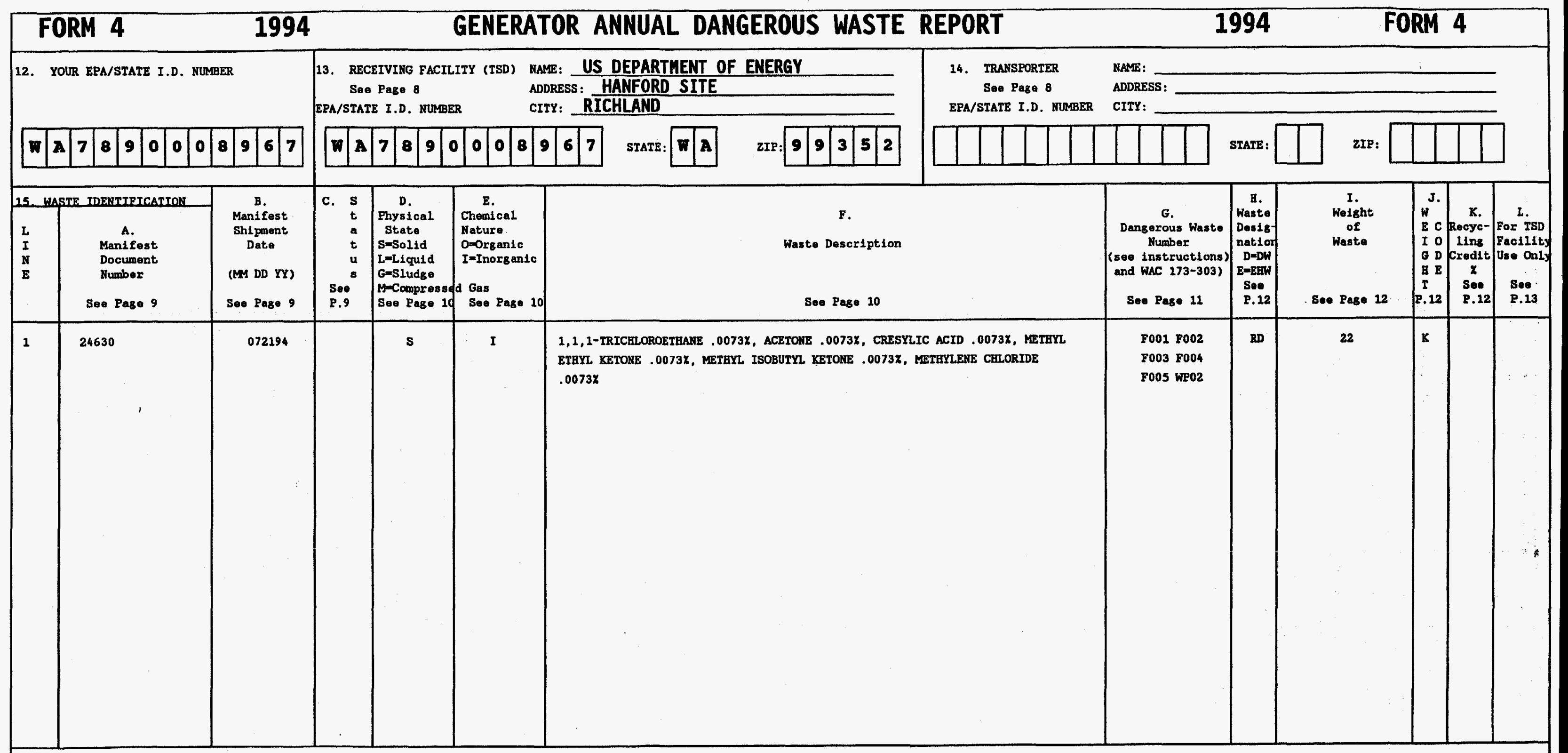

16. Comenrs (Entex information by section and/or 1ine number--See Page 13). FACILITY: 311 


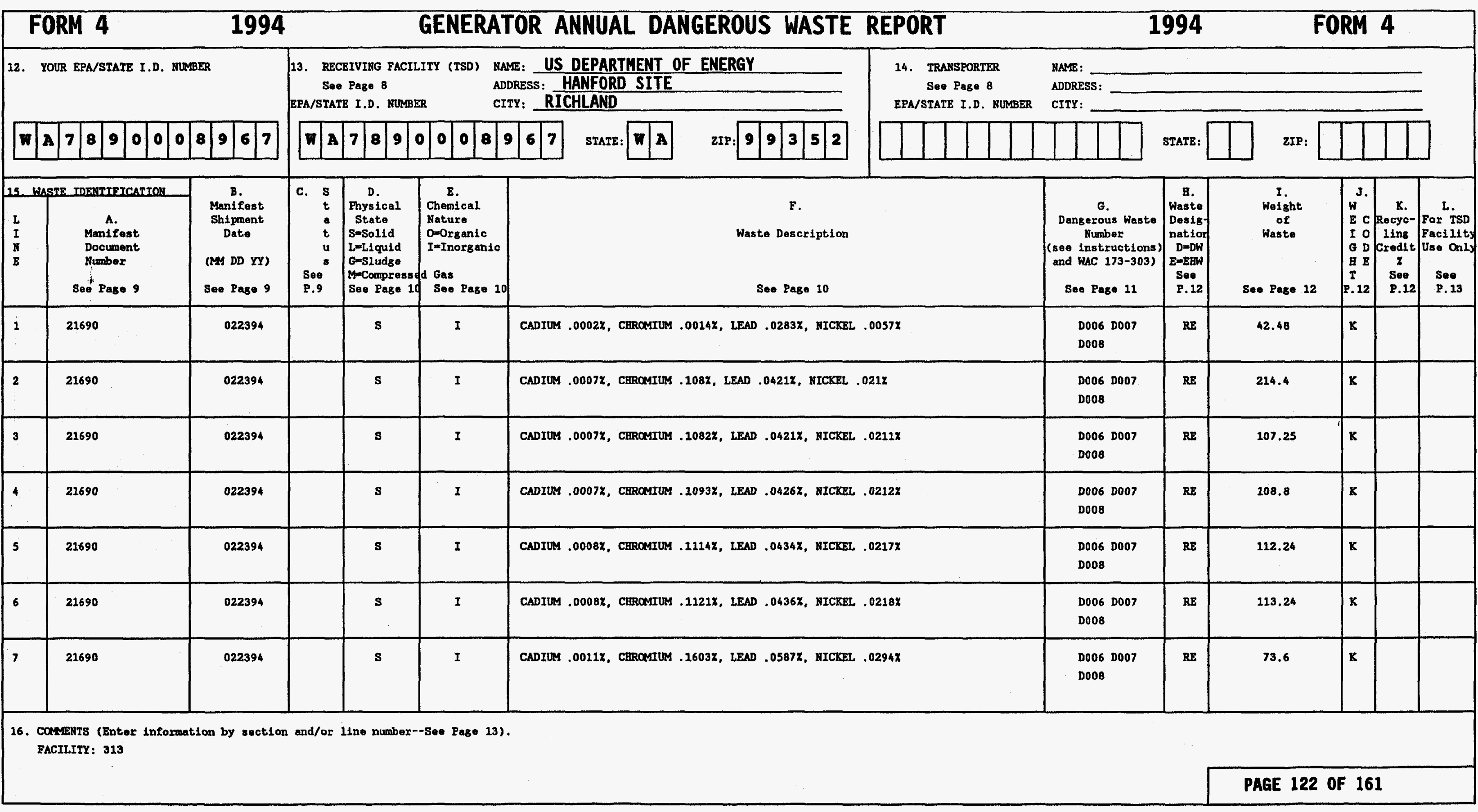




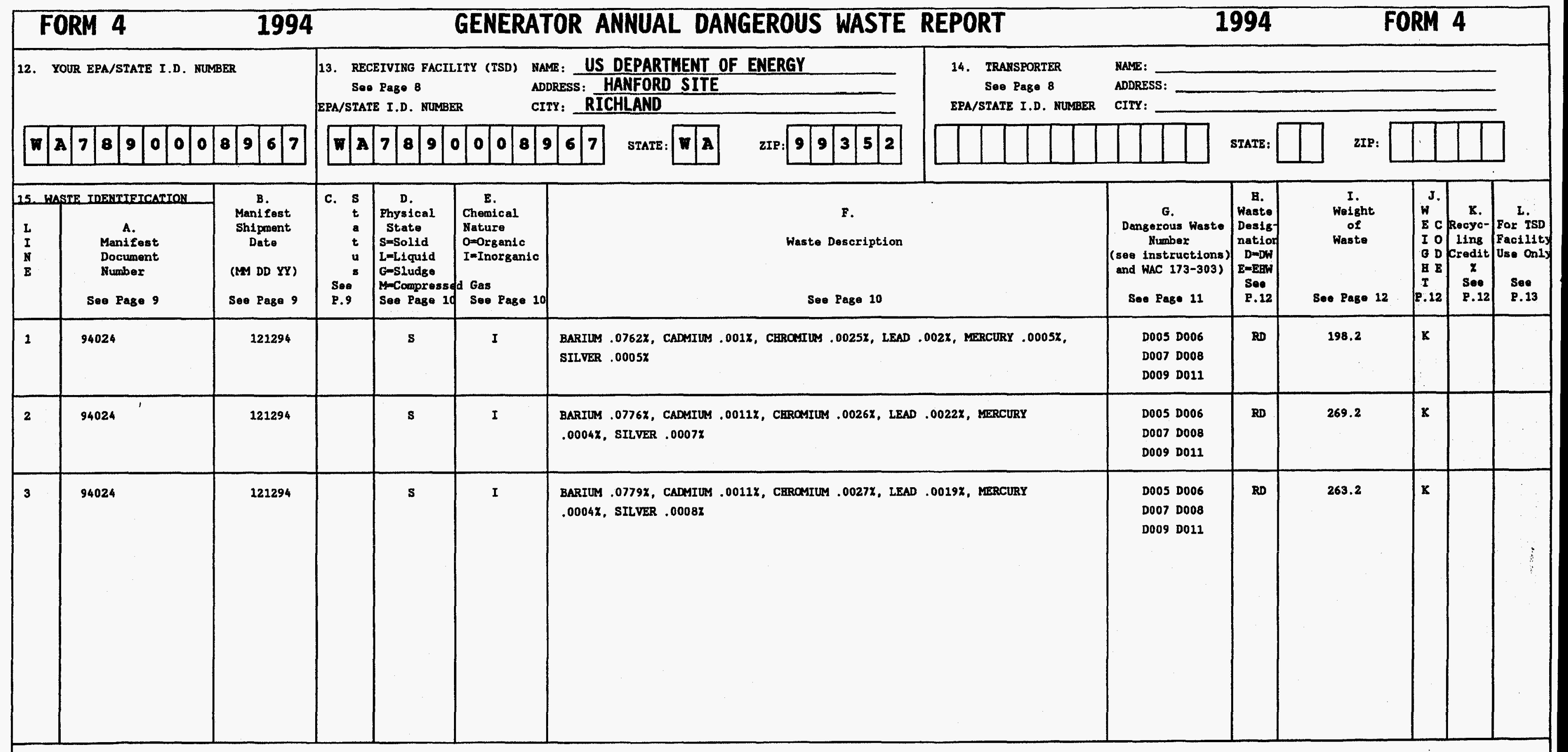

16. COMgENTS (Enter information by section and/or 11ne number--See Page 13). FACILITY: 323 


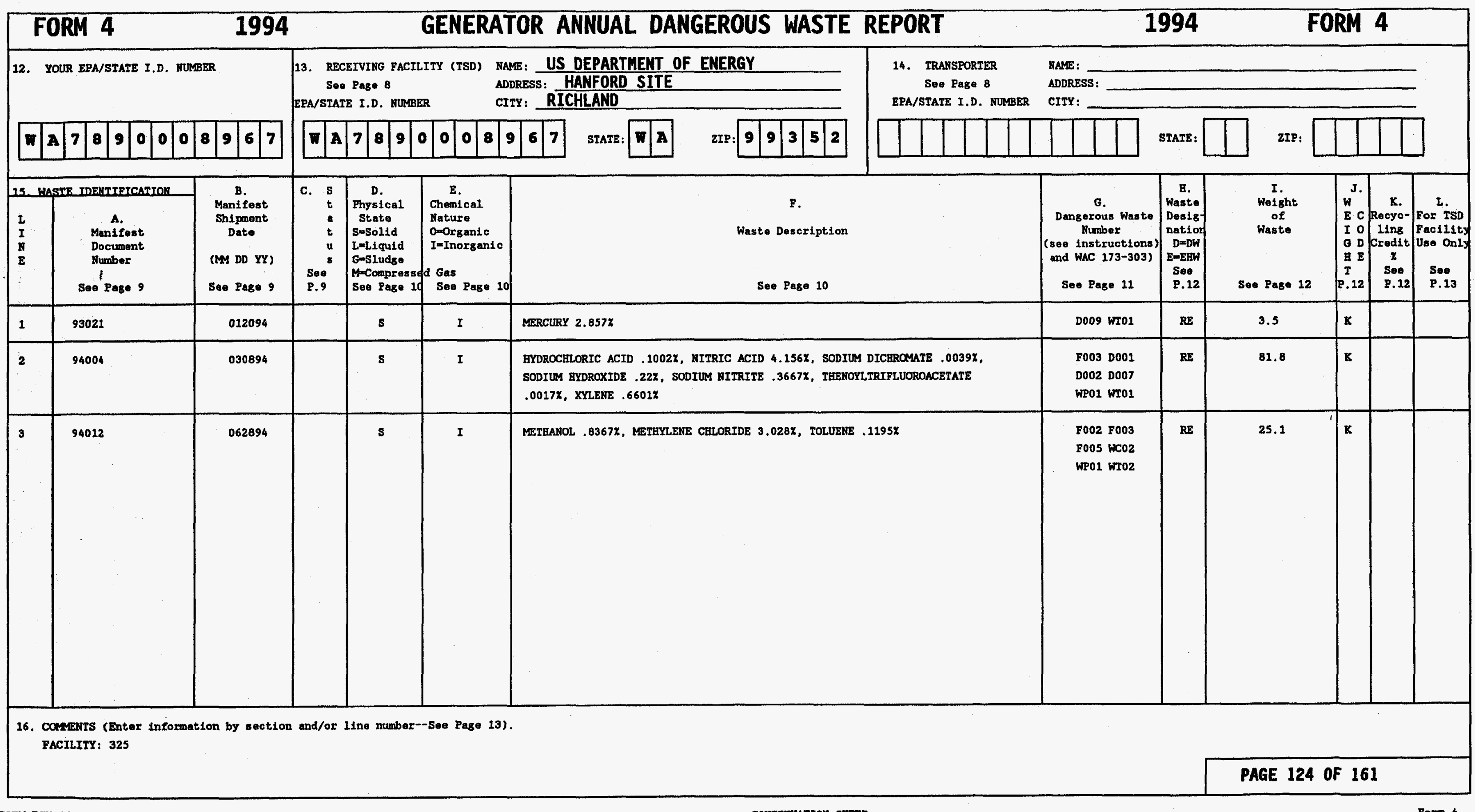




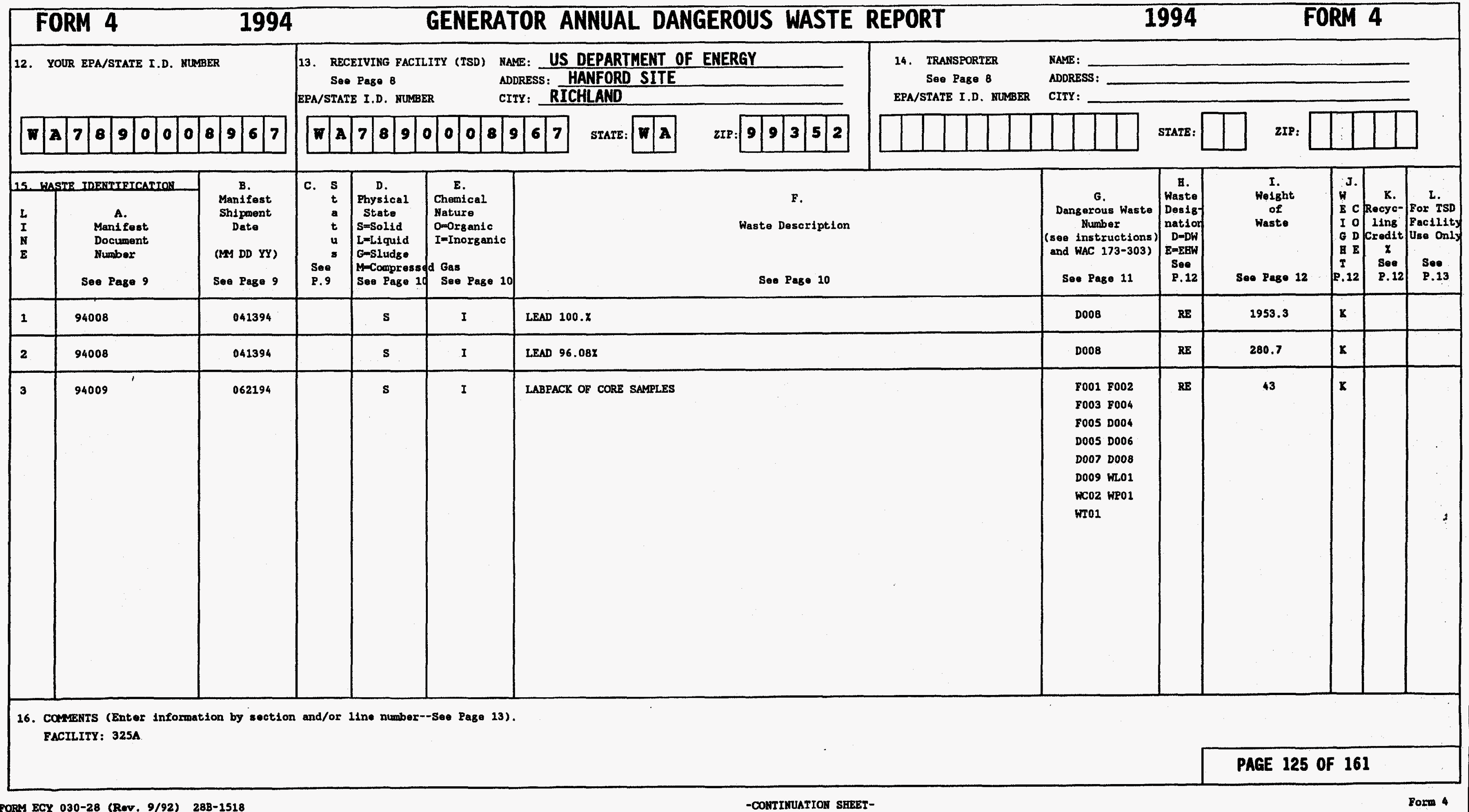




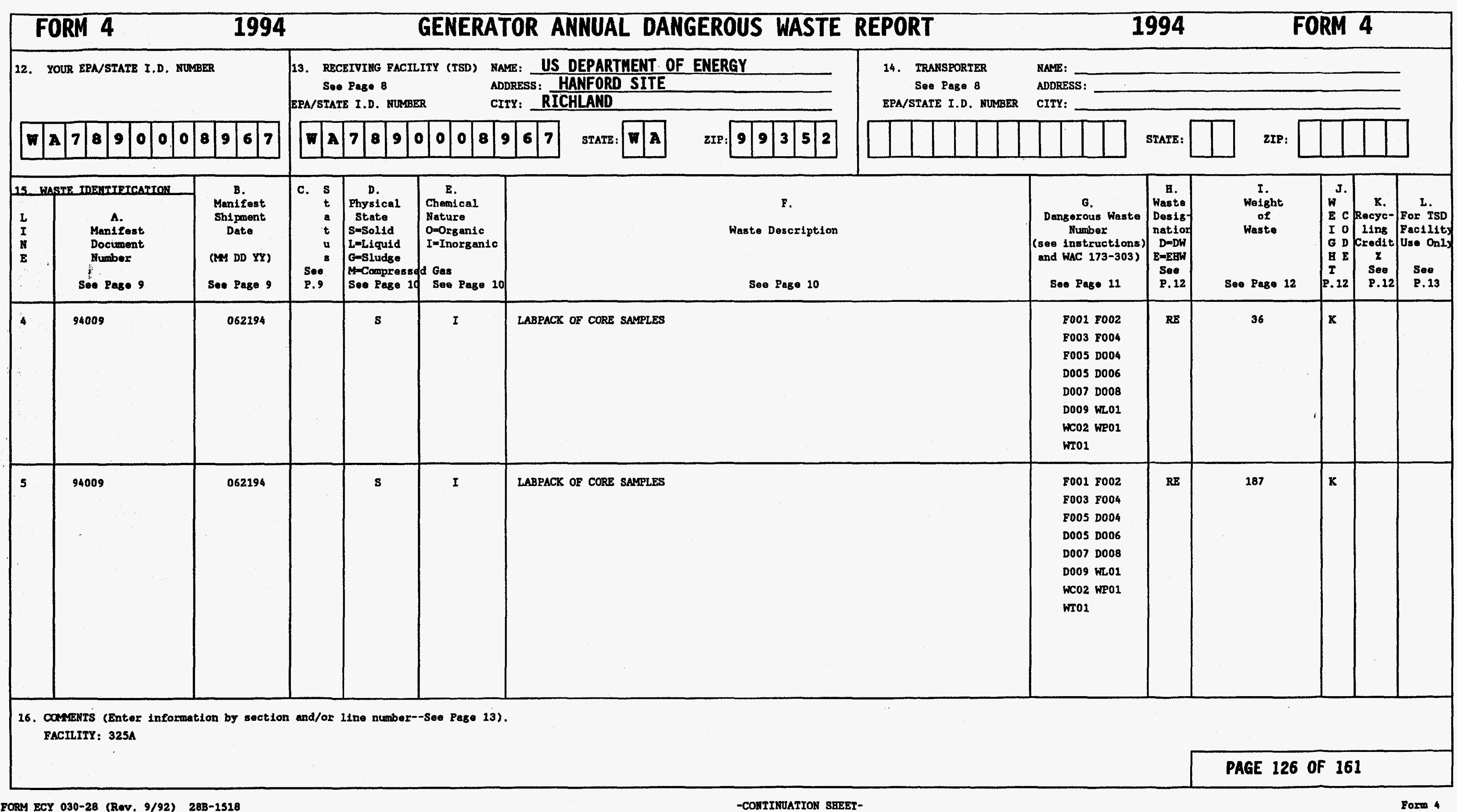




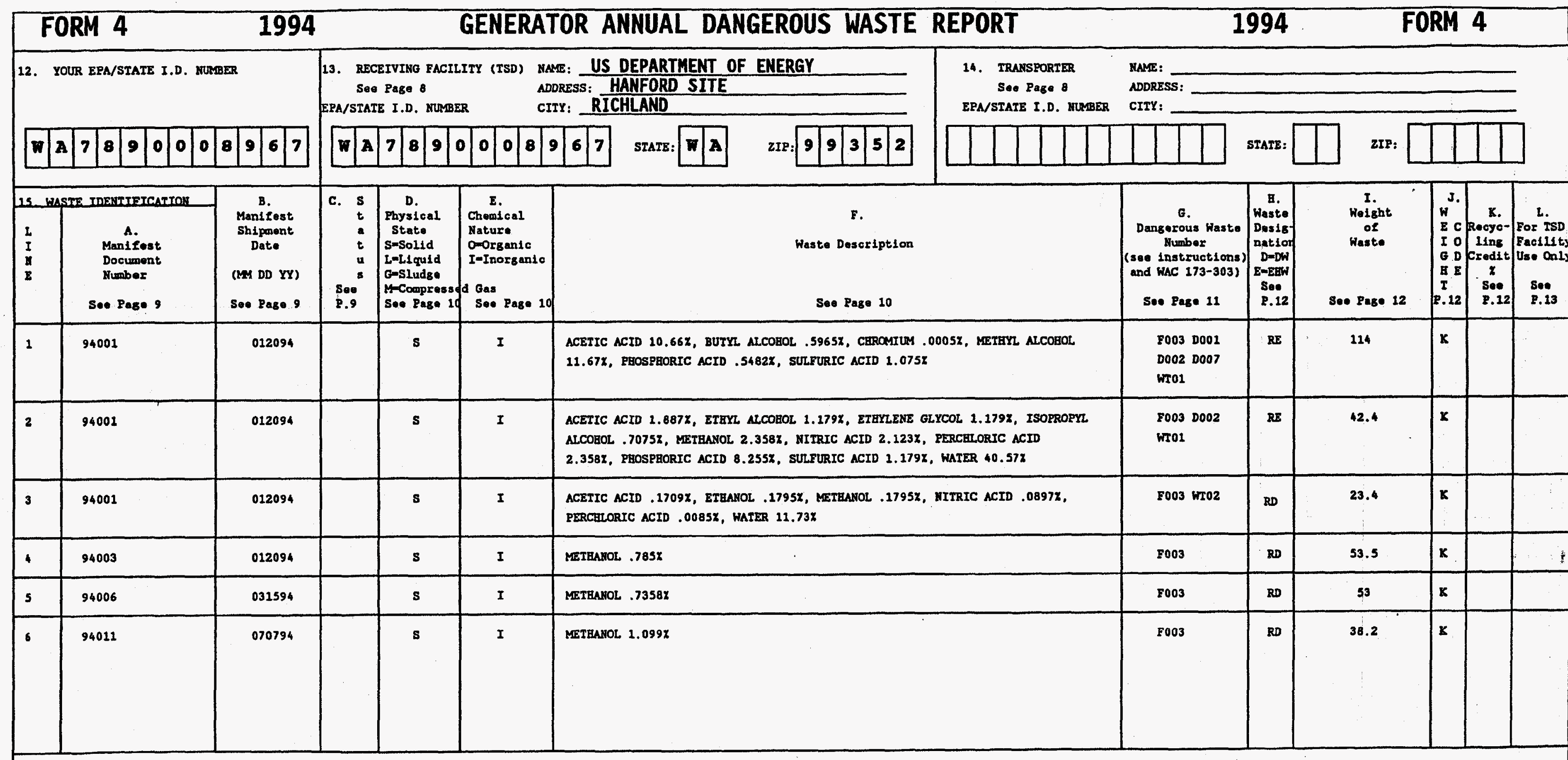

16. Comaris (Enter information by section and/or 11ne number---See Page 13). FACILITY: 326 


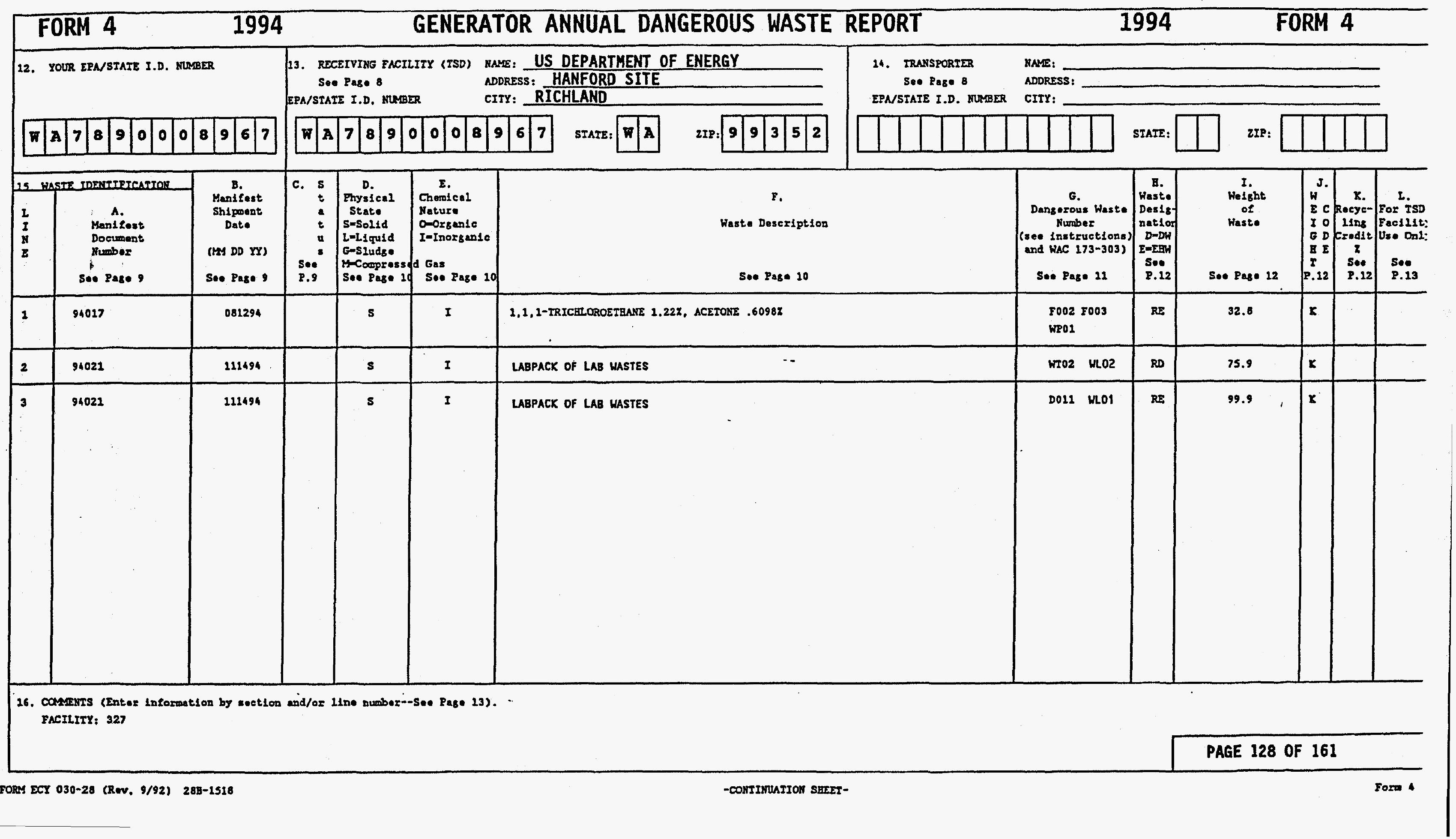




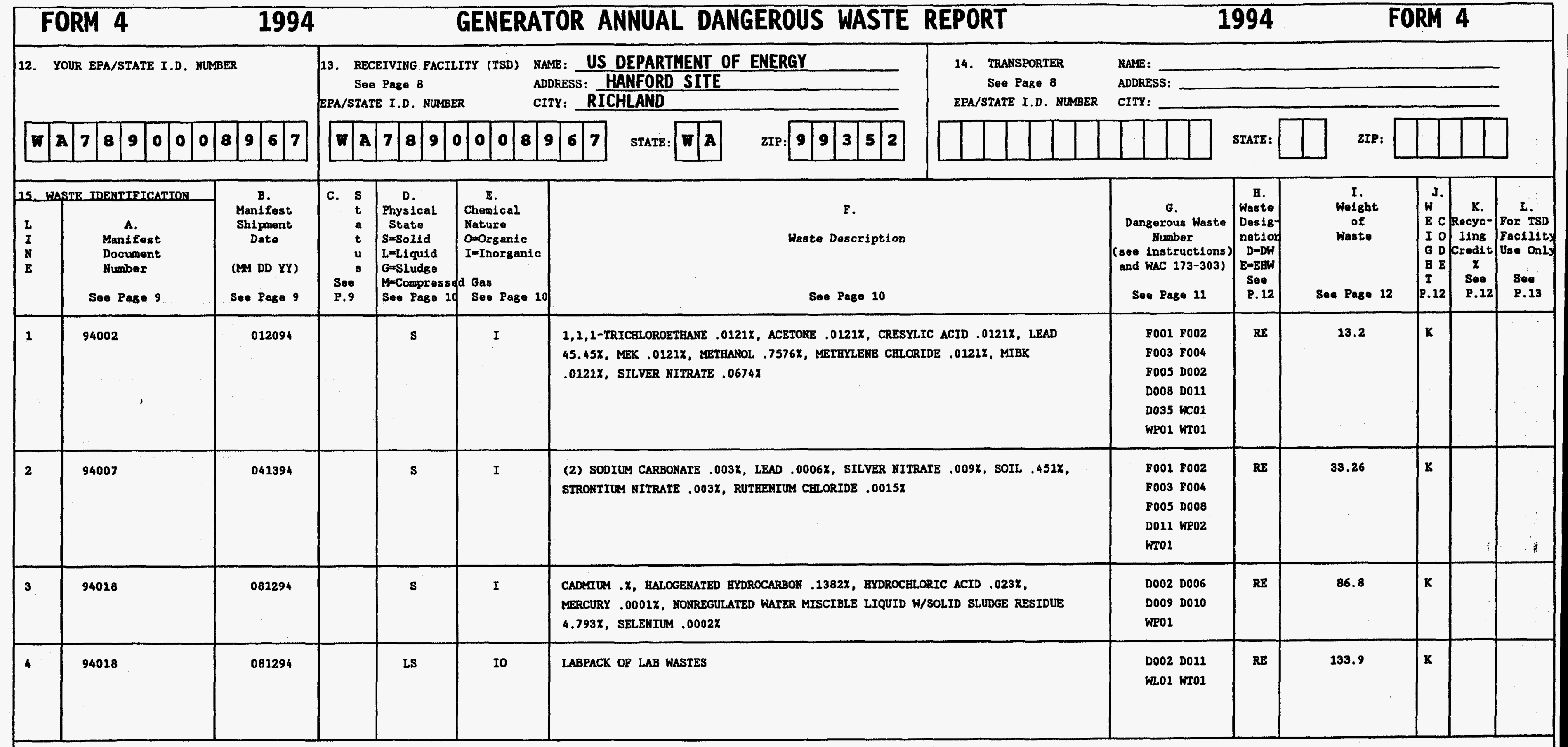

16. Cangerrs (Enter information by section and/or 11ne number--See Page 13). FACILITY: 329

PAGE 129 OF 161 


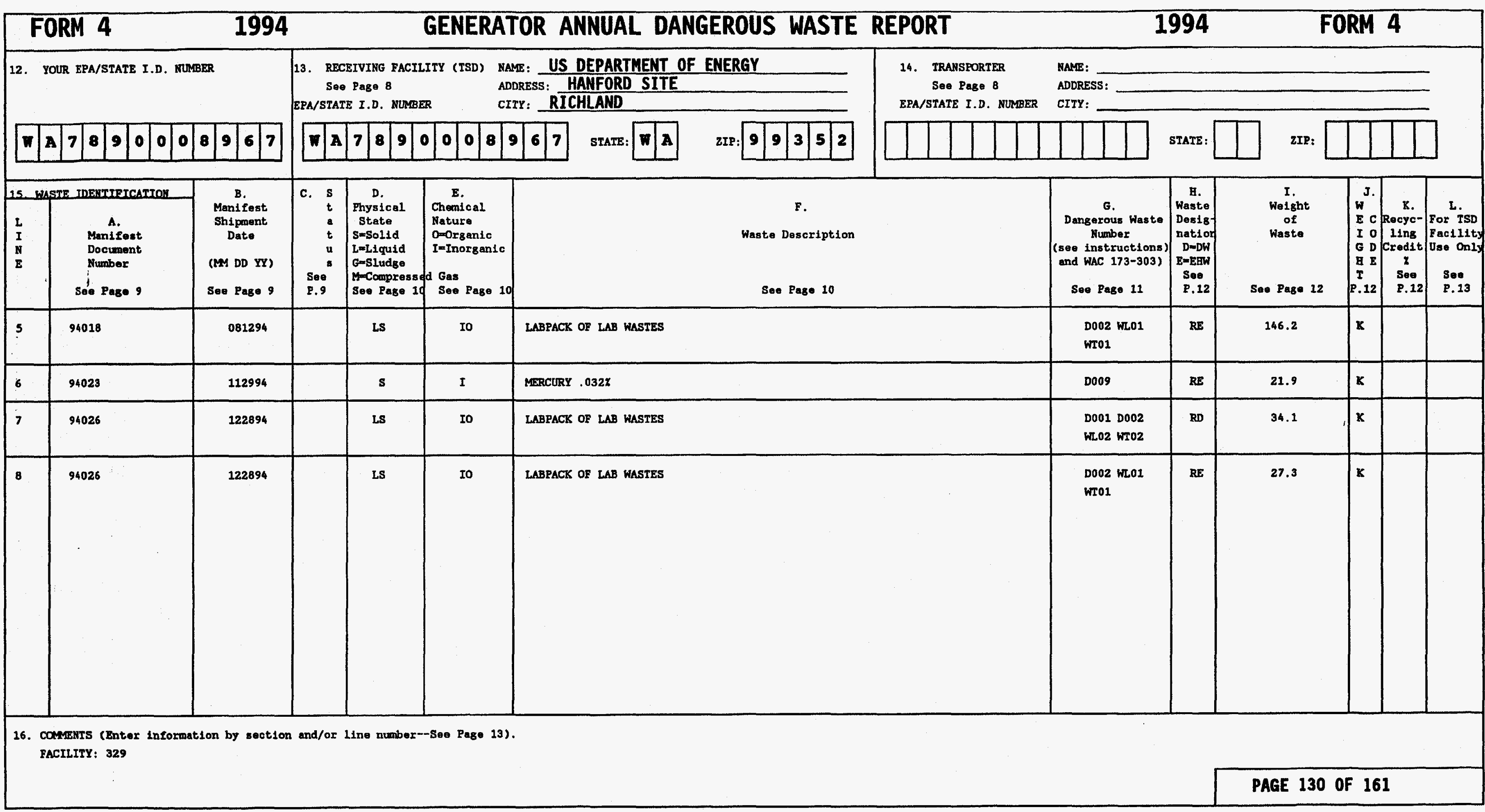




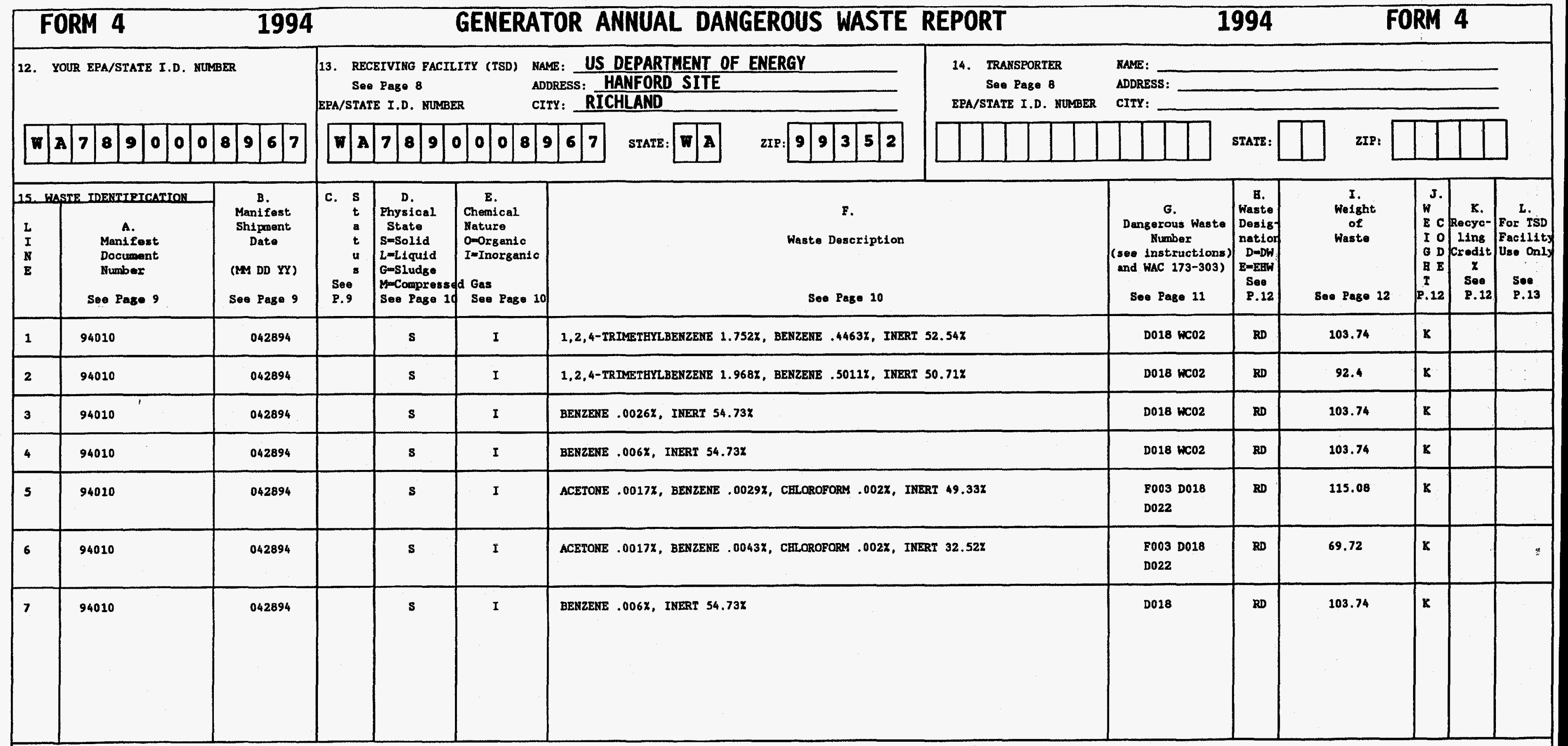

16. CaMENTS (Enter Information by section and/or line number--See Page 13). FACILITY: 331 


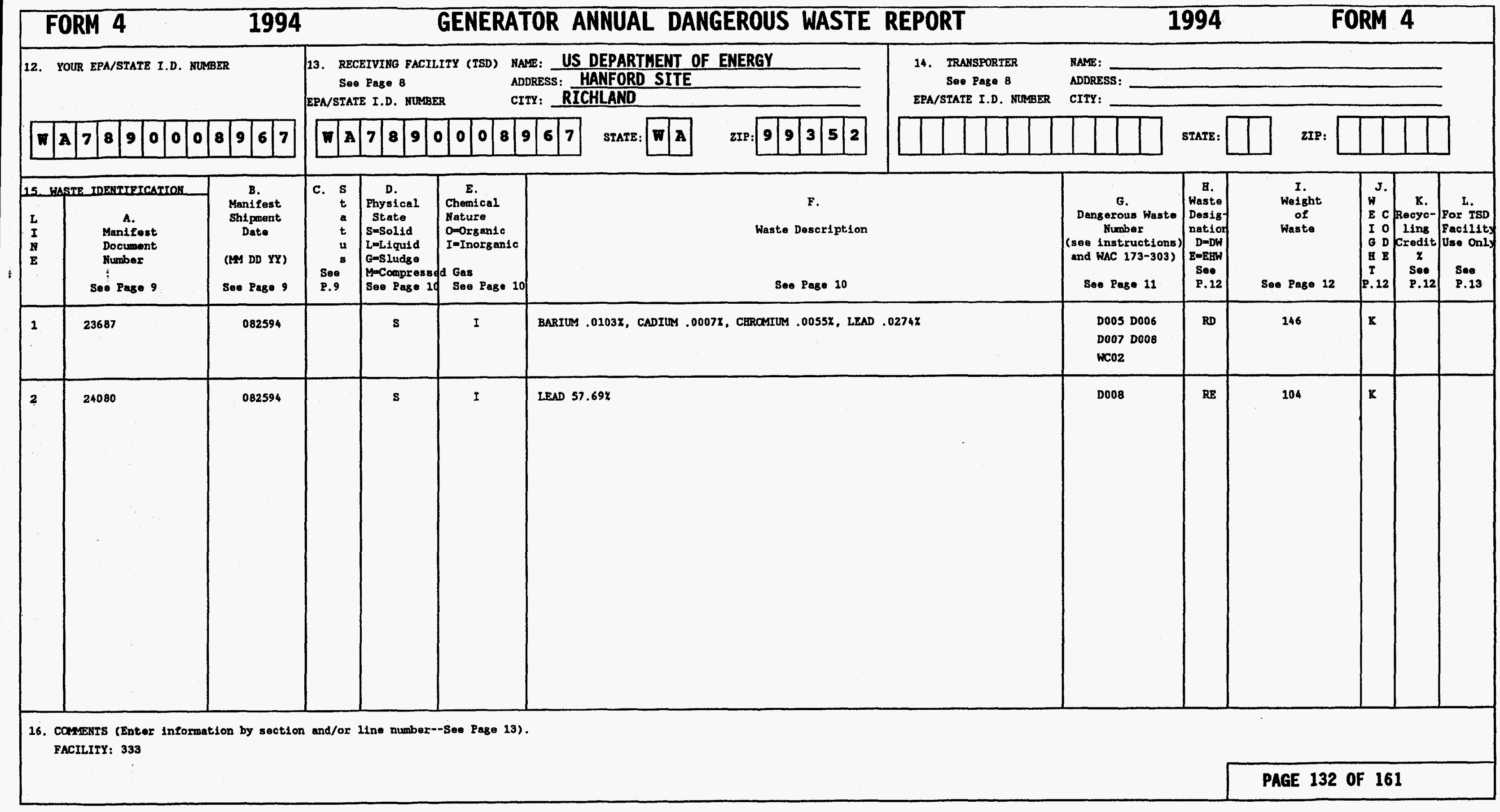




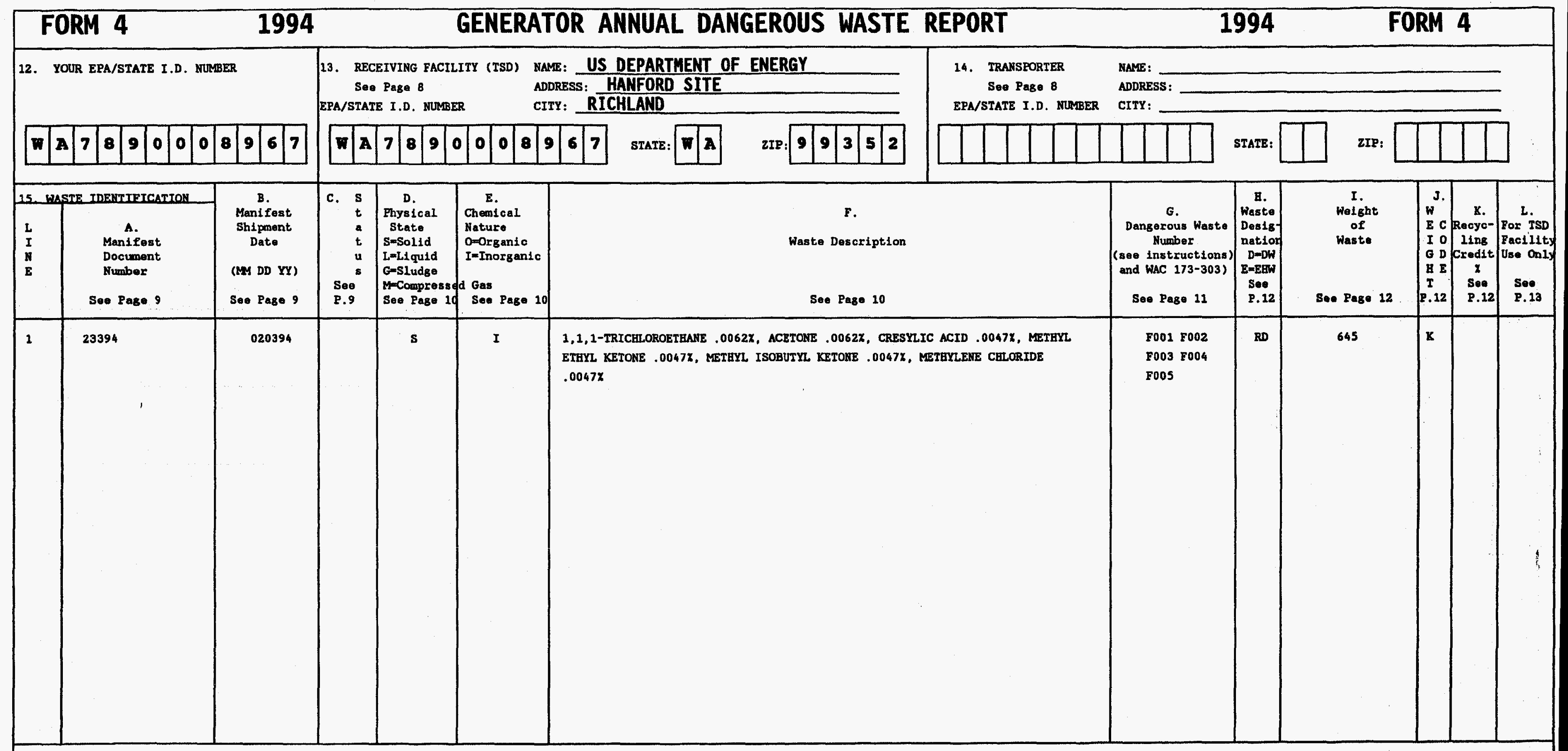

16. comrants (Enter information by section and/or line number--See Page 13). FACILITY: 340

PAGE 133 OF 161 


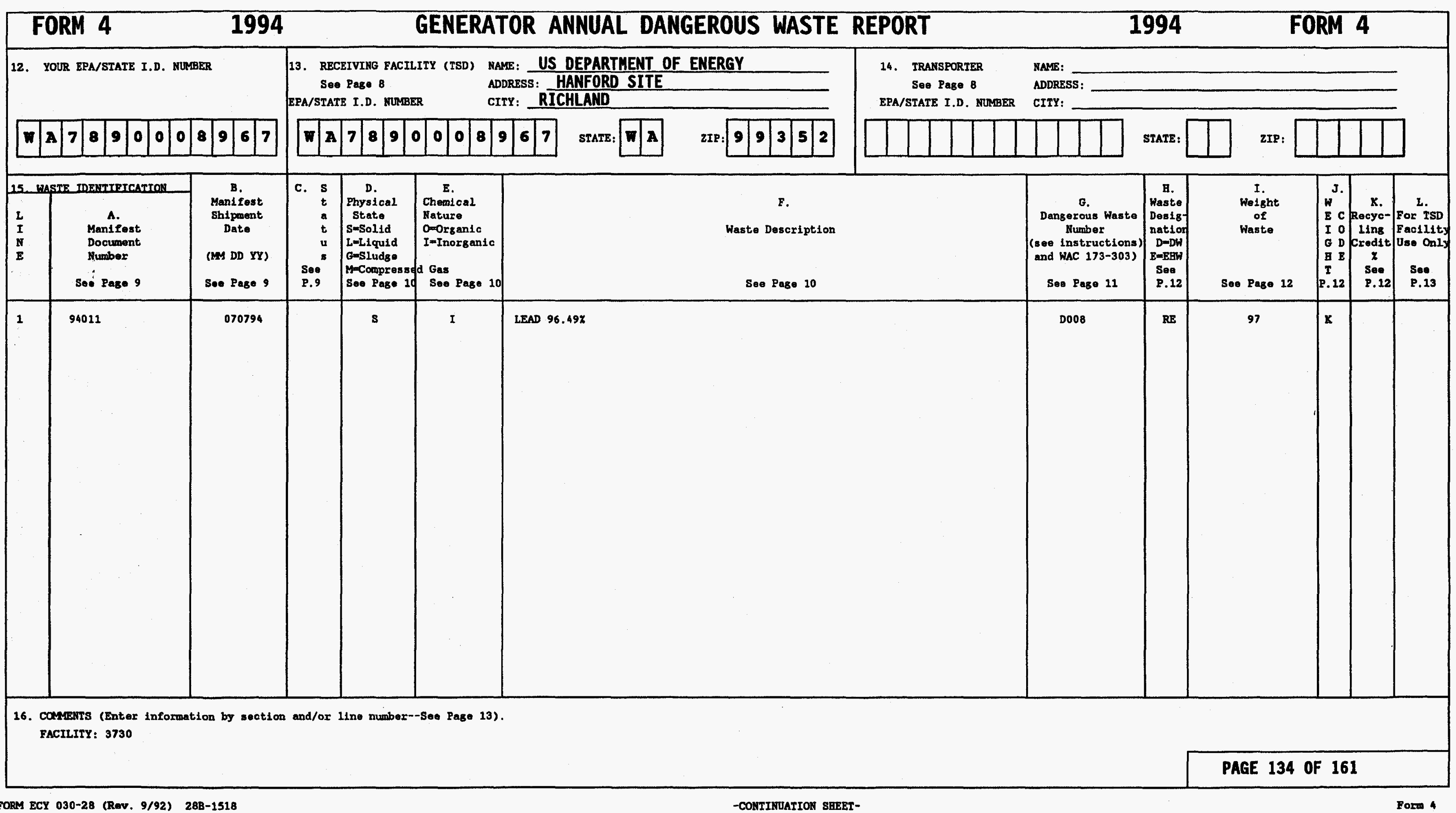




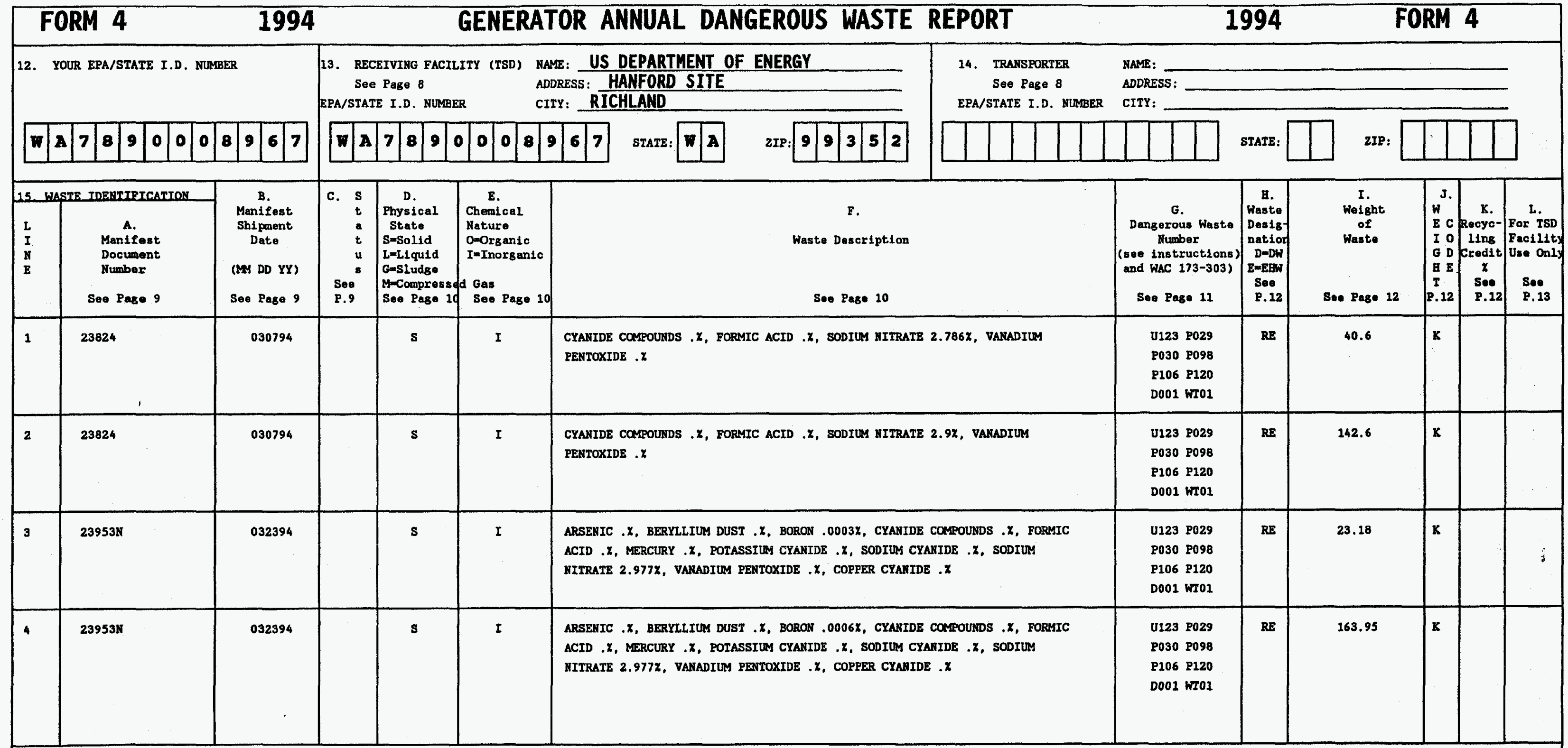

16. CoNMENTS (Enter information by section and/or line number--See Page 13 ). FACILITY: 377 


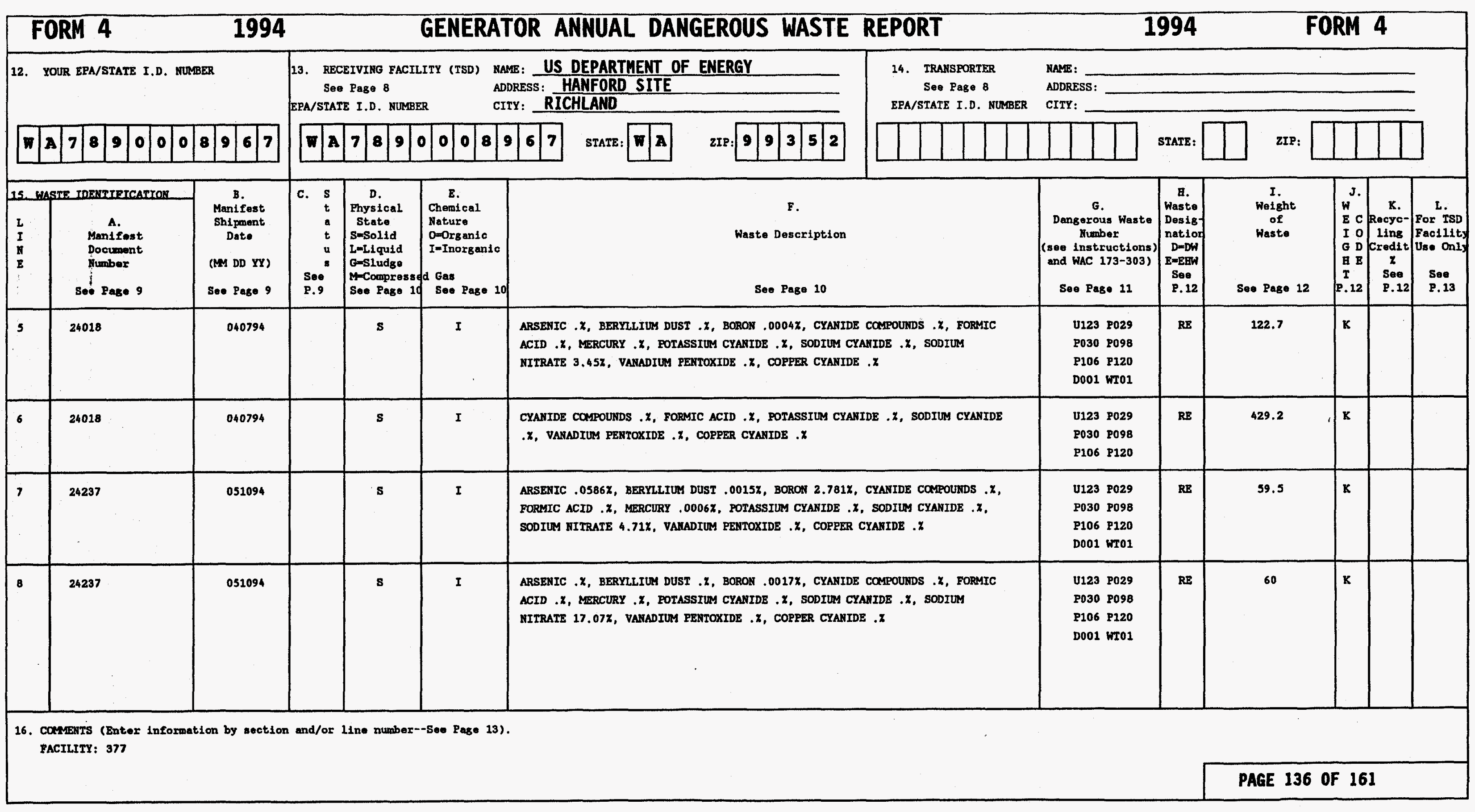




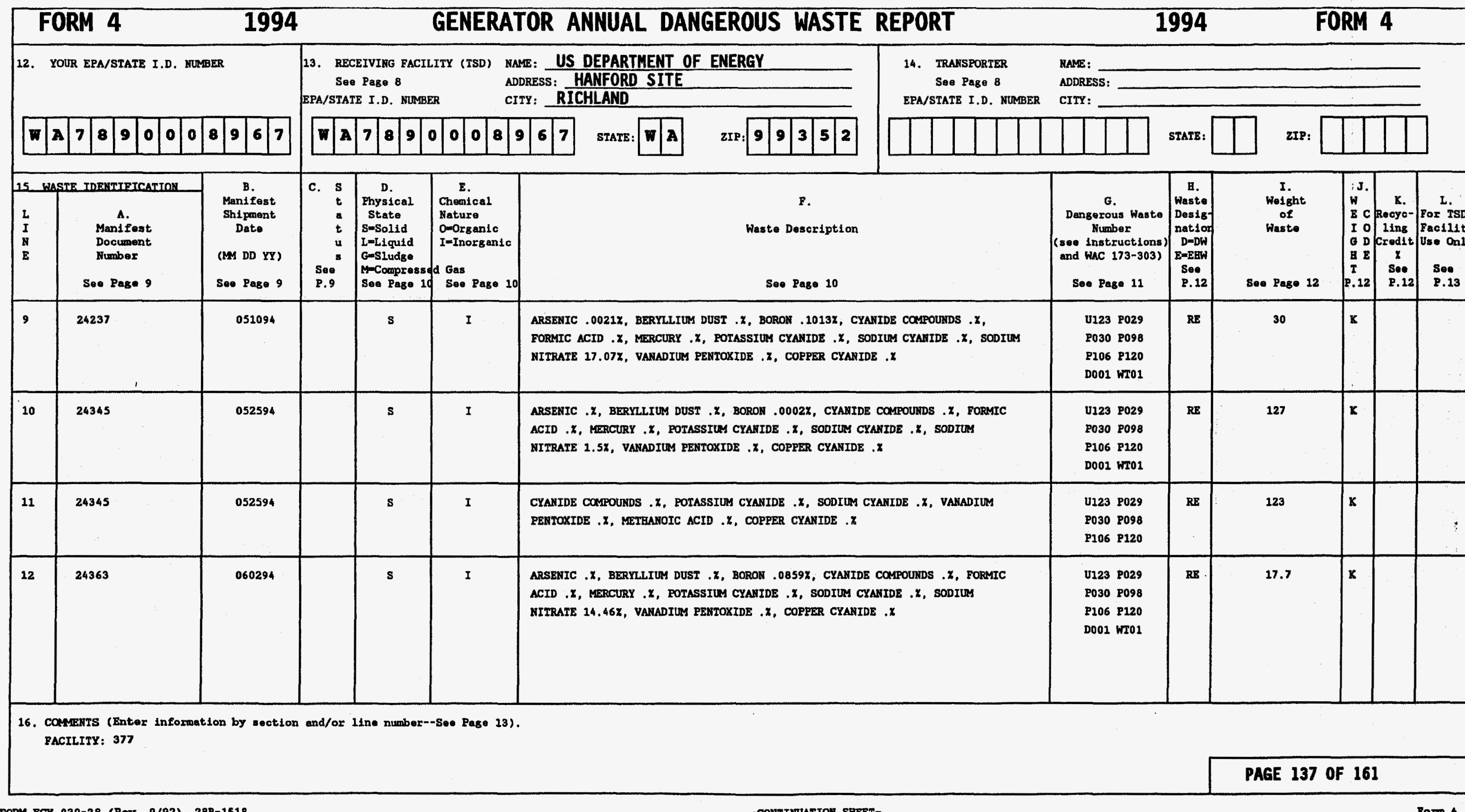




\begin{tabular}{|c|c|c|c|c|c|c|c|c|c|c|c|c|c|}
\hline \multicolumn{2}{|c|}{ FORM 4} & \multirow[t]{2}{*}{1994} & \multicolumn{5}{|c|}{ GENERATOR ANNUAL DANGEROUS WASTE REPORT } & \multicolumn{2}{|c|}{1994} & \multicolumn{3}{|c|}{ FORM 4} & \\
\hline \multicolumn{2}{|c|}{ 12. YOUR EPA/STATE I.D. MUMBERR } & & \multicolumn{4}{|c|}{ 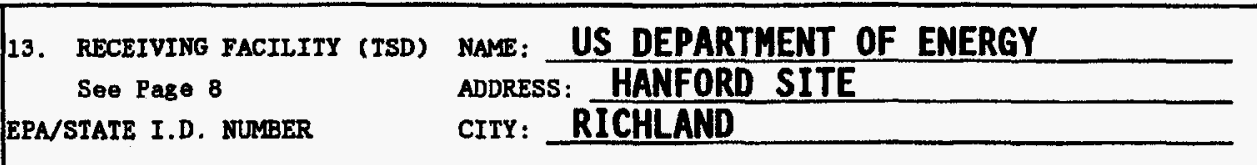 } & $\begin{array}{l}\text { 14. TRANSPORTER } \\
\text { See Page } 8 \\
\text { ERA/STATE I.D. NUMBER }\end{array}$ & \multicolumn{5}{|c|}{$\begin{array}{l}\text { NAME: } \overline{ } \\
\text { ADDRESS: } \square \\
\text { CITY: }\end{array}$} & \\
\hline w & \begin{tabular}{|l|l|l|l|l|l|}
7 & 8 & 9 & 0 & 0 & 1 \\
\end{tabular} & \begin{tabular}{|l|l|l|l|}
8 & 9 & 6 & 7 \\
\end{tabular} & \begin{tabular}{|l|l|}
$\mathbf{T}$ & $\mathbf{A}$ \\
\end{tabular} & \begin{tabular}{|l|l|l|l}
7 & 8 & 9 & 1 \\
\end{tabular} & \begin{tabular}{l|l|l|l|l|}
0 & 0 & 0 & 8 & 9 \\
\end{tabular} & 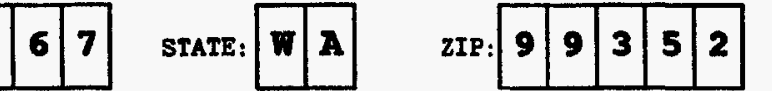 & & & STATE: & zIP: & & & \\
\hline \multicolumn{2}{|c|}{ 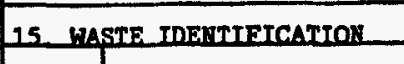 } & \multirow[b]{2}{*}{$\begin{array}{c}\text { B. } \\
\begin{array}{c}\text { Man1fest } \\
\text { Shipwent } \\
\text { Date }\end{array} \\
\text { (MM DD YY) } \\
\text { Soo Page } 9\end{array}$} & \multirow{2}{*}{ 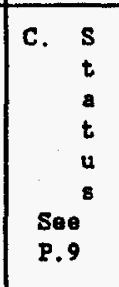 } & \multirow{2}{*}{\begin{tabular}{|l|} 
D. \\
Physical \\
State \\
S=Solid \\
L=Liquid \\
G=sludge \\
M-Compresse \\
Seo Page id \\
\end{tabular}} & \multirow{2}{*}{\begin{tabular}{|l|}
\multicolumn{1}{|c|}{ E. } \\
Chemical \\
Nature \\
O=-Organic \\
I=Inorganic \\
d Gas \\
Seo Page 10
\end{tabular}} & \multirow{2}{*}{\multicolumn{2}{|c|}{$\begin{array}{c}\text { F. } \\
\text { Waste Description } \\
\text { See Page } 10 \\
\end{array}$}} & \multirow{2}{*}{\begin{tabular}{|c|} 
G. \\
Dangerous Waste \\
Number \\
(see instructions) \\
and WAC 173-303) \\
See Page 11 \\
\end{tabular}} & \multirow[b]{2}{*}{ 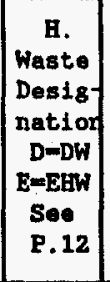 } & \multirow{2}{*}{$\begin{array}{c}\begin{array}{c}\text { I. } \\
\text { Weight } \\
\text { of } \\
\text { Waste }\end{array} \\
\text { See Page } 12\end{array}$} & \multirow{2}{*}{ 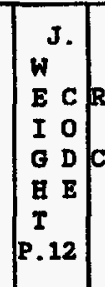 } & \multirow{2}{*}{ 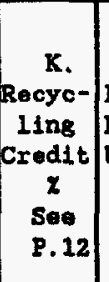 } & \multirow[b]{2}{*}{$\begin{array}{l}\text { L. } \\
\text { For TsD } \\
\text { Factilt } \\
\text { Use onis } \\
\text { Soe. } \\
\text { P.13 }\end{array}$} \\
\hline $\begin{array}{l}2 \\
I \\
\mathbb{R} \\
\mathbf{E}\end{array}$ & $\begin{array}{c}\text { Manifest } \\
\text { Document } \\
\text { Number } \\
\text { S. Page } 9 \\
\text { Seo Pase }\end{array}$ & & & & & & & & & & & & \\
\hline 13 & 24363 & 060294 & & s & I & $\begin{array}{l}\text { ARSENIC } . x \text {, BERYLLIUM DUST } . x \text {, BORON .0968X, CYANTDE } \\
\text { ACID } x \text {, MERCURY } . x \text {, POIASSTIUM CYANIDE } . x \text {, SODTUM CY } \\
\text { NITRAIE } 16.31 x \text {, VANADIUM PENTOXIDE } . x \text {, COPPER CYANID }\end{array}$ & $\begin{array}{l}\text { OMPOURDS } . x, \text { FORMIC } \\
\text { IDE } . x, \text { SODIUM } \\
. x\end{array}$ & $\begin{array}{l}\text { U123 P029 } \\
\text { P030 P098 } \\
\text { P106 P120 } \\
\text { D001 W701 }\end{array}$ & RE & 31.4 & к & & \\
\hline 14 & 24808 & 082694 & & s & I & 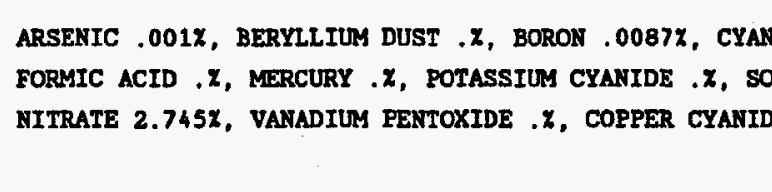 & $\begin{array}{l}\text { E COMPOUNDS } . x \text {, } \\
\text { uM CYANIDE } . x, \text { SODIUM } \\
. x\end{array}$ & $\begin{array}{l}\text { U123 P029 } \\
\text { P030 P098 } \\
\text { P106 P120 } \\
\text { D001 WT01 }\end{array}$ & $\mathrm{RE}$ & 90.1 & $\mathbf{x}$ & & \\
\hline 15 & 25058 & 101294 & & $\mathbf{s}$ & I & 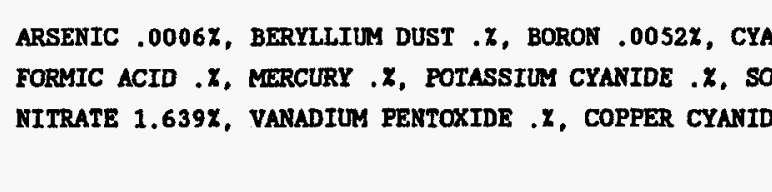 & $\begin{array}{l}\text { DE COMPOUNDS } . x, \\
\text { UM CYANIDE } . x, \text { SODIUM } \\
. x\end{array}$ & $\begin{array}{l}\text { U123 P029 } \\
\text { P030 P098 } \\
\text { P106 P120 } \\
\text { D001 WT01 }\end{array}$ & $\mathrm{RE}$ & 42.7 & $\mathbf{x}$ & & \\
\hline 16 & 25058 & 201294 & & s & I & 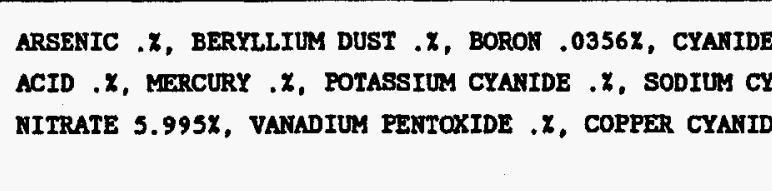 & $\begin{array}{l}\text { OMPOUNDS .x, FORMIC } \\
\text { IDE .x, SODIUM } \\
. x\end{array}$ & $\begin{array}{l}\text { U123 P029 } \\
\text { P030 P298 } \\
\text { P106 P120 } \\
\text { D001 Wro1 }\end{array}$ & $\mathrm{RE}$ & 71.8 & $x$ & & \\
\hline \multicolumn{14}{|c|}{$\begin{array}{l}\text { 16. Comyerrs (Entex information by section and/ox Iine number--See Page, 13). } \\
\text { FACILITY: } 377\end{array}$} \\
\hline & & & & & & & & & & PAGE 13 & 161 & & \\
\hline
\end{tabular}




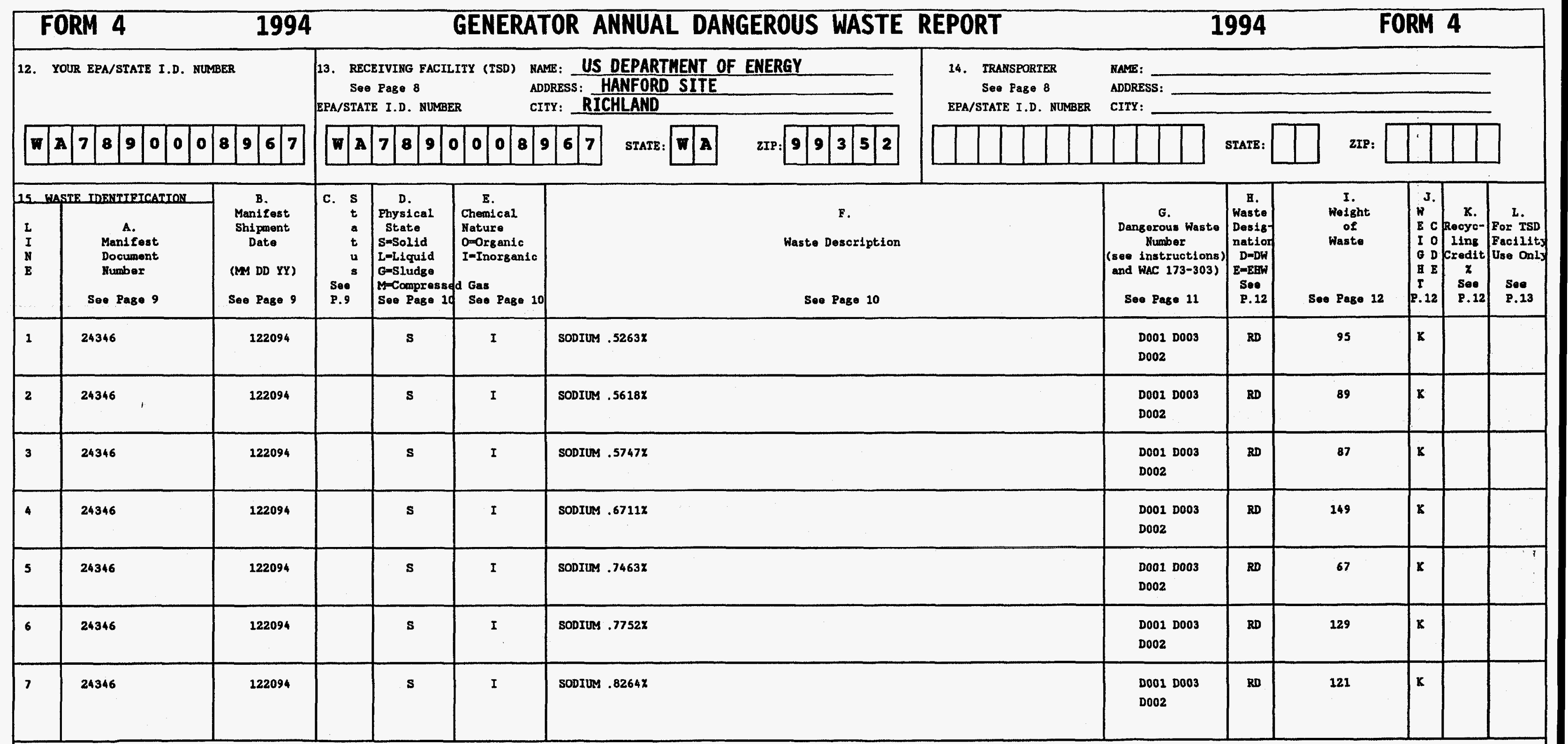

PAGE 139 OF 161

CONENTS (Enter information by section and/or line number--See Page 13). FACILITY: 4843 


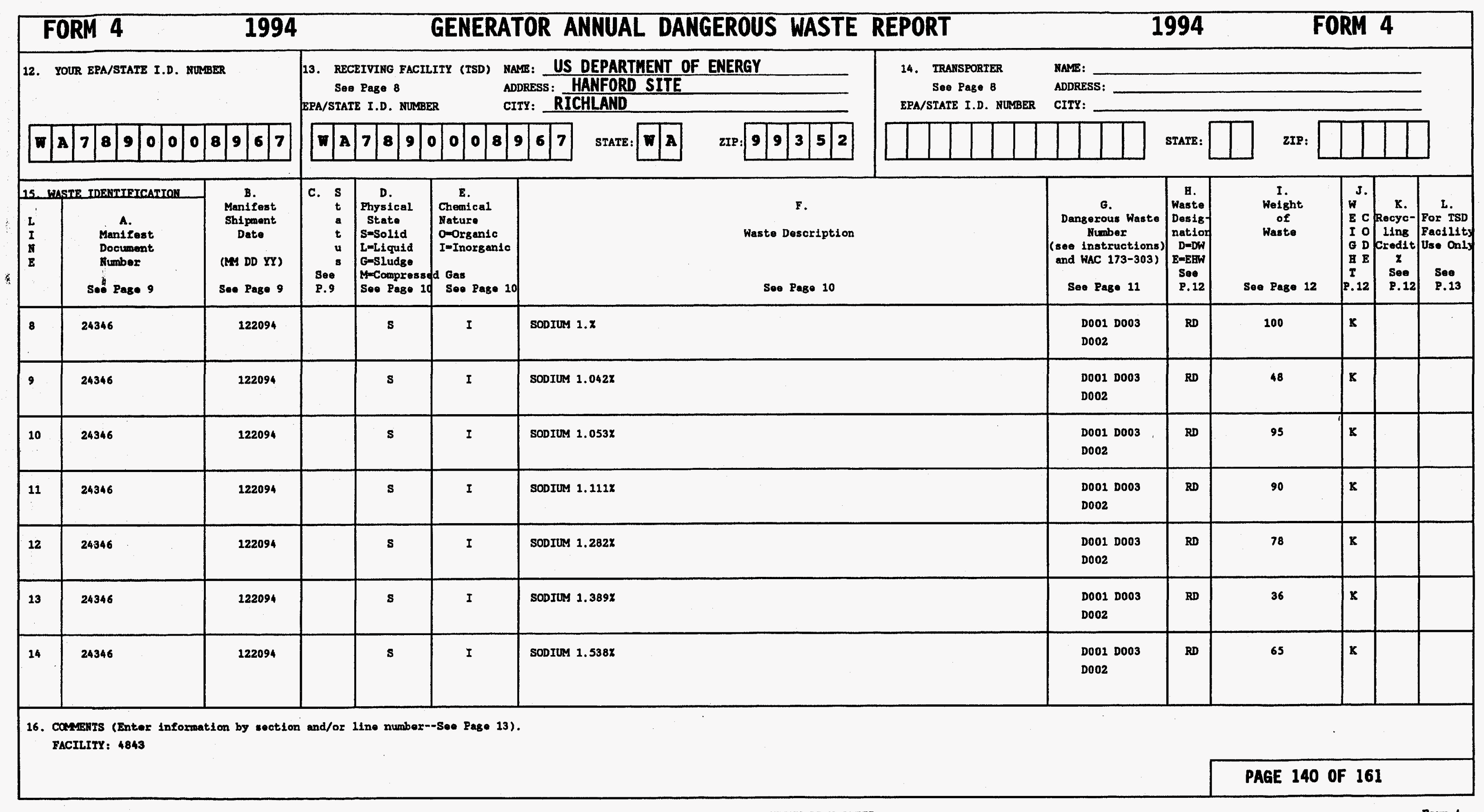




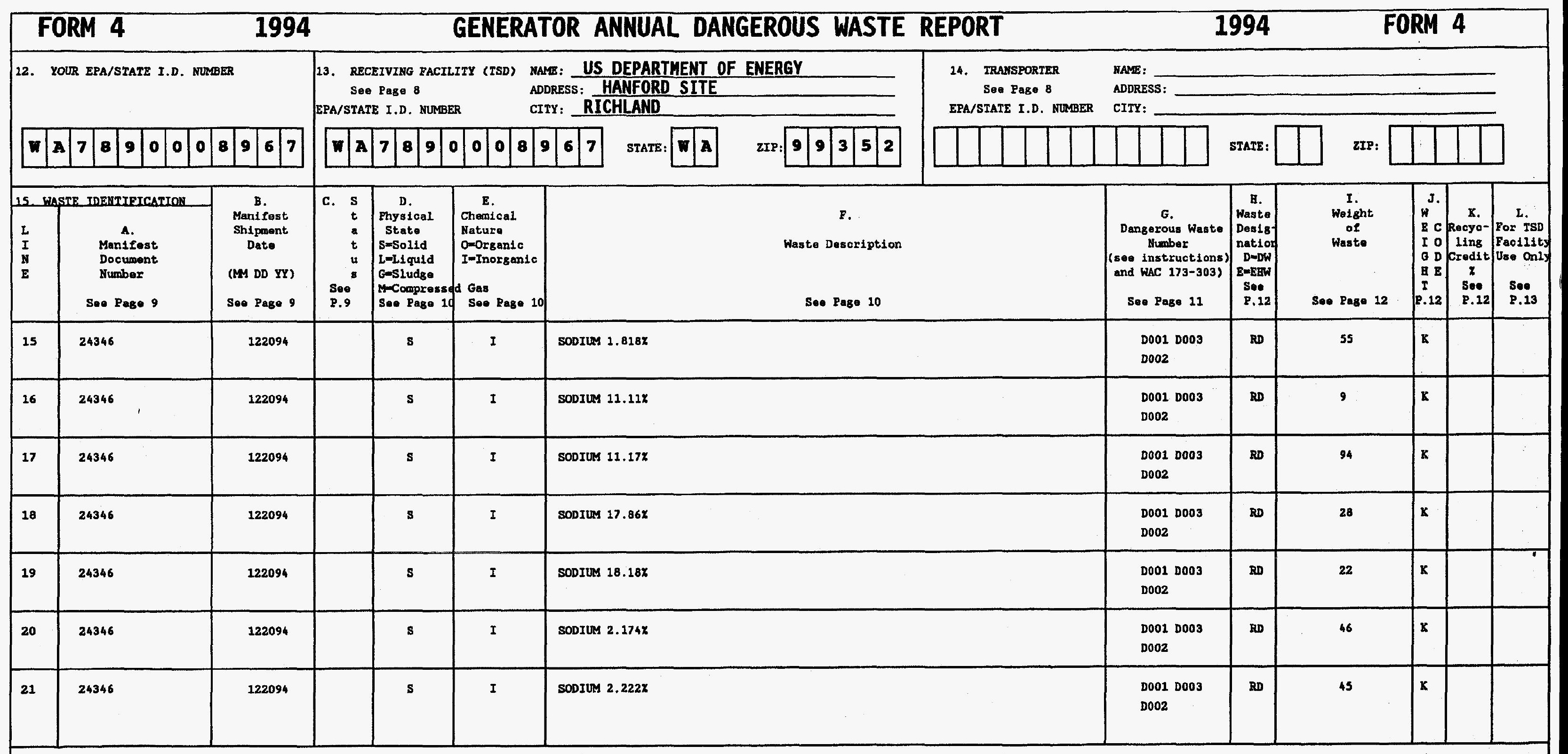

16. COMgeNrs (Entox information by section and/or 11ne number--See Page 13). FACILITY: 4843 


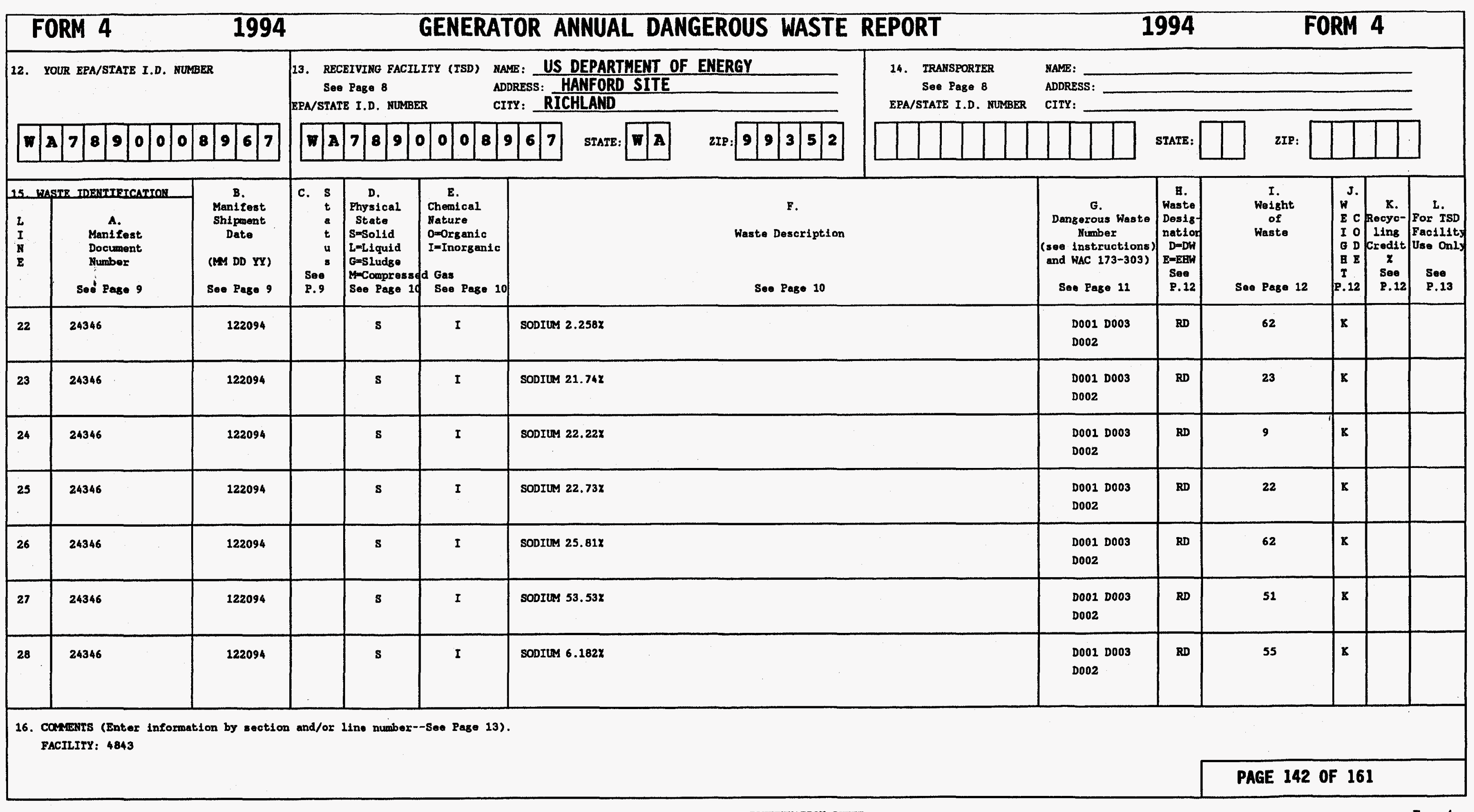




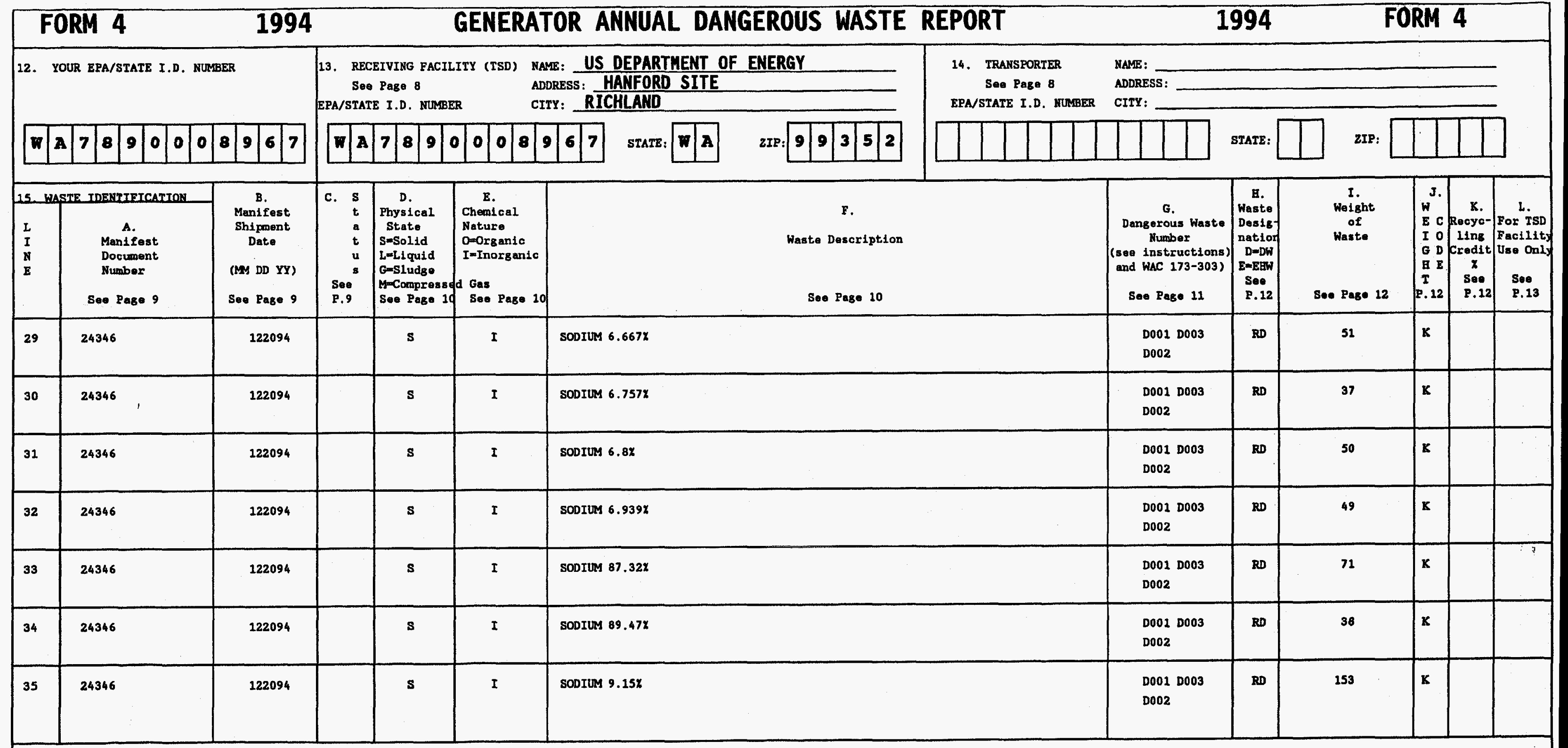

16. CoMmenrs (Enter information by soction and/or 1ine number--See Page 13). FACILITY : 4843 


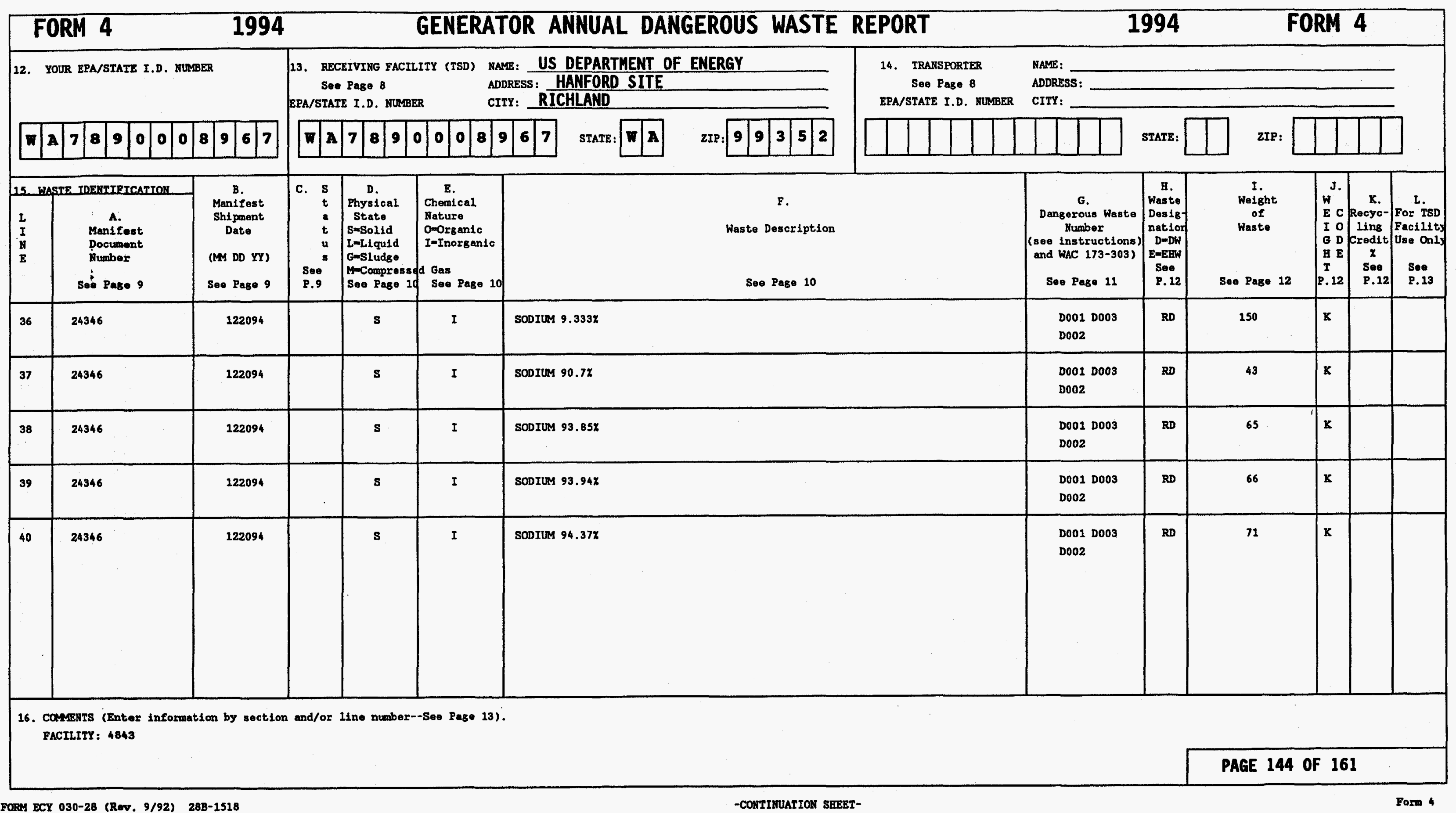




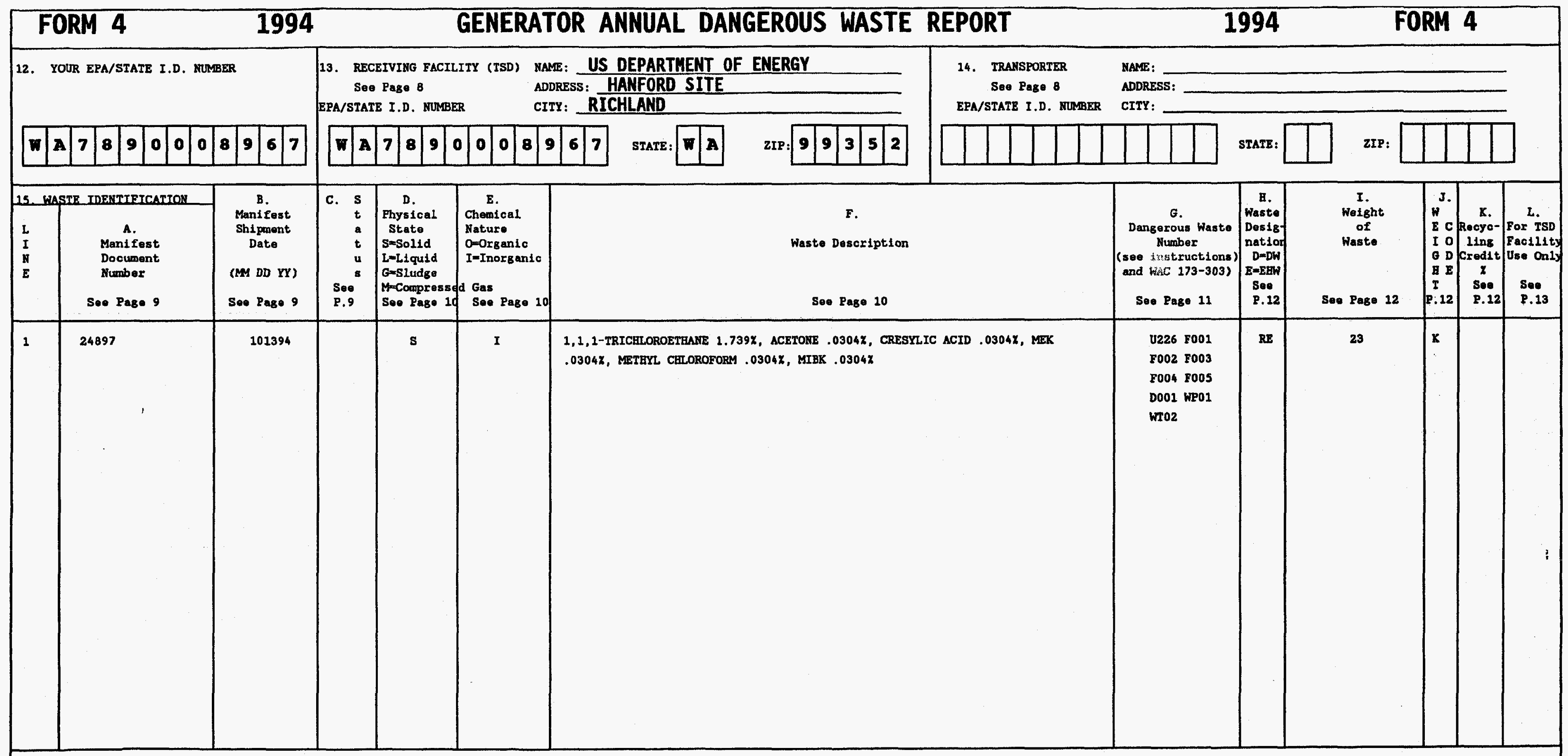

16. comeNIS (Enter information by section and/or line number--See Page 13). FACILITY: CWC

PAGE 145 OF 161 


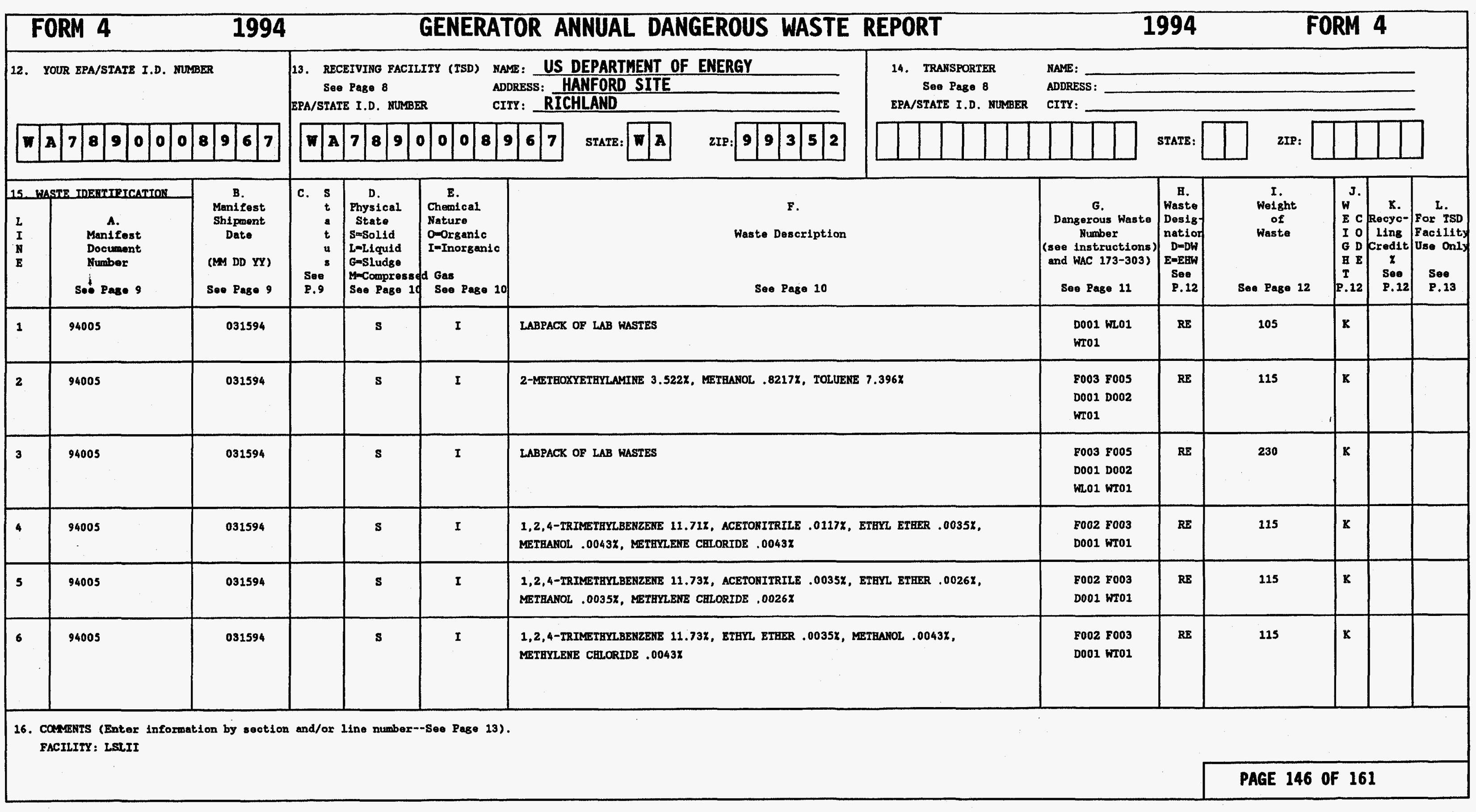




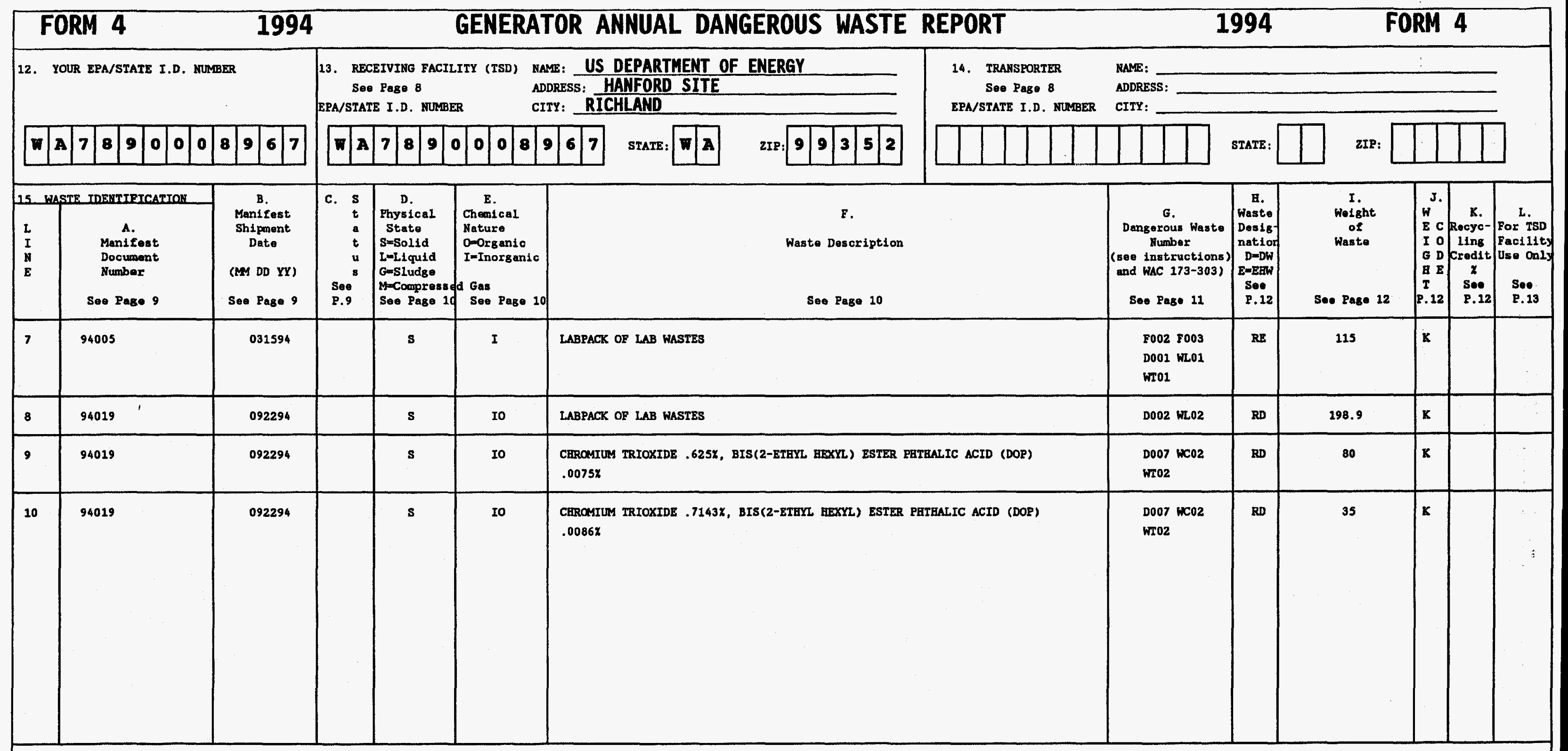

16. coments (Enter information by section and/or 1ine number--Soe Page 13). FACILITY: LSLII 


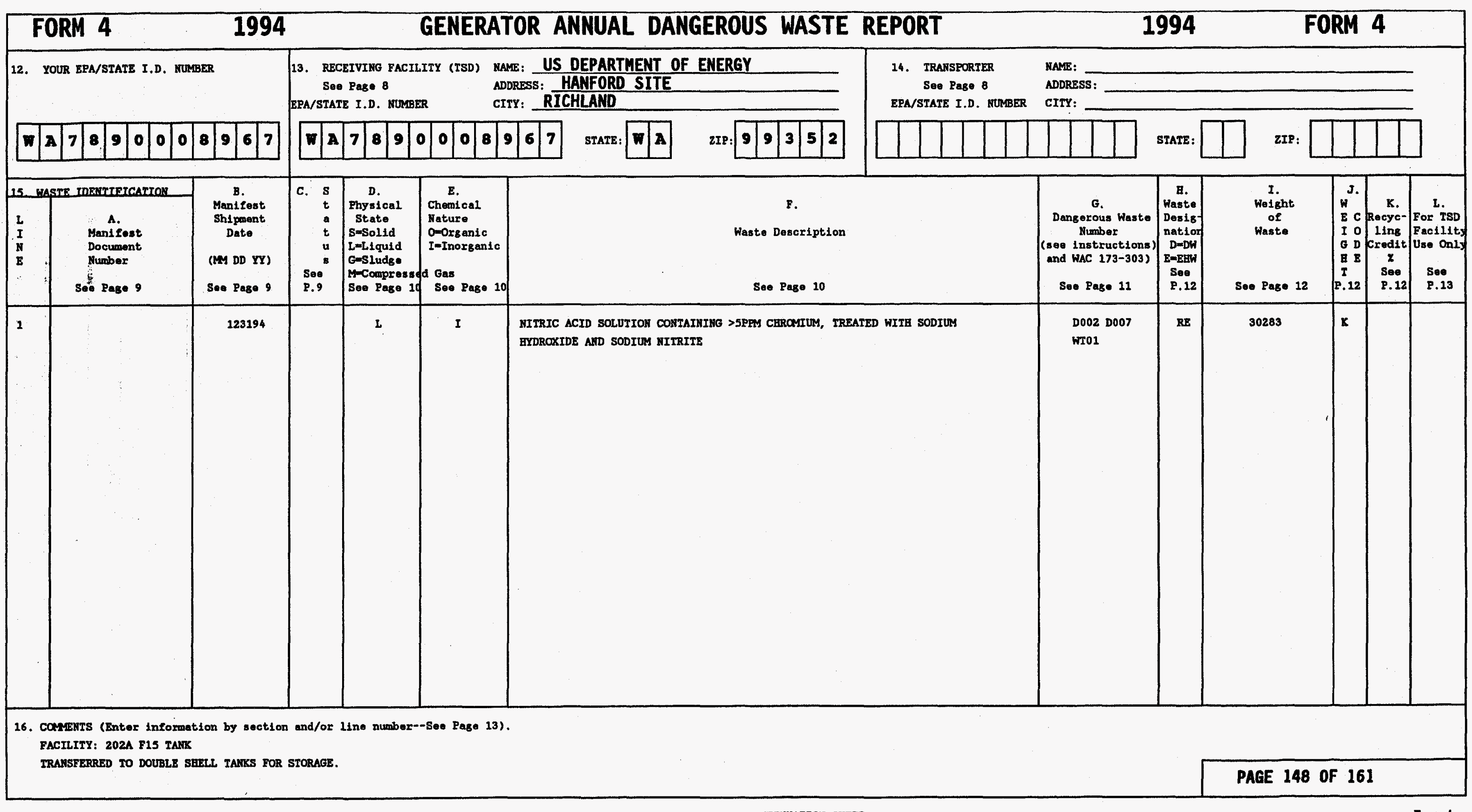




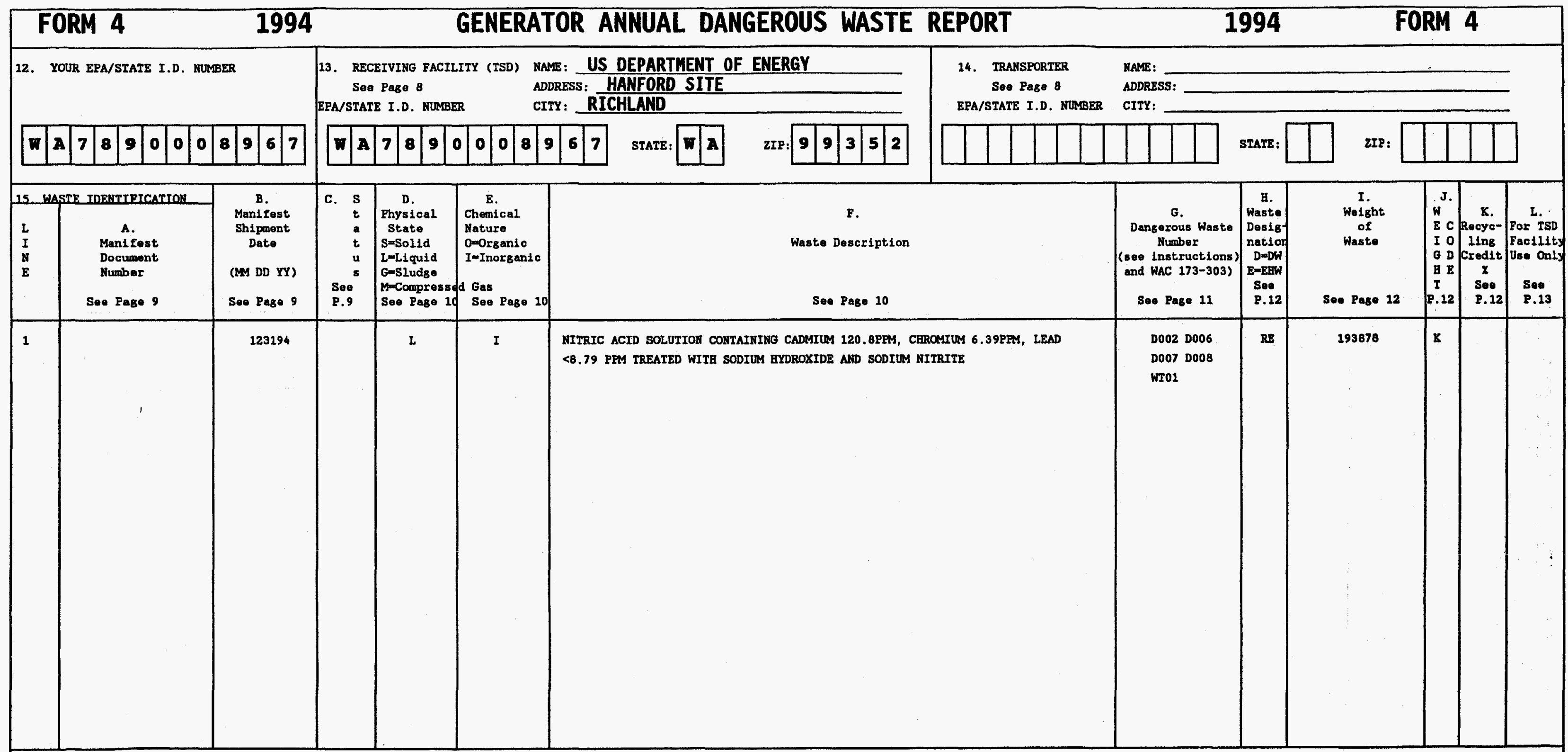

16. covenENTs (Enter information by soction and/or line number--See Page 13), FACILITY: $202 A$ F18 IANKS

TRANSFERRED TO DOUBLE SHELL TANKS FOR STORAOE.

PAGE 149 OF 161 


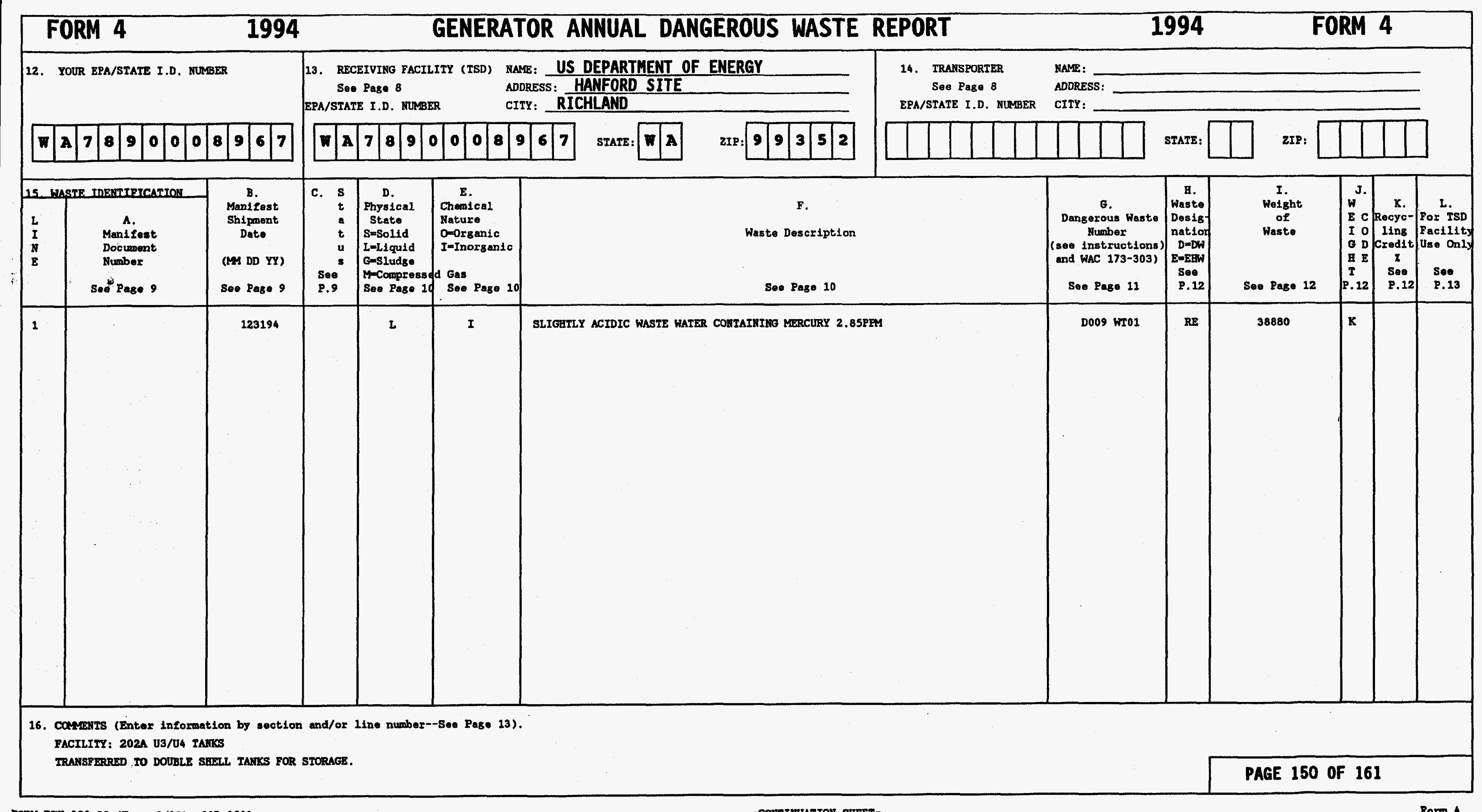




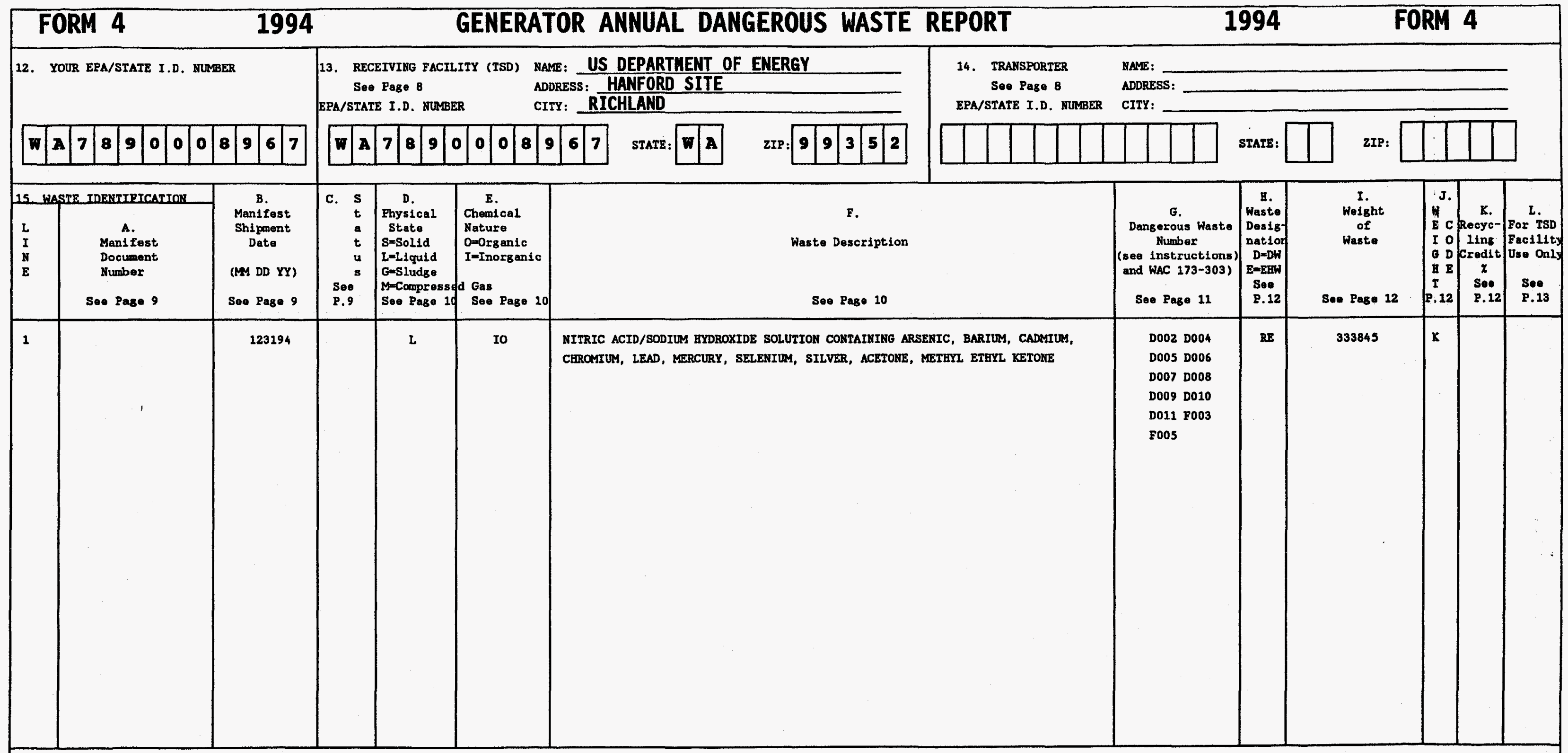

16. COMENTS (Enter information by section and/or line number--Soe Page 13). FACILITY: 204AR URLOADING STA.

TRANSFERRED TO DOUBLE SHELL, TANKS FOR STORAGE.

PAGE 151 OF 161 


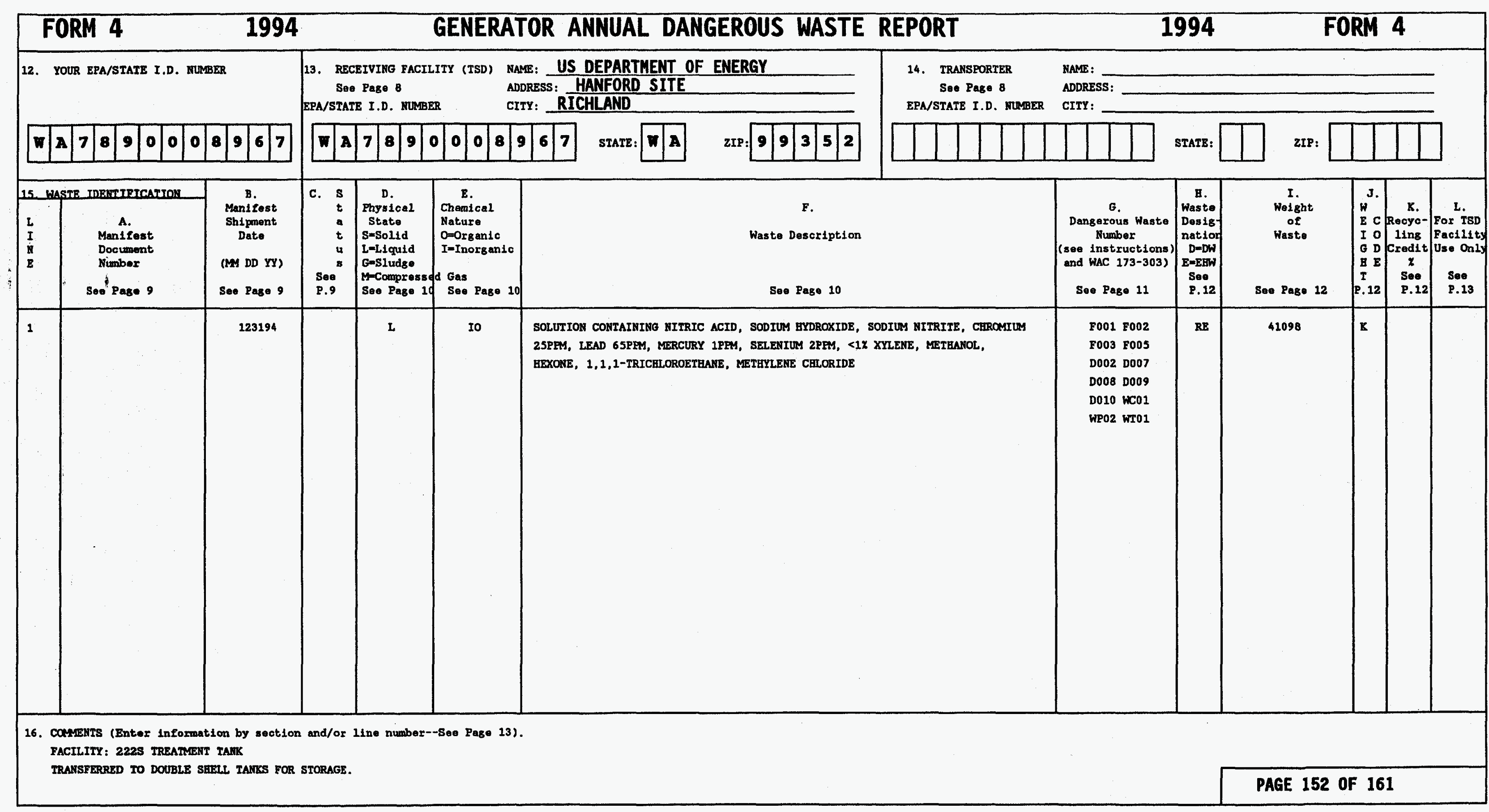




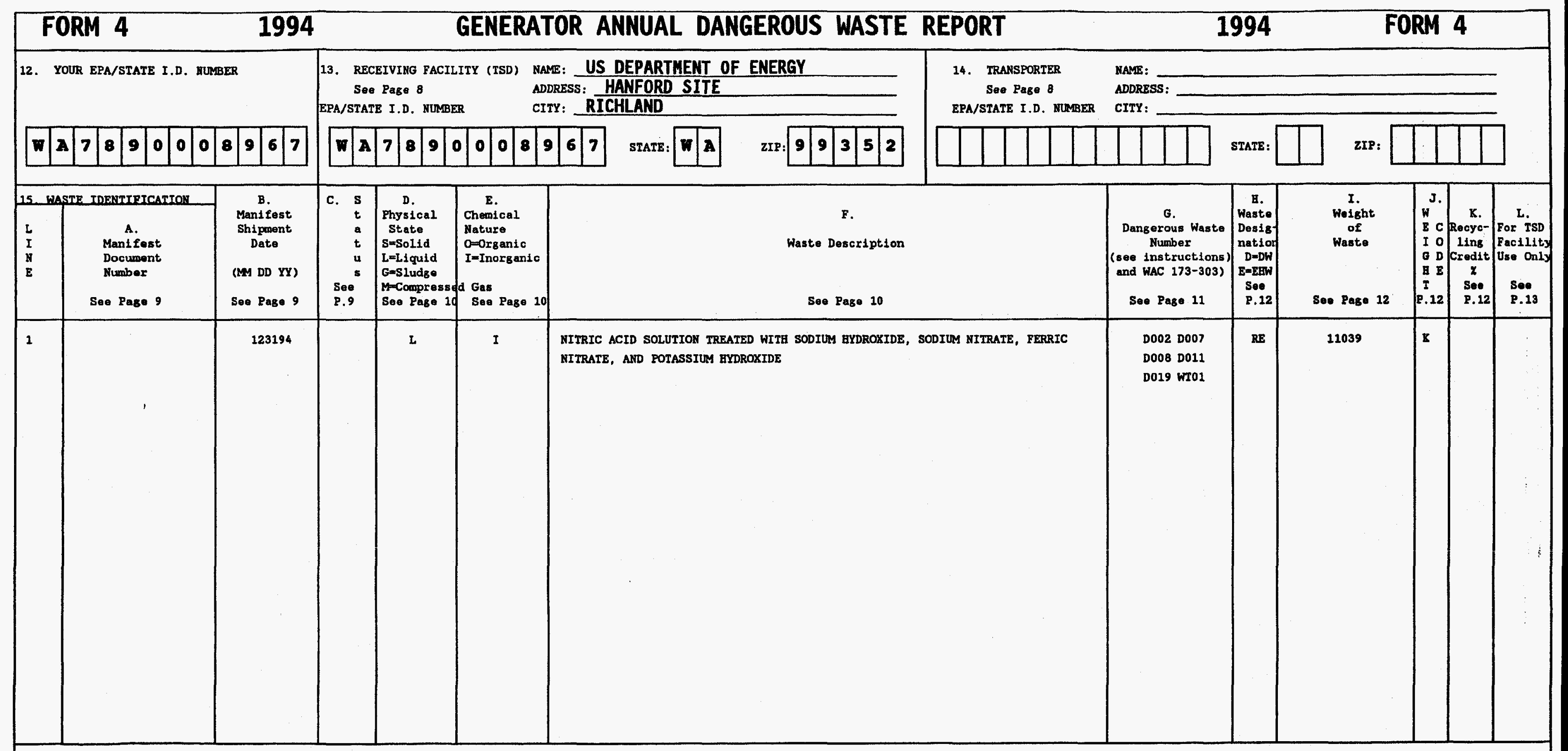

16. Camerrs (Enter information by section and/or line number--See Page 13).

FACILITY: 2412 TREATMENT TANK

TRANSFERRED TO DOUBLE SHELL TANKS FOR STORAGE.

PAGE 153 OF 161 


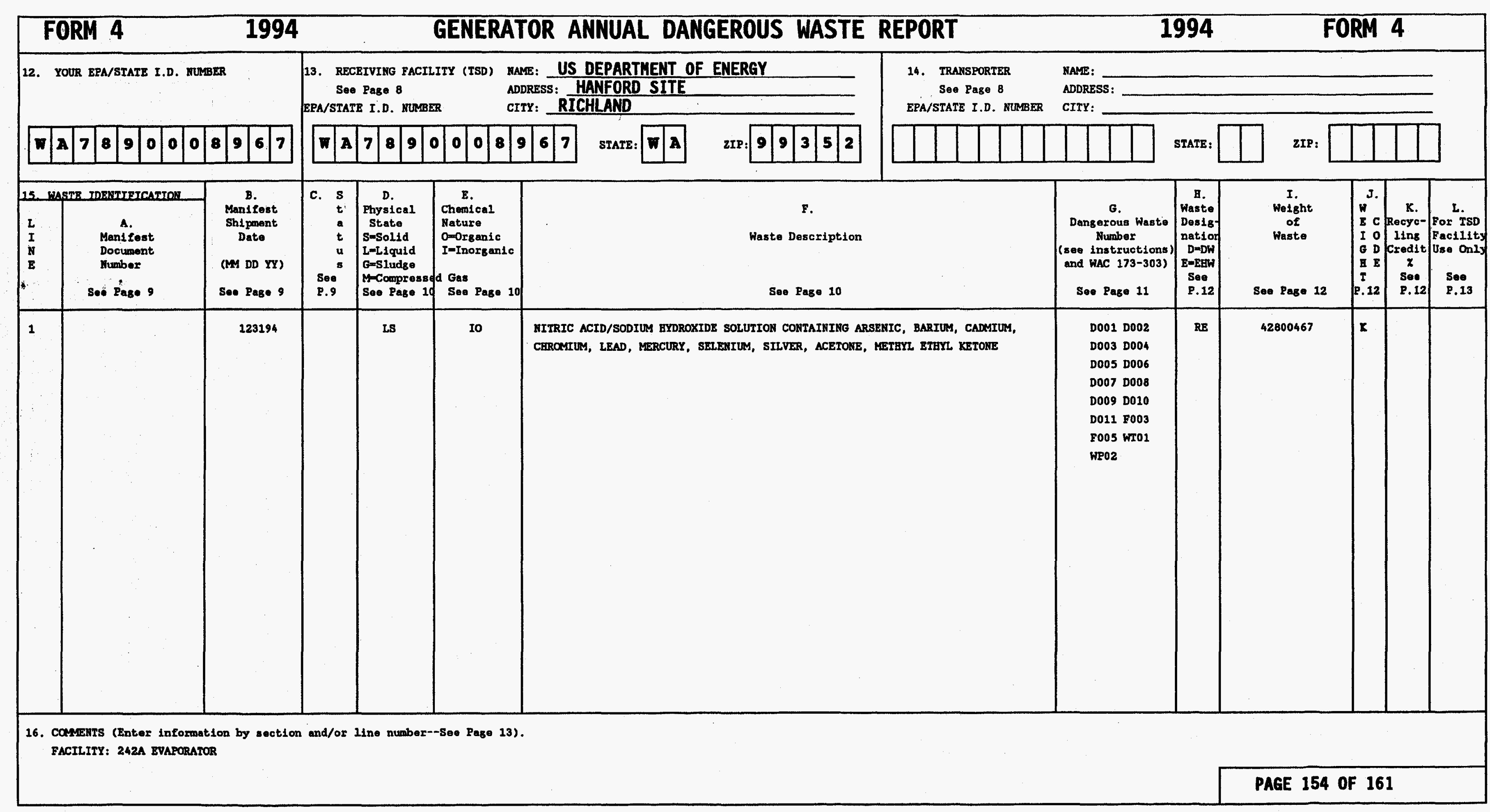




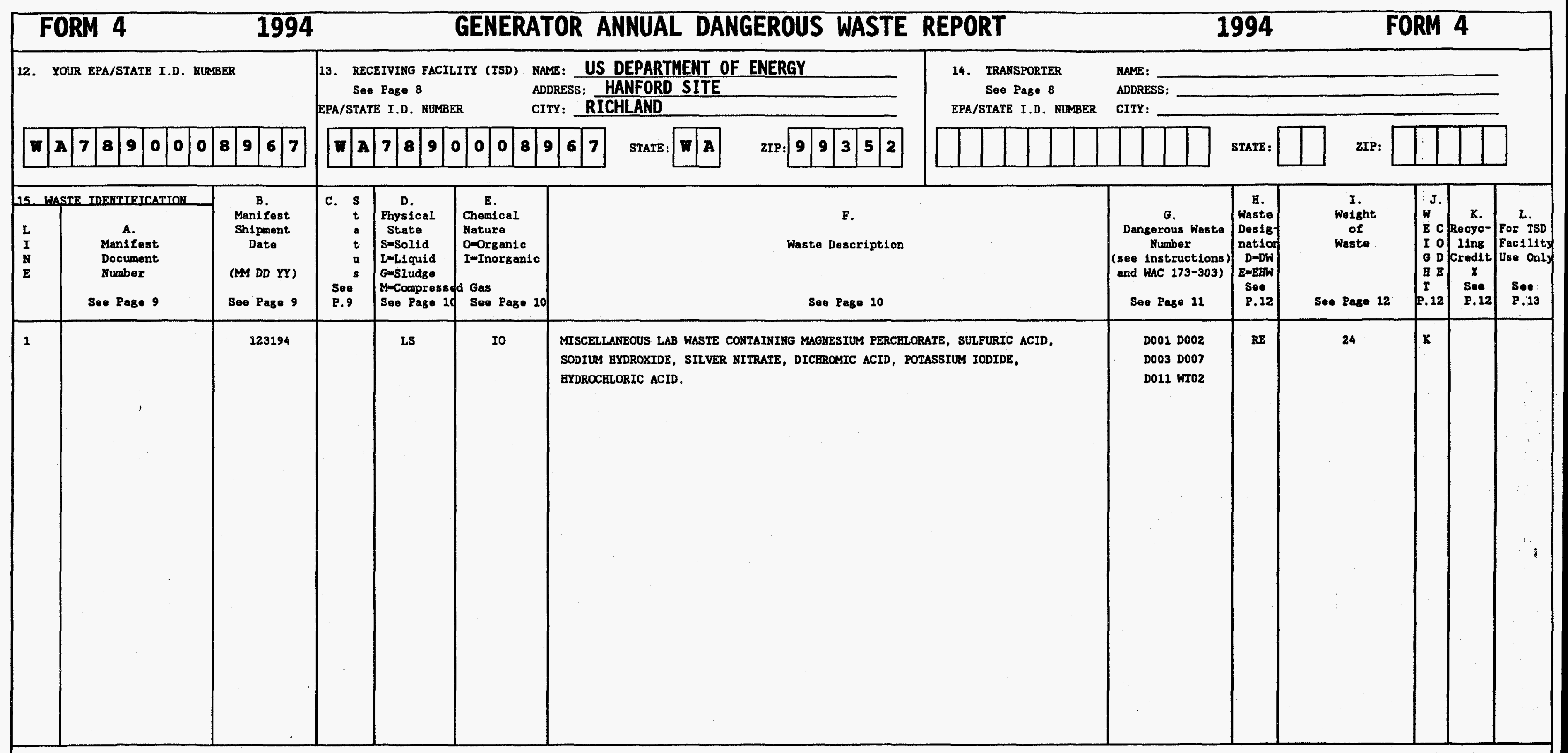

16. CongNIs (Enter Information by section and/or line number--See Page 13) FACILITY: 325 SAL TRMT UNIT

TRANSFERRED TO DOUBLE SHELL TANKS FOR STORAGE.

PAGE 155 OF 161 


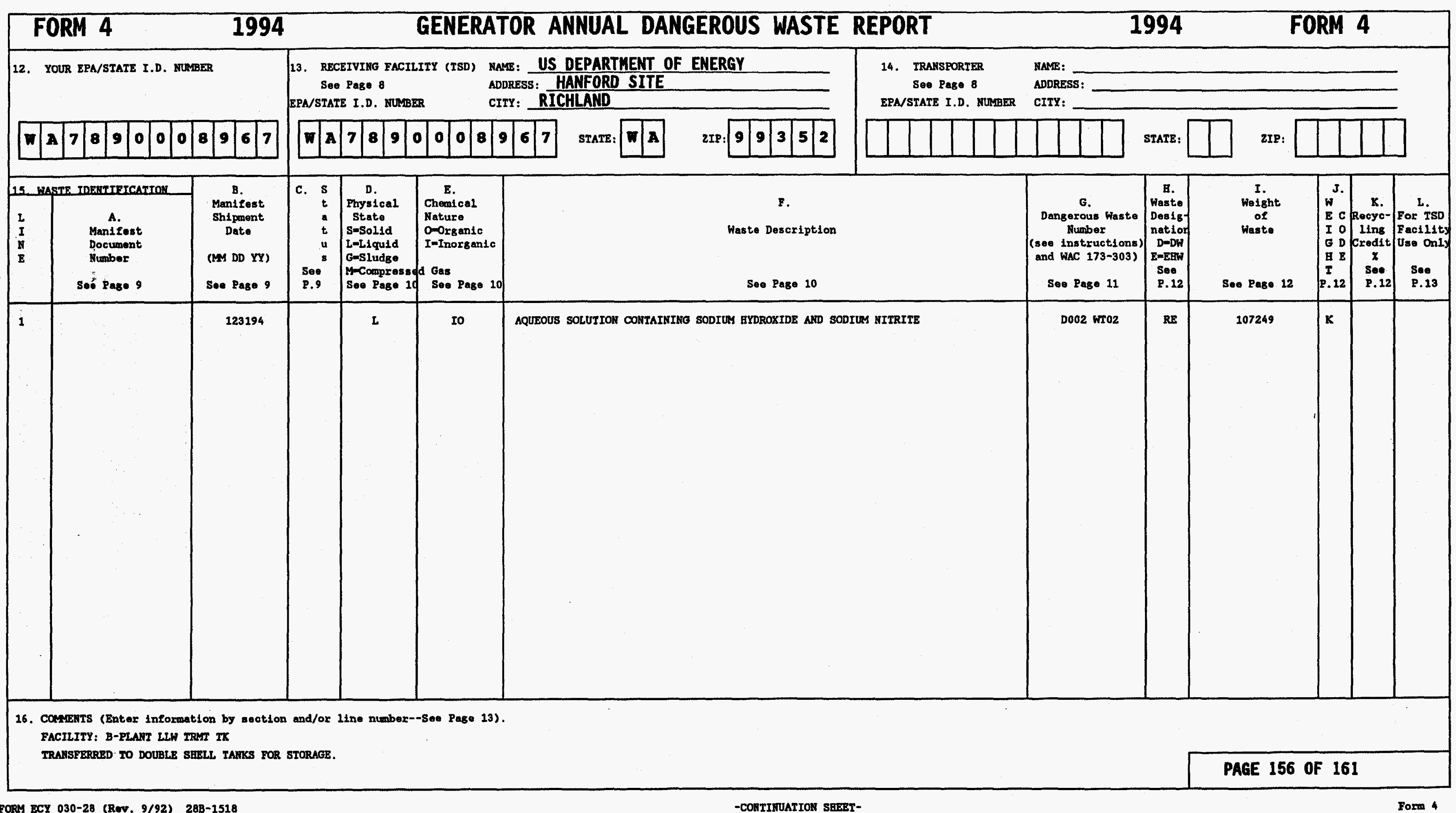




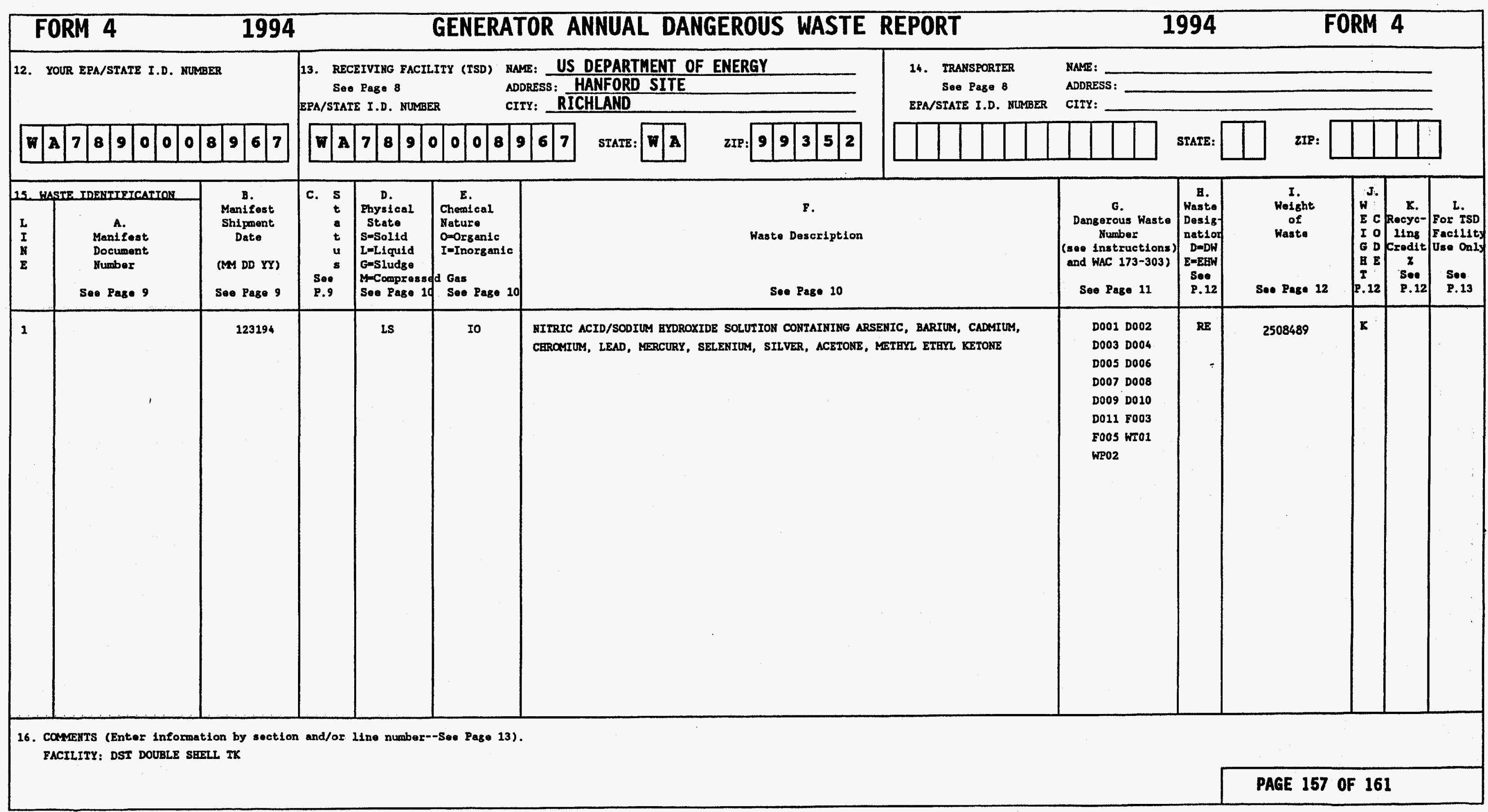




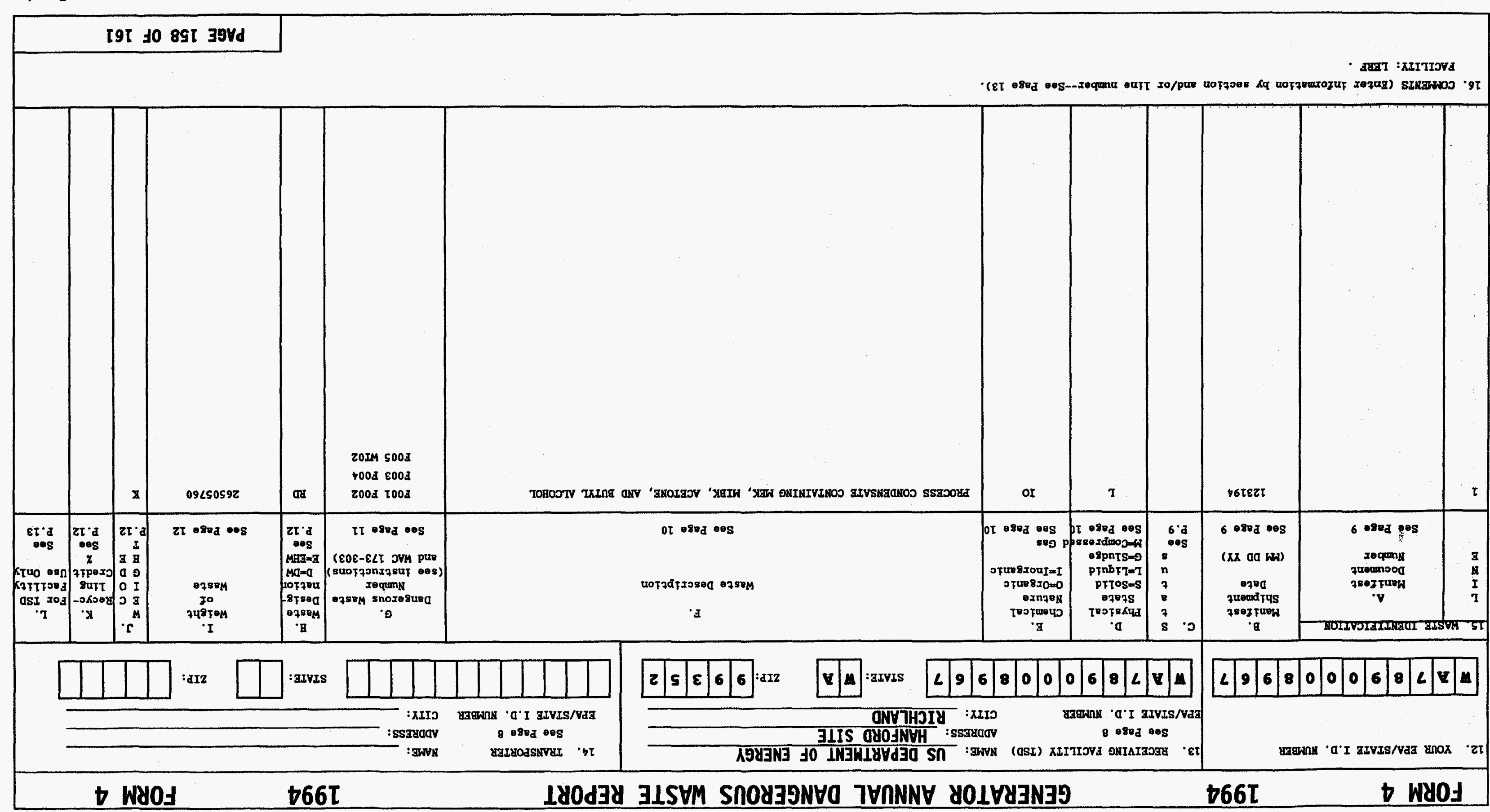




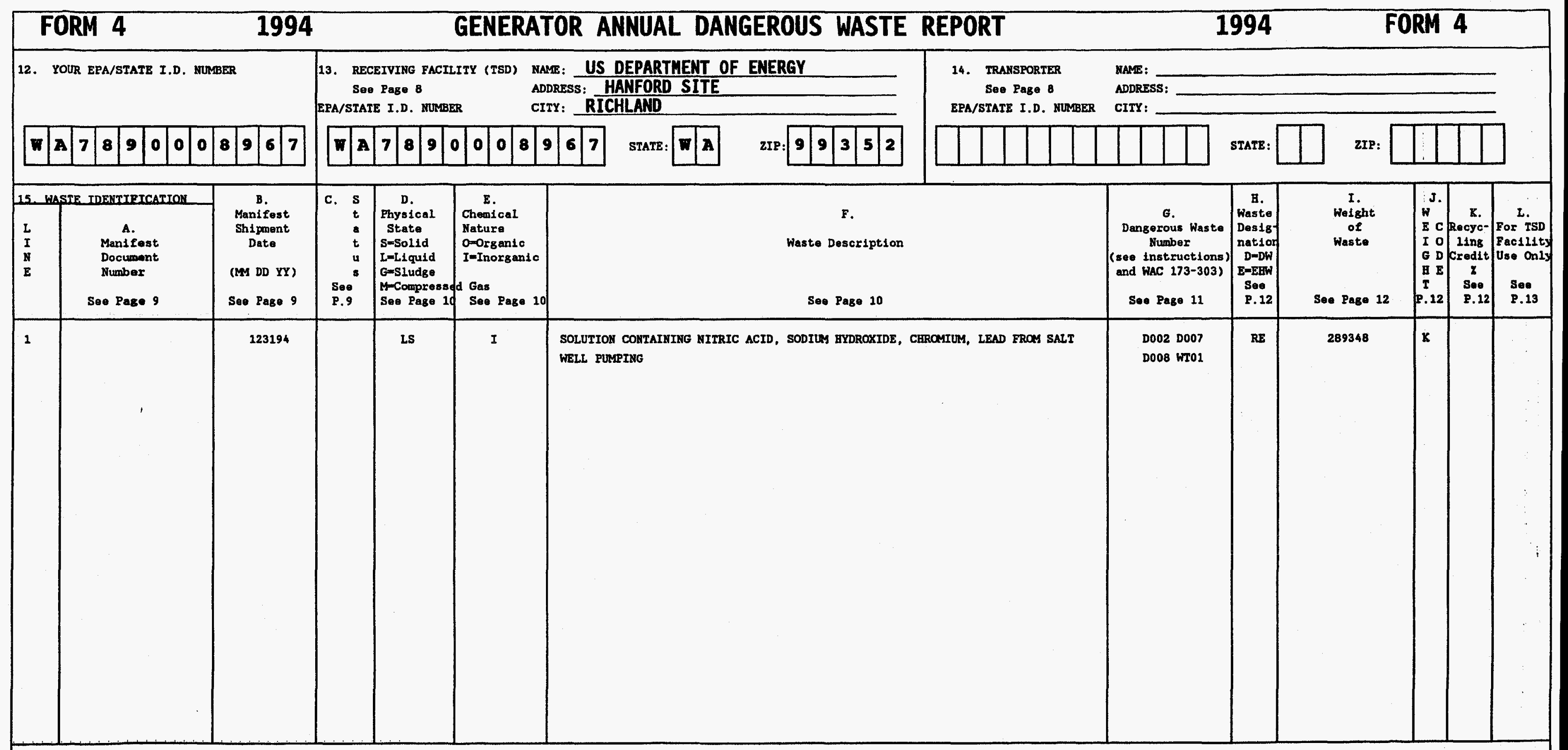

16. COMMENTS (Enter information by section and/or line number--See Page 13). FACILITY: SST SINGLE SHELL IK

PAGE 159 OF 161 


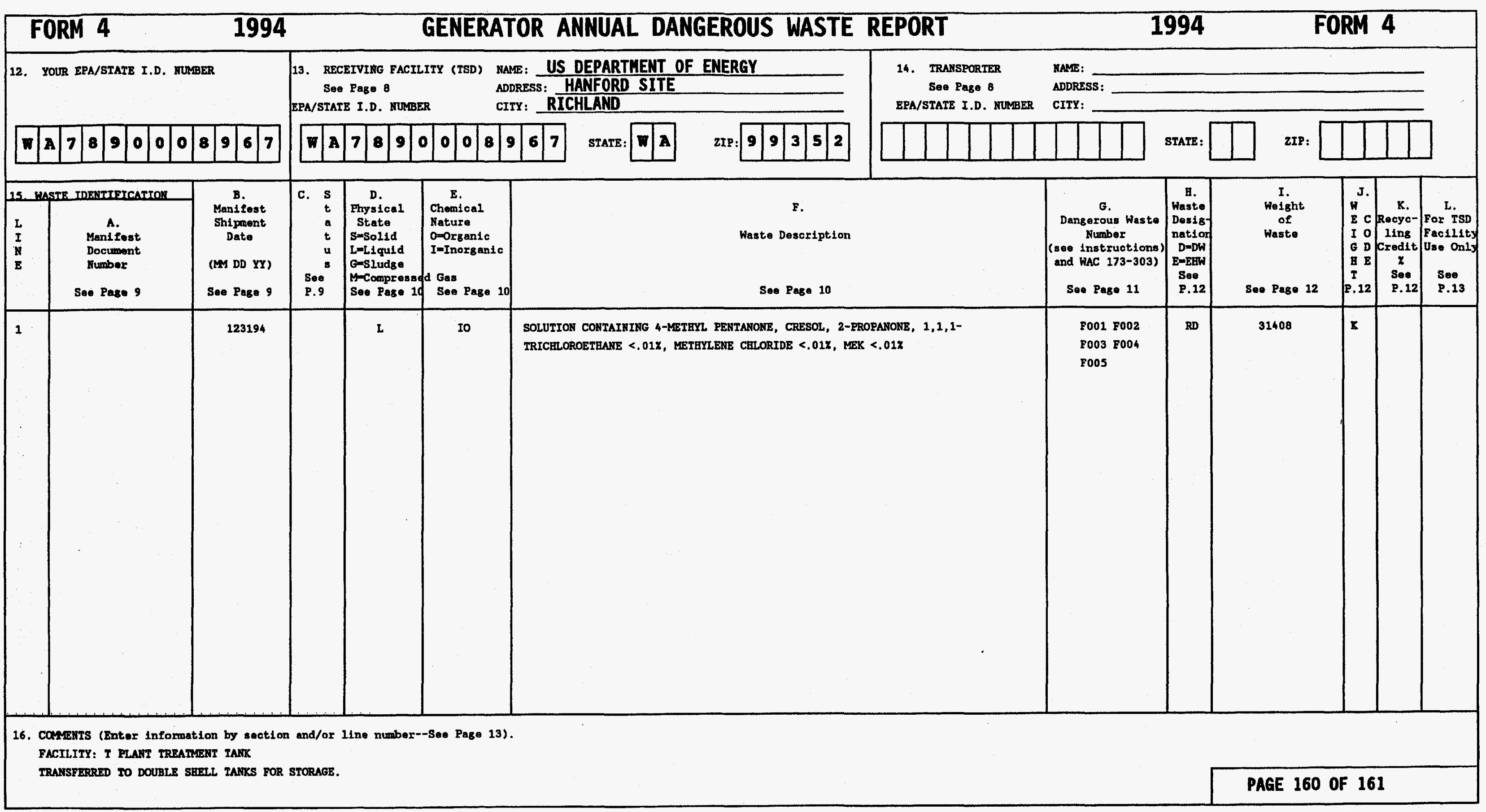




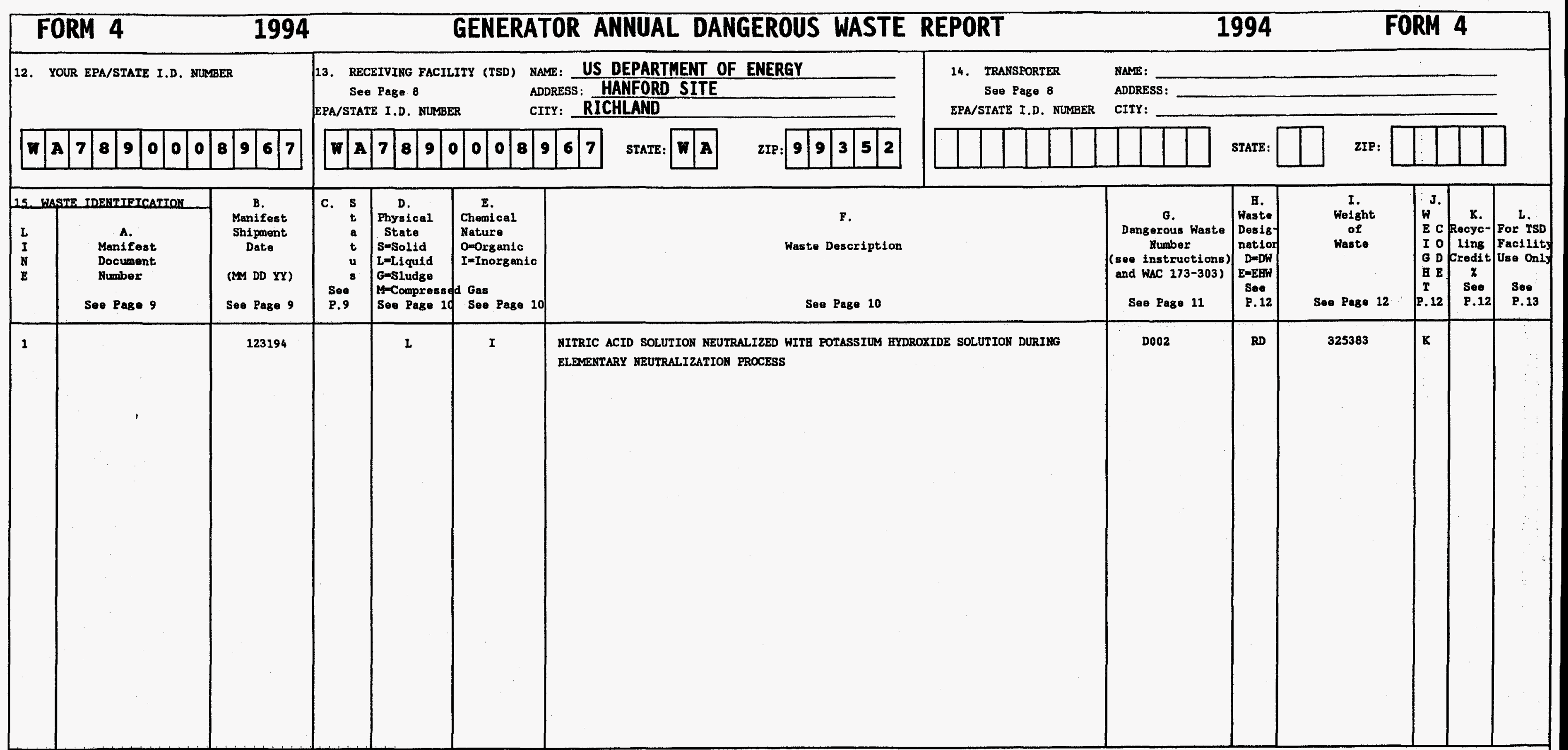

16. Coments (Entor Information by section and/or 1ine number--See Page 13) FACILITY: U03 C5 TANK

DISCEARGED UNDER DOE-RL/WDOE CONSENT ORDER DE 91NM-177. 


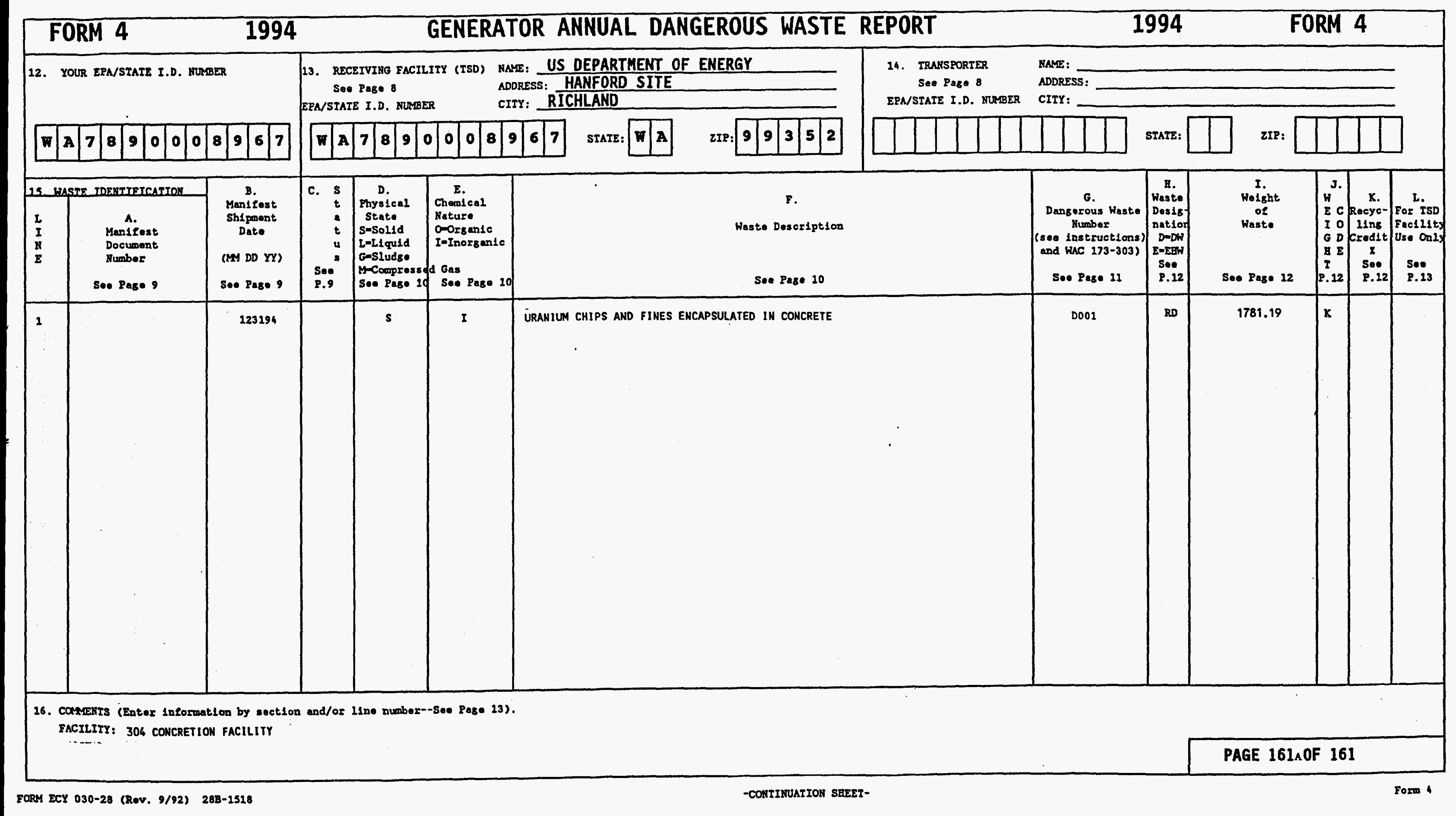

